\title{
Meaningful use of activity trackers in healthcare
}

Citation for published version (APA):

Ummels, D. (2021). Meaningful use of activity trackers in healthcare: Towards a personalised use in people with a chronic disease and older adults. [Doctoral Thesis, Maastricht University]. Maastricht University. https://doi.org/10.26481/dis.20211001du

Document status and date:

Published: 01/01/2021

DOI:

10.26481/dis.20211001du

Document Version:

Publisher's PDF, also known as Version of record

\section{Please check the document version of this publication:}

- A submitted manuscript is the version of the article upon submission and before peer-review. There can be important differences between the submitted version and the official published version of record.

People interested in the research are advised to contact the author for the final version of the publication, or visit the DOI to the publisher's website.

- The final author version and the galley proof are versions of the publication after peer review.

- The final published version features the final layout of the paper including the volume, issue and page numbers.

Link to publication

\footnotetext{
General rights rights.

- You may freely distribute the URL identifying the publication in the public portal. please follow below link for the End User Agreement:

www.umlib.nl/taverne-license

Take down policy

If you believe that this document breaches copyright please contact us at:

repository@maastrichtuniversity.nl

providing details and we will investigate your claim.
}

Copyright and moral rights for the publications made accessible in the public portal are retained by the authors and/or other copyright owners and it is a condition of accessing publications that users recognise and abide by the legal requirements associated with these

- Users may download and print one copy of any publication from the public portal for the purpose of private study or research.

- You may not further distribute the material or use it for any profit-making activity or commercial gain

If the publication is distributed under the terms of Article $25 \mathrm{fa}$ of the Dutch Copyright Act, indicated by the "Taverne" license above, 


\title{
Meaningful use of activity trackers in healthcare
}

\author{
Towards a personalised use in people
} with a chronic disease and older adults

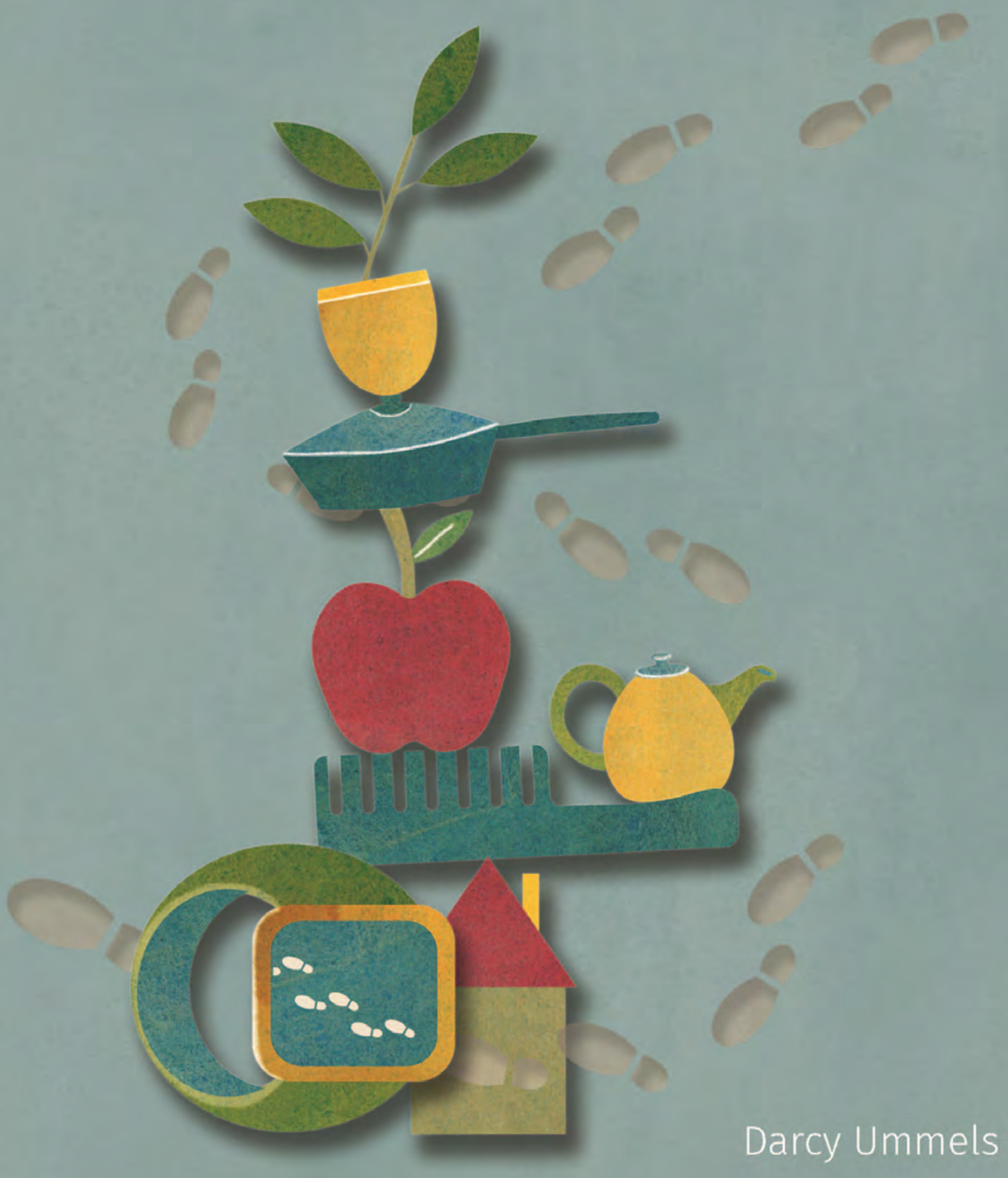


(C) Darcy Ummels, Maastricht 2021

For all articles published, the copyright has been transferred to the respective publisher. No part of this thesis may be reproduced in any form or by any means without written permission from the author or, when appropriate, from the publisher.

Cover: Evelien Jagtman

Lay-out: Tiny Wouters

Printed by: Ridderprint | www.ridderprint.nl

ISBN: 978-94-6416-651-4

The research in this dissertation was conducted at: The School for Public Health and Primary Care (CAPHRI), Department of Family Medicine, Maastricht University and The Research Centre for Autonomy and Participation for Persons with a Chronic Illness, Faculty of Health, Zuyd University of Applied Sciences.

The research in this dissertation was funded by Nationaal Regieorgaan Praktijkorgaan Onderzoek SIA (2014-01-54P and KIEM.VRIJ.04.059) and Brightlands Innovation Program LIME (Limburg Meet), a program that facilitates smarter measurement methods and more efficient data collection for better care and health, Province of Limburg, Zuyd University of applied sciences, Maastricht University.

Support for finalising this dissertation was kindly provided by ParaMedisch Centrum Zuid.

The printing of this thesis was financially supported by the Scientific College Physical Therapy (WCF) of the Royal Dutch Society for Physical Therapy (KNGF). 


\title{
Meaningful use of activity trackers in healthcare
}

\author{
Towards a personalised use in people with a \\ chronic disease and older adults
}

\author{
Proefschrift
}

ter verkrijging van de graad van doctor aan de Universiteit Maastricht, op gezag van de Rector Magnificus, Prof.dr. Rianne M. Letschert, volgens het besluit van het College van Decanen, in het openbaar te verdedigen op vrijdag 1 oktober 2021 om 12:00 uur

door

Darcy Ummels 


\section{Promotor}

Prof. dr. A.J.H.M. Beurskens

\section{Co-promotores}

Dr. E. Beekman

Dr. S.M. Braun

\section{Beoordelingscommissie}

Prof. dr. R.M.M. Crutzen (voorzitter)

Prof. dr. A.F. Lenssen

Prof. dr. C.A.W. Bolman, Open Universiteit

Dr. ir. M. Hettinga, Windesheim University of Applied Sciences

Dr. B.A.J. Berendsen 


\section{Contents}

$\begin{array}{lll}\text { Chapter } 1 \text { General introduction } & 7\end{array}$

Chapter 2 Counting Steps in Activities of Daily Living in People With a Chronic

Disease Using Nine Commercially Available Fitness Trackers:

Cross-Sectional Validity Study

JMIR Mhealth Uhealth, 2018; 2(6);e70

Chapter 3 Patients' experiences with commercially available activity trackers

embedded in physiotherapy treatment: a qualitative study

Disability and Rehabilitation, 2020; 42(23), 3284-3292

Chapter 4 Measure It Super Simple (MISS) activity tracker: (re)design of a user-friendly interface and evaluation of experiences in daily life Disability and Rehabilitation: Assistive technology, 2020; 24;1-11 online ahead of print

Chapter 5 The Validation of a Pocket Worn Activity Tracker for Step Count and Physical Behavior in Older Adults during Simulated Activities of Daily Living Gerontology and Geriatric medicine, 2020; 30-6;2333721420951732

Chapter 6 Using an activity tracker in healthcare: experiences of healthcare professionals and patients

Int J Environ Res Public Health. 2021 May; 18(10): 5147

Chapter 7 General discussion

Addendum English summary

Samenvatting

Impact paragraph

Dankwoord

About the author 



\section{Chapter 1}

General introduction 



\section{General introduction}

\section{Participation in daily life}

Engaging in daily life activities enhances an individual's wellbeing as well as increases their participation in society. ${ }^{1}$ One of the pre-conditions for this engagement in daily activities is sufficient physical capacity. Physical capacity refers to the ability to perform activities that require physical actions ${ }^{2,3}$ and consists of cardiovascular capacity, muscular capacity, and balance. ${ }^{4,5}$ Another pre-condition, physical activity is defined as any bodily movement produced by skeletal muscles that results in energy expenditure. ${ }^{2}$ Physical capacity and physical activity are strongly linked but not interchangeable. Sufficient physical activity does not guarantee sufficient physical activity and vice versa. ${ }^{6,7}$ When an individual lacks physical capacity or physical activity, their ability to participate in daily activities is reduced, which can lead to disablement (i.e., reduced participation in or complete withdrawal from society). ${ }^{8-10} \mathrm{~A}$ meta-analysis showed that increasing daily physical activity is not only the most effective method of preventing and slowing disablement ${ }^{11}$ but can also increase physical capacity. ${ }^{12-15}$ Sufficient physical activity is also associated with a reduced risk of chronic diseases such as psychiatric diseases (e.g., depression), neurological diseases (e.g., dementia), metabolic diseases (e.g., Diabetes Mellitus), cardiovascular diseases (e.g., coronary heart disease), pulmonary diseases (e.g., chronic obstructive pulmonary disease), musculoskeletal disorders (e.g., osteoporosis) and cancer. ${ }^{16-18}$

To determine whether an individual is sufficiently active, physical activity norms were introduced. In 2016, the recommendation for adults in the Netherlands was to be moderately physically active for 30 minutes 5 days per week. ${ }^{19}$ Another guideline-to achieve 10,000 steps per day-gained popularity in the media and may serve as an alternative physical activity norm. ${ }^{20}$ However, recent studies have suggested that the 10,000 steps per day guideline may not be suitable for every population. For instance, it may be inappropriate for older adults or people with a chronic disease. ${ }^{21}$

\section{Measuring physical activity: why and how}

Physical activity can be measured to gain insight into an individual's physical activity level as well as the types, amount, and distribution of physical activity they perform. A study showed that individuals experience difficulties estimating whether they meet the amount of physical activity recommended by the aforementioned guidelines; about half of the inactive participants in this study overestimated their physical activity level and believed they were sufficiently active. ${ }^{22}$ In addition to generating insights, measuring physical activity can also produce objective feedback that can be used to change one's 
physical activity behaviours (e.g., increase one's physical activity level). Physical activity can be measured by numerous variables, such as step count, active minutes, heart rate, walked distance, type of activity (e.g., sitting), and burned calories. Most variables of physical activity can be measured in either an objective or subjective way. Subjective measures include self-report questionnaires and diaries ${ }^{23}$; the former is currently the most used method to measure physical activity. ${ }^{24}$ Frequently used questionnaires are the Global Physical Activity Questionnaire (GPAQ), ${ }^{25}$ International Physical Activity Questionnaire (IPAQ), ${ }^{26}$ Physical Activity Scale for the Elderly (PASE), ${ }^{27}$ and the Short Questionnaire to Assess Health-enhancing physical activity (SQUASH). ${ }^{28}$ In a review, 85 self-reported questionnaires for adults were presented. ${ }^{29}$ The validity and reliability properties of the questionnaires were assessed in 76 different questionnaires, from which only a few had sufficient validity and reliability. ${ }^{29}$ This review stated that there is a lack of standardisation among questionnaires and concluded that no questionnaires could be (strongly) recommended above others. Overall, several studies showed that using questionnaires and diaries to self-report physical activity tend to result in over- or underestimations of actual physical activity. ${ }^{30}$ In addition to their lack of good clinometric properties, questionnaires, and diaries also lack feasibility; they are timeconsuming and contingent on the participant's memory. ${ }^{31}$

Objective measurements of physical activity can be performed with doubly labelled water or indirect calorimetry. ${ }^{23}$ Contrary to questionnaires and diaries, these measurement tools have good validity and reliability and do not rely on the participant's memory. ${ }^{32,33}$ However, these methods also lack feasibility for daily practice; they are not accessible to the average individual, cost more and require more expertise than questionnaires and diaries, and are equally time-consuming. ${ }^{34}$ Another method of objectively measuring physical activity involves the use of activity trackers, which are potentially objective, easy to use, and commercially available.

\section{Activity trackers}

Activity trackers are primarily used in research and sports. Recently, however, activity trackers have become available for commercial use and are gaining popularity. A distinction can be made between consumer-grade activity trackers (e.g., Fitbit) and nonconsumer grade activity trackers (e.g., activPAL). Non-consumer-grade activity trackers are predominantly used in research settings and often require special software or additional licenses. Meanwhile, there is a plethora of consumer-grade activity trackers from which to choose. In 2016, the top five brands combined sold 102.4 million activity trackers worldwide. ${ }^{35}$ In 2016, one out of three Dutch citizens was using either an app, smartwatch, or activity tracker. ${ }^{36}$ Depending on the type of activity tracker, it can 
continuously and concurrently measure multiple variables, such as walked distance, heart rate, number of stairs climbed, and time spent in a given position (e.g., sitting). Activity trackers can be divided into three basic categories: pedometers, accelerometers, and apps. Pedometers generally only measure the number of steps, whereas accelerometers can measure more variables such as step count or heart rate. Both pedometers and accelerometers can typically be worn on several places such as the wrist, trouser pocket, and brassiere. Most smartphones and smartwatches include builtin accelerometers. Apps on smartphones and smartwatches can then use this accelerometer and thus require no additional device (e.g., an accelerometer on the wrist), contrary to pedometers and accelerometers.

\section{Potential use of activity trackers in healthcare}

Insight into a patient's physical activity level aids daily clinical practice for both healthcare professionals and patients, since these data can be used for diagnostic, prognostic, and evaluative purposes. ${ }^{37}$ Clinical practice guidelines advise healthcare professionals, such as physiotherapists, to measure the physical activity level of their patients, most of whom are older adults or people with a chronic disease, with questionnaires and diaries. ${ }^{38-43}$ Activity trackers can serve as an alternative or supplement to such measurement tools. In particular, consumer-grade activity trackers have potential, since they tend to be easy-to-use for both healthcare professionals and patients and, unlike non-consumer-grade activity trackers, do not require additional licenses or software costs. Consumer-grade activity trackers can offer objective insight into patients' physical activity level and could potentially support the clinical reasoning of healthcare professionals. Next to that, studies have shown that the use of activity trackers can enhance patients' self-management and self-efficacy. ${ }^{44,45}$ Self-monitoring could also lead to improved patient's coping, realistic goal setting, and improved quality of life. ${ }^{46}$ Furthermore, using activity trackers in combination with regular exercise or lifestyle guidance has been shown to improve the physical activity level of patients with a chronic disease and older adults. ${ }^{47-50}$ Healthcare professionals, such as physiotherapists, $^{51}$ nurses, $^{52}$ nutritionists, $^{53}$ and general practitioners, ${ }^{54}$ are trained to provide lifestyle guidance. Hence, there are abundant advantages to integrating activity trackers into healthcare, yet they are seldom used.

\section{Validity of activity trackers in older adults and people with a chronic disease}

Several validity and reliability studies of consumer-grade activity trackers have been

performed in healthy adults. ${ }^{55-57}$ A systematic review showed that they offer good validity for step count, a lower validity for energy and sleep, and a high reliability for step 
count, distance, energy expenditure, and sleep. ${ }^{57}$ However, these results cannot be generalised to older adults and people with a chronic disease because such groups often have altered walking patterns-due to impaired ambulatory abilities-as well as lower walking speeds (e.g., shuffling) which are variables used by the algorithm. ${ }^{58-62}$ Furthermore, the most important source of physical activity for older adults and people with a chronic disease are household activities, gardening, and walking, ${ }^{19}$ and low walking speed often accompany these activities. It is known that low walking speed $(<0.8 \mathrm{~m} / \mathrm{s})$ decreases the validity of activity trackers. ${ }^{63-67}$ Only few studies have examined the validity of activity trackers among older adults and people with a chronic disease. ${ }^{68}$ However, the results of these studies cannot be generalized, as they did not consider the activities of daily living (e.g., free-living protocol), did not use a gold standard, or only used non-commercially available activity trackers. ${ }^{68}$

\section{Feasibility of activity trackers for older adults and people with a chronic disease in} healthcare

Another important property concerns the feasibility of activity trackers. Feasibility is a broad term that includes experiences, acceptability, and usefulness. Studies in healthy adults, ${ }^{45,69-73}$ older adults, ${ }^{74-76}$, and people with a chronic disease ${ }^{77,78}$ showed that, in general, these target populations are open to using consumer-grade activity trackers. However, older adults and people with a chronic disease indicate that their limited technical skills discourage them from using such trackers. Moreover, no studies have investigated the feasibility of activity trackers or analysed how to embed them in daily clinical practice. The possibility of using activity trackers in healthcare is new, and no guidelines or studies are available to support healthcare professionals in selecting and using activity trackers in their daily clinical practice.

\section{Aim and outline of this dissertation}

There is little to no evidence regarding the validity, feasibility, and use of activity trackers in the healthcare of older adults and people with a chronic disease. This may explain why activity trackers have not been implemented in healthcare, despite the fact that they could potentially be of significant value. Therefore, the main aim of this dissertation is to increase the knowledge about the meaningful use of activity trackers in healthcare for (older) adults with chronic diseases and for older adults with or without chronic diseases. 
The word 'meaningful' refers to our intention that the use of an activity tracker should be personalised and incorporated into daily life and healthcare in a manner that the data support both the patient and healthcare professional. It should provide insight into the patient's activity levels, aid decisions, and support the formulation of recommendations for changing the patient's behavioural habits towards a healthier lifestyle.

This dissertation has three phases, in which five studies were performed (Figure 1.1).

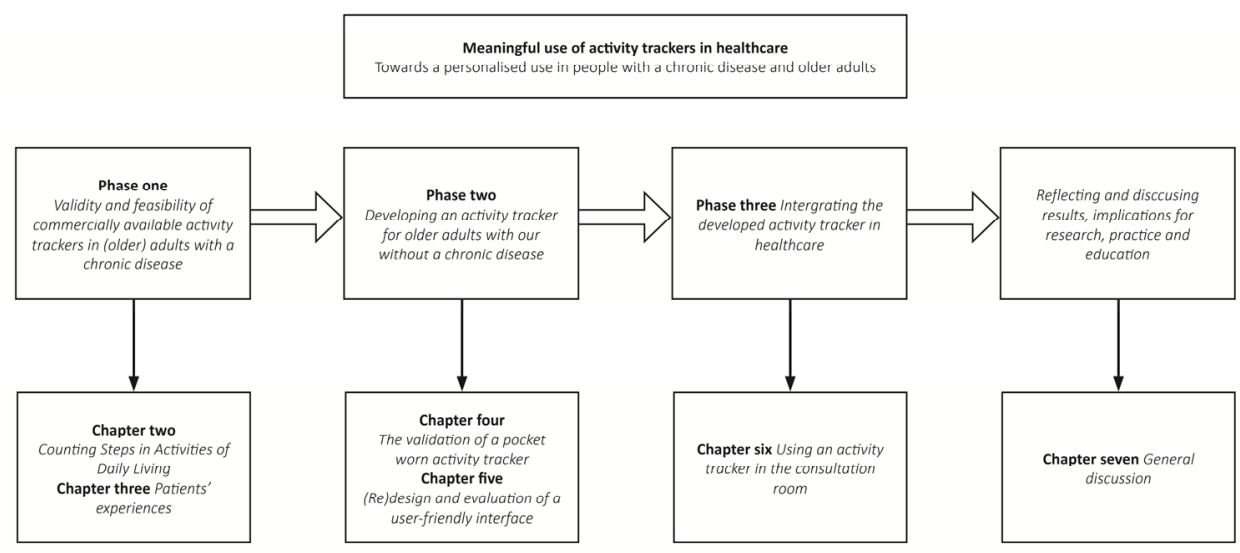

Figure 1.1 Outline of the dissertation.

Phase One (Chapters Two and Three) studied the validity and feasibility of commercially available activity trackers in (older) adults who have chronic diseases and are receiving physiotherapy. Phase One addressed the following two research questions:

- What is the validity of commercially available activity trackers during activities of daily living in (older) adults with a chronic disease?

- How do (older) adults with a chronic disease experience the implementation of an activity tracker into their physiotherapy treatment?

With the results of Phase One, a 'new' activity tracker was developed in Phase Two (Chapters Four and Five), by (re)designing the user interface of an existing activity tracker and developing an algorithm targeted at older adults with or without a chronic disease. The feasibility of the developed activity tracker was tested by older adults with or without a chronic disease. Phase Two addressed the following three research questions:

- How can a non-commercial activity tracker be developed into a feasible measurement tool for older adults with or without a chronic disease? 
- What is the validity of the developed activity tracker in older adults with or without a chronic disease during activities of daily living?

- How do older adults with or without a chronic disease experience the adapted activity tracker during their daily lives?

Using the knowledge generated during Phase One and Phase Two, the developed activity tracker was integrated into the workflow of healthcare professionals in Phase Three (Chapter 6). Phase Three addressed the following research question:

- How can an activity tracker be integrated into the daily clinical practice of healthcare professionals?

Chapter Two analyses the validity of the step count in nine commercially available activity trackers used during the daily activities of (older) adults with a chronic disease. Chapter Three examines how (older) adults with a chronic disease experience the incorporation of activity trackers into their regular physiotherapy sessions. Chapter Four assesses the validity of an adapted algorithm used by older adults with or without a chronic disease during activities of daily living. Chapter Five describes the user centred development of an activity tracker for (older) adults with or without a chronic disease and the feasibility test of the developed activity tracker. In Chapter Six, the developed activity tracker will be integrated into the daily clinical practice of healthcare professionals. Chapter Seven summarises the primary findings of this dissertation, discusses the methodology, and outlines implications for future research, practice, and education.

This dissertation was part of the Brightlands Innovation Programme Limburg Meet (LIME), ${ }^{79}$ a programme that facilitates the development of smarter measurement methods and more efficient data collection for better care and health. LIME also facilitates and supervises projects that develop innovative measuring products or processes. Within the LIME program, several themes were distinguished. One of these themes is 'personalised wearables,' of which this PhD project was a part. The goal of this theme was to help new wearables fulfil their potential, produce more personalised care, and achieve a higher level of self-reliance for clients and citizens by playing an active role in the further development and implementation of wearables in healthcare. 


\section{References}

1. Kahneman D, Diener E, Schwarz N. Well-Being: Foundations of Hedonic Psychology. $1^{\text {st }}$ edition. New York: Russell Sage Foundation; 1999. ISBN: 0871544237

2. Caspersen CJ, Powell KE, Christenson GM. Physical activity, exercise, and physical fitness: definitions and distinctions for health-related research. Public Health Rep 1985; 100(2): 126-31.

3. Puente-Maestu L, Palange $P$, Casaburi R, et al. Use of exercise testing in the evaluation of interventional efficacy: an official ERS statement. The European respiratory journal 2016; 47(2): 429-60.

4. Morree JJ JM, van der Poel G. Inspanningsfysiologie, oefentherapie en training. $2^{\text {nd }}$ eidtion, Houten: Bohn Stafleu van Loghum; 2006. ISBN: 9789031387328.

5. Mesquita R, Janssen DJ, Wouters EF, Schols JM, Pitta F, Spruit MA. Within-day test-retest reliability of the Timed Up \& Go test in patients with advanced chronic organ failure. Arch Phys Med Rehabil 2013; 94(11): 2131-8.

6. Bootsma-van der Wiel A, Gussekloo J, de Craen AJ, et al. Disability in the oldest old: "can do" or "do do"? Journal of the American Geriatrics Society 2001; 49(7): 909-14.

7. van Lummel RC, Walgaard S, Pijnappels M, et al. Physical Performance and Physical Activity in Older Adults: Associated but Separate Domains of Physical Function in Old Age. PloS one 2015; 10(12): e0144048.

8. Verbrugge LM, Jette AM. The disablement process. Social Science \& Medicine 1994; 38(1): 1-14.

9. Vermeulen J, Neyens JC, van Rossum E, Spreeuwenberg MD, de Witte LP. Predicting ADL disability in community-dwelling elderly people using physical frailty indicators: a systematic review. BMC Geriatr 2011; 11: 33.

10. Stuck AE, Walthert JM, Nikolaus T, Büla CJ, Hohmann C, Beck JC. Risk factors for functional status decline in community-living elderly people: a systematic literature review. Social Science \& Medicine 1999; 48(4): 445-69.

11. Tak E, Kuiper R, Chorus A, Hopman-Rock M. Prevention of onset and progression of basic ADL disability by physical activity in community dwelling older adults: a meta-analysis. Ageing Res Rev 2013; 12(1): 329-38.

12. Wahid A, Manek N, Nichols M, et al. Quantifying the Association Between Physical Activity and Cardiovascular Disease and Diabetes: A Systematic Review and Meta-Analysis. J Am Heart Assoc 2016; 5(9).

13. Nocon M, Hiemann T, Muller-Riemenschneider F, Thalau F, Roll S, Willich SN. Association of physical activity with all-cause and cardiovascular mortality: a systematic review and meta-analysis. Eur J Cardiovasc Prev Rehabil 2008; 15(3): 239-46.

14. Leblanc A, Pescatello LS, Taylor BA, et al. Relationships between physical activity and muscular strength among healthy adults across the lifespan. Springerplus 2015; 4: 557.

15. Ferreira ML, Sherrington $C$, Smith K, et al. Physical activity improves strength, balance and endurance in adults aged 40-65 years: a systematic review. Journal of Physiotherapy 2012; 58(3): 145-56.

16. Pedersen BK, Saltin B. Exercise as medicine - evidence for prescribing exercise as therapy in 26 different chronic diseases. Scandinavian journal of medicine \& science in sports 2015; 25 Suppl 3: 1-72.

17. Booth FW, Roberts CK, Laye MJ. Lack of exercise is a major cause of chronic diseases. Compr Physiol 2012; 2(2): 1143-211.

18. Warburton DER, Bredin SSD. Health benefits of physical activity: a systematic review of current systematic reviews. Curr Opin Cardiol 2017; 32(5): 541-56.

19. Hildebrandt VH, Bernaards CM, Hofstetter H. Trendrapport Bewegen en Gezondheid 2000/2014. 1ste edition. Ridderkerk: TNO: Hollandridderkerk; 2014.

20. Choi BC, Pak AW, Choi JC, Choi EC. Daily step goal of 10,000 steps: a literature review. Clin Invest Med 2007; 30(3): E146-51.

21. Tudor-Locke $C$, Craig CL, Aoyagi $Y$, et al. How many steps/day are enough? For older adults and special populations. Int J Behav Nutr Phys Act 2011; 8: 80.

22. Godino JG, Watkinson C, Corder K, Sutton S, Griffin SJ, van Sluijs EM. Awareness of physical activity in healthy middle-aged adults: a cross-sectional study of associations with sociodemographic, biological, behavioural, and psychological factors. BMC Public Health 2014; 14: 421. 
23. Bakker EA, Eijsvogels TMH, de Vegt F, Busser GSF, M.T.E. H, Verbeek ALM. Patiënten in beweging. Gevalideerde methoden om lichamelijke activiteit te kwantificeren. Nederlands Tljdschrift voor Geneeskunde 2015; 159.

24. Sylvia LG, Bernstein EE, Hubbard JL, Keating L, Anderson EJ. Practical guide to measuring physical activity. J Acad Nutr Diet 2014; 114(2): 199-208.

25. Armstrong T, Bull F. Development of the World Health Organization Global Physical Activity Questionnaire (GPAQ). Journal of Public Health 2006; 14(2): 66-70.

26. Craig $\mathrm{CL}$, Marshall $\mathrm{AL}$, Sjostrom $\mathrm{M}$, et al. International physical activity questionnaire: 12-country reliability and validity. Med Sci Sports Exerc 2003; 35(8): 1381-95.

27. Washburn R, Smith K, Jette A, CA. J. The Physical Activity Scale for the Elderly (PASE): development and evaluation. J Clin Epidemiol 1993; 46(2): 153-62.

28. Wendel-Vos GC, Schuit AJ, Saris WH, Kromhout D. Reproducibility and relative validity of the short questionnaire to assess health-enhancing physical activity. J Clin Epidemiol 2003; 56(12): 1163-9.

29. van Poppel MN, Chinapaw MJ, Mokkink LB, van Mechelen W, Terwee CB. Physical activity questionnaires for adults: a systematic review of measurement properties. Sports medicine (Auckland, NZ) 2010; 40(7): 565-600.

30. Prince SA, Adamo KB, Hamel ME, Hardt J, Connor Gorber S, Tremblay M. A comparison of direct versus self-report measures for assessing physical activity in adults: a systematic review. Int J Behav Nutr Phys Act 2008; 5: 56.

31. Ainsworth B, Cahalin L, Buman M, Ross R. The current state of physical activity assessment tools. Progress in cardiovascular diseases 2015; 57(4): 387-95.

32. Buchowski MS. Doubly labeled water is a validated and verified reference standard in nutrition research. J Nutr 2014; 144(5): 573-4.

33. Haugen HA, Chan LN, Li F. Indirect calorimetry: a practical guide for clinicians. Nutr Clin Pract 2007; 22(4): 377-88.

34. Carlson-Newberry S, Costello R. Emerging Technologies for Nutrition Research: Potential for Assessing Military Performance Capability. $1^{\text {st }}$ edition. Washington (DC): National Academies Press (US); 1997. ISBN: 0309057973

35. International Data Coroporation. Wearables Aren't Dead, They're Just Shifting Focus as the Market Grows $16.9 \%$ in the Fourth Quarter, According to IDC. URL: https://www.idc.com/getdoc.jsp?containerld= prUS42342317 (accessed: 2017-11-17).

36. Renders C, Boots T, Visser K, Roozen B. Smart Health Monitor: Onderzoek naar gebruik van apps, wearables en meters. 1st edition. 's-Hertogenbosch: Multiscope; 2016.

37. Swinkels R, Meerhoff GA, Beekman E, Beurskens A. Raamwerk Klinimetrie voor evidence based products. URL: https://www.kngf.nl/binaries/content/assets/kngf/onbeveiligd/vak-en-kwaliteit/kwaliteit/klinimetrie/ raamwerk-klinimetrie-november-2016-def.pdf (accessed: 2021-03-08)

38. Köke AJA, van den Ende CHM, Jansen MJ, Steultjens MPM, Veenhof C. KNGF Standaard Beweeginterventie $\quad$ artrose. URL: https://www.fysionetevidencebased.nl/images/pdfs/beweeginterventies/standaard_bi_artrose_2011.pdf (accessed 2017-0707).

39. Köke AJA, Hilberdink S, Hilberdink WKHA, et al. KNGF-standaard Beweeginterventie chronische pijn. URL: https://www.fysionet

evidencebased.nl/images/pdfs/beweeginterventies/standaard_bi_chronische_pijn.pdf (accessed: 201707-07).

40. Praet SFE, van Uden C, Hartgens F, Savelberg HHCM, Toereppel K, de Bie RA. KNGF-standaard Beweeginterventie diabetes mellitus type 2. URL: https://www.fysionet-evidencebased.nl/images/ pdfs/beweeginterventies/standaard_bi_dm2_2009.pdf (accessed: 2017-07-07).

41. Stuiver MM, Wittink HM, Velthuis MJ, Kool N, Jongert WAM. KNGF-standaard Beweeginterventie oncologie. URL: https://www.fysionet-evidencebased.nl/images/pdfs/beweeginterventies/standaard_ bi_oncologie_2011.pdf (accessed: 2017-07-07).

42. Troosters T, Jongert MWA, de Bie RA, Toereppel K, de Gruijter EEMH. KNGF Standaard Beweeginterventie chronisch obstructieve longziekten. URL: https://www.fysionet-evidencebased.nl/images/pdfs/ beweeginterventies/standaard_bi_copd_2009.pdf (accessed: 2017-07-07). 
43. Verhagen SJM, Jongert MWA, Koers H, Toereppel K, Walhout $R$, Staal JB. KNGF-standaard Beweeginterventie coronaire hartziekten. URL: https://www.fysionet-evidencebased.nl/images/ pdfs/beweeginterventies/standaard_bi_corn_hartziekten_2009_update3.pdf (accessed: 2017-07-07).

44. Chiauzzi E, Rodarte C, DasMahapatra P. Patient-centered activity monitoring in the self-management of chronic health conditions. BMC medicine 2015; 13: 77.

45. Karapanos E, Gouveia R, Hassenzahl M, Forlizzi J. Wellbeing in the Making: Peoples' Experiences with Wearable Activity Trackers. Psychology of well-being 2016; 6: 4.

46. Richard AA, Shea K. Delineation of self-care and associated concepts. J Nurs Scholarsh 2011; 43(3): 25564.

47. Funk M, Taylor EL. Pedometer-based walking interventions for free-living adults with type 2 diabetes: a systematic review. Current diabetes reviews 2013; 9(6): 462-71.

48. Kawagoshi A, Kiyokawa N, Sugawara K, et al. Effects of low-intensity exercise and home-based pulmonary rehabilitation with pedometer feedback on physical activity in elderly patients with chronic obstructive pulmonary disease. Respiratory medicine 2015; 109(3): 364-71.

49. Mendoza L, Horta P, Espinoza J, et al. Pedometers to enhance physical activity in COPD: a randomised controlled trial. The European respiratory journal 2015; 45(2): 347-54.

50. King AC, Hekler EB, Grieco LA, et al. Effects of Three Motivationally Targeted Mobile Device Applications on Initial Physical Activity and Sedentary Behavior Change in Midlife and Older Adults: A Randomized Trial. PloS one 2016; 11(6): e0156370.

51. Crajé MC, Hodselmans AP, van Ittersum MW, van Heeringen-de Groot D, Verhoef J, van der Schans CP. Introduction Royal Dutch Society for Physical Therapy physical activity intervention. URL: https://www.fysionet-evidencebased.nl/images/pdfs/beweeginterventies/inleiding_bij_de_kngfstandaarden_beweeginterventies.pdf (accessed: 2017-07-07).

52. Schuurmans M, Lambregts J, Grotendorst A. Beroepsprofiel verpleegkundige. URL: http://www.invoorzorg.nl/docs/ivz/informatiecentrum/professionals/23203\%20Beroepsprofiel\%20verple egkundige.pdf (accessed 2021-03-08).

53. Nederlandse Vereniging van Dietisten. Beroepsprofiel diëtist URI:. https://www.nvdietist.nl/images/ Wetgeving/Beroepsprofiel_2013.pdf (accessed 2021-03-08).

54. van de Vijver P, van der Post K, Rosmalen K, et al. Competentieprofiel van de huisarts. URL: https://www.huisartsopleiding.nl/images/opleiding/Competentieprofiel_van_de_huisarts_2016.pdf (accessed 2021-03-08).

55. Kooiman TJ, Dontje ML, Sprenger SR, Krijnen WP, van der Schans CP, de Groot M. Reliability and validity of ten consumer activity trackers. BMC Sports Sci Med Rehabil 2015; 7: 24.

56. Ferguson $\mathrm{T}$, Rowlands $\mathrm{AV}$, Olds $\mathrm{T}$, Maher $\mathrm{C}$. The validity of consumer-level, activity monitors in healthy adults worn in free-living conditions: a cross-sectional study. Int J Behav Nutr Phys Act 2015; 12: 42.

57. Evenson KR, Goto MM, Furberg RD. Systematic review of the validity and reliability of consumer-wearable activity trackers. Int J Behav Nutr Phys Act 2015; 12: 159.

58. Lahousse L, Verlinden VJ, van der Geest JN, et al. Gait patterns in COPD: the Rotterdam Study. The European respiratory journal 2015; 46(1): 88-95.

59. Mueller MJ, Minor SD, Sahrmann SA, Schaaf JA, Strube MJ. Differences in the Gait Characteristics of Patients With Diabetes and Peripheral Neuropathy Compared With Age-Matched Controls. Physical Therapy 1994; 74(4).

60. Kaufman KR, Hughes C, Morrey BF, Morrey M, An KN. Gait characteristics of patients with knee osteoarthritis. J Biomech 2001; 34(7): 907-15.

61. Pirker W, Katzenschlager R. Gait disorders in adults and the elderly : A clinical guide. Wien Klin Wochenschr 2017; 129(3-4): 81-95.

62. Verlinden VJ, van der Geest JN, Hoogendam YY, Hofman A, Breteler MM, Ikram MA. Gait patterns in a community-dwelling population aged 50 years and older. Gait Posture 2013; 37(4): 500-5.

63. Floegel TA, Florez-Pregonero A, Hekler EB, Buman MP. Validation of Consumer-Based Hip and Wrist Activity Monitors in Older Adults With Varied Ambulatory Abilities. J Gerontol A Biol Sci Med Sci 2016.

64. Beevi FH, Miranda J, Pedersen CF, Wagner S. An Evaluation of Commercial Pedometers for Monitoring Slow Walking Speed Populations. Telemed J E Health 2016; 22(5): 441-9. 
65. Martin JB, Krč KM, Mitchell EA, Eng JJ, Noble JW. Pedometer accuracy in slow walking older adults. Int J Ther Rehabil 2012; 19(7): 387-93.

66. Cyarto EV, Myers A, Tudor-Locke C. Pedometer accuracy in nursing home and community-dwelling older adults. Med Sci Sports Exerc 2004; 36(2): 205-9.

67. Nelson MB, Kaminsky LA, Dickin DC, Montoye AH. Validity of Consumer-Based Physical Activity Monitors for Specific Activity Types. Med Sci Sports Exerc 2016; 48(8): 1619-28.

68. Van Remoortel H, Giavedoni S, Raste Y, et al. Validity of activity monitors in health and chronic disease: a systematic review. Int J Behav Nutr Phys Act 2012; 9: 84.

69. Epstein DA, Kang JH, Pina LR, et al. Reconsidering the device in the drawer: lapses as a design opportunity in personal informatics. Ubicomp; 2016 Sep 12-16; Heidelberg, Germany.

70. Lazar A, Koehler C, Tanenbaum J, et al. Why we use and abandon smart devices. Ubicomp; 2015 Sep 711; Osaka, Japan.

71. Harrison D, Marshall P, Bianchi-Berthouze N, et al. Activity tracking: barriers, workarounds and customisation. Ubicomp; 2015 Sep 7-11; Osaka, Japan.

72. Shih PC, Han K, Poole ES, et al. Use and adoption challenges of wearable activity trackers. iConference; 2015 Mar 24-27; Newport Beach, California, USA.

73. Armağan K, Çiğdem E. Design for physical activity: design aspects of wearable activity trackers. International Conference of Engineering Design; 2015 Jun 27-30; Milano, Italy

74. Fausset CB, Mitzner TL, Price CE, Jones BD, Brad Fain W, Rogers WA. Older Adults' Use of and Attitudes toward Activity Monitoring Technologies. Proceedings of the human factors and ergonomics society; 2013.

75. O'Brien T, Troutman-Jordan M, Hathaway D, Armstrong S, Moore M. Acceptability of wristband activity trackers among community dwelling older adults. Geriatric nursing 2015; 36(2 Suppl): S21-5.

76. Rasche P, Schäfer K, Theis S, Bröhl C, Wille M, Mertens A. Age-related usability investigation of an activity tracker. International Journal of Human Factors and Ergonomics 2016; 4(3/4): 187.

77. Mercer K, Giangregorio L, Schneider E, Chilana P, Li M, Grindrod K. Acceptance of Commercially Available Wearable Activity Trackers Among Adults Aged Over 50 and With Chronic IIIness: A Mixed-Methods Evaluation. JMIR Mhealth Uhealth 2016; 4(1): e7.

78. Rosenberg D, Kadokura EA, Bouldin ED, et al. Acceptability of Fitbit for physical activity tracking within clinical care among men with prostate cancer. AMIA Annu Symp Proc; 2016 Nov 4-8; Washington, USA.

79. Limburg Meet. Limburg Meet. URL: https://www.limeconnect.nl/ (accessed: 2021-03-08) 




\section{Chapter

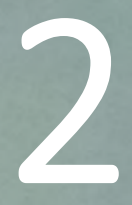

Counting steps in activities of daily living in people with a chronic disease using nine commercially available fitness trackers: cross-sectional validity study 


\section{Abstract}

\section{Background}

Measuring physical activity with commercially available activity trackers is gaining popularity. People with a chronic disease can especially benefit from knowledge about their physical activity pattern in everyday life since sufficient physical activity can contribute to wellbeing and quality of life. However, no validity data are available for this population during activities of daily living.

\section{Objective}

The aim of this study was to investigate the validity of 9 commercially available activity trackers for measuring step count during activities of daily living in people with a chronic disease receiving physiotherapy.

\section{Methods}

The selected activity trackers were Accupedo (Corusen LLC), Activ8 (Remedy Distribution Ltd), Digi-Walker CW-700 (Yamax), Fitbit Flex (Fitbit inc), Lumoback (Lumo Bodytech), Moves (ProtoGeo Oy), Fitbit One (Fitbit inc), UP24 (Jawbone), and Walking Style $X$ (Omron Healthcare Europe BV). In total, 130 persons with chronic diseases performed standardized activity protocols based on activities of daily living that were recorded on video camera and analyzed for step count (gold standard). The validity of the trackers' step count was assessed by correlation coefficients, t tests, scatterplots, and BlandAltman plots.

\section{Results}

The correlations between the number of steps counted by the activity trackers and the gold standard were low (range: -0.02 to 0.33 ). For all activity trackers except for Fitbit One, a significant systematic difference with the gold standard was found for step count. Plots showed a wide range in scores for all activity trackers; Activ8 showed an average overestimation and the other 8 trackers showed underestimations.

\section{Conclusions}

This study showed that the validity of 9 commercially available activity trackers is low measuring steps while individuals with chronic diseases receiving physiotherapy engage in activities of daily living. 


\section{Introduction}

The use of activity tracking to self-monitor physical activity is gaining popularity. In 2015, 1 out of 3 Dutch inhabitants was using apps, wearables, or activity trackers. ${ }^{1}$ Physical activity is the most popular variable measured with these devices followed by nutrition, weight, and body functions (e.g., blood pressure). ${ }^{1}$ Initially, these activity trackers were developed for athletes and the healthy population, but they could potentially also be useful in treating people with medical conditions (e.g., physiotherapy treatments). The Royal Dutch Society for Physical Therapy composed physical activity intervention guidelines for the most common chronic diseases seen by a physiotherapist ${ }^{2}$ : cardiovascular disease ${ }^{3}$, diabetes mellitus ${ }^{4}$, chronic obstructive pulmonary disease $(\mathrm{COPD})^{5}$, chronic pain $^{6}$, cancer $^{7}$, and osteoarthritis. ${ }^{8}$ In all these guidelines, it is recommended to objectively measure the physical activity level of a patient outside of guided therapy. ${ }^{2}$ Frequently used measurement tools by physiotherapists are questionnaires or diaries, but they have limited reliability and validity, tend to overestimate most activities while underestimating low intensity activities, and are time consuming to fill out. ${ }^{9,10}$ For patients and physiotherapists, more objective and feasible measurement tools are useful, and activity trackers seem to be a good alternative. ${ }^{11}$

To provide guidance in choosing an appropriate activity tracker for people with a chronic disease, we performed a literature search on the validity of activity trackers, preferably commercially available ones. The following criteria were taken into account. First, step count was considered to be the most important outcome, since it is specific to ambulation and easily interpreted by patients and physiotherapists. ${ }^{11}$ Second, people with a chronic disease should be the target population of the study, as they often have impaired ambulatory abilities (e.g., shuffling) ${ }^{12}$, and activity trackers may measure incorrectly due to these altered walking patterns. ${ }^{13-15}$ Third, activities of daily living should be assessed (no laboratory settings), as insight into these specific activities (e.g., vacuum cleaning, walking stairs) is needed to monitor and coach participants in daily life, and activity trackers are not able to measure validly during low walk speeds $(<0.8 \mathrm{~m} / \mathrm{s})^{16}$, which is often the case in activities of daily living. Last, published articles were screened on standardization of the performed activities of daily living by means of an activity protocol.

Although the literature on clinometric quality of commercially available activity trackers is growing ${ }^{17-19}$, only a few recent studies were found in which almost all criteria were met (validity of step count of commercially available activity trackers during free living conditions). ${ }^{19,20}$ However, the target population in those studies consisted of healthy participants. 
Remoortel et al. ${ }^{18}$ recently published a literature review regarding validity and reliability of activity trackers in people with a chronic disease. It was confirmed that most commercially available activity trackers have been studied in healthy populations ${ }^{17,19-21}$, and little is known about which types of activity trackers provide valid results in people with chronic diseases. In their review, they found that only 12 of the 134 studies on validity of activity trackers included people with a chronic disease. ${ }^{18}$ of the 12 identified studies, only 3 evaluated activities of daily living (free living or an activity protocol) in people with a chronic disease ${ }^{22-24}$; however, these studies only tested noncommercially available activity trackers and mainly evaluated energy expenditure instead of step count. Results from other studies with participants with chronic diseases are not generalizable to daily practice because they did not have step count as the primary outcome (e.g., mostly energy expenditure $)^{25-31}$, involved only walking and no other activities of daily living ${ }^{32-35}$, or free living conditions were not protocoled (e.g., cardiac patients ${ }^{36}$ and patients with COPD $^{37}$ or cancer $^{38}$ ).

As stated before, for both people with a chronic disease and their therapists, insight into physical activity level and patterns outside of therapy are very relevant. Since no article was found that matched our criteria, we decided to validate 9 potential trackers ourselves in people with a chronic disease.

The main aim of this study was to investigate the validity of 9 selected commercially available activity trackers for measuring step count in people with a chronic disease receiving physiotherapy during a selected set of activities of daily living. Results from this study should provide guidance in choosing the right activity tracker for people with a chronic disease.

\section{Methods}

\section{Study design}

A cross-sectional validity study with 9 activity trackers was performed in patients with chronic diseases. The data collection took place over a 1-year period. All participants provided written informed consent. This study was approved by the local ethics boards (Atrium-Orbis-Zuyd Medical Ethical Committee, 15-N-48; Adelante Medical Ethical Committee, MEC-15-07).

\section{Participants}

Participants were recruited from 2 physiotherapy practices (Fysiotherapie Schaesberg and ParaMedisch Centrum Zuid) and a rehabilitation center (Adelante Zorggroep) in the 
Netherlands. Patients were included if they were aged 18 years and older and diagnosed with at least 1 of the following chronic diseases: cardiovascular disease, COPD, diabetes mellitus, chronic pain, cancer, or osteoarthritis. Exclusion criteria were insufficient understanding of the Dutch language, use of a walking aid, and asymmetrical gait (e.g., stroke). A power calculation was conducted, and a minimum of 57 participants with an equal spread among the 6 chronic subpopulations was considered to be sufficient for a validity study. ${ }^{39}$

\section{Activity trackers}

Researchers and physiotherapists agreed to the following selection criteria for commercially available activity trackers: costs less than $€ 150$ (US \$185), no monthly costs for a subscription, real-time feedback on the tracker to the user, measures number of steps, and no chest strap to perform heart rate measurements. To ensure that the scope of different system requirements was covered, trackers were randomly selected in a second round based on the following criteria: a variety of wearing places (e.g., belt, wrist) and types of activity trackers (e.g., pedometers, accelerometers). Hence, 9 activity trackers were selected: Accupedo (Corusen LLC), Activ8 (Remedy Distribution Ltd), DigiWalker CW-700 (Yamax), Fitbit Flex (Fitbit Inc), Lumoback (Lumo Bodytech), Moves (ProtoGeo Oy), Fitbit One (Fitbit Inc), UP24 (Jawbone), and the Walking Style X (Omron Healthcare Europe BV) (Table 2.1).

Table 2.1 Selected commercially available activity trackers used in this validity study.

\begin{tabular}{|c|c|c|c|c|}
\hline Activity tracker & Manufacturer & Type & $\begin{array}{l}\text { Wearing } \\
\text { position }\end{array}$ & Outcome variables \\
\hline Accupedo & Corusen LLC & App & Belt & Number of steps \\
\hline Activ8 & $\begin{array}{l}\text { Remedy } \\
\text { Distribution Ltd }\end{array}$ & Accelerometer & Trouser pocket & $\begin{array}{l}\text { Number of steps; time spent lying, } \\
\text { sitting, standing, walking, running, } \\
\text { and cycling; active minutes }\end{array}$ \\
\hline Digi-Walker CW-700 & Yamax Corp & Pedometer & Wrist & Number of steps, active minutes \\
\hline Flex & Fitbit Inc & Accelerometer & Wrist & Number of steps, active minutes \\
\hline Lumoback & Lumo Bodytech & Accelerometer & Lower back & $\begin{array}{l}\text { Number of steps; time spent lying, } \\
\text { sitting, standing, walking, running, } \\
\text { and cycling; active minutes; } \\
\text { number of sit-to-stand transitions }\end{array}$ \\
\hline Moves & ProtoGeo Oy & App & Trouser pocket & Number of steps, active minutes \\
\hline One & Fitbit Inc & Accelerometer & Belt & Number of steps, active minutes \\
\hline UP24 & Jawbone & Accelerometer & Wrist & Number of steps, active minutes \\
\hline Walking Style X & $\begin{array}{l}\text { Omron Healthcare } \\
\text { Europe BV }\end{array}$ & Pedometer & Belt & Number of steps, active minutes \\
\hline
\end{tabular}




\section{Data collection and procedure}

Participants were measured in either of the physiotherapy practices or the rehabilitation center. Baseline characteristics were reported (gender, age, body weight, height, diagnosed chronic disease) by 1 of the 10 participating physiotherapists or a psychologist. For participants with COPD, the Global Initiative for Chronic Obstructive Lung Disease stage ${ }^{40}$ was specified. For participants with osteoarthritis, a differentiation was given for lower extremity (toe, ankle, knee, hip), upper extremity (finger, wrist, elbow, shoulder), and cervical and lower spine. In participants with cancer, curative and palliative treatments were distinguished. Two questionnaires were completed with the participant. The Cumulative IIIness Rating Scale (CIRS) was used to indicate the number and severity of comorbidities. ${ }^{41,42}$ For an impression of the participant's physical activity level, a brief physical activity assessment tool was used to determine whether the participant was sufficiently active. ${ }^{43}$ After completing the questionnaires a 10-meter walk test (10MWT) was performed 3 times to determine the average comfortable walk speed of the participant. ${ }^{44}$ Thereafter, participants were fitted with 3 or 4 activity trackers, chosen at random, and asked to perform the activity protocol.

\section{Activity protocol}

Tasks representing activities of daily living from protocols in previous validation studies ${ }^{24,29,45,46}$ were used to create the protocol for this study (Table 2.2). In order to match the participants' physical activity capacity, 2 versions of the protocol were developed, assuming that the length of the protocol had no influence on the validity of the trackers. The short version of the protocol did not include lying on a bed, vacuum cleaning on the spot, and 3 additional periods of standing, shortening the execution time of the protocol by 9 minutes. Activity trackers not able to classify different postures were used in the short protocol. Participants were given extra resting periods during the protocol of they needed them.

Step count was collected from the activity trackers before and directly after the protocol. The entire activity protocol was recorded on video camera, focusing only on the lower extremity for privacy reasons. The video recordings were used to determine the number of steps taken by each participant. Step count was manually counted using a digital step counter (gold standard). A person was considered to make a step when the entire foot was lifted from the floor and was placed back on the floor again (detailed information is published elsewhere ${ }^{39}$ ). The 7 raters involved used a standardized written assessment protocol and were trained by 1 researcher beforehand. The first 2 video recording assessments per rater were checked by the researcher (DU) to secure standardization of the measurement method. 
Table 2.2 The developed activity protocol based on principles and free living tasks from other protocols.

\begin{tabular}{lll}
\hline Activity type & $\begin{array}{l}\text { Duration of activity, repetitions or } \\
\text { walking distance }\end{array}$ & $\begin{array}{l}\text { Included in short } \\
\text { version }\end{array}$ \\
\hline Standing & 1 minute & Yes \\
Simulated cleaning of windows & 1 minute & Yes \\
Walking weaving around cones & 7 meters & Yes \\
Sitting in a chair & 2 minutes & Yes \\
Standing & 1 minute & No \\
Vacuum cleaning on the spot & 1 minute & No \\
Vacuum cleaning while walking & 1 minute & Yes \\
Walking weaving around cones & 7 meters & Yes \\
Walking up and down stair (3 or 4 steps & 3 times & Yes \\
Lifting a 1-kg objects and placing it on a table & 1 minute & Yes \\
Walking in a straight line & 7 meters & Yes \\
Lying in a bed & 6 minutes & No \\
Sitting in a chair & 5 minutes & Yes \\
Standing & 1 minute & No \\
Walking in a line while carrying a shopping bag (2.5 kg) & 7 meters 2 times & Yes \\
Walking sideways along a 2 meter kitchen counter & 2 ways 3 times & Yes \\
Standing & 30 seconds & No \\
Walking in a straight line & 7 meters & Yes \\
Cycling (50 to 60 rpms a at 30 watts) & 3 minutes & Yes \\
Total time & 28 to 33 minutes & 19 to 24 minutes \\
\hline
\end{tabular}

${ }^{\mathrm{a}}$ Revolutions per minute.

\section{Data analysis}

Data analysis was performed using the SPSS Statistics version 23.0 (IBM Corp). Descriptive statistics of the participant characteristics were presented as raw data and percentages for the categorical variables gender, diagnosed disease, and physical activity (sufficient/insufficient) ${ }^{43}$ and as means and standard deviations for the continuous variables age, CIRS score, and average walk speed.

The video recordings of the activity protocols were analyzed by at least 1 researcher. One-tenth randomly chosen video recordings were analyzed by a second researcher to assess intra observer reliability of our gold standard. This was assessed by intraclass correlation coefficients (ICCS; 2-way random, absolute agreement) and Bland-Altman plots including limits of agreement. ${ }^{47}$ It was hypothesized that there would be a strong correlation $(r>0.90){ }^{48}$

The validity of the activity trackers was assessed in multiple ways. To gain insight into step count distribution, descriptive statistics and scatterplots were used for all trackers. To gain insight into the strength of the relation between measured steps by the activity trackers and the gold standard, Pearson correlation coefficients were calculated. It was 
hypothesized that there would be at least a moderate correlation $(r>0.40) .{ }^{48}$ To assess systematic differences between the activity trackers and the gold standard, paired samples $t$ tests were used. With a power of $80 \%$, a $P$ value below 0.05 was considered to be of statistical significance. To examine the level of agreement between the activity trackers and the gold standard, Bland-Altman plots were constructed with their associated $95 \%$ limits of agreement. ${ }^{49}$

To assess if there were difference between the chronic diseases, visual inspection of the scatterplots were performed. To assess if there were systematic differences between the average mean differences of the short and long protocols, independent $t$ tests were used. To test if there was a systematic difference in the mean difference between the gold standard and the activity tracker between the short and long protocols, a paired sample $t$ test was used in the case of normally distributed data. In the case of missing data, pairwise deleting was applied.

\section{Results}

\section{Participant characteristics}

A total of 130 participants with chronic diseases participated in this validation study (Table 2.3). Cardiovascular disease, chronic pain, and osteoarthritis were the most prevalent single conditions, and $26.4 \%$ (34/130) of the population had multimorbidity. The combinations occurring most often were osteoarthritis with chronic pain $(6 / 34$, $17.6 \%)$, osteoarthritis and diabetes (4/34, 11.7\%), and COPD and diabetes (3/34, 8.8\%). Approximately $60 \%(75 / 130)$ of the participants were sufficiently physically active in their daily life according to the physical activity assessment tool. Of the included COPD patients, $7.7 \%(1 / 14)$ were diagnosed with stage 1 COPD, 35.7\% (5/14) with stage 2, $42.9 \%(6 / 14)$ with stage 3 , and $14.3 \%$ (2/14) with stage 4 . Of the cancer patients, $82.6 \%$ $(19 / 23)$ had a curative treatment and $17.4 \%(4 / 23)$ a palliative treatment. The affected joints in osteoarthritis were almost equally spread in upper extremity $(22 / 33,66.7 \%)$, spine $(24 / 33,72.7 \%)$, and lower extremity $(23 / 33,69.7 \%)$. There were 2 missing values for gender, diagnosed disease, resting heart rate and body mass index (BMI) (2/130, $2.6 \%)$, and 3 missing values for age (3/130, 3.9\%). There was 1 missing value for the number of steps from the Lumoback (1/51, 5.1\%) and 1 from the Accupedo (1/50, 5\%). 
Table 2.3 Characteristics of the included population.

\begin{tabular}{|c|c|}
\hline Characteristics & Participants $(n=130)$ \\
\hline Gender, male, n (\%) & $55(43.6)$ \\
\hline Age, years, mean (SD) & $61.5(11.1)$ \\
\hline Body mass index, $\mathrm{kg} / \mathrm{m}^{2}$, mean (SD) & $27.7(5.2)$ \\
\hline \multicolumn{2}{|l|}{ Blood pressure, mm Hg, mean (SD) } \\
\hline Systolic & $136.2(20.3)$ \\
\hline Diastolic & $80.3(9.7)$ \\
\hline Resting heart beat, beats per minute, mean (SD) & $74.0(12.2)$ \\
\hline Transcutaneous oxygen saturation, $\%$, mean (SD) & $96.4(2.3)$ \\
\hline \multicolumn{2}{|l|}{ Diagnosed disease, $\mathrm{n}(\%)$} \\
\hline Cardiovascular disease & $20(15.2)$ \\
\hline Chronic obstructive pulmonary disease & $15(11.4)$ \\
\hline Diabetes mellitus & $8(6.1)$ \\
\hline Cancer & $15(11.4)$ \\
\hline Osteoarthritis & $18(14.4)$ \\
\hline Chronic pain & $19(14.4)$ \\
\hline Combination & $34(27.3)$ \\
\hline Comorbidity, $\mathrm{CIRS}^{\mathrm{a}} 0$ to 52 , mean (SD) & $6.2(3.9)$ \\
\hline Average walk speed ${ }^{b}(\mathrm{~m} / \mathrm{s})$ mean $(\mathrm{SD})$ & $1.30 .3)$ \\
\hline Sufficient total activity, $\mathrm{n}(\%)^{\mathrm{C}}$ & $74(56.4)$ \\
\hline Physical activity level (0 to 8 ), mean (SD) & $3.8(2.4)$ \\
\hline Physical activity with moderate intensity ( 0 to 4 ), mean (SD) & $1.6(1.6)$ \\
\hline Physical activity with vigorous intensity (0 to 4), mean (SD) & $2.2(1.5)$ \\
\hline
\end{tabular}

\section{Interobserver reliability}

The interobserver reliability of the gold standard, calculated in the random sample, was high (ICCagreement $0.98, P<0.001,95 \% \mathrm{Cl} 0.96$ to 0.99). There was no substantial offset (SEMagreement=81.6) and the Bland-Altman plots showed no systematic differences between the observers (with narrow limits of agreement: -35.3 to 30.8 steps).

\section{Step count}

Step count for the gold standard and each tracker are shown in Table 2.4. The average total number of steps during the short and long activity protocols counted by the gold standard was 405.4 (SD 84.7). The average total number of steps for the short protocol was 327.7 (SD 54.3) and the average total number of steps for the long protocol was 446.6 (SD 58.6). There was no significant difference between the mean difference (gold standard versus activity tracker) in the short and long protocols. For all activity trackers except for the Activ8, the mean difference with the gold standard was lower than zero, which indicated an underestimation of the total number of steps. The mean difference 
between the tracker and gold standard varied from -29.7 (SD 155.10) for the Fitbit One to 252.4 (SD 129.0) for the Digi-Walker CW-700. Overall, data distribution showed a wide range of observations for all activity trackers. There were no differences found per chronic disease compared to the whole population. Scatter plots of the Fitbit One, DigiWalker CW-700, and Activ8 are presented in Figures 2.1-2.3 to give examples of data distribution.

Table 2.4 Descriptive statistics of step count by activity tracker compared to the gold standard.

\begin{tabular}{lcccc}
\hline Activity tracker & $\begin{array}{c}\text { Number of } \\
\text { participants }\end{array}$ & $\begin{array}{c}\text { Average mean difference } \\
\text { in step count (SD) }\end{array}$ & $\begin{array}{c}\text { Average median difference in } \\
\text { step count (25 to } 75 \\
\text { percentile) }\end{array}$ & $\begin{array}{c}\text { Limits of } \\
\text { agreement } \\
\text { (lower bound- } \\
\text { upper bound) }\end{array}$ \\
\hline Accupedo & 50 & $-176.3(132.1)$ & $-174.5(-251.0$ to -102.5$)$ & -435.2 to 82.7 \\
Activ8 & 62 & $107.3(251.9)$ & $126.0(30.5$ to 243.5$)$ & -471.3 to 721.0 \\
Digi-Walker CW-700 & 52 & $-284.5(129.0)$ & $-253.0(-383.0$ to -169.0$)$ & -537.4 to -31.7 \\
Fitbit Flex & 47 & $-93.5(126.7)$ & $-111.0(-167.0$ to 3.0$)$ & -326.9 to 123.7 \\
Lumoback & 51 & $-178.5(96.0)$ & $-168.0(-205.5$ to $-117,0)$ & -366.9 to 9.3 \\
Moves & 48 & $-146.6(216.3)$ & $-215.0(-279.5$ to -89.3$)$ & -570.5 to 277.4 \\
Fitbit One & 49 & $-29.7(155.1)$ & $-8.0(-160.0$ to 128.0$)$ & -367.8 to 308.6 \\
UP24 & 49 & $-252.4(104.7)$ & $-266.0(-327.0$ to -176.5$)$ & -457.7 to -47.2 \\
Walking Style X & 50 & $-204.4(117.7)$ & $-206.5(-256.0$ to -105.0$)$ & -438.0 to 27.2 \\
\hline
\end{tabular}

${ }^{a}$ Activity tracker minus gold standard.

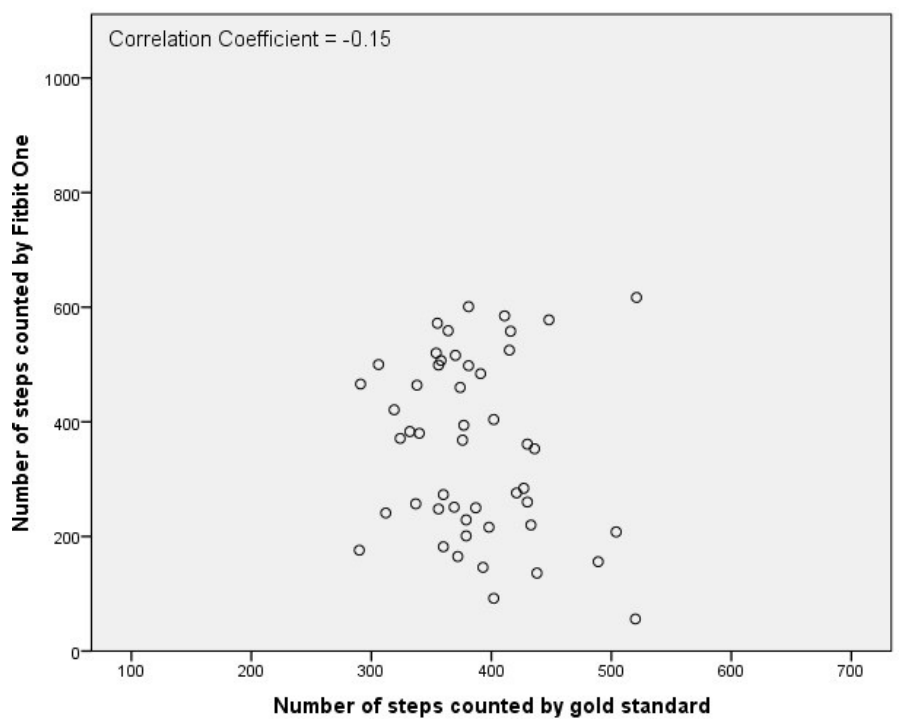

Figure 2.1 Scatterplot of the number of steps counted by Fitbit One and the gold standard. 


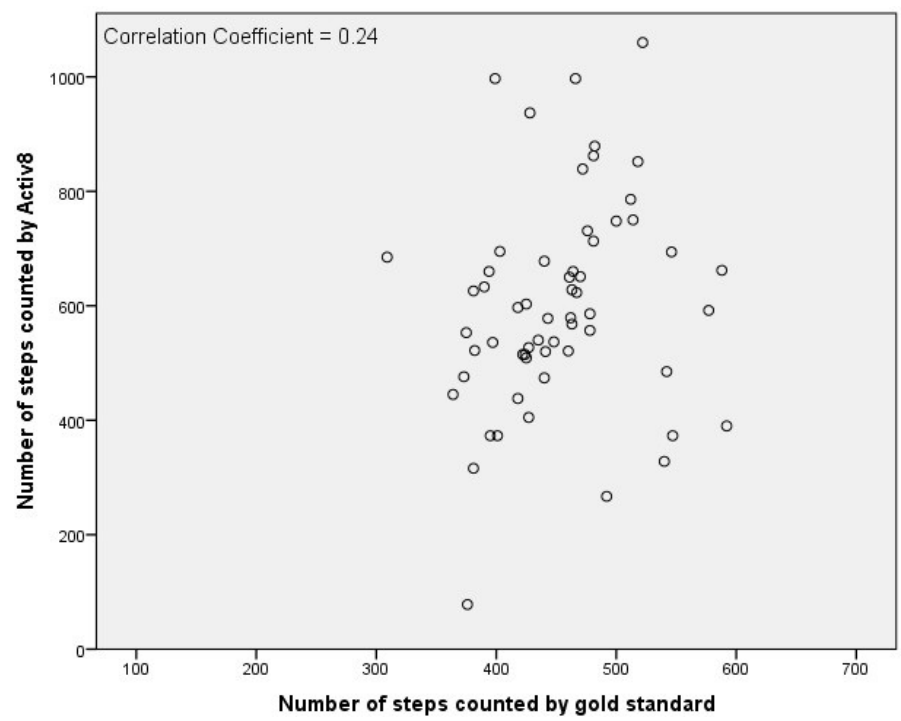

Figure 2.2 Scatterplot of the number of steps counted by Activ8 and the gold standard.

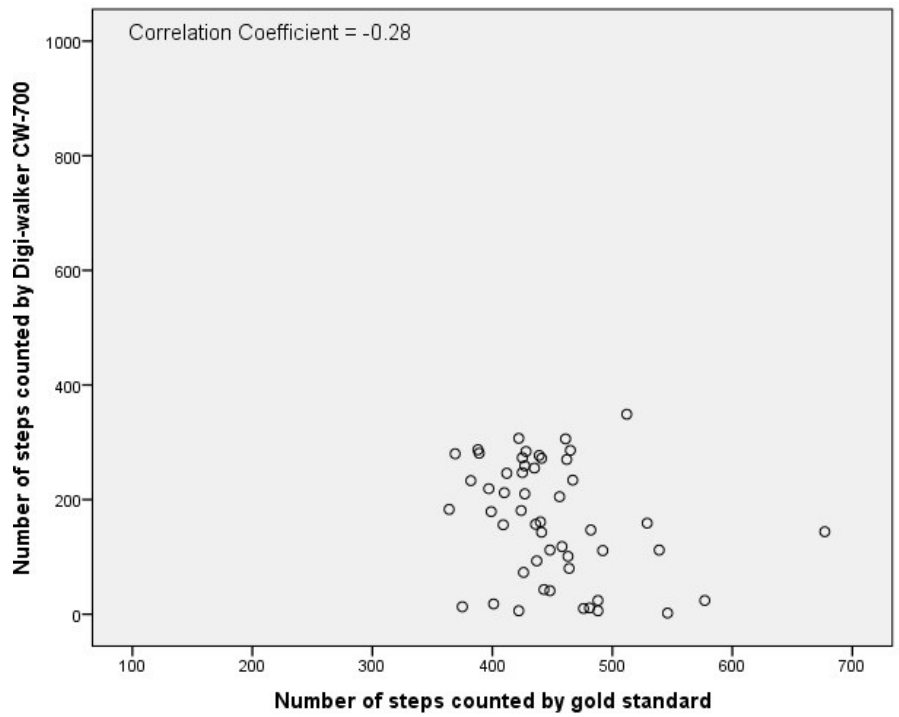

Figure 2.3 Scatterplot of the number of steps counted by Digi-walker CW-700 and the gold standard. 


\section{Strength of the relation and systematic difference}

The correlation between the number of steps measured by the activity trackers and the gold standard was weak for all activity trackers ranging from $r=-0.02$ for the Moves to $r=-0.33$ for the Digi-Walker CW-700 (Table 2.5). The average underestimation of all trackers and the average overestimation of the Activ8 revealed a significant systematic difference with the gold standard for step count, expect for the Fitbit One ( $P=0.35)$.

Table 2.5 Correlation coefficient of the activity trackers and the gold standard for step count.

\begin{tabular}{lcc}
\hline Activity tracker & Correlation coefficient $(P$ value $)$ & t value $(P$ value $)$ \\
\hline Accupedo & $0.32(0.02)$ & $-9.4(<0.001)$ \\
Activ8 & $0.24(0.06)$ & $-3.9(0.001)$ \\
Digi-Walker CW-700 & $-0.33(0.02)$ & $-6.2(<0.001)$ \\
Flex & $0.31(0.04)$ & $-5.1(<0.001)$ \\
Lumoback & $0.19(0.20)$ & $-6.2(<0.001)$ \\
Moves & $-0.02(0.88)$ & $-3.4(0.001)$ \\
One & $-0.15(0.30)$ & $-0.9(0.35)$ \\
UP24 & $0.09(0.52)$ & $-6.9(<0.001)$ \\
Walking StyleX & $0.25(0.08)$ & $-12.3(<0.001)$ \\
\hline
\end{tabular}

\section{Level of agreement}

In all plots the limits of agreement are high, with the highest limits of agreement ( -471.3 to 721.0) for the Activ8 (Table 2.4). In the plots, 2 trends are visible: either an over- and underestimation of the number of steps during the activity protocols as shown in Figures 2.4 and 2.5 (e.g., Fitbit One and Activ8) or an underestimation of the number of steps only, as shown in Figure 2.6 (e.g., Digi-Walker CW-700). Depending on the height of step count, overestimation or underestimation was shown. Overestimation became more pronounced when participant took more steps and vice versa.

\section{Systematic difference between short and long protocols}

Only the Walking Style X, Accupedo, and Fitbit Flex were used in both protocols. For all trackers, there were no systematic differences found for the average mean difference in step coun between the short and long protocols. 


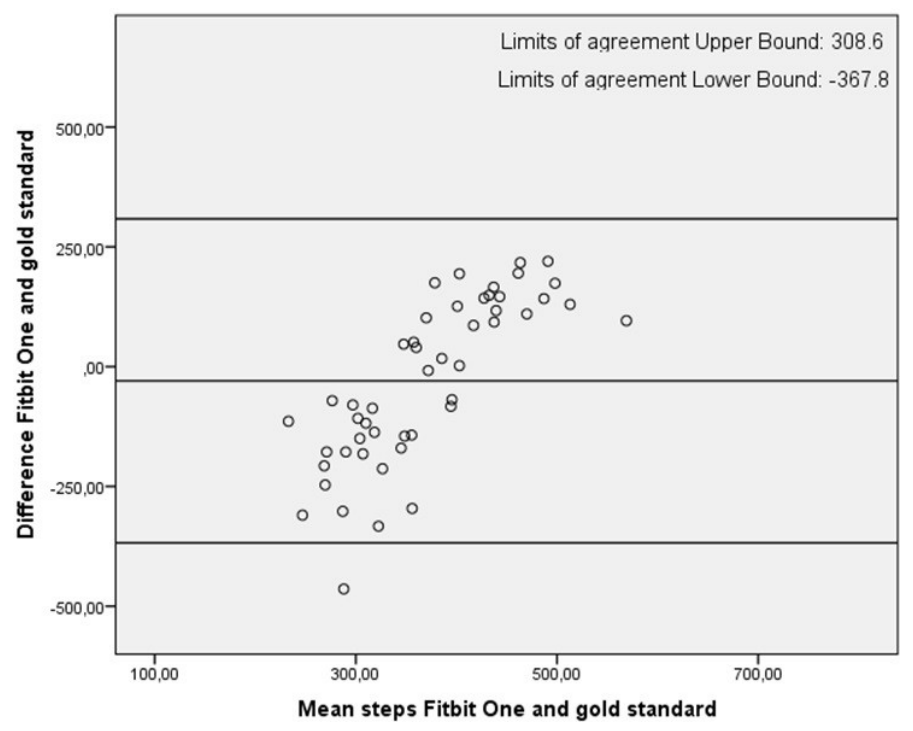

Figure 2.4 Bland-Altman plot of Fitbit One and the gold standard.

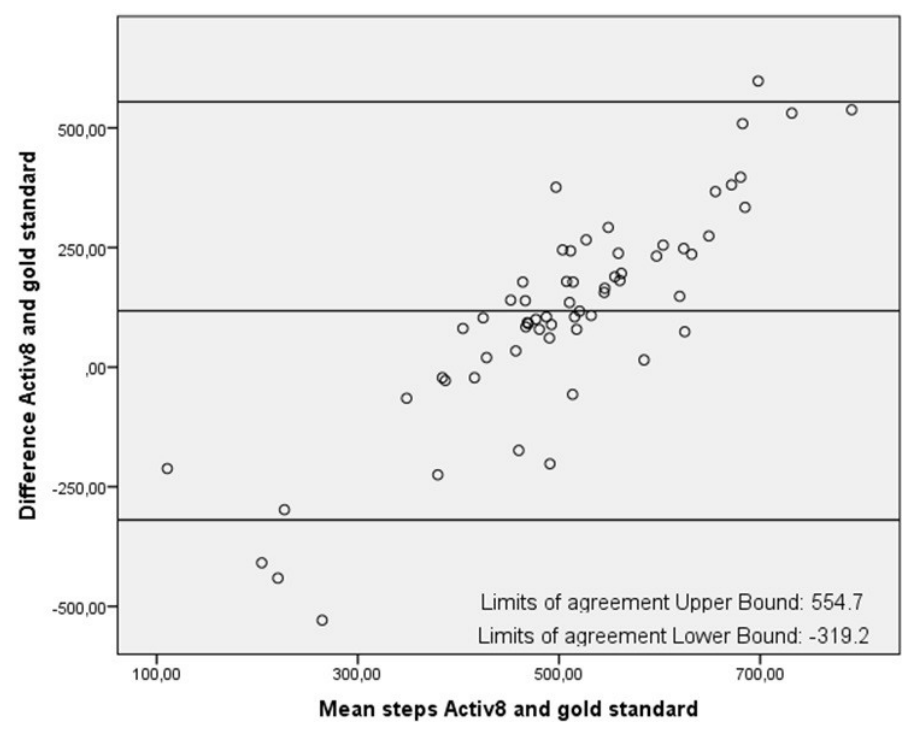

Figure 2.5 Bland-Altman plot of the Aciv8 and the gold standard. 


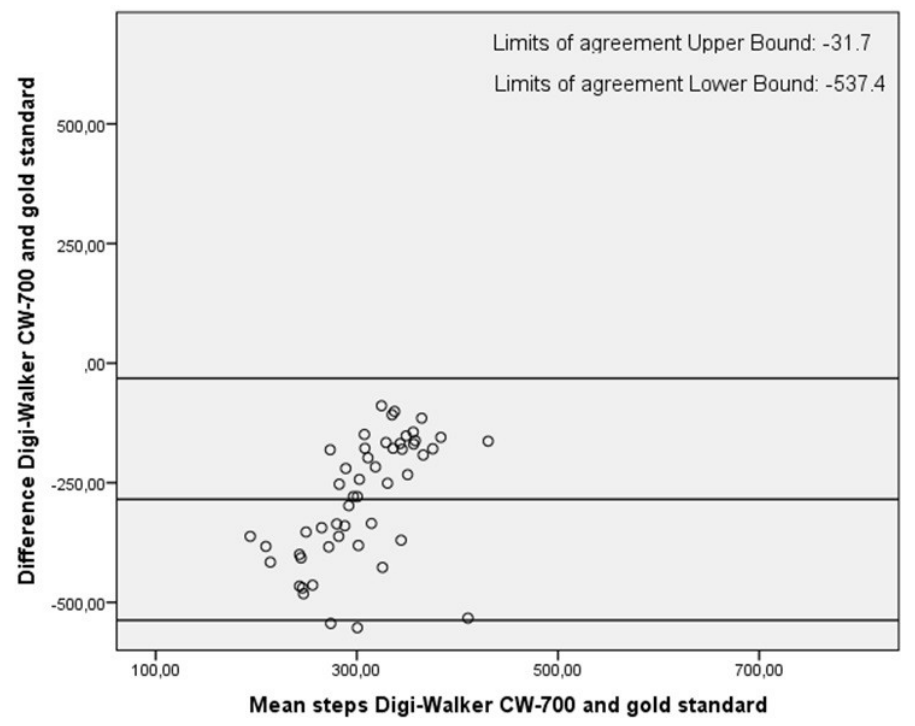

Figure 2.6 Bland-Altman plot of Digi-Walker CW-700 and the gold standard.

\section{Discussion}

\section{Principal findings}

The results of this study showed that none of 9 selected commercially available activity trackers was valid for measuring steps while individuals engage in activities of daily living among a diverse group of patients with various chronic diseases receiving physiotherapy in the Netherlands.

All activity trackers in this study had an average underestimation in step count except the Activ8, which overestimated step count. The Digi-Walker CW-700 and Lumoback consistently underestimated step count in every participant, while the other activity trackers had a combination of under- and overestimation. For all trackers, the correlations between step count measured by the activity trackers and the observed steps were low. On group level, the Fitbit One seemed to be the best activity tracker due to its low mean difference; however, on individual basis the scatter and Bland-Altman plots showed a large under- and overestimation in step count.

Several studies have shown that a low walking speed decreases the validity of activity trackers. ${ }^{12,16,50,51}$ For an activity tracker to measure the number of steps correct, a walking speed of $0.8 \mathrm{~m} / \mathrm{s}$ is required. All of our participants walked faster than $0.8 \mathrm{~m} / \mathrm{s}$ 
during the 10MWT; therefore, their walking speed should have been sufficient for an accurate measurement by the activity tracker. However, the activity protocol consisted of different household tasks such as vacuum cleaning and washing windows, resulting in a walking speed below $0.8 \mathrm{~m} / \mathrm{s}$. Recently, Nelson et al. ${ }^{52}$ published the results of a validation study in which the Fitbit Flex and Fitbit One were assessed during activities of daily living in a healthy population. They concluded that these activity trackers underestimate step count by $60 \%$ during household activities, but during walking activities the percentage error was within $4 \%$. Nelson et al concluded that this difference might come from slow ambulation speed and shuffling during these household activities. Although the populations differ, these results confirm the findings in our study. Our standardized activity protocol was based on earlier protocols with activities of daily living in COPD patients ${ }^{24,29,45}$ and is therefore comparable to real-life performance of people with a chronic disease. Our protocol consisted of various activities of short duration, since this is more comparable to the performance of the activities in the daily life of people with a chronic disease. Since the study population had a limited physical activity capacity and more fatigue, pain, and possibly dyspnea, the requirements of the longer protocol might not have matched their physical possibilities and might not represent the daily life of people with a chronic disease. During the execution of the study, all patients were able to perform the entire protocol, and no patients had to be excluded due to the effort required by the protocol. However, the results of our study contradict studies performed in healthy populations in which the 9 tested activity trackers showed good validity in free-living situations. ${ }^{19,20}$ An explanation could be that the walking speed is faster during free-living situations because patients perform more walking activities in comparison to an activity protocol with activities of daily living. To the authors knowledge, only 1 validation study was performed in people with a chronic disease (cardiac patients) using one of the assessed activity trackers (Fitbit Flex ${ }^{36}$ ). This study concluded that there was a high correlation between the Fitbit Flex and the Actigraph for step count $(r=0.95)$.

\section{Limitations and strengths}

The chosen activity trackers were the most up-to-date activity trackers at the time. During this study, several updates were released for the chosen activity trackers (mostly the exterior instead of the algorithm), and several new activity trackers were brought to the market. But the chosen activity trackers are still the most popular and most used activity trackers currently available. ${ }^{53-55}$

In this study design, 2 activity protocols were used. It was assumed that the length of the protocol had no influence on the trackers' validity because the removed activities were 
activities that didn't require walking. There were no systematic differences in average mean difference in step count between the short and long protocols.

For determining the validity of the step count, the definition of a step is very important. In this study, a step was defined as when the entire foot was lifted from the floor and placed back on the floor again. However, shuffling is frequently seen in elderly populations and in people with a chronic disease. ${ }^{12}$ If shuffling steps were included in our analysis (thus more steps during the protocol), more underestimation of the activity trackers would be likely, implying an even lower validity. In this study, it wasn't possible to report validity of the activity trackers per activity. All selected activity trackers were commercially available trackers, and thus their algorithms and time slots were not available on request. Without specific information regarding (at least) the timeslots, it was not possible to disentangle time per activity.

In this study, we used different methods for evaluation of the validity. By using these different methods, insight was gained on validity on both group and individual levels. Validity on individual level is important for daily practice for patients and therapists. We included the $P$ value for the correlation coefficient; however, this is a measurement on group level and not on individual level. Therefore, the significant correlations are not clinically relevant. Moreover, the 3 significant correlations (Accupedo, Digi-Walker CW700 , and the Flex) are still considered weak correlations. ${ }^{48}$

A strength of this study is the use of observed steps as gold standard. The high reliability of this gold standard assures very little systematic bias in the analysis method. The chronic diseases included in this study are those most frequently seen by physiotherapists in the Netherlands ${ }^{2}$, implying that the study results might be generalizable to a broad population. However, this should be confirmed by including a broader range of patients with chronic diseases not limited to primary care physical therapy practices.

\section{Clinical relevance}

Guidelines recommend objectively measuring the physical activity level of a patient outside of guided therapy. ${ }^{2}$ However, underestimation or overestimation of physical activity by an activity tracker is not desirable. Not only might it demotivate people to engage in physical activity, it may also influence the advice and intervention of physiotherapists. This study showed that the trackers are not valid for activities of daily living performed in this study. Considering this limitation, the trackers should only be used to measure steps during free living situations in which patients perform more walking activities. 


\section{Conclusion}

This study showed that the validity of 9 commercially available activity trackers is low measuring steps while individuals engage in activities of daily living among a diverse group of patients with various chronic diseases receiving physiotherapy. Frequent underestimation and a wide range of measurements were seen for step count during a protocol with activities of daily living compared to observed steps as gold standard.

\section{Acknowledgments}

We would like to thank all (consortium) partners: Hanze University of Applied Sciences, Hospital Nij Smellinghe, Fontys University of Applied Sciences School of Sport Studies, Maastricht University, Paramedisch Centrum Zuid, Fysiotherapie Schaesberg, Revalidatiecentrum Adelante, Ergotherapie Prakijk Zuid-Limburg, Royal Dutch Society for Physical Therapy and Huis voor de zorg. This project was funded by Stichting Innovatie Alliantie (2014-01-54P) and Brightlands Innovation Program Limburg Meet. The datasets used and/or analyzed during the current study are available from the corresponding author on reasonable request. 


\section{References}

1. Renders C, Boots T, Visser K, Roozen B. Smart Health Monitor: Onderzoek naar gebruik van apps, wearables en meters. 1st Edition. Hertogenbosch: Multiscope; 2016.

2. Crajé M, Hodselmans A, van Ittersum M, van Heeringen-de Groot D, Verhoef J, van der Schans C. Introduction-physical activity intervention.: Royal Dutch Society for Physical Therapy URL: https://www.fysionet-evidencebased.nl/images/pdfs/beweeginterventies/inleiding_bij_de_kngf-

standaarden_beweeginterventies.pdf

3. Verhagen SJM, Jongert MVA, Koers H, Toereppel K, Walhout R, Staal JB. KNGF Standaard beweeginterventie coronaire hartziekten. 2008. URL: https://www.fysionet-evidencebased.nl/images/ pdfs/beweeginterventies/standaard_bi_corn_hartziekten_2009_update3.pdf

4. Praet SFE, Van Uden C, Hartgens F, Savelberg HHCM, Toereppel K, de Bie RA. KNGF Standaard beweeginterventie diabetes mellitus type 2. 2009. URL: https://www.fysionet-evidencebased.nl/ images/pdfs/beweeginterventies/standaard_bi_dm2_2009.pdf

5. Troosters T, Jongert M, de Bie R, Toereppel K, de Gruijter EEMH. KNGF Standaard beweeginterventie chronisch obstructieve longziekten. URL: https://www.fysionet-evidencebased.nl/images/pdfs/ beweeginterventies/standaard_bi_copd_2009.pdf.

6. Koke AJA, Hilberdink S, Hilberdink WKHA, Reneman MF, Schoffelen T, vanHeeringen-de Groot D, et al. KNGF Standaard beweeginterventie chronische pijn. 2015. URL: https://www.fysionetevidencebased.nl/images/pdfs/beweeginterventies/standaard_bi_chronische_pijn.pdf.[

7. Stuiver M, Wittink H, Velthuis M, Kool N, Jongert W. KNGF Standaard beweeginterventie oncologie. URL: https://www.fysionet-evidencebased.nl/images/pdfs/beweeginterventies/standaard_bi_oncologie_ 2011.pdf.

8. Koke AJA, van den Ende CHM, Jansen MJ, Steultjens MPM, Veenhof C. KNGF Standaard beweeginterventie artrose. 2011. URL: https://www.fysionet-evidencebased.nl/images/pdfs/ beweeginterventies/standaard_bi_artrose_2011.pdf.

9. Ainsworth B, Cahalin L, Buman M, Ross R. The current state of physical activity assessment tools. Prog Cardiovasc Dis 2015;57(4):387-395.

10. Helmerhorst HJF, Brage S, Warren J, Besson H, Ekelund U. A systematic review of reliability and objective criterion-related validity of physical activity questionnaires. Int J Behav Nutr Phys Act 2012;9:103

11. Bassett D, John D. Use of pedometers and accelerometers in clinical populations: validity and reliability issues. Phys Ther Rev 2013 Jul 19;15(3):135-142.

12. Floegel TA, Florez-Pregonero A, Hekler EB, Buman MP. Validation of consumer-based hip and wrist activity monitors in older adults with varied ambulatory abilities. J Gerontol A Biol Sci Med Sci 2016 Jun 02.

13. Lahousse L, Verlinden VJA, van der Geest JN, Joos GF, Hofman A, Stricker BHC, et al. Gait patterns in COPD: the Rotterdam Study. Eur Respir J 2015 Jul;46(1):88-95

14. Mueller MJ, Minor SD, Sahrmann SA, Schaaf JA, Strube MJ. Differences in the gait characteristics of patients with diabetes and peripheral neuropathy compared with age-matched controls. Phys Ther 1994 Apr;74(4):299-309.

15. Kaufman KR, Hughes C, Morrey BF, Morrey M, An KN. Gait characteristics of patients with knee osteoarthritis. J Biomech 2001 Jul;34(7):907-915.]

16. Beevi FHA, Miranda J, Pedersen CF, Wagner S. An evaluation of commercial pedometers for monitoring slow walking speed populations. Telemed J E Health 2015 Oct 9;22:441-449.

17. Evenson KR, Goto MM, Furberg RD. Systematic review of the validity and reliability of consumerwearable activity trackers. Int J Behav Nutr Phys Act 2015;12(1):159.

18. Van Remoortel H, Giavedoni S, Raste Y, Burtin C, Louvaris Z, Gimeno-Santos E, PROactive consortium. Validity of activity monitors in health and chronic disease: a systematic review. Int J Behav Nutr Phys Act 2012 Jul 09;9:84.

19. Kooiman TJM, Dontje ML, Sprenger SR, Krijnen WP, van der Schans CP, de Groot M. Reliability and validity of ten consumer activity trackers. BMC Sports Sci Med Rehabil 2015;7:24. 
20. Ferguson $\mathrm{T}$, Rowlands AV, Olds $\mathrm{T}$, Maher $\mathrm{C}$. The validity of consumer-level, activity monitors in healthy adults worn in free-living conditions: a cross-sectional study. Int J Behav Nutr Phys Act 2015;12:42.

21. Kaewkannate K, Kim S. A comparison of wearable fitness devices. BMC Public Health 2016 May 24;16:433.

22. Mignault D, St-Onge M, Karelis AD, Allison DB, Rabasa-Lhoret R. Evaluation of the Portable HealthWear Armband: a device to measure total daily energy expenditure in free-living type 2 diabetic individuals. Diabetes Care 2005;28(1):225-227.

23. Arvidsson D, Slinde F, Nordenson A, Larsson S, Hulthén L. Validity of the ActiReg system in assessing energy requirement in chronic obstructive pulmonary disease patients. Clin Nutr 2006;25(1):68-74.

24. Langer D, Gosselink R, Sena R, Burtin C, Decramer M, Troosters T. Validation of two activity monitors in patients with COPD. Thorax 2009;64(7):641-642.

25. Vooijs M, Alpay LL, Snoeck-Stroband JB, Beerthuizen T, Siemonsma PC, Abbink JJ, et al. Validity and usability of low-cost accelerometers for internet-based self-monitoring of physical activity in patients with chronic obstructive pulmonary disease. Interact J Med Res 2014;3(4):e14.

26. Rabinovich RA, Louvaris Z, Raste Y, Langer D, Van Remoortel H, Giavedoni S, PROactive Consortium. Validity of physical activity monitors during daily life in patients with COPD. Eur Respir J 2013;42(5):12051215.

27. van der Weegen $S$, Essers $H$, Spreeuwenberg M, Verwey R, Tange $H$, de Witte $L$, et al. Concurrent validity of the MOX activity monitor compared to the ActiGraph GT3X. Telemed J E Health 2015;21(4):259-266.

28. Van Remoortel H, Raste Y, Louvaris Z, Giavedoni S, Burtin C, Langer D, PROactive Consortium. Validity of six activity monitors in chronic obstructive pulmonary disease: a comparison with indirect calorimetry. PLoS One 2012;7(6):e39198.

29. Cavalheri V, Donária L, Ferreira T, Finatti M, Camillo CA, Cipulo REM, et al. Energy expenditure during daily activities as measured by two motion sensors in patients with COPD. Respir Med 2011;105(6):922929.

30. Hill K, Dolmage TE, Woon L, Goldstein R, Brooks D. Measurement properties of the SenseWear armband in adults with chronic obstructive pulmonary disease. Thorax 2010;65(6):486-491.

31. Cox NS, Alison JA, Button BM, Wilson JW, Morton JM, Dowman LM, et al. Validation of a multi-sensor armband during free-living activity in adults with cystic fibrosis. J Cyst Fibros 2014;13(3):347-350.

32. Dijkstra B, Zijlstra W, Scherder E, Kamsma Y. Detection of walking periods and number of steps in older adults and patients with Parkinson's disease: accuracy of a pedometer and an accelerometry-based method. Age Ageing 2008 Jul;37(4):436-441.

33. Moy M, Garshick E, Matthess K, Lew R, Reilly J. Accuracy of uniaxial accelerometer in chronic obstructive pulmonary disease. J Rehabil Res Dev 2008;45(4):611-617.

34. Prieto-Centurion V, Bracken N, Norwick L, Zaidi F, Mutso AA, Morken V, et al. Can commercially available pedometers be used for physical activity monitoring in patients with COPD following exacerbations? Chronic Obstr Pulm Dis 2016;3(3):636-642.

35. Danilack VA, Okunbor O, Richardson CR, Teylan M, Moy ML. Performance of a pedometer to measure physical activity in a U.S. cohort with chronic obstructive pulmonary disease. J Rehabil Res Dev 2015;52(3):333-342.

36. Alharbi M, Bauman A, Neubeck L, Gallagher R. Validation of Fitbit-Flex as a measure of free-living physical activity in a community-based phase III cardiac rehabilitation population. Eur J Prev Cardiol 2016;23:1476-1485.

37. Boeselt T, Spielmanns M, Nell C, Storre JH, Windisch W, Magerhans L, et al. Validity and usability of physical activity monitoring in patients with chronic obstructive pulmonary disease (COPD). PLoS One 2016;11(6):e0157229.

38. Van Blarigan EL, Kenfield SA, Tantum L, Cadmus-Bertram LA, Carroll PR, Chan JM. The Fitbit One physical activity tracker in men with prostate cancer: validation study. JMIR Cancer 2017;3(1):e5.

39. Beekman E, Braun SM, Ummels D, van Vijven K, Moser A, Beurskens AJ. Validity, reliability and feasibility of commercially available activity trackers in physical therapy for people with a chronic disease: a study protocol of a mixed methods research. Pilot Feasibility Stud 2017;3:64.

40. Agusti A, Decramer M, Celli BR, Chen R, Criner G, Frith P, et al. Pocket guide to COPD diagnosis, management and prevention. 2017. URL: http://goldcopd.org/download/361/ 
41. Linn BS, Linn MW, Gurel L. Cumulative illness rating scale. J Am Geriatr Soc 1968 May;16(5):622-626.

42. de Groot V, Beckerman H, Lankhorst GJ, Bouter LM. How to measure comorbidity: a critical review of available methods. J Clin Epidemiol 2003;56(3):221-229.

43. Marshall AL, Smith BJ, Bauman AE, Kaur S. Reliability and validity of a brief physical activity assessment for use by family doctors. Br J Sports Med 2005 May;39(5):294.

44. Collen FM, Wade DT, Bradshaw CM. Mobility after stroke: reliability of measures of impairment and disability. Int Disabil Stud 1990;12(1):6-9.

45. Sant'Anna T, Escobar VC, Fontana AD, Camillo CA, Hernandes NA, Pitta F. Evaluation of a new motion sensor in patients with chronic obstructive pulmonary disease. Arch Phys Med Rehabil 2012 Dec;93(12):2319-2325.

46. Validation of the Active8 Activity Monitor: detection of body postures and movements.: Erasmus MC University Medical Center URL: https://www.activ8all.com/professional-use/medical-research-studieseducation/validation-of-activ8/

47. de Vet HCW, Terwee CB, Knol DL, Bouter LM. When to use agreement versus reliability measures. J Clin Epidemiol 2006;59(10):1033-1039.

48. De Vet H, Terwee C, Mokkink L, Knol D. Measurement in Medicine: A Practical Guide. Cambridge: Cambridge University Press; 2011.

49. Bland JM, Altman DG. Statistical methods for assessing agreement between two methods of clinical measurement. Lancet 1986;1(8476):307-310.

50. Martin JB, Krč KM, Mitchell EA, Eng JJ, Noble JW. Pedometer accuracy in slow walking older adults. Int J Ther Rehabil 2012;19(7):387-393.

51. Cyarto EV, Myers AM, Tudor-Locke C. Pedometer accuracy in nursing home and community-dwelling older adults. Med Sci Sports Exerc 2004;36(2):205-209.

52. Nelson MB, Kaminsky LA, Dickin DC, Montoye AHK. Validity of consumer-based physical activity monitors for specific activity types. Med Sci Sports Exerc 2016;48(8):1619-1628.

53. 15 best pedometer apps for walking and running.: Stepcounter URL: https://www. stepcounterpedometer.com/best-pedometer-apps/ [accessed 2017-07-21]

54. Bumgardener $W$. The 10 best pedometers for counting your steps. 2016. URL: https://www.verywell.com/top-pedometers-3435756.

55. Martin J. The best fitness trackers for 2017.: Tech Advisor; 2017. URL: http://www. techadvisor.co.uk/test-centre/wearable-tech/best-fitness-trackers-2017-3498368/ 
3 


\section{Chapter 3}

Patients' experiences with commercially available activity trackers embedded in physiotherapy treatment: a qualitative study

Darcy Ummels, Emmylou Beekman, Albine Moser, Susy M. Braun \& Anna J. Beurskens Disability and Rehabilitation 2019 Apr 11:1-9 


\section{Abstract}

\section{Aim}

The aim of this study was to describe the experience with commercially available activity trackers embedded in the physiotherapy treatment of patients with a chronic disease.

\section{Methods}

In a qualitative study, 29 participants with a chronic disease participated. They wore an activity tracker for two to eight weeks. Data were collected using 23 interviews and discussion with 6 participants. A framework analysis was used to analyze the data.

\section{Results}

The framework analysis resulted in seven categories: purchase, instruction, characteristics, correct functioning, sharing data, privacy, use, and interest in feedback. The standard goal of the activity trackers was experienced as too high, however the tracker still motivated them to be more active. Participants would have liked more guidance from their physiotherapists because they experienced the trackers as complex. Participants experienced some technical failures, are willing to share data with their physiotherapist and, want to spend a maximum of $€ 50,-$.

\section{Conclusion}

The developed framework gives insight into all important concepts from the experiences reported by patients with a chronic disease and can be used to guide further research and practice. Patients with a chronic disease were positive regarding activity trackers in general. When embedded in physiotherapy, more attention should be paid to the integration in treatment. 


\section{Introduction}

Activity trackers are increasing in popularity, with the top five brands combined selling 102.4 million activity trackers worldwide in 2016. ${ }^{1}$ These activity trackers are primarily targeted at a healthy and athletic population, but they might have potential for other specific groups such as those in healthcare. In 2016 almost 9 million people in the Dutch population (52\%) suffered from one or more chronic diseases, with neck- and back pain, osteoarthritis and Diabetes Mellitus in the top three. ${ }^{2}$ Over $90 \%$ of the inhabitants who are 70 years or older have one or more chronic diseases, the prevalence in inhabitants younger than 40 years is already $35 \%{ }^{2}$ There is a positive relationship between physical activity and reduced premature death and the prevention of chronic diseases. ${ }^{3-5}$ For example, sufficient physical activity can reduce pain for people with osteoarthritis, can effectively control fasting and post-walk blood sugar levels in patients with Diabetes Mellitus and can reduce the risk of emergency admission in patients with chronic lung diseases. ${ }^{3}$ An adequate level of physical activity is one of the main points stressed in daily clinical practice, especially in physiotherapy, and recommended in evidence-based professional guidelines. ${ }^{6,7}$ Therefore, people with a chronic disease are a target group par excellence for additional support through monitoring and objectively measuring physical activity in daily life.

Physiotherapists provide professional support with tailored advice on lifestyle changes ${ }^{6}$ based on physical activity level and activity time distribution per day. They use questionnaires and diaries to measure the physical activity levels of their participants. However, questionnaires and diaries are time-consuming, have limited reliability and validity and depend on the patient's memory. ${ }^{8,9}$ A promising alternative to overcome these limitations is activity trackers. They can provide an objective measurement of the person's physical activity level, give insight into the distribution of physical activity levels during the day, and may motivate people to enhance their daily activity level. ${ }^{10}$ The use of activity trackers in healthcare can aid in monitoring treatment results in the patient's daily life, increasing self-management, saving time and money, while addressing the actual setting where the lifestyle change should be achieved.

A good quality measurement device is important for implementation purposes. Several publications can be found regarding the clinimetric properties, validity and reliability of activity trackers. ${ }^{11-14}$ Activity trackers have been shown to be valid during walking and running, however in daily living, activity trackers have a lower validity in people with a chronic disease. $^{11-14}$

Another important property concerns feasibility, i.e., experiences with activity trackers in healthcare. In this context, feasibility is understood as an umbrella concept, including 
experiences regarding user-friendliness as well as acceptability and usefulness in daily practice.

Feasibility aspects such as user-friendliness and sustained use of activity trackers have been studied in healthy adults ${ }^{15-22}$ and the elderly. ${ }^{23-28}$ From these studies it appears that in general, healthy adults and the elderly are open and positive towards the use of activity trackers. However, most studies argue that more attention should be paid to user experiences and lack of technical skills of the user. This may also lead to a more sustained use of the activity trackers in daily life.

By contrast, little is known about the experiences with activity trackers embedded in the healthcare of people with chronic diseases. Only two studies have described several important experiences and needs of people with a chronic disease, such as wearing comfort, feedback, validity, reliability and the added value of feedback. ${ }^{29,30}$ However, they did not focus on the experiences with the use of the activity trackers in healthcare. To incorporate activity trackers in healthcare, like physiotherapy treatment, insight into feasibility from the perspective of people with a chronic disease who use activity trackers in a healthcare setting must be generated.

Therefore, the aim of this study was to describe how patients with a chronic disease experienced the use of commercially available activity trackers embedded in physiotherapy.

\section{Methods}

A qualitative design was used, based on the tenets of qualitative inquiry ${ }^{31}$, using interviews and a focus group discussion to collect data. A framework study was used to analyze the data.

\section{Participants}

Participants were people with chronic diseases under treatment by physiotherapist. The inclusion criteria were diagnosed with at least one of the following chronic diseases: cardiovascular disease, chronic obstructive pulmonary disease (COPD), diabetes mellitus (DM), chronic pain, cancer or osteoarthritis. Exclusion criteria were: insufficient understanding of the Dutch language, use of a walking aid, or an asymmetrical gait. Participants were recruited via purposive sampling ${ }^{32}$ based on their chronic disease from two physiotherapy practices (Fysiotherapie Schaesberg and ParaMedisch Centrum Zuid) and a rehabilitation center (Adelante Zorggroep) in The Netherlands. All participants provided written informed consent after receiving verbal and written information about 
the research. This study was approved by the local ethics board (METC Atrium-OrbisZuyd; 15-N-48).

\section{Activity trackers}

Eight activity trackers were selected based on characteristics such as wearing position, type of activity tracker and trackable activities, and on selection criteria required by the physiotherapists (costs less than $€ 150$, no monthly subscription, real-time feedback from the tracker to the user, measures number of steps, and no chest strap to measure heart rate). The following eight activity trackers were selected: Activ8, Digi-Walker CW-700, Fitbit Flex, Lumo Back, Moves, Fitbit One, UP24, and the Walking Style X (Table 3.1). ${ }^{33}$ Participants were provided with an activity tracker by their physiotherapist and asked to wear it for at least a week. Intentionally, physiotherapists received no specific instructions regarding to the way activity trackers should be distributed. The choice was made in consultation with the participant (shared decision-making) or was made by the physiotherapist solely. At their own request, participants could wear the activity tracker longer and could also use a second activity tracker. The physiotherapists had two training sessions: one about the practical use of the activity tracker (e.g., how to install them) and one about the integration of activity trackers in their therapy. Physiotherapists did not receive any further instructions about how to use the activity trackers in their treatments, to simulate 'real life' as much as possible.

Table 3.1 Eight selected commercially available activity trackers used in this study.

\begin{tabular}{lllll}
\hline Activity tracker & Manufacturer & Type & $\begin{array}{l}\text { Wearing } \\
\text { position }\end{array}$ & $\begin{array}{l}\text { Outcome } \\
\text { variables }\end{array}$ \\
\hline Activ8 & Remedy Ltd & Accelerometer & Trouser pocket & A,B,C \\
Digi-Walker CW-700 & Yamax Coorporation & Pedometer & Wrist & A,C \\
Flex & Fitbit Inc. & Accelerometer & Wrist & A,C \\
Lumo Back & Lumo BodyTech, Inc. & Accelerometer & Lower back & A,B,C,D \\
Moves & ProtoGeo & App & Trouser pocket A,C \\
One & Fitbit Inc. & Accelerometer & Belt & A,C \\
UP24 & Jawbone & Accelerometer & Wrist & A,C \\
Walking StyleX & Omron Healthcare Europe B.V. Pedometer & Belt & A,C \\
\hline
\end{tabular}

A: number of steps; B: time spent lying, sitting, standing, walking, running and cycling; C: active minutes; D: number of sit to stand transition.

\section{Data collection}

For socio-demographic purposes, the participants' general characteristics were collected: gender, age, diagnosed chronic disease, and the highest level of education. Specific disease characteristics were collected. In the case of COPD, this was the Global 
Initiative for Chronic Obstructive Lung Disease (GOLD) stage ${ }^{34}$, for osteoarthritis its location (lower extremity, upper extremity, cervical or lower spine), and in cancer patients the treatment phase (curative/palliative). Two questionnaires were used: the Physical Activity Questionnaire (PAQ) and the Cumulative Illness Rating Scale (CIRS). The PAQ was used to indicate the daily physical activity of the participants ${ }^{35}$, and the CIRS was used to calculate the severity and number of comorbidities. ${ }^{36,37}$

Participants were asked to participate in an individual interview or focus group discussion. A semi-structured interview guide for the individual interviews was developed based on the five steps of Kallio et al. ${ }^{38}$ In step 1, the appropriateness of the semi-structured setup was verified as a rigorous data collection method in relation to the research question of this study. In step 2, previous knowledge was retrieved from the literature and by consulting experts (two participant representatives, the research team and, two mHealth experts) to understand the phenomenon of feasibility, i.e., experiences with activity trackers in healthcare. In step 3, the preliminary interview guide was formulated. In step 4, the interview guide was tested by means of internal testing: evaluation within the research team, expert assessment with two participant representatives, and field testing with the first two research participants. Adaptations were made, and in step 5, the complete semi-structured interview guide was finalized. The interview guide is provided in Supplement File S3.1. The questioning route used for the focus group discussions was developed according to Krueger et al. ${ }^{39}$ The questioning route for the focus group discussion was based on that of the individual interviews to confirm data saturation.

The interviewers were a physiotherapist (EB) or a human movement scientist (KVV). The interview questions were openended to encourage the participants to talk about their experiences. If needed, the interviewer asked follow-up questions to gain more insight. Individual interviews were performed at a location convenient for the participant (either the participant's home or the physiotherapy practice). Each interview was audio recorded and lasted 30-60 min; the interviewer also took field notes. The focus group discussion took place at the research institute (Zuyd University). The focus group discussion was audio recorded and lasted for 60-90 min. There was one interviewer (EB), two researchers took field notes $(\mathrm{KvV}, \mathrm{AB})$ and two participant representatives were present.

Participants were included until no new information was collected (data saturation) on all topics. This was established by regular agreement sessions within the research team. Once data saturation had been reached, one focus group discussion was scheduled, which served to confirm the results. 


\section{Data analysis}

The data were analyzed according to the Framework Method. ${ }^{40}$ In stage 1 , all audio recordings were transcribed verbatim by two researchers (DU, KT) and primary identifiers were de-identified. In stage 2 , the entire transcripts were read and, if needed for the context of the text passage, the audio recordings were reviewed. In stage 3 , paraphrases or labels (a code) were applied to relevant text fragments. Deductive and inductive content analysis was used. ${ }^{41}$ Deductive content analysis was used for the majority of codes predefined according to empirical information. ${ }^{16-19,22-24,29,30,42-47}$ Inductive coding was applied when the text passages did not fit a predefined code but were considered to be relevant. An 'other' code was defined to include such data. During the analysis, two subcategories were added to the framework: choice of activity tracker and discussing results with a physiotherapist. In stage 4, the first two interviews were coded by both researchers (DU, KT). An alignment session was held with the two researchers and one other independent researcher (EB) to fine-tune the coding. Differences in interpretation were solved by a dialog between DU and KT to reach consensus, because the aim was the find a suitable interpretation grounded in the original quote. The codes were grouped together into categories and subcategories by the research team, using a tree diagram. Expert assessment of this working analytical framework took place in four iterative expert meetings with two participant representatives, the research team, and two mHealth experts. In stage 5, the analytical framework was applied by indexing subsequent transcripts using the existing categories and codes (see Supplement File S3.2). In stage 6, the data were entered into the framework matrix from a spreadsheet including a summary and a reference to illustrative quotations. In addition, an analytical tabulation was performed. Finally, in stage 7, the data were interpreted and presented in a descriptive way using NVivo (version10).

Descriptive statistics of the participants' characteristics were presented. Means ( \pm standard deviation) or medians (range) (depending on the data distribution) were given. If data were missing, pairwise deletion was applied.

\section{Trustworthiness}

To ensure the quality and trustworthiness of this study, credibility and transferability were checked in several ways. ${ }^{31}$ Credibility was examined by method, investigator and data triangulation. Method triangulation involved multiple methods of data collection (interviews and focus group discussions); investigator triangulation was achieved by having all authors reflect on the design, data collection and analysis process during this study; and data triangulation used different sources of the same information (multiple 
interviewees and participants in focus group discussions). Transferability was examined through a thick description of our study population and the study process. ${ }^{31}$

\section{Results}

The framework analysis resulted in eight categories: 1) purchase of the activity trackers, 2) instruction, 3) characteristics of the activity tracker, 4) correct functioning, 5) sharing data, 6) privacy, 7) use of the activity tracker, and 8) interest in feedback. The number of quotes per (sub)category is presented in Supplement File S3.2.

\section{Participants}

A total of 23 participants were interviewed individually, and six participants participated in the focus group discussion (Table 3.2); all wore the activity tracker for between two and eight weeks. The Activ8 was the most used activity tracker $(n=6)$, followed by the Fitbit Flex ( $n=5)$, Fitbit One ( $n=5)$, Digiwalker CW-700 ( $n=4)$, UP $24(n=4)$, Walking Style X $(n=4)$, the Moves app $(n=2)$ and, the Lumoback $(n=1)$. Four participants used two different activity trackers, two participants tested three different activity trackers, and one participant tested four different activity trackers.

Table 3.2 Demographic and health characteristics of included participants.

\begin{tabular}{|c|c|}
\hline Characteristics & Participants $(n=29)$ \\
\hline Gender, $\mathrm{n}$ male (\%) & $7(27)$ \\
\hline Age (years), median (range) & $55(22-78)$ \\
\hline \multicolumn{2}{|l|}{ Education, $\mathrm{n}(\%)$} \\
\hline Secondary Education & $6(26)$ \\
\hline College & $9(39)$ \\
\hline University & $8(35)$ \\
\hline \multicolumn{2}{|l|}{ Diagnosed disease, n (\%) } \\
\hline Cardiovascular Disease & $1(4)$ \\
\hline COPD & $2(8)$ \\
\hline Diabetes Mellitus & $1(4)$ \\
\hline Cancer & $5(20)$ \\
\hline Osteoarthritis & $4(16)$ \\
\hline Chronic Pain & $10(40)$ \\
\hline Combination & $2(8)$ \\
\hline Comorbidity (CIRS 0-52), median (range) & $5(0-10)$ \\
\hline \multicolumn{2}{|l|}{ Physical activity* } \\
\hline Sufficiently active, n (\%) & $14(59)$ \\
\hline Insufficiently active, $\mathrm{n}(\%)$ & $10(41)$ \\
\hline
\end{tabular}


Chronic pain, osteoarthritis, and cancer were the most prevalent conditions, and $8 \%$ of the participants had multimorbidity. Of the participants with COPD, one patient had been diagnosed with stage II COPD, and one patient didn't know his GOLD stadium. Of the participants with cancer, $50 \%$ had had a curative treatment and $50 \%$ a palliative treatment; this variable was missing for one participant. Of the participants with osteoarthritis, the most affected joints were the upper extremity (41\%), spine (36\%) and lower extremity (23\%).

\section{Purchase of the activity trackers}

Most of the participants were unfamiliar with activity trackers and had no idea about their average cost. Some of the participants had heard that activity trackers were expensive. When asked about how much they were willing to pay, participants said they would be willing to spend between 20-50 euros but that there should be no other costs, e.g., subscription fees. Some participants wanted to buy an activity tracker but refrained due to the perceived high costs.

"I would spend 20-30 euros if I am sure the activity tracker works. But I can get one for 5 euros of which I am not sure if it works." Female, 28 years, chronic pain

"We subscribed somewhere so we could get a discount on a Fitbit. My husband asked if he should buy one for me. But I think it is way too expensive" Female, 57 years, cancer

Some looked for reimbursement options and consulted their insurance company. If healthcare insurance companies offered some form of compensation, then participants would consider buying an activity tracker at the average retail price.

"I already called the health insurance company to see if they reimburse it [activity tracker] but they don't." Female, 65 years, Diabetes Mellitus and cardiovascular disease

One other difficulty in purchasing an activity tracker was the lack of information about them. The amount of effort needed to find information hindered them from buying one. According to the participants, there was no clear information available on the internet. Participants wanted an aid that compared several activity trackers. They suggested one that would specify the characteristics, brand, advantages and disadvantages of a small number of activity trackers and where they could be obtained. Some participants also suggested linking this to their physiotherapist's website or providing information in the physiotherapist practice. 
"Well a scheme, with the possibilities and for example a picture and where you can buy it [activity tracker]. Or which brand, what the advantages and disadvantages of some are. Or the complexity." Female, 46 years, chronic pain

Participants found that activity trackers did not function on Apple products (MacBook, iPad) and that some older computers can't run the activity tracker software. They experienced this as disappointing. However, they would not buy another computer or smartphone so they could use their activity tracker.

\section{Instruction and use}

Older participants said that if they had received more information from their physiotherapists about using the activity tracker, they could have used them independently. Some participants consulted the physiotherapist for additional information. Almost all had to ask their partner, children, or physiotherapist for extra help.

"I didn't understand how to do it, so I called my physiotherapist again and we went through it together one more time, after that it went fine." Male, 66 years, Diabetes Mellitus and cardiovascular disease

"I installed the activity tracker together with my husband. I have to admit, I don't have that much technical skills, but together we managed to do it." Female, 61 years, chronic pain

Most participants mentioned that their physiotherapist installed the activity tracker for them. Some participants had asked their physiotherapist to read out their data. Others claimed that by embedding activity trackers in the care process, the physiotherapist needed to focus on several aspects in daily clinical practice: a thorough explanation of the activity tracker, advice on the suitable moment(s) of checking individual data on the trackers and help with the translation of the activity tracker data into a conclusion for the patient on how he is doing ('data interpretation').

"In the light of embedding in healthcare, the physiotherapist should pay more attention to explaining the activity tracker, and the meaning of it, but he should also help with the interpretation of the results." Male, 56 years, cardiovascular disease

With some activity trackers, an instruction guide was included, yet experiences with these guides varied between participants. Some participants found the instructions very clear, while others said they were too vague. Many participants would like a clear stepby-step manual. One major issue was that the guides were written in English instead of 
the participants' mother tongue (Dutch). But even if the manual was written in Dutch, the activity tracker itself, the apps and web portals were still in English instead of Dutch, which still made them hard to understand and operate.

"Well, make it clear, add a Dutch manual with it, how the install it [activity tracker] and stuff like that." Female, 57 years, cancer

Most participants felt that their technical abilities were insufficient. The most commonly expressed explanation was their age. Participants were reluctant to try different settings and buttons on the activity tracker as they might do something wrong and lose the data.

"No, well, I always think, don't touch it, you never know what you can do wrong. My son works in IT, and he always says: Mom, you can't do that, you can always get it [data] back." Female, 66 years, cardiovascular disease and cancer

Some wrote down the data on paper and brought it to the consultation with the physiotherapist because they feared they would lose data and damage the activity tracker.

"I think if I do this, I lose everything. Maybe it is very easy if you would do it. But I am afraid that I would do something wrong. I prefer to watch and write down the time and date and number of steps. I take the note with me and say: This is what I have done. I am afraid that I would break something again, and I don't want that." Female, 70 years, chronic pain

\section{Characteristics of the activity tracker}

Participants expressed that activity trackers should be as easy to use as possible (e.g., one button). It was hard for them to navigate and find their data in the corresponding app or computer dashboard. Because of the technical and procedural complexity of the activity tracker, they did not try to understand it fully. Some felt if they had tried harder, they would have understood the activity tracker better.

"I did find the number of steps, but you had to push buttons to get more information, so you really needed to search. How do I find what I want and what does it all mean? I just didn't think it was practical." Male, 41 years, chronic pain

This complexity demotivated them to search for the information on the app or dashboard. 
"Especially number of steps and the walked distance, I had to search for the rest. You really had to look how to get more information. Sometimes I don't feel like searching for it." Male, 41 years, chronic pain

The activity trackers measure several variables, and most participants were interested in their number of steps, calories, sleep pattern and walked distance. When specifically asked, some participants would have liked to have variables related to swimming, cycling, and a heart rate monitor. The option to register their food and liquid intake was hardly used.

"It should register all kind of movement, such as cycling and walking, and I would like to measure my heartrate and blood pressure as well." Female, 60, chronic pain

Most participants used the interface of the activity tracker itself to check their data and to receive feedback, provided that the text on the activity tracker was legible. Participants checked their data regularly, varying from once a day to several times during the day. Some participants would have liked an overview of their physical activity during the week, in the form of a graph. Participants appreciated the visual stimulant.

"I saw that I was above the line, which was enough for me. But it would be nice, not every day, but once a week for example that you can see the difference with yesterday in a simple way." Female, 66 years, cardiovascular disease and cancer

Most participants focused on the number of steps as a daily goal, while some used the number of calories, but the majority took the standard goal of the activity tracker, the 10,000 steps. For many, this standard goal was too high, though almost no one altered it. The most common personal health goal of the participants was to walk more or lose weight. Some participants mentioned they did not have a specific goal but wanted to gain insight into their physical activity or into the relationship between their physical activity and their chronic disease or rehabilitation process.

"When I used to come home from the clinic where I was treated, I couldn't do anything. I could walk three steps. That has a certain progression. I would have liked to oversee this whole process. What was I able to do yesterday and what am I able to do now?" Male, 62 years, osteoarthritis

The preferred place to wear the activity tracker was a trouser pocket attached with a clip, though several women preferred wearing the activity tracker attached to their bra.

"Yes, in my trouser pocket, always in my trouser pocket, like now. It doesn't bother me at all, I just have to remember it when I change my trousers." Male, 62 years, osteoarthritis 
Most participants reported that the activity tracker should be discrete, easy to attach without assistance, and comfortable to wear. Sleeping with the activity tracker was uncomfortable for most participants.

"I liked the Fitbit One better, the small thing, that is because I don't like it if it [activity tracker] is visible" Female, 47 years, chronic pain

Some expressed concern that the activity trackers were not waterproof, or could easily be lost.

"I thought it was a pity it [activity tracker] wasn't waterproof. We still have to wash windows and do our dishes and stuff like that." Female, 60 years, chronic pain

The battery of the activity tracker lasted longer than expected. Participants appreciated the average lifetime of 3-4 weeks. They stated that there was a chance they would forget to recharge the activity tracker regularly.

\section{Correct functioning}

The experiences with the validity and reliability of the activity trackers varied. Small movements such as household activities were sometimes not measured. In some instances the activity tracker measured activities that were not actually performed by the participants.

"It [activity tracker] registered cycling but I never cycle and yet it popped up, for example, 20 minutes or something like that and I figured maybe I had made some kind of movement which is registered as cycling?" Female, 30 years, chronic pain

Participants noticed a lack of validity and reliability more often with activity trackers worn around the wrist. Sometimes, participants had technical problems such as trouble logging-in or synchronizing with the activity tracker.

"It could take up to a half hour before the activity tracker made a connection" Female, 47 years, chronic pain

\section{Sharing data and privacy}

Participants were positive about sharing data with their family or friends. This gave them a confirmation of their physical activity level and simultaneously challenged them to be more physically active. 
"I used to do this with some friends from the North (of the Netherlands) and from here. I showed them how much I walked." Female, 74, osteoarthritis

None expressed reticence about sharing their data with healthcare professionals, and none wished to share their data with companies, including the manufacturer of the activity tracker.

"I don't think the data are privacy sensitive. If he [physiotherapist] can help me by having insight in the data, I can benefit from that of course." Female, 61 years, chronic pain

\section{Use of the activity tracker}

Participants used their activity tracker, but over time some of them forgot to wear or check it. The majority of participants did not discuss their physical activity data with their physiotherapist, though when asked, some admitted they would have liked to do so. Only a few discussed their results with their physiotherapist and created new activity goals together. The data were verbally discussed without using the activity tracker's interface. None of the participants wanted to discuss the data during their treatment session, since they valued the treatment delivered by the physiotherapist more than any substitution of treatment by the data of the activity tracker. Another reason was that some of the participants received group therapy and did not want to request extra time from their physiotherapist.

"If the physiotherapist should read out the data and explain them, that would take too much time away from my treatment" Male, 63 years, osteoarthritis

Only when asked, participants thought it would be motivating to discuss the data with their physiotherapist. As this wasn't the case, most did not recognize the added value of the activity tracker.

"I: Would it [activity tracker] be an added value to your therapy?" "P: Yes, I don't know what could be discussed. Well, it isn't right or wrong. So maybe some kind of guideline, I think you have moved too little or something like that? That is always nice to hear. Whether you do something with it depends on yourself of course. I still don't know what average is, that is a nice thing what I would like to know."

Female, 44 years, chronic pain

Participants found it demotivating to deal with unrealistically high goals. Participants would have liked to create an individual goal together with their physiotherapist. Some 
became too active for their condition, which led to physical complaints. A few used the goals as a 'brake' to limit their physical activity.

"Well it [step count goal] is not achievable. And you start to think, "I won't make it anyway', so it should be achievable goals" Female, 47 years, chronic pain

\section{Interest in feedback}

All participants, except one, perceived the feedback of the activity tracker as positive and pleasant. One perceived the feedback as negative, due to a decline in his health. They used the feedback as a motivator to reach their goals; almost all stated that they went for an extra walk or climbed some more stairs to achieve their target. However, it was demotivating when participants noticed that the activity trackers did not measure certain activities (cycling, walking stairs).

"I started walking through the gallery and the living room just to... It really has been a challenge, but at some point it became obsessive. If I open that thing [activity tracker] at 22.00 and I thought, 'Oh no, these are not a thousand steps, I should do a little more'. So I ran at the end of the day through my home just to make more steps." Female, 73 years, cancer

Participants also reported that they became more aware of their physical activity level in general.

"I think when it [number of steps] is visible, it will motivate you to complete the task." Male, 66 years, cancer

The visual stimulant of achievement, for instance a growing flower or a smiley, was well received by almost all participants. It motivated them to gain a reward from the activity tracker (e.g., fully grown flower). Some appreciated that feedback was an objective measurement.

"I think it [activity tracker] stimulates you to walk more because it is visible. If you don't have it [activity tracker], then you don't of course." Female, 75 years, osteoarthritis

\section{Discussion}

The aim of this study was to describe how patients with a chronic disease experienced the use of commercially available activity trackers embedded in physiotherapy 
treatment in order to increase successful implementation in future care. The participants used an activity tracker for at least one week and were then interviewed about their experiences. In general, they experienced the use of the activity trackers as positive and enjoyed using them. The activity trackers motivated them to increase their physical activity levels and reach their daily goals, and, they became aware of their physical activity. However, they experienced certain limitations, such as the complexity of the activity tracker, doubts about its validity and reliability, the lack of clear instructions for using the activity tracker by the physiotherapist, and high standard goals set by the activity tracker. The majority of the participants did not discuss their activity tracker data with their physiotherapists, as the treatment delivered by the physiotherapist was valued more than the data of the activity tracker.

The results of the interviews are in line with previous studies ${ }^{29,30}$ in which the acceptance and usefulness of activity trackers in their daily life for people with a chronic disease was examined. Mercer et al. concluded that activity trackers were perceived as acceptable and useful, but the participants needed support in setting up the device and interpreting the data. ${ }^{29}$ Rosenberg et al. also concluded that men with prostate cancer perceived activity trackers as acceptable but found several barriers to their use such as problems with syncing the activity trackers and data inaccuracies. ${ }^{30}$ Activity trackers should be as straightforward as possible, with personal demonstrations and written manuals provided. Mercer et al. and Rosenberg et al. both suggested that usability could be improved by having more compatible computers and smartphones, comprehensive paper manuals, and apps that interpret the user data. Studies among the elderly and adults $^{15-28}$ showed considerable similarities with the results of this study and those of Mercer et al. and Rosenberg et al. Older people and adults accepted the activity tracker, found them stimulating, increased awareness, and experienced them as useful. However, especially elderly stated they would prefer an activity tracker which is easier to use and adapted to their needs and skills. Adults prefer an activity tracker adapted to their routines and needs, and like to have a more accurate (i.e., reliable and valid) activity tracker.

In this study, a framework analysis was used to analyze the data, resulting in a framework with seven categories: 1) purchase of the activity trackers, 2) instruction, 3) characteristics of the activity tracker, 4) correct functioning, 5) sharing data and privacy, 6) use of the activity tracker, and 7) interest in feedback. Each category had several subcategories. The two themes, all categories and sub-categories, except for four subcategories, of the framework were consistent with the literature used. Two new subcategories were added during the analysis of the interviews: choice of the activity tracker and discussing results with a physiotherapist. A final framework was produced which includes all of the important concepts from the experiences reported by people 
with a chronic disease after using activity trackers and can guide the use in further research and practice.

\section{Limitations and strengths}

This study has certain strengths and limitations. The activity trackers used were the most up-to-date at the time of the study. However, some manufacturers stopped producing these specific kinds of tracker or stopped producing activity trackers entirely, while several new activity tracker brands and updated versions have been released with new functions. To the authors' knowledge, though, none of these activity trackers are designed for people with a chronic disease or even the healthcare sector in general. However, the concepts of the framework are general and still applicable to updated versions and new brands.

The length of time the participants used the activity tracker varied. Every participant used the activity tracker for at least one week, and they were free to use the activity tracker for a longer period of time if they desired. This might have influenced their experience since they would become more familiar with the tracker. Some participants used more than one activity tracker, which may also have biased their experience since they might have compared the activity trackers during the interview. This might also have intensified the experiences, resulting in enriched data in this study.

One strength of this study is the use of the Framework Method, which has been used in research for over 25 years. ${ }^{41}$ Some of its strong points are: data can be easily summarized, the structure is visually easy to interpret, it can be used with inductive and deductive analysis, the systematic procedure is easy to follow and has a clear audit trail. $^{41}$

Another strength of this study is the implementation of the activity tracker in physiotherapy treatment. The participants were free to use the activity tracker any way they liked, but almost none used it in their physiotherapy treatment. One explanation was that they valued the treatment for their physical complaints more than discussing their physical activity data. The participating physiotherapists received two training sessions and no further instructions about the use of the activity tracker in their daily practice. This information was probably too limited and more training including show cases is needed. It might be possible that physiotherapists therefore had too limited knowledge how to imbed the use of activity trackers in their treatment. The participants may also have not seen the added value of an activity tracker during their therapy, due to lack of guidance from their physiotherapist. If embedded correctly, activity trackers can potentially contribute in a positive way to the physical behavior of a patient. 


\section{Clinical relevance}

Activity trackers can potentially be of added value to physiotherapy, but the feasibility of the activity tracker must be optimal to ensure implementation in physiotherapy treatment. The findings in this study are novel, physiotherapists should be aware of several factors which might compromise the use of an activity tracker during physical therapy. First, activity trackers should be easy to use in daily life and during treatment. An activity tracker should match with the needs and technical skills of the patient, but currently patients experience them as too complex. Participants who have limited technical skills need regular guidance from their physiotherapist besides help with interpretation of activity tracker data for all patients.

At this moment, most of the participants did not see the added value of an activity tracker, since they didn't discuss the activity tracker data during their therapy and valued the physical treatment more. However, when asked, participants could see the potential added value of an activity tracker. Therefore, if a physiotherapist wants to use an activity tracker in therapy, they should use it in a meaningful way to support the physical treatment. An activity tracker can be a good measurement- and motivation tool in physiotherapy treatment since participants found them motivating to be more active and to reach their daily activity goals. However, some activity trackers are not yet valid and reliable during activities of daily living. This should be kept in mind by both the physiotherapists and patient, since this might demotivate the patient and influence the advice of the physiotherapists. Furthermore, due to the perceived high costs, most participants are not willing to buy an activity tracker themselves. A physiotherapist should keep in mind that therefore the use of an activity trackers might not be affordable for every patient depending on their finical situation and willingness to buy an activity tracker.

\section{Conclusion}

Patients with a chronic disease were positive regarding activity trackers in general. However they require an activity tracker adapted to their needs and skills. The developed framework gives insight in all important concepts from the experiences reported by people with a chronic disease and can be used to guide further research and practice. When embedded in physiotherapy, however, more attention should be payed to the integration in treatment. 


\section{Acknowledgements}

The authors would like to thank all (consortium)partners: Hanze University of Applied Sciences, Hospital Nij Smellinghe, Fontys University of Applied Sciences School of Sport Studies, Maastricht University, Paramedisch Centrum Zuid, Fysiotherapie Schaesberg, Revalidatiecentrum Adelante, Royal Dutch Society for Physical Therapy and, Burgerkracht Limburg. The authors also would like to thank Kim van Vijven and Kyra Theunissen for their contributions (data collection and analysis) during this study. 


\section{References}

1. International Data Coroporation [Internet]. Wearables Aren't Dead, They're Just Shifting Focus as the Market Grows $16.9 \%$ in the Fourth Quarter, According to IDC. Framingham: International Data Coroporation; 2017 [cited 2017 Nov 17]. Available from: https://www.idc.com/getdoc. jsp?containerld=prUS42342317

2. National Institue for Public Health and Enviroment [Internet]. Chronische ziekten en multimorbiditeit!Cijfers \& Context!Huidige situatie. Bilthoven: National Institue for Public Health and Enviroment; 2017 [cited 2018 Feb 7]. Available from: https://www.volksgezondheidenzorg.info/onderwerp/chronische-ziekten-en-multimorbiditeit/cijferscontext/huidige-situatie

3. C3 Collaborating for Health. The benefits of regular walking for health, well-being and the environment. London: C3 Collaborating for Health; 2012.

4. Warburton DE, Nicol CW, Bredin SS. Health benefits of physical activity: a systematic review of current systematic reviews. Curr Opin Cardiol. 2006;174:801-809.

5. Booth FW, Roberts CK, Laye MJ. Lack of exercise is a major cause of chronic diseases. Compr Physiol. 2012;2:1143-1211.

6. Fysionet-evidencebased [Internet]. Introduction physical activity intervention. Amersfoort: Royal Dutch Society for Physical Therapy, 2013 [cited 2017 Jun 7]. Available from: https://www.fysionetevidencebased.nl/images/pdfs/beweeginterventies/inleiding_bij_de_kngf-standaarden_beweeginterventies.pdf

7. World Health Orginsation. Global recommendations on physical activity for health. Switzerland: World Health Orginsation; 2010.

8. Ainsworth B, Cahalin L, Buman M, et al. The current state of physical activity assessment tools. Prog Cardiovasc Dis. 2015;57:387-395.

9. Helmerhorst HJ, Brage S, Warren J, et al. A systematic review of reliability and objective criterion-related validity of physical activity questionnaires. Int J Behav Nutr Phys Act. 2012;9:103.

10. Sullivan AN, Lachman ME. Behavior change with fitness technology in sedentary adults: a review of the evidence for increasing physical activity. Front Public Health. 2016;4:289.

11. Ummels D, Beekman E, Theunissen K, et al. Nine commercially available activity trackers in people with a chronic disease for activities of daily living: a cross-sectional validity study. JMIR Mhealth Uhealth. 2018;6:e70.

12. Kooiman TJ, Dontje ML, Sprenger SR, et al. Reliability and validity of ten consumer activity trackers. BMC Sports Sci Med Rehabil. 2015;7:24.

13. Ferguson $\mathrm{T}$, Rowlands $\mathrm{AV}$, Olds $\mathrm{T}$, et al. The validity of consumer-level, activity monitors in healthy adults worn in free-living conditions: a cross-sectional study. Int J Behav Nutr Phys Act 2015;12:42.

14. Van Remoortel H, Giavedoni S, Raste $\mathrm{Y}$, et al. Validity of activity monitors in health and chronic disease: a systematic review. Int J Behav Nutr Phys Act. 2012;9:84.

15. Epstein DA, Kang JH, Pina LR, et al. Reconsidering the device in the drawer: lapses as a design opportunity in personal informatics. Ubicomp; 2016 Sep 12-16; Heidelberg, Germany.

16. Lazar A, Koehler C, Tanenbaum J, et al. Why we use and abandon smart devices. Ubicomp; 2015 Sep 711; Osaka, Japan.

17. Harrison D, Marshall P, Bianchi-Berthouze N, et al. Activity tracking: barriers, workarounds and customisation. Ubicomp; 2015 Sep 7-11; Osaka, Japan.

18. Shih PC, Han K, Poole ES, et al. Use and adoption challenges of wearable activity trackers. iConference; 2015 Mar 24-27; Newport Beach, California, USA.

19. Arma_gan K, C,i_gdem E. Design for physical activity: design aspects of wearable activity trackers. International Conference of Engineering Design; 2015 Jun 27-30; Milano, Italy.

20. Maher C, Ryan J, Ambrosi C, et al. Users' experiences of wearable activity trackers: a cross-sectional study. BMC Public Health. 2017;15:17880.

21. Hermsen S, Moons J, Kerkhof P, et al. Determinants for sustained use of an activity tracker: observational study. JMIR Mhealth Uhealth. 2017;5:e164.

22. Karapanos E, Gouveia R, Hassenzahl M, et al. Wellbeing in the making: peoples' experiences with wearable activity trackers. Psychol Well Being. 2016;6:4. 
23. Fausset CB, Mitzner TL, Price CE, et al. Older adults' use of and attitudes toward activity monitoring technologies. Proceedings of the human factors and ergonomics society; 2013 Sep 30-Oct 4; San Diego, CA, USA.

24. O'Brien T, Troutman-Jordan M, Hathaway D, et al. Acceptability of wristband activity trackers among community dwelling older adults. Geriatr Nurs. 2015;36:21-25.

25. Rasche $\mathrm{P}$, Schäfer $\mathrm{K}$, Theis $\mathrm{S}$, et al. Age-related usability investigation of an activity tracker. IJHFE. 2016;4:187.

26. Preusse KC, Mitzner TL, Fausset CB, et al. Older adults' acceptance of activity trackers. J Appl Gerontol. 2017;36: 127-155.

27. Schlomann A. A case study on older adults' long-term use of an activity tracker. Gerontechnology. 2017;16:115-124.

28. Puri A, Kim B, Nguyen $\mathrm{O}$, et al. User acceptance of wristworn activity trackers among communitydwelling older adults: mixed method study. JMIR Mhealth Uhealth. 2017;5:e173.

29. Mercer K, Giangregorio L, Schneider E, et al. Acceptance of commercially available wearable activity trackers among adults aged over 50 and with chronic illness: a mixedmethods evaluation. JMIR Mhealth Uhealth. 2016;4:e7.

30. Rosenberg D, Kadokura EA, Bouldin ED, et al. Acceptability of Fitbit for physical activity tracking within clinical care among men with prostate cancer. AMIA Annu Symp Proc; 2016 Nov 4-8; Washington, USA.

31. Lincoln YS, Guba EG. Naturalistic inquiry. USA: Sage publications; 1985.

32. Palinkas LA, Horwitz SM, Green CA, et al. Purposeful sampling for qualitative data collection and analysis in mixed method implementation research. Adm Policy Ment Health. 2015;42:533-544.

33.. Beekman E, Braun SM, Ummels $D$, et al. Validity, reliability and feasibility of commercially available activity trackers in physical therapy for people with a chronic disease: a study protocol of a mixed methods research. Pilot Feasibility Stud. 2017;3.

34. Agusti A, Decramer M, Celli BR, et al. Pocket guide to COPD diagnosis, management and prevention. Global Initiative for Chronic Obstructive Lung Disease. 2017.

35. Marshall AL, Smith BJ, Bauman AE, et al. Reliability and validity of a brief physical activity assessment for use by family doctors. Br J Sports Med. 2005;39:294-297.

36. Linn BS, Linn MW, Gurel L. Cumulative illness rating scale. J Am Geriatr Soc. 1968;16:622-626.

37. de Groot V, Beckerman H, Lankhorst GJ, et al. How to measure comorbidity. A critical review of available methods. J Clin Epidemiol. 2003;56:221-229.

38. Kallio $\mathrm{H}$, Pietila AM, Johnson $\mathrm{M}$, et al. Systematic methodological review: developing a framework for a qualitative semi-structured interview guide. J Adv Nurs. 2016;72:2954-2965.

39. Kreuger RA, Casey MA. Focus Groups a practial guide for applied research. Vol. 5. USA: SAGE Publications; 2015.

40. Gale NK, Heath G, Cameron E, et al. Using the framework method for the analysis of qualitative data in multidisciplinary health research. BMC Med Res Methodol. 2013;13:117.

41. Elo S, Kyngas H. The qualitative content analysis process. J Adv Nurs. 2008;62:107-115.

42. van Bommel M, Van Gisbergen T. De eerste stap zetten. De hanteerbaarheid en toepasbaarheid van activiteitenmeters [bachelor thesis]. Heerlen (Netherlands): Zuyd University of Applied Science; 2015.

43. Verwey R. Get moving! Self-management support using mobile technology. A counselling protocol extended with a web-based coaching system to promote physical activity in patients with COPD or type 2 diabetes in primary care: the It's LiFe! study [dissertation]. Maastricht: Maastricht University; 2015.

44. Mancuso PJ, Thompson M, Tietze M, et al. Can patient use of daily activity monitors change nurse practitioner practice? J Nurse Pract. 2014;10:787-793.

45. Andrieu N, Mannens L, Nowak K. Toepasbaarheid en hanteerbaarheid van activiteitenmeters binnen de ergotherapie bij COPD-patiënten [bachelor thesis]. Heerlen (Netherlands): Zuyd University of Applied Science; 2015.

46. Lyons EJ, Lewis ZH, Mayrsohn BG, et al. Behavior change techniques implemented in electronic lifestyle activity monitors: a systematic content analysis. J Med Internet Res. 2014;16:e192.

47. Stevens A, Beurskens A, Koke A, et al. The use of patientspecific measurement instruments in the process of goalsetting: a systematic review of available instruments and their feasibility. Clin Rehabil. 2013;27:1005-1019. 


\section{Supplement file S3.1}

\section{Interview Guide}

\section{Introduction:}

- Introduce yourself (name, function)

- Explain you would like to record this interview with a memo recorder

- Explain that everything the patients says will be used without their name

- Ask if they agree with recording the interview

- Ask if they have any question based on the information you just provided

\section{Question 1:}

Which activity tracker did you use?

\section{Question 2:}

Which information did you receive from your physiotherapist before using the activity tracker?

\section{Question 3:}

Can you briefly explain how you experienced the use of the activity tracker?

\section{Question 4:}

With witch purpose was the activity tracker used in your therapy?

Sub question: Did you discussed the results in with your physiotherapist?

Continue with: How did you discussed these results? OR How would you have liked to discuss these results?

Sub question: How can an activity tracker be used in your therapy to improve the results of the therapy?

\section{Question 5:}

Would you purchase an activity tracker?

In case the participants wants to buy an activity tracker:

- If you would buy an activity tracker, which information you would like to know beforehand?

- How would you want to compare this with other activity trackers? (for example wearing location, price and feasibility) 
- What would be the main reason whether to buy or not buy an activity tracker?

- How would an aid to help you choose an activity tracker look appealing to you?

In case the participants does not want to buy an activity tracker:

- If you could borrow an activity tracker from the physiotherapist practice which information you would like to know beforehand?

- How would you want to compare this with other activity trackers? (for example wearing location, price and feasibility)

- What would be the main reason whether to buy or not buy an activity tracker?

- How would an aid to help you choose an activity tracker look appealing to you?

\section{Question 6:}

Most data of activity trackers is stored online. Do you have any objections if other people could see your data?

Sub question: If yes, why? If no, why not?

Sub question Who would be allowed to see your data and who isn't?

\section{Question 7:}

Are there any important issues to you which we haven't discussed during this interview? 


\section{Supplement file S3.1}

\begin{tabular}{|c|c|c|}
\hline Category & Subcategory & Number of quotes \\
\hline \multirow[t]{6}{*}{ Purchase } & Costs of the activity tracker ${ }^{1}$ & 14 \\
\hline & Costs of a subscription ${ }^{2}$ & 2 \\
\hline & Compensation of healthcare insurance ${ }^{2}$ & 3 \\
\hline & Possession of a smartphone ${ }^{1}$ & 7 \\
\hline & Possession of a computer ${ }^{2,3}$ & 14 \\
\hline & Available and clear information about the feasibility of the trackers ${ }^{1,2}$ & 5 \\
\hline \multirow[t]{3}{*}{ Instruction } & Required instruction from physiotherapist ${ }^{1,2}$ & 75 \\
\hline & Support (helpdesk, blogs, YouTube) ${ }^{2,4,5}$ & 0 \\
\hline & Required technical skills ${ }^{2,6-8}$ & 52 \\
\hline \multirow{9}{*}{$\begin{array}{l}\text { Characteristics of } \\
\text { the activity } \\
\text { tracker }\end{array}$} & Installing and receiving data from the activity tracker ${ }^{1,5,7}$ & 59 \\
\hline & Measured variables by the activity tracker ${ }^{2,3,6,7,9,10}$ & 108 \\
\hline & Interface ${ }^{5}$ & 1 \\
\hline & Accessibility ${ }^{2,5}$ & 104 \\
\hline & Wearing comfort ${ }^{1,3,5,7,8,11-13}$ & 155 \\
\hline & Setting goals $3,8,14,15$ & 11 \\
\hline & Complexity $2,6,8$ & 131 \\
\hline & Feedback $k^{1,3,6-11,13}$ & 135 \\
\hline & Robustness ${ }^{2,5,13}$ & 29 \\
\hline Correct & Validity ${ }^{5-7,9,11-13}$ & 11 \\
\hline \multirow[t]{2}{*}{ functioning } & Reliability ${ }^{5-7,9,11-13}$ & 30 \\
\hline & Technical errors ${ }^{2,4}$ & 23 \\
\hline \multirow[t]{2}{*}{ Sharing data } & Interoperational $\left.\right|^{2,3,5}$ & 0 \\
\hline & Possibility to share data $a^{2,4,7,12,14}$ & 8 \\
\hline \multirow[t]{4}{*}{ Privacy } & Safely sharing data ${ }^{5,7,11,13}$ & 2 \\
\hline & Warrant of privacy ${ }^{2}$ & 22 \\
\hline & Insight into physical activity level by physiotherapist ${ }^{11}$ & 12 \\
\hline & Authorisation, authentication, license ${ }^{2}$ & 0 \\
\hline Use of the activity & Implementation in physiotherapy ${ }^{1,2,4}$ & 49 \\
\hline \multirow{5}{*}{ tracker } & Interface ${ }^{5}$ & 0 \\
\hline & Compliance $^{2,4}$ & 89 \\
\hline & Setting goals $s^{4,6,15}$ & 129 \\
\hline & Choice of activity tracker & 30 \\
\hline & Discussing results with physiotherapist & 38 \\
\hline Interest in & Added value of feedback ${ }^{1,3,6,8-11,16}$ & 184 \\
\hline feedback & Faith in measurements and measurement procedures $2,5,7,9,13$ & 39 \\
\hline
\end{tabular}

* Subtopics in bold are added after the evaluation of the framework

\section{References}

1. van Bommel M, Van Gisbergen T. De eerste stap zetten. De hanteerbaarheid en toepasbaarheid van activiteitenmeters. [bachelor thesis] Heerlen (Netherlands): Zuyd University of Applied Science; 2015.

2. Expertmeeting

3. Armağan K, Çiğdem E. Design for physical activity: design aspects of wearable activity trackers. International Conference of Engineering Design; 2015 Jun 27-30: Milano, Italy.

4. Verwey R. Get moving! Self-management support using mobile technology. A counselling protocol extended with a web-based coaching system to promote physical activity in patients with COPD or type 2 diabetes in primary care: the It's LiFe! study. [dissertation] Maastricht: Maastricht University 2015. 
5. Mancuso PJ, Thompson M, Tietze M, et al. Can Patient Use of Daily Activity Monitors Change Nurse Practitioner Practice? The Journal for Nurse Practitioners. 2014;10(10):787- 793.

6. $\quad$ Lazar A, Koehler C, Tanenbaum J, et al. Why we use and abandon smart devices. Ubicomp; 2015 Sept 711: Osaka, Japan.

7. Rosenberg D, Kadokura EA, Bouldin ED, et al. Acceptability of Fitbit for physical activity tracking within clinical care among men with prostate cancer. AMIA Annu Symp Proc. 2016 Nov 4-8: Washington, USA.

8. Mercer K, Giangregorio L, Schneider E, et al. Acceptance of Commercially Available Wearable Activity Trackers Among Adults Aged Over 50 and With Chronic Illness: A Mixed-Methods Evaluation. JMIR Mhealth Uhealth. 2016:4(1):e7.

9. Fausset CB, Mitzner TL, Price CE, et al. Older Adults' Use of and Attitudes toward Activity Monitoring Technologies. Proceedings of the human factors and ergonomicssociety; 2013 Sep 30- Oct 4: San Diego, USA.

10. O'Brien T, Troutman-Jordan M, Hathaway D, et al. Acceptability of wristband activity trackers among community dwelling older adults. Geriatr Nurs. 2015:36(2):21-5.

11. Andrieu N, Mannens L, Nowak K. Toepasbaarheid en hanteerbaarheid van activiteitenmeters binnen de ergotherapie bij COPD-patiënten. [bachelor thesis] Heerlen: Zuyd University of Applied Science; 2015.

12. Harrison D, Marshall P, Bianchi-Berthouze N, et al. Activity tracking; Barriers, Workarounds and Customisation. Ubicomp; 2015 Sept 7-11: Osaka, Japan

13. Shih PC, Han K, Poole ES, et al. Use and Adoption Challenges of Wearable Activity Trackers. iConference;2015 March 24-27: Newport Beach, California, USA.

14. Lyons EJ, Lewis ZH, Mayrsohn BG, et al. Behavior change techniques implemented in electronic lifestyle activity monitors: a systematic content analysis. J Med Internet Res. 2014;16(8):e192.

15. Stevens A, Beurskens A, Koke A, et al. The use of patient-specific measurement instruments in the process of goal-setting: a systematic review of available instruments and their feasibility. Clin Rehabil. 2013;27(11):1005-1019.

16. Karapanos E, Gouveia R, Hassenzahl M, et al. Wellbeing in the Making: Peoples' Experiences with Wearable Activity Trackers. Psychol Well Being. 2016;6:4. 
4 


\section{Chapter 4}

Measure It Super Simple (MISS) activity tracker:

(re)design of a user-friendly interface and

evaluation of experiences in daily life

Darcy Ummels, Susy Braun, An Stevens, Emmylou Beekman \& Anna Beurskens

Disability Rehabilitation Assistive Technology. 2020 Sep 24:1-11 


\section{Abstract}

\section{Purpose}

The purposes of this study were, first, to (re)design the user-interface of the activity tracker known as the MOX with the help of input from elderly individuals living independently and, second, to assess the use of and experiences with the adapted Measure It Super Simple (MISS) activity tracker in daily life.

\section{Methods}

The double diamond method, which was used to (re)design the user-interface, consists of four phases: discover, define, develop, and deliver. As a departure point, this study used a list of general design requirements that facilitate the development of technology for the elderly. Usage and experiences were assessed through interviews after elderly individuals had used the activity tracker for 2 weeks.

\section{Results}

In co-creation with thirty-five elderly individuals (65 to 89-years-old) the design, feedback system, and application were further developed into a user-friendly interface: the Measure It Super Simple (MISS) activity. Twenty-eight elderly individuals (65 to 78 years-old) reported that they found the MISS activity easy to use, needed limited help when setting the tracker up, and required limited assistance when using it during their daily lives.

\section{Conclusions}

This study offers a generic structured methodology and a list of design requirements to adapt the interface of an existing activity tracker consistent with the skills and needs of the elderly. The MISS activity seemed to be successfully (re)designed, like the elderly who participated in this pilot study reported that anyone should be able to use it. 


\section{Introduction}

Smart health has become increasingly popular in recent years as a method of monitoring physical activity, as well as health- and lifestyle-related variables. ${ }^{1}$ The term smart health refers to mobile applications (apps), wearables, and smartwatches. ${ }^{1}$ Although activity trackers are mainly used by a young and physically fit population, the elderly could also benefit from the use of an activity tracker. In the Netherlands, 53\% of elderly individuals $(65+)$ do not meet the Dutch physical activity guideline ${ }^{2,3}$ even though there is ample evidence that sufficient physical activity can prevent several chronic diseases, reduce the risk of premature death, and decrease the risk of fractures and falls in the elderly., ${ }^{4,5}$ Barriers that inhibit elderly individuals' physical activity noted in the literature include a lack of motivation and self-discipline, the assumption that they are already sufficiently active, limited knowledge about the positive effects of physical activity, and a lack of habits. ${ }^{6,7}$ Studies show that incorporating the use of an activity tracker into one's healthcare can motivate elderly individuals to become more physically active. The primary explanations for this phenomenon are that trackers provide insight into an elderly individual's amount of physical activity and increase their awareness of their physical activity. ${ }^{8-13}$

The user-friendliness of an activity tracker is an important feature to start and continue using an activity tracker. Several studies concluded that the elderly do not achieve a meaningful user-experience with existing activity trackers because there is a disparity between tracker features and the skills and needs of the elderly. ${ }^{10-16}$ Frequently mentioned problems include an imbalance between technical skills and the perceived complexity of the activity tracker, the lack of a clear manual, complex interpretation of feedback, excessively high standards for daily activity goals (e.g., 10,000 steps), and reduced wearing comfort. ${ }^{10-16}$

Consequently, there is a need for an activity tracker that meets the needs and skills of the elderly. In this study, we wanted to examine whether the user-interface of an existing activity tracker could be adapted in order to facilitate a (more) meaningful experience for the elderly. An example of an activity tracker whose user-interface can be adapted is the MOX Activity Monitor (MOX ${ }^{17}$, Box 4.1).

Since the algorithm settings of the MOX (including the optimized parameter settings of MOX $_{\text {Missactivity) }}$ have been validated for an elderly population ${ }^{18}$, we wanted to examine whether the user-interface of the MOX could be adapted for this target group as well. In this article, the user-interface refers to (1) the design of the tracker's hardware, (2) the feedback system and display of the activity tracker, and (3) the feedback provided by a mobile application. 
Box 4.1 About "MOX Activity Monitor".

- The MOX Activity Monitor contains a tri-axial accelerometer.

- The algorithm of the MOX was recently optimized and the algorithm settings were validated in an elderly

- population.

- $\quad$ The system can measure and transfer physical activity parameters continuously every second for 14 days.

- Dust \& Waterproof IPX8

- CE-certified

- Wireless communication Bluetooth LE

- $\quad$ Battery lifetime 14 days

0

Based on the recent research of Ummels et al. ${ }^{10}$ regarding the experiences of the elderly with eight different activity trackers, 53 important characteristics to create a meaningful user-interface were determined (Appendix 4.1). For instance, three important characteristics from this list include the wearing location, outcome parameters, and feedback options. Thus, findings from Ummels et al. that have been broadly supported by the literature ${ }^{11-13,15,19-32}$ were used as a departure point for the design of the userinterface.

In order to facilitate meaningful user experience and to assess the usage and experiences of the elderly with the activity tracker, this study was conducted with the following objectives:

1. to (re)design a user-friendly interface of the MOX, meeting the requirements of the elderly by using the double diamond method.

2. to assess the elderly's use and experiences of the adapted activity tracker in daily life.

\section{Methods}

This study consists of two methods that aim to answer the two aforementioned research questions (Figure 4.1) and were approved by a local ethics board (METC-Z Medical Ethical Committee, METCZ20180012).

Regarding the (re)design of the user-interface, the double diamond method ${ }^{33}$ was used. The double diamond method consists of four phases: the discovery, definition, development, and delivery phase. The discovery phase was completed in a preliminary 
phase (Appendix 4.1) and served as a departure point for the remaining three phases, which are outlined below.

To assess the use of and experiences with the adapted activity tracker, a sample of elderly individuals using the activity tracker for a period of 2 weeks. Afterward, all participants were interviewed.

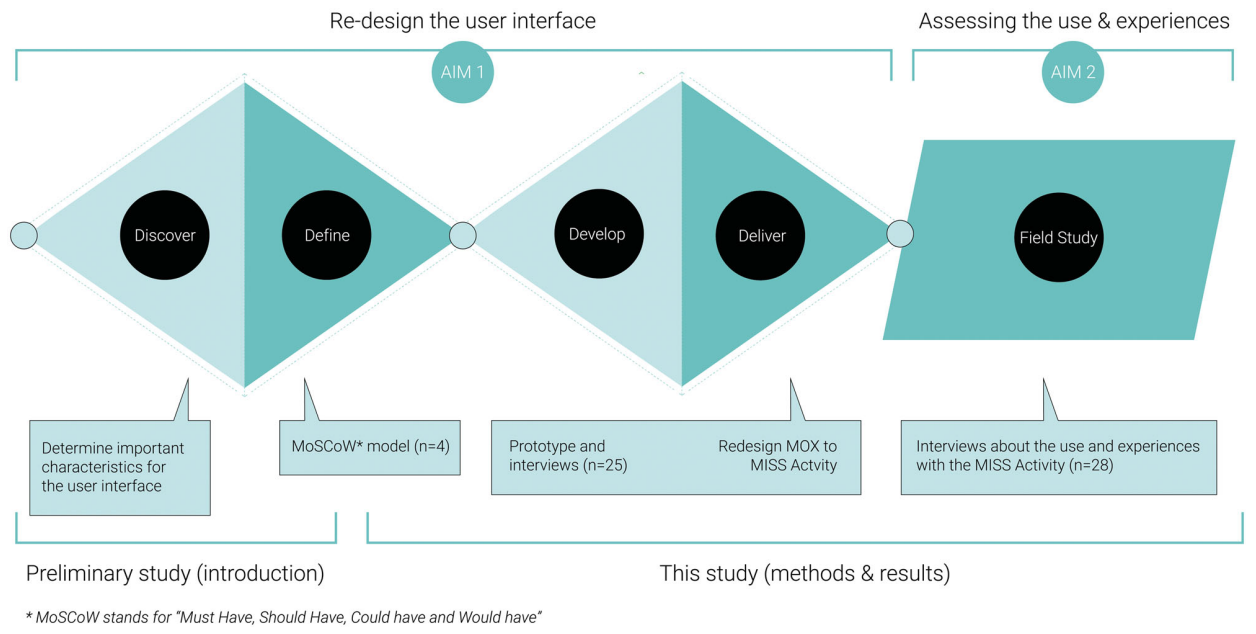

Figure 4.1 Schematic overview of the two designs used in this study.

\section{(Re)design of the user-interface}

\section{Definition phase}

To prioritize the 53 characteristics outlined in the discovery phase, the MoSCoW model was used. ${ }^{34}$ Four experts in wearables (a professor in the field of smart devices, a physiotherapist, an advisor from the knowledge centre for sports and physical activity, and a clinical operator of a human kinetics lab) were asked to prioritize these characteristics, based on technical complexity and market distinctiveness. When at least two experts rated a characteristic as a "should have" the characteristic was labelled critical.

\section{Development and delivery phase}

Based on the definition phase, three key aspects of the activity tracker were included: (1) the design of the activity tracker, (2) the way the activity tracker provides feedback, 
and (3) an app that provides additional feedback. Consequently, each aspect was designed in co-design, described in a user manual, and tested with the elderly.

\section{Participants}

Participants were recruited via several local associations, such as sports clubs and social initiatives for the elderly. Participants were eligible if they were 65-years-old or older and possessed a sufficient understanding of the Dutch language. The following demographic characteristics of the participants were noted: gender, age, knowledge of technology (between 0 and 10), and use of a computer, tablet, smartphone, and activity tracker (Yes/No). All participants provided written informed consent after receiving verbal and written information about the research.

\section{Design of the activity tracker}

Three participants were interviewed about their preferences regarding the design of the activity tracker. They were provided with six distinct activity tracker shapes and clips. They were then asked to arrange their preferences for these shapes and clips. Participants were asked to explain why they chose this sequence. The interviews were audio-taped and summarised, and pictures were taken of the final sequences. Finally, based on the interviews, a clip and shape were designed.

\section{Feedback system on the activity tracker}

Twelve participants (two focus groups) were interviewed about feedback concepts for battery life, progress to goal, and synchronization status (Figure 4.2). Furthermore, they were asked about their opinion on how they would like to activate the feedback on the activity tracker (e.g., tapping or shaking). These interviews were audio-taped and summarised. Based on the results, the feedback system on the activity tracker was adapted and implemented.

\section{Additional feedback by an application}

Twenty participants were asked to perform several tasks on a designed mock-up app. The performance was documented by a researcher (DU). Afterward, the participants were asked about the user-experience. The first 10 participants were also provided with three alternative designs (Figure 4.3), from which they could select their preference. After 10 interviews, the app was adjusted based on the feedback of these first ten participants. Based on the last 10 interviews, the app was adapted to its final version. 
Concepts battery life
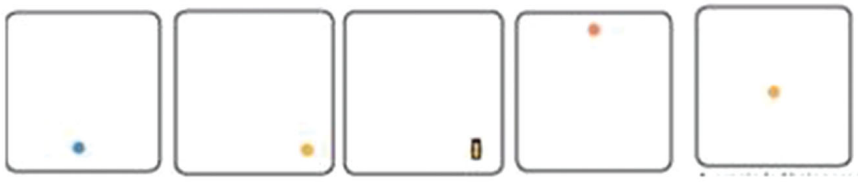

Concepts progress to goal
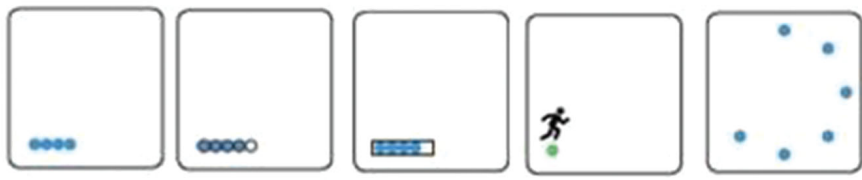

Concepts

synchronisation status
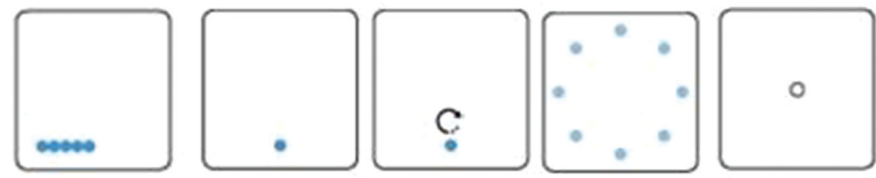

Figure 4.2 Feedback concepts for battery life, progress to goal, and synchronization status.
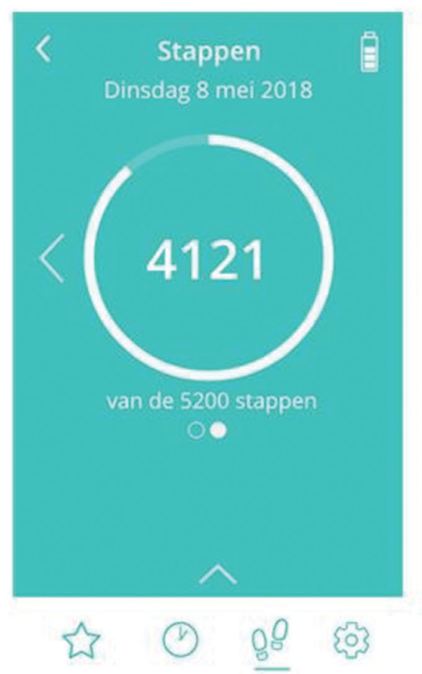

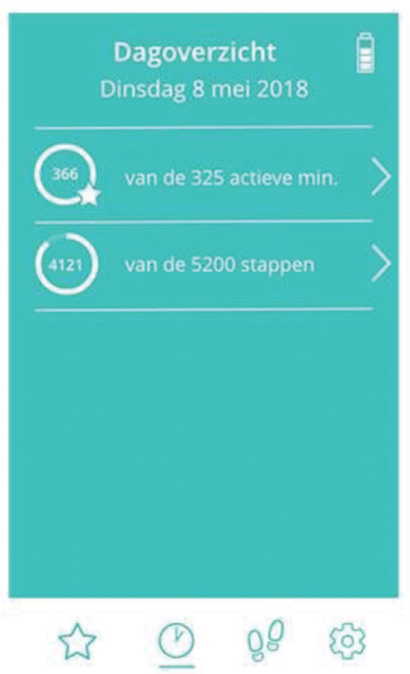

인

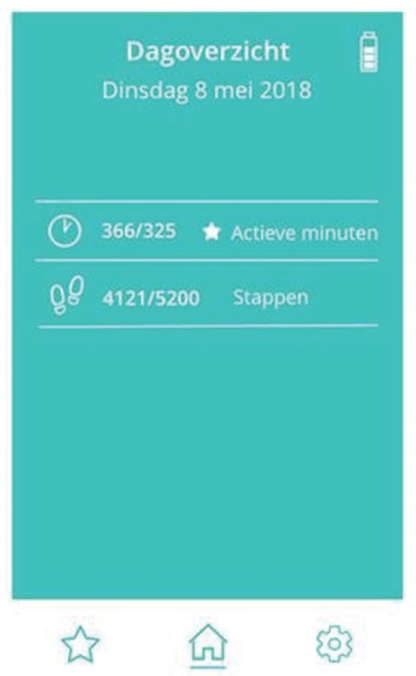

Figure 4.3 Alternative designs for the application.

Evaluating use and experiences in daily life

The adapted user-interface was integrated into the MOX. Together with the developed algorithm ${ }^{18}$, the activity tracker is called the Measure It Super Simple (MISS) activity tracker. $^{35}$ To evaluate if the Measure It Super Simple (MISS) activity was indeed 
successfully (re)designed, thirty participants used the MISS activity for 2 weeks. During this time, two personal visits and two check-up calls were organized (Figure 4.4).

To evaluate the success of the (re)designed MISS activity, thirty participants were asked to use the MISS activity for 2 weeks. During this period, two personal visits and two check-up calls were organized (Figure 4.4).

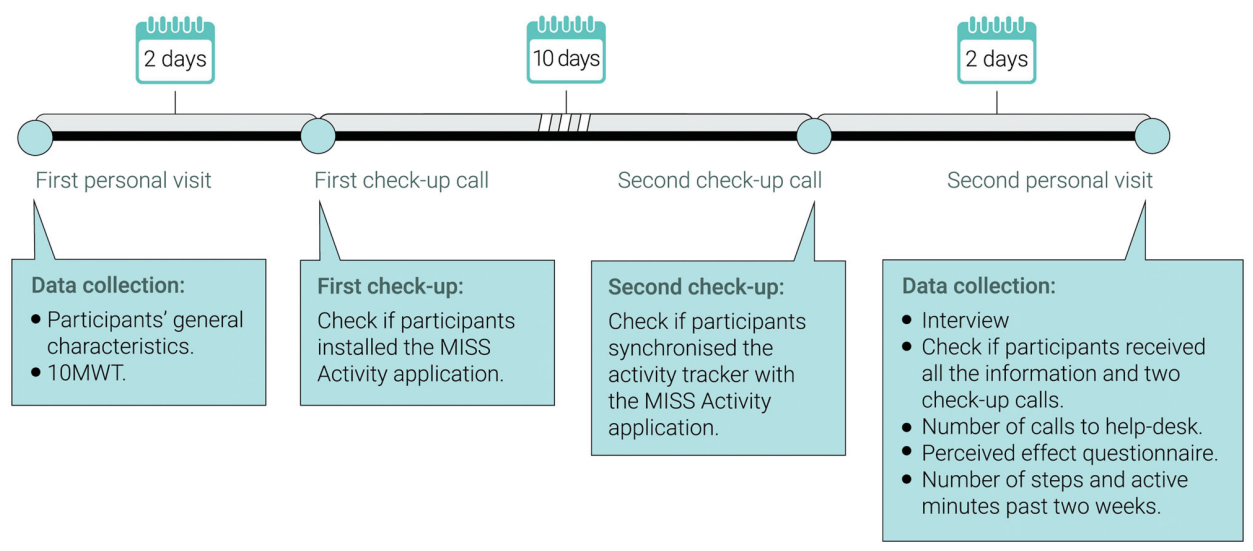

Figure 4.4 Overview of data collection during the 2 weeks test period. Abbreviation - 10MWT: 10-meter walking test.

\section{Participants}

Participants were recruited through convenient sampling via several local associations, such as sports clubs or social initiatives for the elderly. Participants were eligible if they were 65 years or older, had a sufficient understanding of the Dutch language, and possessed a smartphone. Participants were excluded if they had an asymmetrical gait that was observed during the $10-\mathrm{m}$ walking test ${ }^{36}$ or used a walking aid.

\section{First personal visit}

The first personal visit was located at a convenient location for the participant (e.g., their home). General characteristics were collected: gender, age, the highest level of education, living situation, family composition, use of technology, and their hobbies. Participants received a MISS activity, which they were asked to wear for 2 weeks, and a user manual. No further instructions about the use of the MISS activity were given. Participants were invited to call the helpdesk if they perceived any problems. 


\section{Check-up calls}

Two days after the first appointment, a researcher called the participant to check if they had installed the app. The researcher pointed out again, that participants could obtain support through the helpdesk if they encountered problems. After ten days the researcher called the second time to ask if participants had synchronized the activity tracker with the application in the last 2 weeks. If not, they were asked to do so.

\section{Second personal visit}

At the end of the 2 weeks testing period, a second personal visit was scheduled during which the researcher asked whether (1) the participant had received the number of the helpdesk and the user manual, (2) whether the participant had had two check-up calls and (3) how many times the participant had called the helpdesk. The step count and active minutes per day according to the MISS activity app were recorded. Furthermore, one questionnaire was filled out in which the satisfaction with the MISS activity and the effect of their physical activity was queried (Appendix 4.2).

Semi-structured interviews with all participants about their experiences with the MISS activity were performed. The interview guide was based on a framework of a previous study $^{10}$, with the following main categories: purchase, instruction, characteristics, correct functioning, sharing data, privacy, use of the activity tracker, and interest in feedback. The interview lasted between 15-30 min and was recorded on audio.

\section{Data analysis}

Descriptive statistics of the participants' characteristics and questionnaires were presented as absolute numbers with medians (range). The interviews were transcribed verbatim. To analyze the interviews deductive and inductive content analysis ${ }^{37}$ was used, using NVivo (version 10). The framework ${ }^{10}$ of the interview guide was used for the deductive content analysis. Inductive coding was used when a text passage, that was relevant for the research question, did not fit the framework. Therefore, an "other" code was used to include these text passages. The first interview and every fifth interview was coded by two reviewers and an alignment session was held to fine-tune the coding. Differences in interpretation were solved by dialogue to reach consensus. 


\section{Results}

\section{(Re)design of the user-interface}

\section{Definition phase}

From the 53 characteristics, 31 were prioritized as critical based on the MoSCoW model. Five of these characteristics were related to the algorithm of the activity tracker. The remaining 26 characteristics were related to the user-interface and used in de development and delivery phases (Appendix 4.1).

\section{Development and delivery phase}

\section{Participants}

The participants' demographic characteristics are presented in Table 4.1.

Table 4.1 Demographics of the included participants of the develop and deliver phase.

\begin{tabular}{lccc}
\hline Characteristics & $\begin{array}{c}\text { Participants shape } \\
\text { and wear location } \\
(n=3)\end{array}$ & $\begin{array}{c}\text { Participants } \\
\text { feedback } \\
(n=12)\end{array}$ & $\begin{array}{c}\text { Participants app } \\
(n=20)\end{array}$ \\
\hline Gender, male, n (\%) & $2(66)$ & $6(50)$ & $10(50)$ \\
Age (year), median (range) & $66(66-74)$ & $71(66-77)$ & $72.5(65-89)$ \\
Knowledge of technology ${ }^{a}$, median, (range) & $7(5.5-8)$ & $7(0-7)$ & $6(0-8)$ \\
Use of technology, $n(\%)$ & & & $15(75)$ \\
Smartphone & $2(66)$ & $4(33)$ & $15(75)$ \\
Computer & $3(100)$ & $11(92)$ & $8(40)$ \\
Tablet & $2(66)$ & $6(50)$ & $5(25)$ \\
Activity tracker & $1(33)$ & $3(25)$ & \\
\hline
\end{tabular}

a Participant could score their knowledge of technology between zero and ten. Zero representing no knowledge and 10 a lot of knowledge.

The main features of the development and delivery phase are presented in Table 4.2. These findings combined resulted in the MISS activity tracker (Box 4.2).

\section{Evaluating usage and experiences in daily life}

\section{Participants}

The demographic characteristics of the participants are presented in Table 4.3. Two participants dropped out ( $n=2$ : participant's smartphone was too outdated to run the MISS activity app and one due to illness). 
Table 4.2 Main finding of the development and delivery phase.

\begin{tabular}{|c|c|c|}
\hline Characteristic & Main Findings & Remarks \\
\hline Design of the activity tracker & $\begin{array}{l}\text { - Activity tracker should be round } \\
\text { - Activity tracker should be worn on } \\
\text { one's pants pocket with a clip } \\
\text { - Clip should be long enough } \\
\text { - Clip should be sturdy } \\
\text { - Clip should be easy to put on }\end{array}$ & $\begin{array}{l}\text { A round shape was technically not } \\
\text { possible, therefore a square shape } \\
\text { was chosen }\end{array}$ \\
\hline $\begin{array}{l}\text { Feedback system on the activity } \\
\text { tracker }\end{array}$ & $\begin{array}{l}\text { - Colour of the hardware should be } \\
\text { - } \text { Beutral } \\
\text { - } \text { Colour of lights on activity tracker is } \\
\text { not important } \\
\text { - Feedback as simple as possible } \\
\text { - No feedback about synchronization } \\
\text { - Feedback activation of activity } \\
\text { tracker by tapping on tracker }\end{array}$ & $\begin{array}{l}\text { It was technically not possible to } \\
\text { activate the activity tracker by } \\
\text { tapping, therefore the activity } \\
\text { tracker will be activated by shaking }\end{array}$ \\
\hline Feedback by an app & $\begin{array}{l}\text { - Good user-flow is needed } \\
\text { - Original design was preferred } \\
\text { - App should work both clicking as } \\
\text { well as swiping } \\
\text { - Additional information screen is } \\
\text { needed }\end{array}$ & \\
\hline
\end{tabular}

Box 4.2 About the MISS activity tracker.

- The square design was worn on the pocket and secured with a clip

- Algorithm outcome parameters: step count and active minutes

- Users can choose whether the standing activity is included as active minutes

- Feedback on battery level and progress to goal with LED lights on the activity tracker itself

- Feedback is activated by shaking the activity tracker

- The app shows total step count and total active minutes

- The app shows a graph with the distribution of step count and active minutes during the day

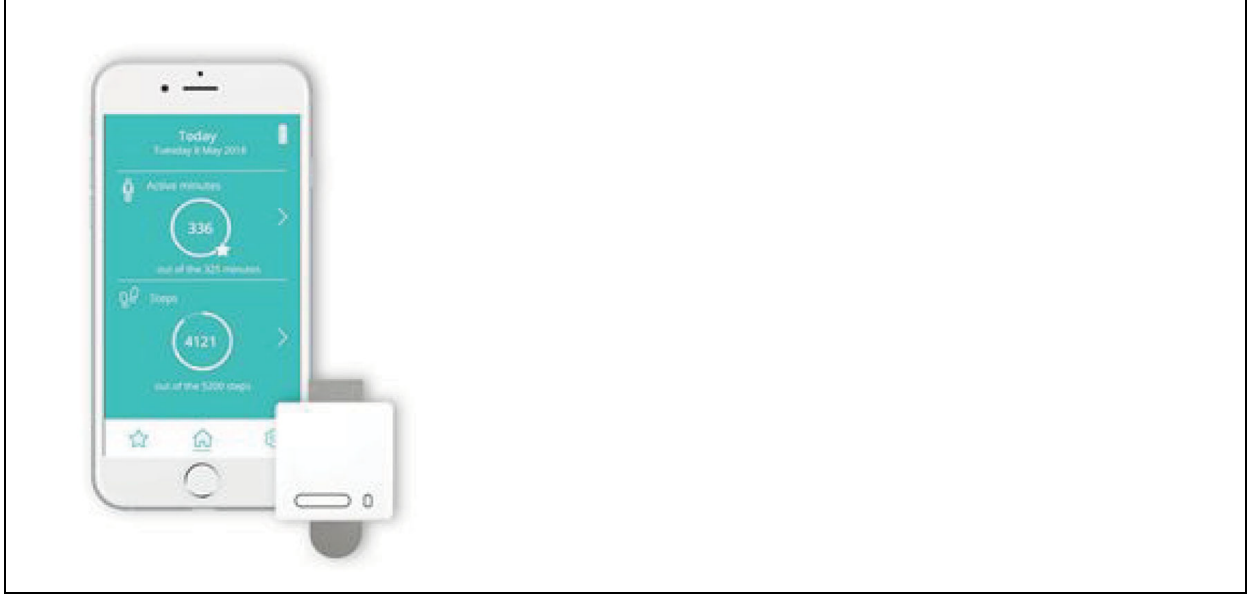


Table 4.3 Demographics of the included participants during the 2-week testing period.

\begin{tabular}{|c|c|}
\hline Characteristics & Participants $(n=28)$ \\
\hline Gender, male, n (\%) & $11(39)$ \\
\hline Age (year), median (range) & $69(65-78)$ \\
\hline \multicolumn{2}{|l|}{ Education, $\mathrm{n}(\%)$} \\
\hline Secondary Education & $12(40)$ \\
\hline College & $14(50)$ \\
\hline University 3 (10) & $14(50)$ \\
\hline \multicolumn{2}{|l|}{ Living situation, } \\
\hline Living alone & $2(6)$ \\
\hline Living with a partner & $23(81)$ \\
\hline Living with partner and child(ren) & $4(13)$ \\
\hline \multicolumn{2}{|l|}{ Housing situation, $\mathrm{n}(\%)$} \\
\hline Single-family dwelling & $28(100)$ \\
\hline \multicolumn{2}{|l|}{ Hobbies $^{a}, \mathrm{n}(\%)$} \\
\hline Active & $48(47)$ \\
\hline Passive & $54(53)$ \\
\hline \multicolumn{2}{|l|}{ Use of technology, n (\%) } \\
\hline Computer & $27(96)$ \\
\hline Tablet & $24(86)$ \\
\hline Activity tracker & $11(39)$ \\
\hline \multicolumn{2}{|l|}{ Operating systems smartphone, $\mathrm{n}(\%)$} \\
\hline Apple & $12(43)$ \\
\hline Android & $17(57)$ \\
\hline
\end{tabular}

\section{Interviews}

All participants received the manual, as well as the number of the helpdesk, and were called twice. Of the 28 participants, 27 (96\%) used the manual, and seven (24\%) used it more than once. Five (16\%) participants called the helpdesk. One participant asked a question about Bluetooth, one asked about the graphs, and one asked if he could swim with the MISS activity. Two participants had a question about installing the app. One participant did not use the MISS activity for four days since his step count and active minutes were zero. Fourteen participants chose to include standing as active minutes.

Eleven (42\%) participants were absolutely satisfied with the MISS activity, nine (35\%) were very satisfied, four (16\%) were somewhat satisfied and two (7\%) participants were neutral ( $n=26,2$ missing participants). Eleven (42\%) participants gained much more insight into their physical activity level, six (16\%) gained a little more insight, and eleven (42\%) did not gain any insight. One (3\%) participant increased his physical activity level much, three (11\%) participants increased their physical activity level a little, and 24 (86\%) did not perceive any increase. One participant (3\%) noticed that he divided his activities much more over the day, six (22\%) participant divided their activities a little more over the day and 21 (75\%) participants did not perceive any change. The results of the interviews are shown in Table 4.4. 


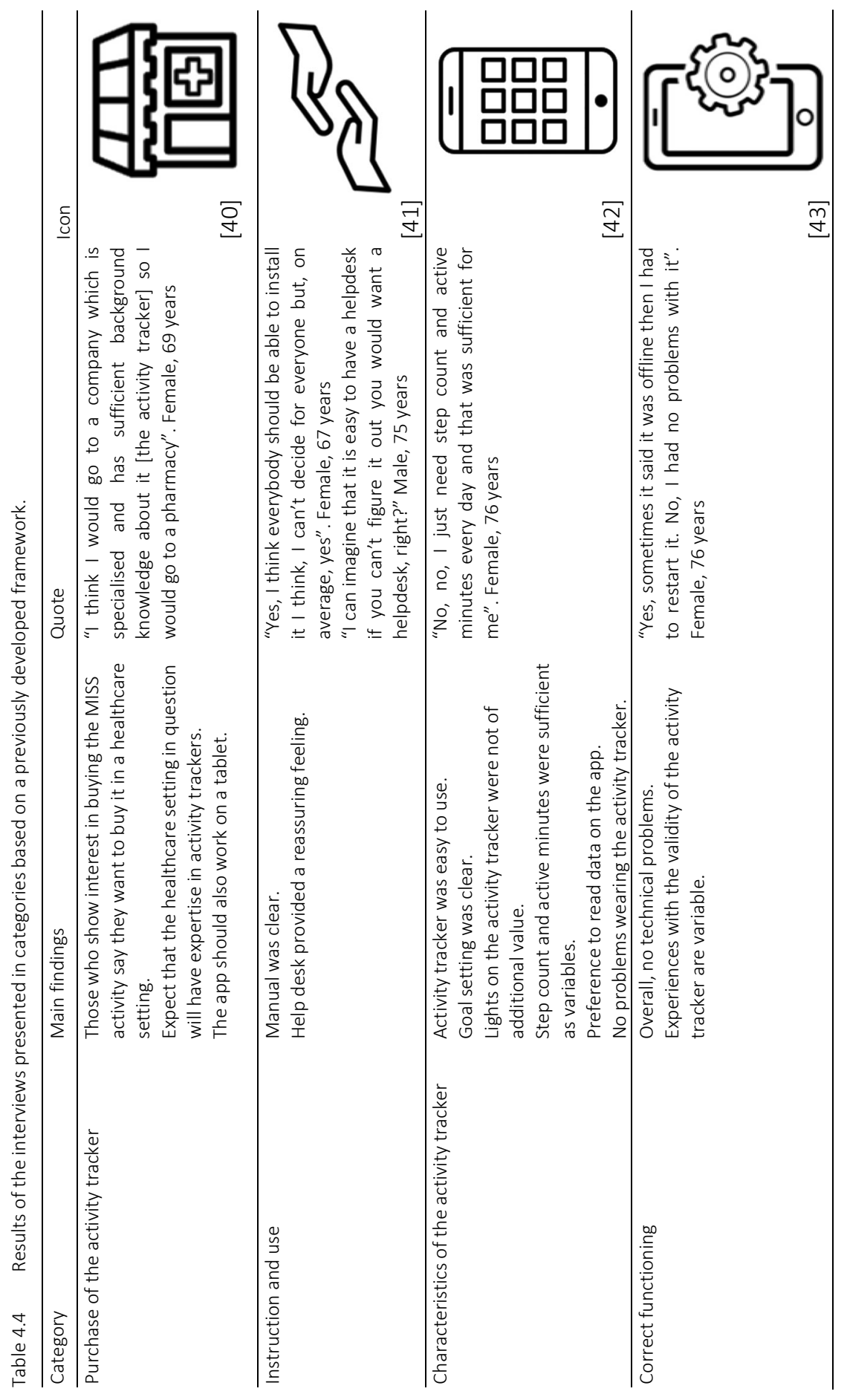

(Re)design of a user-friendly interface andevaluation of experiences in daily life | 81 


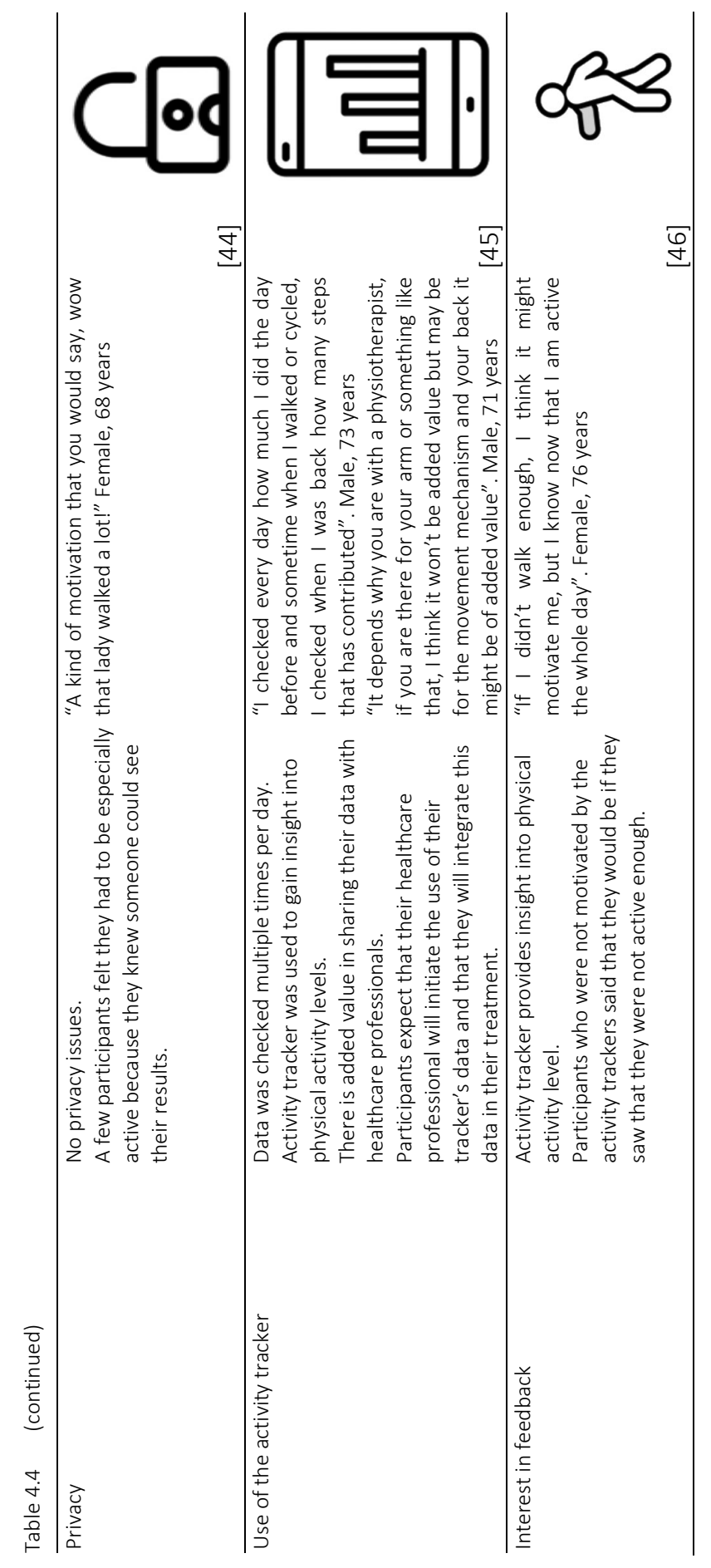




\section{Discussion}

The aim of this study was twofold. The first aim was to (re)design a user-friendly interface of an existing activity tracker so that it would meet the requirements of elderly individuals. Within the (re)design process of the MOX, three aspects were (re)designed: (1) the shape and clip of the activity tracker, (2) the feedback provided on the activity tracker itself, and (3) the additional feedback provided through the app. Furthermore, a list of general design requirements that facilitate the development of technology for the elderly was developed. The iterative character of the user-centred approach enabled access to a deeper level of user understanding and thereby facilitated the development of a user-friendly activity tracker, which in turn increases the likelihood that elderly individuals will have a meaningful experience with the tracker.

The second aim of this study was to assess the use and experiences of the elderly regarding the adapted activity tracker (MISS activity) in their daily life. The results of this study show that participants found the MISS activity to be easy to use, needed limited help when installing the tracker, and thought that anyone should, in principle, be able to use it. The MISS activity was primarily used to gain insight into participants' physical activity levels, and the data it collected was checked multiple times each day through the app. The most important distinction between this tracker and others is that elderly individuals experienced the MISS activity as easy to use; they perceived practically no imbalance between their technical knowledge and the complexity of the MISS activity. In several other studies in which elderly individuals used commercially available activity trackers, participants experienced the trackers as technically complex and sensed that they were ill-suited not fitted for them. ${ }^{10-16}$

\section{Limitations and strengths}

This study contains several strengths and limitations that should be addressed. One strength of this study is its user-centred design. The goal of user-centred design is to create a usable system that contributes to meaningful user experience. To achieve this, (early) involvement of the end-user is indispensable ${ }^{47}$, and offers several benefits, such as a swifter acceptance of the user-interface, the capacity for users to identify problems specific to them, and the capacity for users to help define the scope of a project. ${ }^{47}$ In this study, several methods have been used - interviews, observations, and usability testing ${ }^{48}$ - to achieve a deeper understanding of the specific wishes and requirements of the elderly.

Given that a plethora of commercially available activity trackers already exists, a further strength of this study is the fact that it improved an existing activity tracker for a specific 
target group rather than design a new activity tracker for an overrun market. This study's methodology and list of design requirements can easily be used as a generic structured methodology for improving further eHealth tools for target groups. Furthermore, the synopsis of key characteristics could also be applied to further eHealth and mHealth tools for the elderly.

A limitation of this study is the potential presence of selection bias, as the only elderly individuals who participated already actively used their smartphones. Of all elderly individuals in the Netherlands in 2019 (3.2 million) ${ }^{49}, 60 \%$ use a smartphone. ${ }^{50}$ However, the elderly participating in this pilot study asserted that anyone should, in principle, be capable of using the tracker. The MISS activity is designed for elderly individuals who are interested in using eHealth to track their health, and it is, therefore, most likely the group of elderly who already actively use their smartphone.

\section{Implications for healthcare and research}

Activity trackers can be valuable to healthcare because they objectively measure physical activity throughout daily activities and provide detailed feedback. However, until now, the benefits of activity trackers had not been fully realized. A potential explanation for this could be the limited feasibility of current commercially available activity trackers for both patients and healthcare professionals. ${ }^{10}$ Additional explanations include the overwhelmingly broad spectrum of activity trackers, the lack of consumer and healthcare professional knowledge regarding which activity tracker best suits which purpose and which group, and the lack of professional knowledge regarding how to implement tracker data into healthcare treatment. This study, however, provides an overview of the features that are most relevant for the elderly, and it can be used to facilitate the process of selecting an appropriate tracker.

Further research could examine whether the MISS activity could also be used by other target groups, such as people with limited health literacy, and whether the MISS activity could be used for an extended duration. Ultimately, activity trackers and eHealth could be better implemented in healthcare. ${ }^{51}$ Activity trackers may serve several purposes in healthcare; they can be used to monitor a patient's physical activity level, to set physical activity goals, and to evaluate the physical activity of a patient. Therefore, future studies should explore methods of using activity trackers to support healthcare professionals' clinical reasoning, as well as their communication with their patients. 


\section{Conclusion}

This study offers a structured methodology that facilitates the adaptation of an existing activity tracker. It also describes a list of design requirements based on the skills and needs of the elderly. Both can be used by other researchers and designers as a model for the adaptation and further development of eHealth tools for a target group. The MOX was successfully (re)designed to meet with the skills and needs of the elderly participating in this study. Further studies should explore methods of incorporating activity trackers into the healthcare system.

\section{Acknowledgments}

We would like to thank Jos Aarts, Jolien Dabekaussen, Gaston Jamin, Erik Sauvé, and Frank Helgers for their contributions during this study. 


\section{References}

1. Renders C, Boots T, Visser K, et al. Smart health monitor: onderzoek naar gebruik van apps, wearables en meters. 1st ed. 's-Hertogenbosch (The Netherlands): Multiscope; 2016.

2. National Institute for Public Health and the Environment [Internet]. Bilthoven (The Nethwrlands): National Institute for Public Health and the Environment; 2017 [cited 2018 September 26]. Available from: https://www.volksgezondheidenzorg.info/sites/default/files/hoeveel_mensen_voldoen_aan_gr_ beweegrichtlijnen_2017.pdf

3, National Institute for Public Health and the Environment [Internet]. Bilthoven (The Netherlands): National Institute for Public Health and the Environment; 2018 [cited 2019 June 03]. Available from: https://www.rivm.nl/leefstijlmonitor/bewegen

4. Warburton DER, Bredin SSD. Health benefits of physical activity: a systematic review of current systematic reviews. Curr Opin Cardiol. 2017;32(5):541-556.

5. Vogel T, Brechat PH, Lepretre PM, et al. Health benefits of physical activity in older patients: a review. Int J Clin Pract. 2009;63(2):303-320.

6. Costello E, Kafchinski M, Vrazel J, et al. Motivators, barriers, and beliefs regarding physical activity in an older adult population. J Geriatr Phys Ther. 2011;34(3):138-147.

7. Hoogendoorn MP, de Hollander EL. Belemmeringen en drijfveren voor sport en bewegen bij ondervertegenwoordigde groepen. Bilthoven (The Netherlands): National Institute for Public Health and the Environment; 2016.

8. Maher C, Ryan J, Ambrosi C, et al. Users' experiences of wearable activity trackers: a cross-sectional study. BMC Public Health. 2017;17(1):880.

9. Sullivan AN, Lachman ME. Behavior change with fitness technology in sedentary adults: a review of the evidence for increasing physical activity. Front Public Health. 2016;4:289.

10. Ummels D, Beekman E, Moser A, et al. Patients' experiences with commercially available activity trackers embedded in physiotherapy treatment: a qualitative study. Disabil Rehabil. 2019;1-9.

11. O'Brien T, Troutman-Jordan M, Hathaway D, et al. Acceptability of wristband activity trackers among community dwelling older adults. Geriatr Nurs. 2015;36(2):S21-S25.

12. Preusse KC, Mitzner TL, Fausset CB, et al. Older adults' acceptance of activity trackers. J Appl Gerontol. 2017;36(2):127-155.

13. Mercer K, Giangregorio L, Schneider E, et al. Acceptance of commercially available wearable activity trackers among adults aged over 50 and with chronic illness: a mixedmethods evaluation. JMIR Mhealth Uhealth. 2016;4(1):e7.

14. Fausset CB, Mitzner TL, Price CE, et al. Older adults' use of and attitudes toward activity monitoring technologies. Proc Human Factors Ergonomics Soc. 2013;57(1):1683-1687.

15. Rasche $P$, Schäfer $K$, Theis $S$, et al. Age-related usability investigation of an activity tracker. Int J Human Factors Ergon. 2016;4(3-4):187.

16. Puri A, Kim B, Nguyen $O$, et al. User acceptance of wrist-worn activity trackers among communitydwelling older adults: mixed method study. JMIR Mhealth Uhealth. 2017;5(11):e173.

17. Maastricht Instruments [Internet]. Maastricht (The Netherlands): Maastricht Instruments BV; 2019 [cited 2019 November 01]. Available from: http://www.accelerometry.eu/mox2/

18. Ummels D, Bijnens W, Aarts J, et al. The validation of a pocket worn activity tracker for step count and physical behaviour in older adults during simulated activities of daily living. 2020.

19. Alley S, Schoeppe S, Guertler D, et al. Interest and preferences for using advanced physical activity tracking devices: results of a national cross-sectional survey. BMJ Open. 2016;6(7):e011243.

20. Lebron J, Escalante K, Coppola J, et al. Long Island Systems, Applications and Technology Conference proceedings. Farmingdale (NY):; 2015.

21. Rosenberg D, Kadokura EA, Bouldin ED, et al. AMIA Annual Symposium proceedings. Chicago (IL): AMIA; 2016.

22. Shih PC, Han K, Poole ES, et al. iConference proceedings. Newport Beach (CA): IDEALS; 2015.

23. Armaǧan K, Çiǧdem E. Conference of Engineering Design proceedings. Milano (Italy): Politecnico di Milano, School of Design; 2015. 
24. Rabin C, Bock B. Desired features of smartphone applications promoting physical activity. Telemed J E Health. 2011;17(10):801-803.

25. Verwey R. Get moving! Self-management support using mobile technology [disseratation]. Maastricht (The Netherlands): Maastricht University; 2015.

26. Karapanos E, Gouveia R, Hassenzahl M, et al. Wellbeing in the making: peoples' experiences with wearable activity trackers. Psychol Well Being. 2016;6:4.

27. van der Weegen S, Verwey R, Spreeuwenberg $M$, et al. The development of a mobile monitoring and feedback tool to stimulate physical activity of people with a chronic disease in primary care: a usercentered design. JMIR Mhealth Uhealth. 2013;1(2):e8.

28. Project Catalyst [Internet]. Washington (DC): AARP Research; 2016 [cited 2020 June 18]. Available from: https://www.aarp.org/content/dam/aarp/home-and-family/personal-technology/2015-07/innovation50-project-catalyst-tracker-study-AARP.pdf

29. Tedesco S, Barton J, O'Flynn B. A review of activity trackers for senior citizens: research perspectives, commercial landscape and the role of the insurance industry. Sensors. 2017;17(6). DOI:10.3390/s17061277

30. Wang JB, Cadmus-Bertram LA, Natarajan L, et al. Wearable sensor/device (Fitbit One) and SMS textmessaging prompts to increase physical activity in overweight and obese adults: a randomized controlled trial. Telemed J E Health. 2015;21(10):782-792.

31. Schlomann A. A case study on older adults' long-term use of an activity tracker. Gerontechnology. 2017;16(2):115-124.

32. Fritz T, Huang EM, Murphy GC, et al. CHI proceedings. Vancouver (Canada): ACM; 2014.

33. Design Council [Internet]. London (UK): British Design Council; 2015 [2020 June 18]. Available from: https://www.designcouncil.org.uk/news-opinion/what-framework-innovation-design-councils-evolveddouble-diamond

34. Clegg D, Barker R. Case method fast-track: a RAD approach. Boston (MA): Addison-Wesley; 2004.

35. Maastricht Instruments [Internet]. Maastricht (The Netherlands): Maastricht Instruments BV; 2019 [2019 February 22]. Available from: https://www.accelerometry.eu/miss-activity/

36. Collen FM, Wade DT, Bradshaw CM. Mobility after stroke: reliability of measures of impairment and disability. Int Disabil Stud. 1990;12(1):6-9.

37. Elo S, Kyngas H. The qualitative content analysis process. J Adv Nurs. 2008;62(1):107-115.

38. Compendium of physical activities [Internet]. Tempe (AZ): Healthy Lifestyles Research Center, College of Nursing \& Health Innovation, Arizona State University; 2011 [cited 2020 October 26]. Available from: https://sites.google.com/site/compendiumofphysicalactivities/Activity-Categories/home-activity.

39. Health Council of the Netherlands [Internet]. Den Haag (The Netherlands): Health Council of the Netherlands; 2017 [cited 2018 January 20]. Available from: https://www.gezondheidsraad.nl/sites/ default/files/grpublication/beweegrichtlijnen_2017.pdf

40. Pharmacy [Internet]. Los Angeles (CA): The Noun Project; 2020 [cited 2020 June 18]. Available from: https://thenounproject.com/search/?q=pharmacy\&i=399860.

41. Help [Internet]. Los Angeles (CA): The Noun Project; 2020 [cited 2020 June 18]. Available from: https://thenounproject.com/search/?q=help\&i=2854287

42. App [Internet]. Los Angeles (CA): The Noun Project; [cited 2020 June 18]. Available from: https://thenounproject.com/search/?q=app\&i=1295151

43. Mobile Settings [Internet]. Los Angeles (CA): The Noun Project; [cited 2020 June 18]. Available from: https://thenounproject.com/search/?q=mobile\%20settings\&i=1018566

44. Privacy [Internet]. Los Angeles (CA): The Noun Project; [cited 2020 June 18]. Available from: https://thenounproject.com/search/?q=privacy\&i=2804339

45. Graph [Internet]. Los Angeles (CA): The Noun Project; [cited 2020 June 18]. Available from: https://thenounproject.com/search/?q=graphs\&i=2243582

46. Walking [Internte]. Los Angeles (CA): The Noun Project; [cited 2020 June 18]. Available from: https://thenounproject.com/search/?q=walking\&i=95239

47. Kujala S. User involvement: a review of the benefits and challenges. Behav Inform Technol. 2003;22(1): 1-16. 
48. Abras C, Maloney-Krichmar D, Preece J. Encyclopedia of human-computer interaction. Thousand Oaks (CA): Sage Publications; 2004.

49. National Institute for Public Health and the Environment [Internet]. Bilthoven (The Netherlands): National Institute for Public Health and the Environment; 2019 [cited 2019 June 21]. Available from: https://www.volksgezondheidenzorg.info/onderwerp/bevolking/cijfers-context/vergrijzing\#-node-totaalaantal-ouderen

50. Statistics Netherlands [Internet]. Heerlen (The Netherlands): Statistics Netherland; 2016 [cited 2020 June 18]. Available from: https://www.cbs.nl/nl-nl/nieuws/2016/52/75-plusserssterkst-groeiende-groepinternetters

51. Wouters M, Huygens M, Voogdt H, et al. E-health monitor: samen aan zet!. Utrecht (The Netherlands): Netherlands Institute for Health Services Research; 2019. 


\section{Appendix 4.1 Important characteristics to create a meaningful user-interface for elderly.}

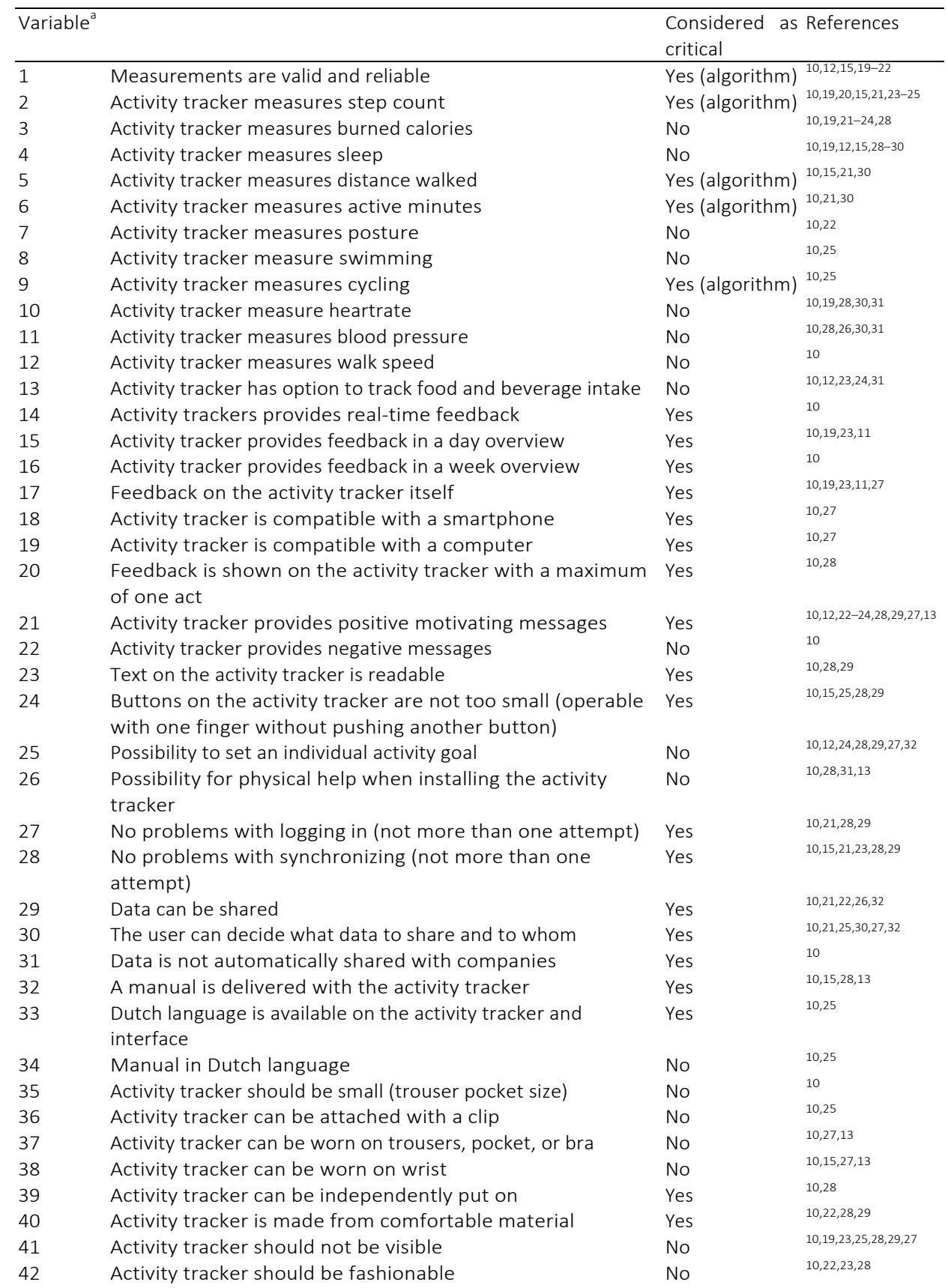




$\begin{array}{ll}\text { Yes } & 10 \\ \text { Yes } & 10,19,15 \\ \text { Yes } & 10,19,12,15,21,22,28,27 \\ \text { Yes } & 10,25,13\end{array}$
Activity tracker is waterproof Yes Activity tracker is damage proof (will not break after one Yes fall of 1.5-m height)

47 Activity tracker is compatible with iOs Yes

48 Activity tracker runs on current and new software version Yes of smartphones

$49 \quad$ Step count should be visible on the activity tracker

$10,23,27$

Yes 10
Interface uses graphs and symbols Activity tracker measures intensity of movements No Activity tracker measures walking stairs No

Yes 10

No 10

No 10,25

No 10,25

Activity tracker shows (on tracker or app) when the battery Yes 10,25 is almost empty

${ }^{a}$ Variables based on 48 interviews with people with a chronic disease ${ }^{10}$ (Chronic obstructive pulmonary disease (COPD), cardiovascular disease, chronic pain, oncology, osteoarthritis, and diabetes mellitus) who received treatment from a physiotherapist. Variables were propounded and confirmed with four experts in wearables. 


\section{Appendix 4.2. Questionnaire about the perceived effect of the MISS activity}

1. Overall, to what degree did you gain insight into your physical activity level?

1.1. o Very much more insight

1.2. o Much more insight

1.3. o A little more insight

1.4. o No change

1.5. o A little less insight

1.6. o Much less insight

1.7. o Very much less insight

2. To what degree did your physical activity level change?

2.1. o Very much more physical activity

2.2. o Much more physical activity

2.3. o A little more physical activity

2.4. o No change

2.5. o A little less physical activity

2.6. o Much less physical activity

2.7. o Very much less physical activity

3. To what degree was your physical activity more divided during the day?

3.1. o Very much more divided

3.2. o Much more divided

3.3. o A little more divided

3.4. o No change

3.5. o A little less divided

3.6. o Much less divided

3.7. o Very much less divided 


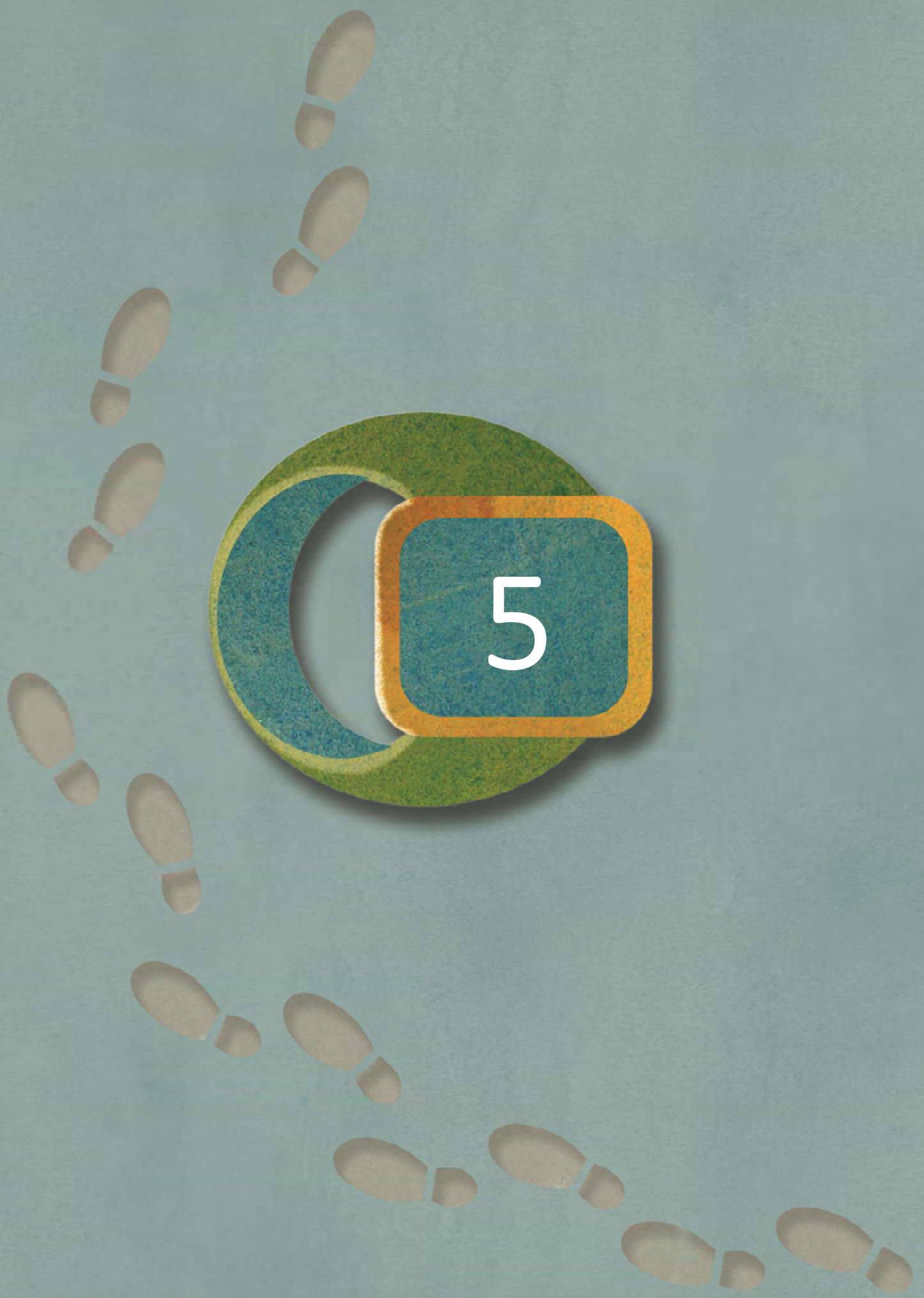




\section{Chapter}

The validation of a pocket worn activity tracker for step count and physical behavior in older adults during simulated activities of daily living

Darcy Ummels*, Wouter Bijnens*, Jos Aarts, Kenneth Meijer, Anna J. Beurskens, and

Emmylou Beekman 


\section{Abstract}

\section{Purpose}

The purpose of this study was to validate optimized algorithm parameter settings for step count and physical behavior for a pocket worn activity tracker in older adults during $A D L$. Secondly, for a more relevant interpretation of the results, the performance of the optimized algorithm was compared to three reference applications.

\section{Methods}

In a cross-sectional validation study, 20 older adults performed an activity protocol based on ADL with $\mathrm{MOX}_{\text {Missactivity }}$ versus $\mathrm{MOX}_{\text {Annegarn, }}$ activPAL, and Fitbit. The protocol was video recorded and analyzed for step count and dynamic, standing, and sedentary time. Validity was assessed by percentage error (PE), absolute percentage error (APE), Bland-Altman plots and correlation coefficients.

\section{Results}

For step count, the optimized algorithm had a mean APE of $9.3 \%$ and a correlation coefficient of 0.88 . The mean APE values of dynamic, standing, and sedentary time were $15.9 \%, 19.9 \%$, and $9.6 \%$, respectively. The correlation coefficients were $0.55,0.91$, and 0.92, respectively. Three reference applications showed higher errors and lower correlations for all outcome variables.

\section{Conclusion}

This study showed that the optimized algorithm parameter settings can more validly estimate step count and physical behavior in older adults wearing an activity tracker in the trouser pocket during ADL compared to reference applications. 


\section{Introduction}

In the past decade, activity trackers have been used more frequently by a relatively young and physically active population. ${ }^{1}$ In addition to this population, activity trackers can also be beneficial for older adults (65+). In 2018, only $37 \%$ of the older adults in the Netherlands were sufficiently physically active according to Dutch guidelines. ${ }^{2}$ Activity trackers can contribute to overcome this by giving insight into the amount of physical activity, increasing awareness and motivating older adults to be more physically active. ${ }^{3-8}$ Several studies have shown that older adults are most interested in step count and amount of physical behavior as outcome variables for physical activity. ${ }^{3,8-10}$ Recent studies have shown that step count and physical behavior are not validly measured by consumer-grade activity trackers during low walking speeds, which often occur during activities of daily living (ADL) such as household activities. ${ }^{11-21}$ This lower validity can partly be explained by the fact that the majority of consumer-grade activity trackers don't have older adults as a target group and don't adjust their algorithms accordingly.

Recently, an adjustable classification algorithm was published to optimize algorithm performance. ${ }^{22}$ Through easily adjustable algorithm parameters it is possible to optimize the performance of this algorithm for different target and tracker wear locations. A recent qualitative study showed that older adults would prefer to wear an activity tracker in their trouser pocket. ${ }^{8}$ Consequently, the adjustable algorithm was optimized to estimate step count and dynamic, standing, and sedentary time for older adults and a pocket worn activity tracker according to the proposed method by. ${ }^{22}$

The first purpose of this study was to validate these optimized algorithm parameter settings for step count and physical behavior expressed as dynamic, standing, and sedentary time in older adults with a normal pattern wearing an activity tracker in their trouser pocket during simulated ADL. Secondly, to have a more relevant interpretation of the validation results, the performance of the optimized algorithm parameter settings for older adults was compared to the algorithm where the adjustable classification algorithm originates from and two frequently used activity trackers.

\section{Methods}

\section{Study design}

A cross-sectional validation study was performed in which the optimized algorithm parameter settings were validated and compared to the algorithm where the adjustable classification algorithm originates from and two activity trackers. 


\section{Participants}

Twenty older adults were recruited from several local associations for older adults (e.g., bridge club or church association) in the South of the Netherlands. Participants were included if they were older than 65 years and didn't meet the Dutch physical activity guidelines (a minimum of 150 min of moderate-intensity per week). ${ }^{23}$ Exclusion criteria were insufficient understanding of the Dutch language, use of a walking aid, and a pathological gait during the 10-metre walk test (10MWT) observed by a physiotherapist. ${ }^{24}$ All participants provided written informed consent prior to inclusion.

\section{Activity protocol}

A participant-determined sequence activity protocol was developed based on ADL. To simulate free-living, participants were free to choose the order and duration of a series of daily activities. The activity protocol, shown in Table 5.1, was based on earlier activity protocols with ADL in people with chronic diseases and older adults. ${ }^{20,25-28}$

Table 5.1 The Participant-Determined Sequence Activity Protocol with Activities of Daily Living for Older adults.

\begin{tabular}{ll}
\hline Activity type $^{a}$ & Defined for the gold standard as \\
\hline Squat $^{a}$ & Marks start of the protocol \\
Organising a cabinet with cutlery, plates, and cups & Standing \\
Reading the newspaper while seated at a table & Stedentary \\
Ironing and folding laundry & Standing \\
Sitting and talking & Sedentary \\
Washing the dishes & Standing \\
Sweeping the floor & Dynamic \\
Changing linens on a bed $_{\text {Setting the table with cutlery, plates, and cups }}$ & Dynamic \\
Squat $^{\text {a }}$ & Dynamic \\
\hline
\end{tabular}

a Squat was mandatory at the beginning and at the end of the activity protocol and was not used for analysis.

\section{Activity trackers}

The MOX Activity Logger ${ }^{29}$ contains a tri-axial accelerometer (ADXL362, Analog Devices, Norwood, MA, US). This small, lightweight, waterproof device (35 mm x $35 \mathrm{~mm}$ x $10 \mathrm{~mm}$, $11 \mathrm{~g})$ measures raw acceleration data $( \pm 8 \mathrm{~g})$ in three orthogonal sensor axes $(X, Y$, and $Z)$ at a $25 \mathrm{~Hz}$ sampling rate. The raw data is stored directly on the internal memory. The MOX has storage capacity and battery life for continuous measurements up to 7 days. Device configuration, data transfer and charging of the device are possible via an USB connection. Data analysis is performed offline. The MOX was worn in the front trouser 
pocket, attached with a clip, to secure a fixed orientation of the device with respect to axial mobility of the upper leg. This wear location is shown in Figure 5.1.

Two commonly used activity trackers, the activPAL ${ }^{30}$ and the Fitbit Alta HR (Fitbit; Fitbit Inc., San Fransisco, CA, US) were used as reference for a more relevant interpretation of the validation results. Based on the recommendations of the manufactures the activ-PAL is worn on the dominant leg ten centimetres above the patella (activPAL) and the Fitbit is worn on the nondominant wrist. ${ }^{31}$

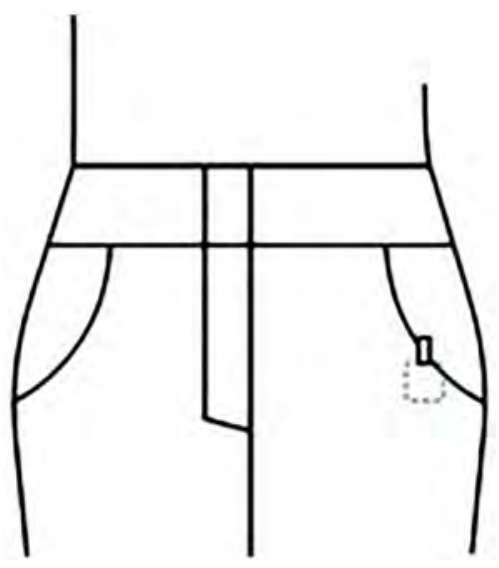

Figure 5.1 MOX wear location.

\section{Data collection and procedure}

Participants were measured at the Human Performance Laboratory of Maastricht University (Maastricht, NL) or at Zuyd University of Applied Science (Heerlen, NL). Both laboratories are comparable in size (about $120 \mathrm{~m}^{2}$ ) and facilities. Demographic data were collected (gender, age, body weight, and body length) by two researchers, either DU (physiotherapist) or WB (application engineer). Thereafter, the participants performed the $10 \mathrm{MWT}$ to calculate their average walking speed. After the $10 \mathrm{MWT}$, participants were fitted with the MOX, the activPAL, and the Fitbit. The same MOX, activPAL, and Fitbit were used for all participants.

The activity protocol was recorded on video and observed to use as a gold standard to determine the actual step count, dynamic, standing, and sedentary time in seconds performed by the participants. Step count was counted manually by two independent observers using the counter application Counter+. ${ }^{32}$ A step was defined as: "when the entire foot is lifted from the floor and when the participants replaced their foot (forward, backward, sideways or upwards)". ${ }^{33}$ After manually counting the step count, 
the video was re-observed and the time that the participants performed dynamic (walking and walking during household activities), standing or sedentary (sitting, lying) time was noted. Physical behavior was assessed by two independent observers (Table 5.1) using the EasyTag app. ${ }^{34}$

The data from the activity trackers were collected directly after the activity protocol. Analysis of the raw acceleration data of the MOX took place on a PC after the measurements (off-line) using Matlab (R2018b, The MathWorks Inc., Natick, MA, US) with two algorithms. The first one is the activity classification algorithm presented and validated by $^{35}$ for healthy adults (MOXAnnegarn), where the adjustable classification algorithm originates from. The second one is the classification algorithm with application specific adjustable parameters itself. ${ }^{22}$ For application in an older adult target group wearing an activity tracker in their trouser pocket the optimized parameter settings are: a data segmentation window size of $2 \mathrm{~s}$, an amount of physical activity threshold of five counts per second (cps) and an orientation threshold of $0.8 \mathrm{~g}$. This application is referred to as Miss Activity, the parameter settings as MOXMissActivity. For MOXAnnegarn dynamic, standing, and sedentary time spent in seconds were retrieved. In addition to these three variables, for MOXMissactivity step count was also retrieved. For the activPAL, step count and dynamic, standing, and sedentary time spent in seconds were retrieved from the PAL Software Suite (v7.2.32; PAL Technologies Ltd., Glasgow, Scotland, UK). For the Fitbit, step count and active minutes (by definition: ten continuous minutes long bouts of moderate-to intense activity $>3$ metabolic equivalent of task [MET] $)^{36}$, were retrieved from the corresponding Fitbit app (Fitbit Inc., San Fransisco, CA, US). From this point, we refer to the active minutes of the Fitbit as dynamic time.

\section{Data analysis}

Data analysis was performed using SPSS Statistics (version 23.0; IBM Corp, Armonk, NY, US) and Prism (GraphPad Prism 8.2.1(441); GraphPad Software, San Diego, CA, USA).

Descriptive statistics of the participant characteristics were presented as a number (percentage) for the categorical variable gender and as a mean (95\% confidence interval [Cl]) for the continuous variables age, body length, body weight, and average walk speed.

\section{Inter-observer reliability of the video observations}

The differences in step count, dynamic, standing, and sedentary time was calculated between two observers. If there was more than a $5 \%$ difference between the two observers, a third observer assessed the video. The inter-observer reliability of the two observers with the smallest difference was assessed by an Intraclass Correlation 
Coefficient (absolute agreement, two-way random) and Bland-Altman plots with limits of agreement. It was hypothesized that there was a strong correlation between observers $(r \geq 0.90)$ in order to guarantee a robust gold standard. ${ }^{37}$

\section{Validation}

To check for outliers in the data of MOX Miss-Activity, MOX Annegarn, activPAL, Fitbit versus the video observations regarding the variables step count, dynamic, standing, and sedentary time the data were transformed to z-scores and Bland-Altman plots were visually inspected. In case of outliers, pairwise deletion was applied.

For step count, dynamic, standing, and sedentary time the mean $(95 \% \mathrm{Cl})$, mean difference, the percentage error (PE), the absolute percentage error (APE), and the smallest detectable change (SDC) were used to gain insight into the algorithm's and activity trackers' performance compared to video observations.

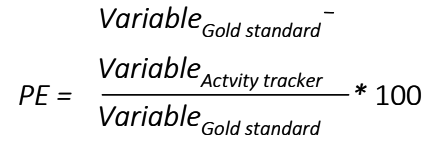

$$
\begin{aligned}
& A P E=\frac{\left|\begin{array}{l}
\text { Variable }_{\text {Gold standard }}- \\
\text { Variable }_{\text {Activity tracker }}
\end{array}\right|}{\text { Variable }_{\text {Gold standard }}} * 100 \\
& S D C=1.96 * \sqrt{2} * \text { standard error measurement }
\end{aligned}
$$

Formula 1 and 2 show the calculation of PE and APE for each variable. A PE or APE of less than $10 \%$ was considered acceptable. ${ }^{38}$ Formula 3 shows the calculation of SDC.

The level of agreement between step count, dynamic, standing, and sedentary time and the video observations were examined by a Bland-Altman plot with their limits of agreement. ${ }^{39}$ Pearson correlation coefficients were calculated to gain insight into the relationship between the $\mathrm{MOX}_{\text {Missactivity, }}$ MOX Annegarn, activPAL, Fitbit versus the video observations regarding the variables step count, dynamic, standing, and sedentary time. It was hypothesised that there would be at least a substantial correlation $(r \geq 0.60){ }^{37} \mathrm{~A}$ paired sample $t$-test was used to determine large systematic differences between the MOX $_{\text {MissActivity, }}$ MOX $_{\text {Annegarn, }}$ activPAL, Fitbit versus the video observations regarding the variables step count, dynamic, standing, and sedentary time. A p-value below 0.05 was considered to be statistically significant. Additionally the sensitivity, specificity and accuracy are calculated. 


\section{Results}

\section{Participant Characteristics}

Twenty older adults were recruited for this study. The participant characteristics are displayed in Table 5.2.

Table 5.2 Participant characteristics.

\begin{tabular}{lc}
\hline Characteristic & Participants $(n=20)$ \\
\hline Gender, male, $n(\%)$ & $10(50 \%)$ \\
Age, years, mean $(95 \% \mathrm{Cl})$ & $74.5(70.9-77.6)$ \\
Body weight, kilograms, mean $(95 \% \mathrm{Cl})$ a & $86.1(73.7-98.5)$ \\
Body length, centimetres, mean $(95 \% \mathrm{Cl})$ & $172.5(167.6-176.9)$ \\
Average walk speed, mean $(95 \% \mathrm{Cl})$ & $1.1(1.0-1.2)$ \\
\hline
\end{tabular}

aThere was one (5\%) missing value for body weight.

\section{Inter-Observer Reliability of the Video Observations}

The inter-observer reliability of the video observations calculated for step count was high (ICCagreement 0.98, $P<0.00195 \% \mathrm{Cl} 0.95-0.99$ ). The inter-observer reliability of dynamic, standing, and sedentary time were also high (ICCagreement $0.98, P<0.001$, 95\% Cl 0.95-0.99), (ICCagreement 0.99, $P<0.001$ 95\% Cl 0.98-0.99), (ICCagreement, 1.0, $P<0.001,95 \% \mathrm{Cl} 0.99-1.0)$ respectively. The limits of agreement for step count ( -58 to 62 steps), dynamic ( -49 to $41 \mathrm{~s}$ ), standing ( -49 to $40 \mathrm{~s}$ ) and sedentary time ( -26 to $28 \mathrm{~s}$ ) showed no systematic differences. A third observer had to be included in two cases.

\section{Step count}

Descriptive statistics for each activity tracker are shown in Table 5.3. The mean step count during the activity protocol counted by the video observation was 615 (566-664) steps. The MOX MissActivity had a mean step count of 602 (537-667) steps, the activPAL had a mean step count of 385 (336-433) steps and the Fitbit had a mean step count of 731 (590-873) steps. The values of the percentage error and the absolute percentage error are presented in Figure 5.2. The Bland-Altman plots (Figure 5.3) show a slight overestimation of the number of steps in the MOX MissActivity, an overestimation in the activPAL and underestimation in the Fitbit. If the limits of agreement for the activPAL and Fitbit Alta HR are corrected for their respective bias they are -141 to 140 and -183 to 183 , respectively. 


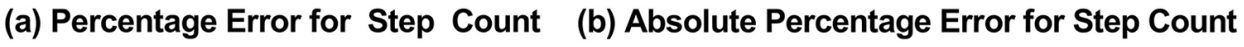
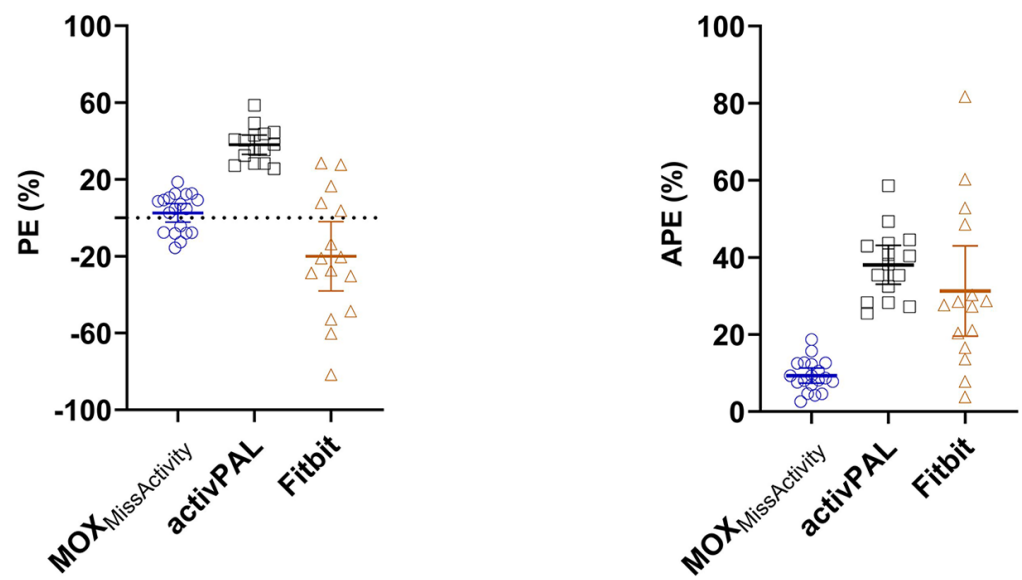

Figure 5.2 (a) Percentage error and (b) absolute percentage error for step count. Step count for the MOXMissActivity is presented in blue, for activPAL in black and for Fitbit in brown.

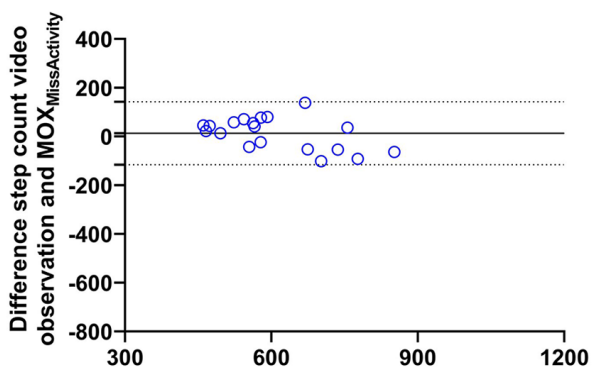

(a) Mean step count video observation and MOX MissActivity

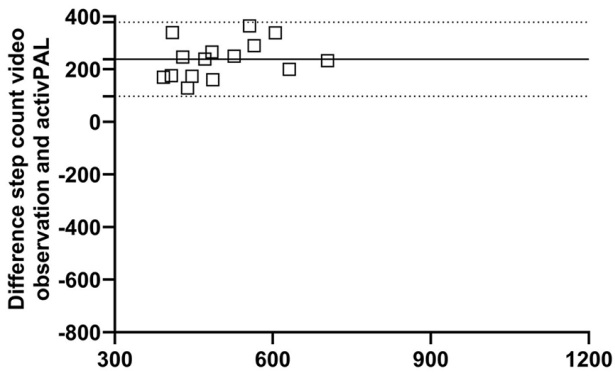

(b) Mean step count video observation and activPAL

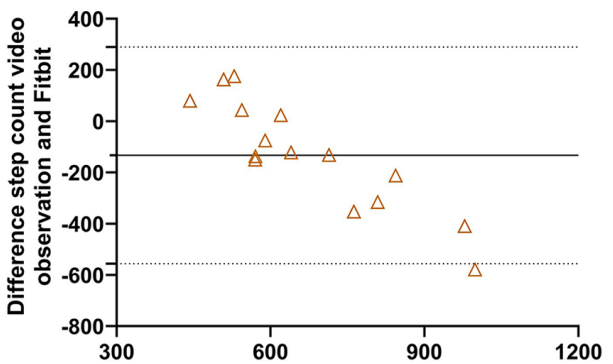

(c) Mean step count video observation and Fitbit

Figure 5.3 Bland-Altman plots of the (a) MOXMissActivity, (b) activPAL, and (c) the Fitbit versus the video observation. 


\section{Physical behavior}

The mean dynamic, standing, and sedentary time during the activity protocol counted by the video observations were 422 (387-457), 668 (579-757), and 1716 (1452-1981) seconds, respectively. On average the total protocol lasted 45 (40-51) min. For the

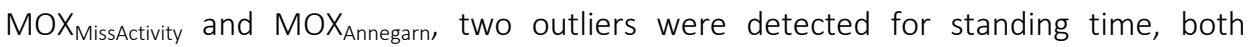
outliers were visible in the Bland-Altman plots. The video recordings were re-watched and, in both participants, the MOX was incorrectly worn. One of these outliers was also statistically detected with the z-score ( $5 \%$ with a $z$-score of $>2.58$ ). One outlier for the activPAL was detected for dynamic time, the outlier was visible in the Bland-Altman plot and was detected with the $z$-score ( $5 \%$ with a z-score of $>3.29$ ).

The mean dynamic, standing, and sedentary time for the MOX Missactivity without outliers was 405 (338-473), 696 (504-889), and 1692 (1383-2001) seconds, respectively. For the MOXAnnegarn the mean dynamic, standing, and sedentary time was 152 (131-174), 927 (712-1141), and 1715 (1392-2038), respectively. For the activPAL, the mean dynamic, standing, and sedentary time was 309 (255-364), 1256 (859-1645), and 1234 (946-1522) seconds respectively. The mean dynamic time for the Fitbit was 1 (0-3) minutes.

Descriptive statistics without outliers for each activity tracker are shown in Table 5.4 and the descriptive statistics with outliers for each activity tracker are shown in Supplementary Table S5.1. The PE and APE for physical behavior are shown in Figure 5.4. To gain more insight in validity, not only based on total time but also on window-towindow basis, additional analyzes in terms of specificity, sensitivity, and accuracy were performed (Supplementary Table S5.2).

The Bland-Altman plots of the MOXMissActivity without outliers (Figure 5.5) show a slight overestimation for dynamic and sedentary time and a slight underestimation for standing time. When the limits of agreement for the activPAL are corrected for their bias for dynamic, standing, and sedentary time, they are -141 to $147,-538$ to 527 and -1234 to 1130 respectively. 

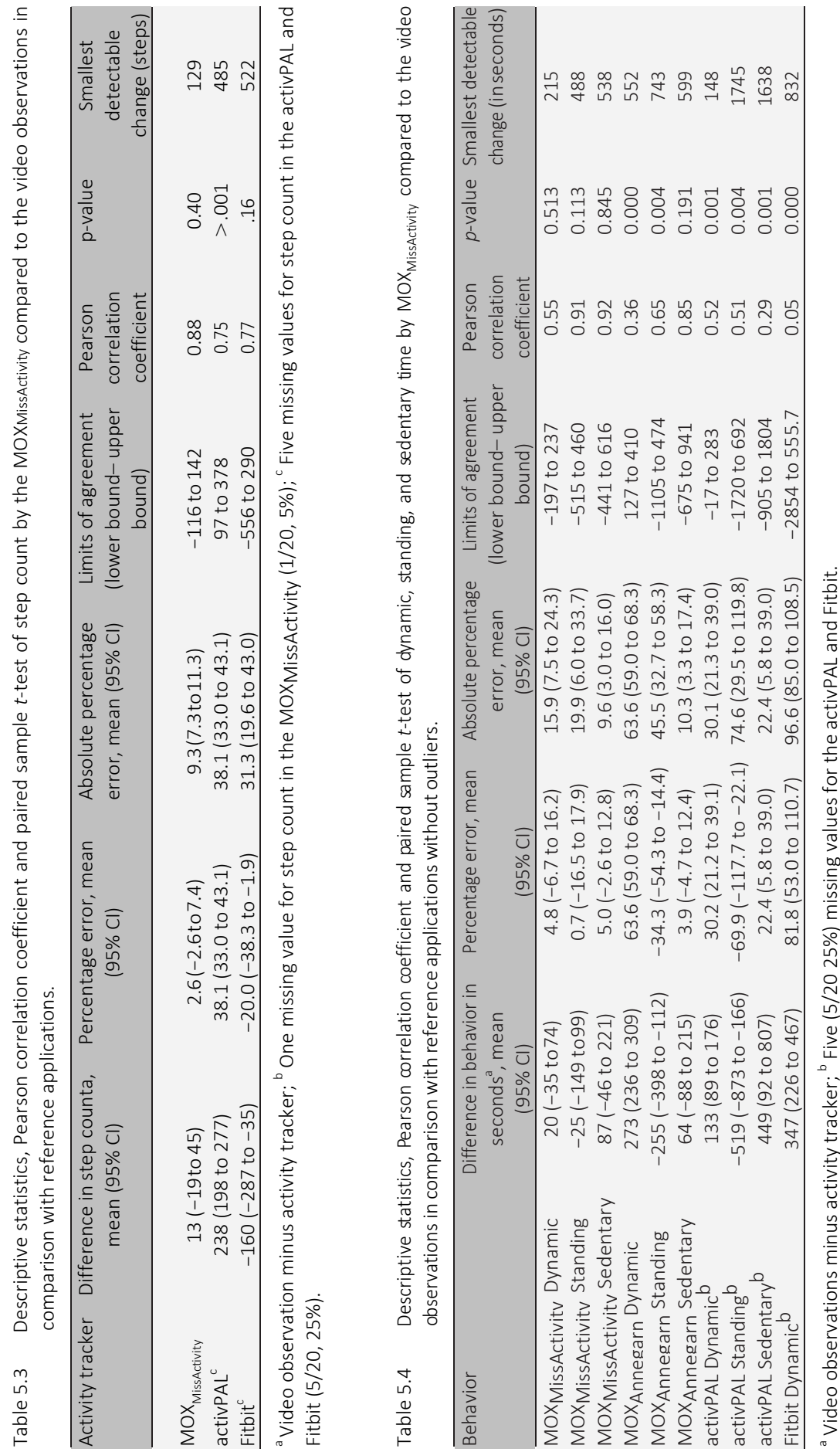
(a) Percentage Error for Physical Behaviour

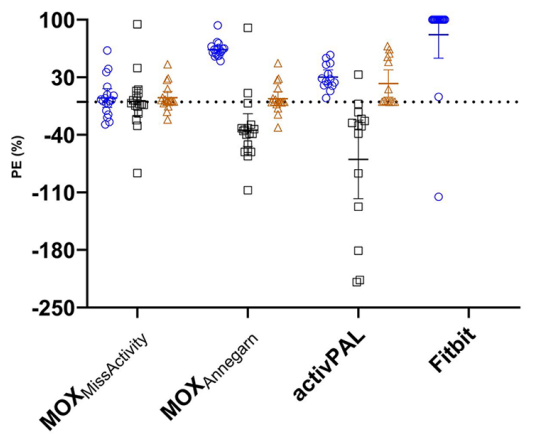

(b) Absolute Percentage Error for Physical Behaviour

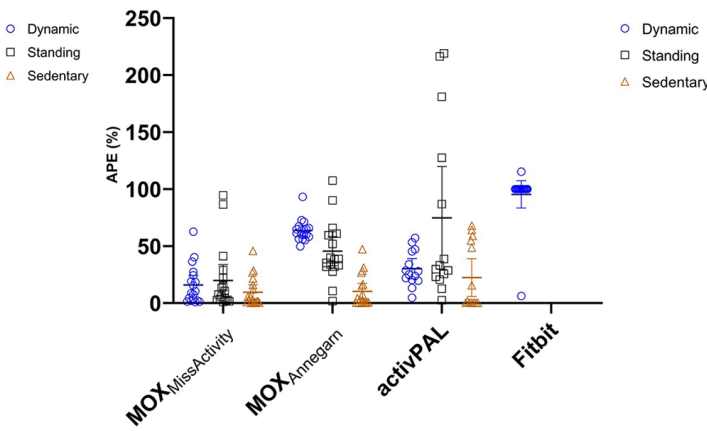

Figure 5.4 (a) Percentage Error and (b) Absolute Percentage Error for dynamic, standing, and sedentary time. PE and APE for dynamic time are presented in blue, for standing time in black and for sedentary time in brown. The Fitbit Alta HR measures dynamic time only, therefore no data for standing and sedentary time are presented.

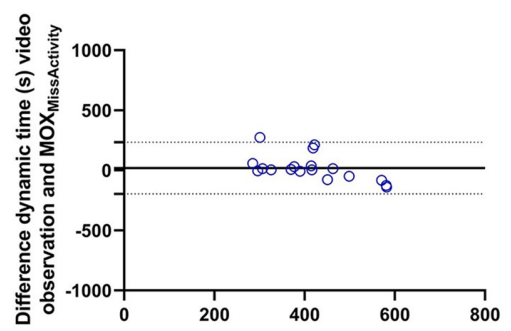

(a) Mean dynamic time (s) video observation and MOX MissActivity $_{\text {}}$

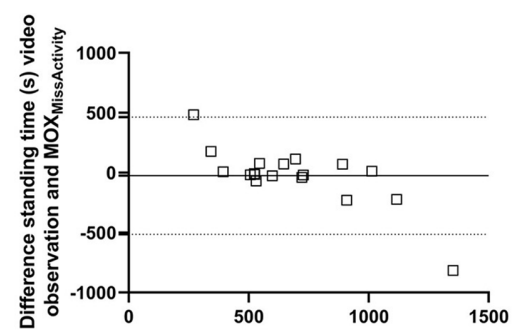

(b) Mean standing time (s) video observation and MOX MissActivity $_{\text {. }}$

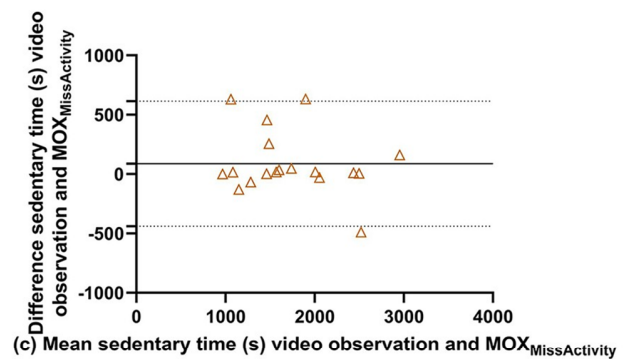

Figure 5.5 (a) Bland- Altman plots of dynamic, (b) standing, and (c) sedentary time of the MOX MissActivity $_{\text {without }}$ outliers versus the video observations. 


\section{Discussion}

\section{Principal findings}

This study showed that the optimized algorithm parameter settings (MOX Missactivity) can more validly measure step count and physical behavior expressed as dynamic, standing, and sedentary time in older adults wearing an activity tracker in their trouser pocket during $A D L$ based on a test combination of PE, APE, correlation coefficients, and paired sample $t$-test compared to the MOXAnnegarn, activPAL, and Fitbit.

The variables step count and sedentary time showed good validity in comparison with the gold standard. It was hypothesised that all variables of the MOXMissactivity would have at least a substantial correlation $(r \geq 0.60)$ and an APE of $<10 \%$. Only the variable dynamic time had a slightly lower correlation coefficient of 0.55 and the variables dynamic and standing time had a mean APE of $15.9 \%$ and $19.9 \%$. These results are supported with additional analyzes of the specificity, sensitivity, and accuracy (Supplementary Table S5.2). This can be explained by the fact that the activities in the activity protocol were classified into dynamic, standing, and sedentary behavior as a whole. For example, by definition, the video observations classified changing linens as dynamic behavior, however during this activity it is possible that the participant performed a sequence of standing and dynamic behavior (e.g., standing still to put the cushion on the bed).

In the current study, MOXAnnegarn, activPAL, and Fitbit showed lower validity compared to the gold standard and the MOXMissactivity. It is clear that these target group and wear location specific classification algorithms cannot be applied outside of their specific context. The results for the activPAL are in line with a recent study showing a low validity during short stepping bouts and activities with low walking speeds, such as shuffling, picking, transitions, and kneeling in older adults. ${ }^{40,41}$ The underestimation for dynamic time of the Fitbit can be explained by the definition Fitbit uses for active minutes: 10 continuous minutes long bouts of moderate-to intense activity $>3 \mathrm{MET}$. It is reasonable to assume that activities of daily living weren't performed with such intensity and/or for that long during this protocol.

\section{Limitations and strengths}

This study had some limitations, the first one being the relatively low sample size $(n=20)$. Future work could include a larger sample size, although several validity studies have been performed with a sample size of $20 .{ }^{14}$

Second, that due to some technical errors, there were five missing values for both the Fitbit and the activPAL. However, since these activity trackers were only used as reference this should not have affected the main purpose of the current study. Third, 
the varying MOX wear location relative to the body. To secure the validity of the algorithm the MOX should always be correctly placed in the trouser pocket below the waist, this should be addressed in a manual. By re-observing the video recordings, it was noted that the MOX was placed above the participants' hip in two cases. Since the algorithm assumes a wear location on the upper leg, the MOX was not able to correctly measure within these two participants due to this misplacement. Therefore, it was chosen to handle these two cases as outliers, since the misplacement, and not the algorithm, compromised the validity. Fourth, this study was performed in a lab setting and is therefore not directly generalizable to daily life. However, with the ADL protocol, daily life was simulated as close as possible to daily life. This is in line with the proposed standardization methods of Welk et al. ${ }^{42}$

A strength of this study is the use of the participant-determined sequence activity protocol to validate the algorithm. This activity protocol simulates free-living since participants were free to choose the order and duration of the activities they performed. To simulate the free-living situation as best as possible activities that are frequently performed by older adults are included in the activity protocol. Furthermore, this study follows the recommendations made by Welk et al. ${ }^{42}$ for validation studies in wearables: use a diverse sample, appropriate sampling of daily behavior, an appropriate criterion measure, standardised protocols and wear locations, and inclusion of reference applications. To standardise the analyzes they recommend to use relevant metrics, documenting the error and the direction of the error and to focus on equivalence. ${ }^{42}$ Another strength of this study is the high inter-observer reliability resulting in a robust gold standard (range $r=0.96-1.0$ ).

\section{Clinical implications}

From previous research it is known that consumer-grade activity trackers can't measure step count and physical behavior validly during low walking speeds, which often occurs in older adults and during ADL. ${ }^{11-21}$ Apparently, daily life of older adults differs that much from the target group of these consumer-grade activity trackers that their algorithms are not sufficient for older adults. Therefore, it is important to have an algorithm optimized for the target group, wear location and their specific activities. If a consumer-grade activity tracker is used for this target group, the algorithm should ideally be personalised to the specific target group or at least bias corrections to the outcomes of the algorithm should be applied. The validity of the optimized algorithm is limited to older adults with a normal gait pattern. This study shows that an optimized algorithm is indeed more valid than general purpose activity trackers. As is shown by the smallest detectable change the optimized algorithm could also detect change in patient's physical activity level 
sooner. However, this study is performed on a group level and not on an individual level. Therefore, the interpretation on an individual level must be performed carefully since the optimized algorithm can both over- and underestimate step count and physical behavior depending on the number of steps or seconds.

For an activity tracker to be useful in daily life, validity is important, but feasibility is equally important. In a future feasibility study, development of a user-friendly userinterface of the MISS Activity will be addressed. The validated algorithm together with the user-interface will be called the Measure It Super Simple (MISS) Activity. ${ }^{43}$

\section{Conclusion}

This study showed that the optimized algorithm parameter settings can more validly estimate step count, dynamic, standing, and sedentary time in older adults with a normal gait pattern wearing an activity tracker in their trouser pocket during a participant-determined sequence activity protocol with activities of daily living compared to reference applications with generic activity tracker algorithms. For future studies and clinical practice an algorithm should ideally be optimized to the target population. Future work will include the development of a target group-specific userfriendly application.

\section{Acknowledgments}

We would like to thank Joey Gerritsen, Henk Marell, Harry Bakels and all participants who participated in this study. We thank Christopher McCrum for proofreading and editing an earlier version of the manuscript. 


\section{References}

1. Macridis S, Johnston N, Johnson S, Vallance JK. Consumer physical activity tracking device ownership and use among a population-based sample of adults. PLoS One 2018;13(1):e0189298.

2. National Institute for Public Health and the Environment. Beweeggedrag bij personen van 4 jaar en ouder in 2018. National Instuitue for Public Health. https://www.rivm.nl/leefstijlmonitor/bewegen. 2019.

3. Maher C, Ryan J, Ambrosi C, Edney S. Users' experiences of wearable activity trackers: a cross-sectional study. BMC Public Health 2017;17(1):880.

4. Mercer K, Giangregorio L, Schneider E, Chilana P, Li M, Grindrod K. Acceptance of commercially available wearable activity trackers among adults aged over 50 and with chronic illness: A mixed-methods evaluation. JMIR Mhealth Uhealth 2016;4(1):e7.

5. O'Brien T, Troutman-Jordan M, Hathaway D, Armstrong S, Moore M. Acceptability of wristband activity trackers among community dwelling older adults. Geriatric Nursing 2015;36(Suppl. 2):S21-S25.

6. Preusse KC, Mitzner TL, Fausset CB, Rogers WA. Older adults' acceptance of activity trackers. J Appl Gerontol 2017;36(2):127-155.

7. Sullivan AN, Lachman ME. Behavior change with fitness technology in sedentary adults: A review of the evidence for increasing physical activity. Front Public Health 2016;4:289.

8. Ummels D, Beekman E, Moser A, Braun SM, Beurskens AJ. Patients' experiences with commercially available activity trackers embedded in physiotherapy treatment: A qualitative study. Disabil Rehabil 2020;42(23):3284-3292

9. Rosenberg D, Kadokura EA, Bouldin ED, Miyawaki CE, Higano CS, Hartzler AL. Acceptability of Fitbit for physical activity tracking within clinical care among men with prostate cancer.AMIA Annu Symp Proc 2017;2016:1050-1059.

10. Schlomann A. A case study on older adults' long-term use of an activity tracker. Gerontechnology 2017;16(2):115-124.

11. Alharbi M, Bauman A, Neubeck L, Gallagher R. (2016). Validation of Fitbit-Flex as a measure of freeliving physical activity in a community-based phase III cardiac rehabilitation population. Eur J Prev Cardiol 2016; 23(14):1476-1485.

12. Beevi FH, Miranda J, Pedersen CF, Wagner S. An evaluation of commercial pedometers for monitoring slow walking speed populations. Telemed J E Health 2016;22(5):441-449.

13. Cyarto EV, Myers A, Tudor-Locke C. Pedometer accuracy in nursing home and communitydwelling older adults. Med Sci Sports Exerc 2004;36(2):205-209.

14. Evenson KR, Goto MM, Furberg RD. Systematic review of the validity and reliability of consumer-wearable activity trackers. Int J Behav Nutr Phys Act 2015;12:159.

15. Ferguson $\mathrm{T}$, Rowlands $\mathrm{AV}$, Olds $\mathrm{T}$, Maher $\mathrm{C}$. The validity of consumer-level, activity monitors in healthy adults worn in free-living conditions: a cross-sectional study. Int J Behav Nutr Phys Act 2015;12(1):42.

16. Floegel TA, Florez-Pregonero A, Hekler EB, Buman MP. Validation of consumer-based hip and wrist activity monitors in older adults with varied ambulatory abilities. The Journals of Gerontology Series A: Biological Sciences and Medical Sciences, 2016;72(2):229-236. 8

17. Martin JB, Krč KM, Mitchell EA, Eng JJ, Noble JW. Pedometer accuracy in slow walking older adults. Int J Ther Rehabil 2012;19(7):387-393.

18. Straiton N, Alharbi M, Bauman A, Neubeck L, Gullick J, Bhindi R, Gallagher R. (2018). The validity and reliability of consumer-grade activity trackers in older, community-dwelling adults: A systematic review. Maturitas 2018;112:85-93.

19. Tedesco S, Sica M, Ancillo A, Timmons S, Barton J, O'Flynn B. Accuracy of consumer-level and researchgrade activity trackers in ambulatory settings in older adults. PLoS One 2019;14(5):e0216891.

20. Ummel, D, Beekman E, Theunissen K, Braun S, Beurskens AJ. (2018). Counting steps in activities of daily living in people with a chronic disease using nine commercially available fitness trackers: Cross-sectional validity study. JMIR Mhealth Uhealth 2018;6(4):e70.

21. Van Blarigan EL, Kenfield SA, Tantum L, Cadmus-Bertram LA, Carroll PR, Chan JM. The Fitbit one physical activity tracker in men with prostate cancer: Validation study. JMIR Cancer 2017;3(1):e5.

22. Bijnens W, Aarts J, Stevens A, Ummels D, Meijer K. Optimization and validation of an adjustable activity classification algorithm for assessment of physical behavior in elderly. Sensors 2019;19(24):5344. 
23. Health Council of the Netherlands. Beweegrichtlijnen 2017. Independent scientific advisory body for government and parliament. https://www.gezondheidsraad. nl/documenten/adviezen/ 2017/08/22/beweegrichtlijnen-2017. 2017.

24. Collen FM, Wade DT, Bradshaw CM. Mobility after stroke: Reliability of measures of impairment and disability. International Disability Studies 1990;12(1):6-9.

25. Cavalheri V, Donaria L, Ferreira T, Finatti M, Camillo CA, Cipulo Ramos EM, Pitta F. Energy expenditure during daily activities as measured by two motion sensors in patients with COPD. Respiratory Medicine 2011;105(6):922-929.

26. Erasmus MC University Medical Center Rotterdam. (2013). Validation of the active8 activity monitor: Detection of body postures and movements. https://www.activ8all.com/front/wp-content/uploads/ 2014/08/ReportActiv8_EMC.pdf

27. Langer D, Gosselink R, Sena R, Burtin C, Decramer M, Troosters T. Validation of two activity monitors in patients with COPD. Thorax 2009;64(7):641-642.

28. Sant'Anna T, Escobar VC, Fontana AD, Camillo CA, Hernandes NA, Pitta F. (2012). Evaluation of a new motion sensor in patients with chronic obstructive pulmonary disease. Arch Physical Med Rehabil 2012;93(12):2319-2325.

29. Maastricht Instruments BVa. (2020) MOX1. Accelerometry. https://www.accelerometry.eu/mox1/

30. activPAL. activPAL operating guide.

31. Fitbit.(2020a). Fitbit Alta introductory guide. https://www.fitbit.com/nl/alta/alta-101

32. Seedform. (2020). Counter plus. Google Play Store. https://play.google.com/store/apps/details?id= com.seedform.counter\&hl=nl

33. Beekman E, Braun SM, Ummels D, van Vijven K, Moser A, Beurskens AJ. (2017). Validity, reliability and feasibility of commercially available activity trackers in physical therapy for people with a chronic disease: A study protocol of a mixed methods research. Pilot and Feasibility Studies 2017;3(1):1-10.

34. Dartfish Ltd.. Dartfish easytag-note. Google Play. https://play.google.com/store/apps/details?id= com.dartfish.android.tag\&hl=nl. 2020.

35. Annegarn J, Spruit MA, Uszko-Lencer NH, Vanbelle S, Savelberg HH, Schols AM, Meijer K. Objective physical activity assessment in patients with chronic organ failure: a validation study of a new single-unit activity monitor. Arch Physical Med Rehabil 2011;92(11):1852-1857.e1.

36. Fitbit. (2020b). What are active minutes? https://help.fitbit.com/articles/en_US/Help_article/1379

37. De Vet HC, Terwee CB, Mokkink LB, Knol DL. Measurement in medicine: A practical guide. Cambridge University Press. 2011.

38. Sasaki JE, Hickey AM, Staudenmayer JW, John D, Kent JA, Freedson PS. Performance of activity classification algorithms in free-living older adults. Medicine \& Science in Sports \& Exercise 2016;48(5), 941-950.

39. Bland JM, Altman DG. Statistical methods for assessing agreement between two methods of clinical measurement. Lancet, 1986;8(1):307-310.

40. Bourke AK, Ihlen EAF, Helbostad JL. Validation of the activPAL3 in free-living and laboratory scenarios for the measurement of physical activity, stepping, and transitions in older adults. Journal for the Measurement of Physical Behaviour 2019;2(2):58-65.

41. Feehan LM, Geldman J, Sayre EC, Park C, Ezzat AM, Yoo JY, Li LC. Accuracy of Fitbit devices: Systematic review and narrative syntheses of quantitative data. JMIR Mhealth Uhealth 2018;6(8), e10527.

42. Welk GJ, Bai Y, Lee JM, Godino J, Saint-Maurice PF, Carr L. Standardizing analytic methods and reporting in activity monitor validation studies. Medicine and Science in Sports and Exercise 2019;51(8): 1767-1780.

43. Maastricht Instruments BVb. MISS activity. Accelerometry. https://www.accelerometry.eu/miss-activity/, 2020. 
Supplemental material

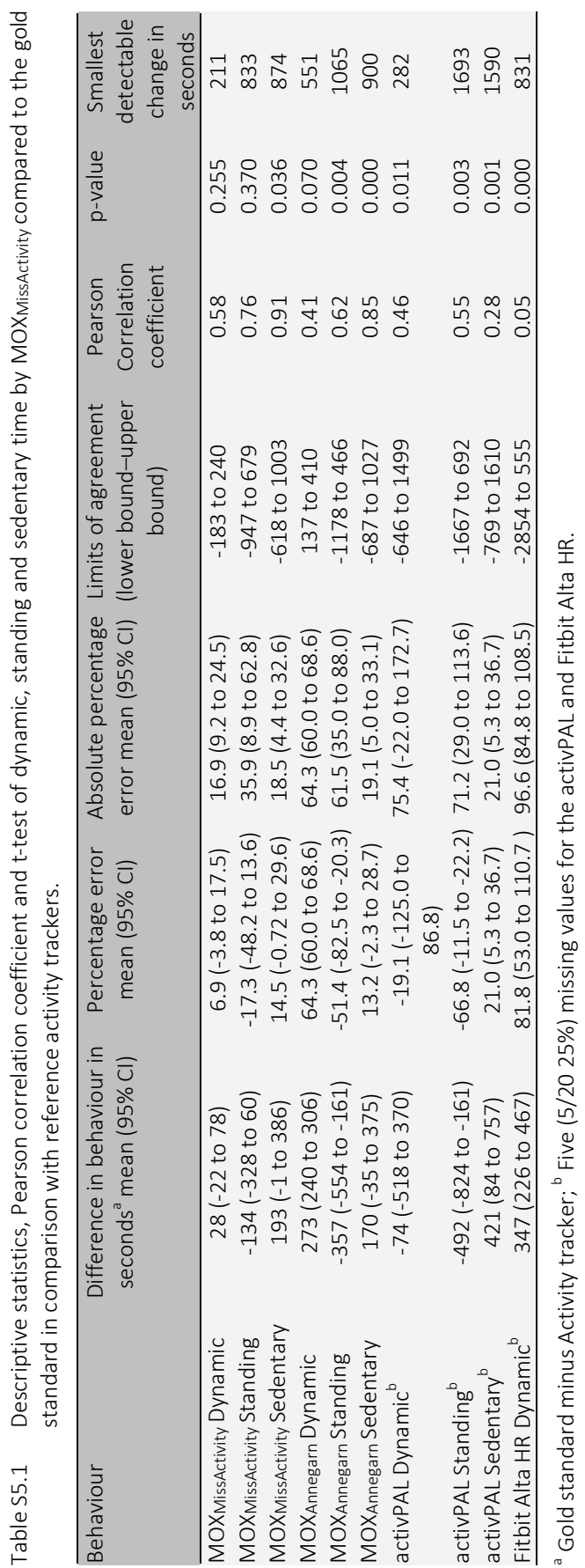


Table S5.2 Sensitivity, specificity and accuracy by MOXMissActivity compared to the gold standard in comparison with reference activity trackers without outliers.

\begin{tabular}{lccc}
\hline Behaviour & $\begin{array}{c}\text { Sensitivity without outliers, } \\
\text { mean }(95 \% \mathrm{Cl})\end{array}$ & $\begin{array}{c}\text { Specificity without outliers, } \\
\text { mean }(95 \% \mathrm{Cl})\end{array}$ & $\begin{array}{c}\text { Accuracy without outliers, } \\
\text { mean }(95 \% \mathrm{Cl})\end{array}$ \\
\hline MOXMissActivity Dynamic & $67.6(61.7-73.4)$ & $94.6(93.3-95.9)$ & $90.5(88.9-92.2)$ \\
MOX $_{\text {MissActivity Standing }}$ & $76.0(66.1-86.0)$ & $91.1(86.0-96.3)$ & $88.3(85.0-91.6)$ \\
MOX $_{\text {MissActivity Sedentary }}$ & $95.1(89.7-100.0)$ & $94.6(87.9-100.0)$ & $95.5(92.3-98.6)$ \\
MOX $_{\text {Annegarn Dynamic }}$ & $27.6(23.7-31.5)$ & $98.3(97.7-99.0)$ & $88.0(86.4-89.6)$ \\
MOX $_{\text {Annegarn Standing }}$ & $87.1(76.4-97.7)$ & $83.7(78.0-89.3)$ & $85.2(81.9-88.5)$ \\
MOX $_{\text {Annegarn Sedentary }}$ & $95.3(89.6-100.0)$ & $92.6(83.2-100.0)$ & $94.5(91.3-98.6)$ \\
activPAL Dynamic $^{a}$ & $44.3(35.6-53.0)$ & $95.0(93.8-96.2)$ & $86.9(84.6-89.4)$ \\
activPAL Standing $^{a}$ & $84.5(81.5-87.6)$ & $70.1(57.3-82.9)$ & $73.5(63.4-83.6)$ \\
activPAL Sedentary $^{a}$ & $76.5(59.4-93.5)$ & $98.4(97.5-99.4)$ & $85.0(74.4-95.6)$ \\
Fitbit Alta HR Dynamic $^{\mathrm{a}, \mathrm{b}}$ & - & - & - \\
\hline
\end{tabular}

${ }^{a}$ Five $(5 / 2025 \%)$ missing values for the activPAL and Fitbit Alta HR; ${ }^{b}$ Not possible to determine for Fitbit Alta $H R$, since Fitbit doesn't provide raw data. 


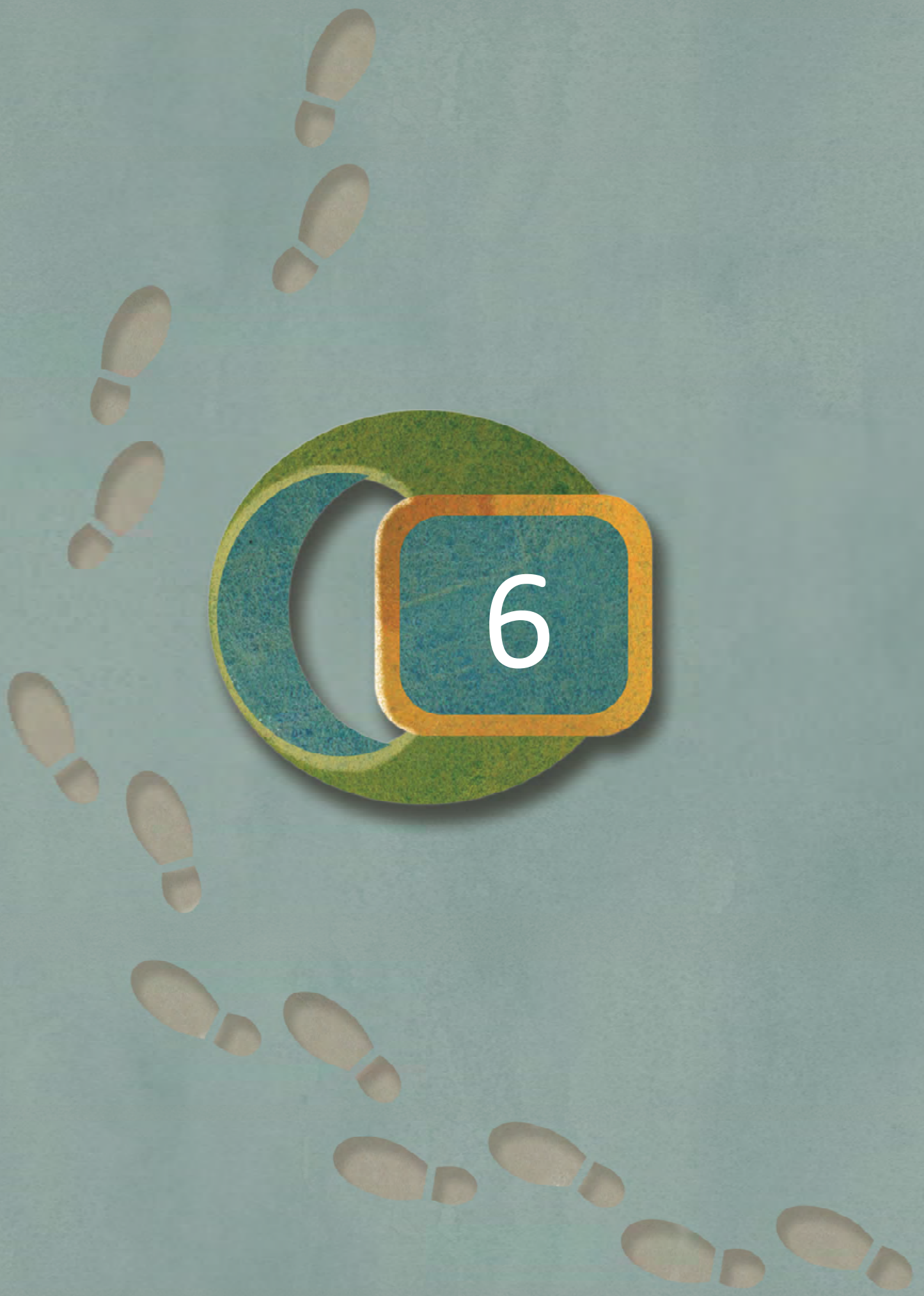




\section{Chapter 6}

Using an activity tracker in health care: experiences of healthcare professionals and patients 


\section{Abstract}

Despite the increased use of activity trackers, little is known about how they can be used in healthcare settings. This study aimed to support healthcare professionals and patients with embedding an activity tracker in the daily clinical practice of a specialized mental healthcare center and gaining knowledge about the implementation process. An action research design was used to let healthcare professionals and patients learn about how and when they can use an activity tracker. Data collection was performed in the specialized center with audio recordings of conversations during therapy, reflection sessions with the therapists, and semi-structured interviews with the patients. Analyses were performed by directed content analyses. Twenty-eight conversations during therapy, four reflection sessions, and eleven interviews were recorded. Both healthcare professionals and patients were positive about the use of activity trackers and experienced it as an added value. Therapists formulated exclusion criteria for patients, a flowchart on when to use the activity tracker, defined goals, and guidance on how to discuss (the data of) the activity tracker. The action research approach was helpful to allow therapists to learn and reflect with each other and embed the activity trackers into their clinical practice at a specialized mental healthcare center. 


\section{Introduction}

In the Netherlands in 2019, only $48 \%$ of the people with a chronic disease adhered to the physical activity recommendation. ${ }^{1}$ Sufficient physical activity has several well-known positive effects, such as prevention of premature mortality and primary and secondary prevention of chronic diseases. ${ }^{2}$ A certain amount of physical activity is also needed to participate in daily life activities, such as household, work, and social activities. ${ }^{3}$ Insight into a patient's physical activity level is an important aspect of daily practice for healthcare professionals since it is used for diagnostic, prognostic, and evaluative purposes. ${ }^{4}$ Outcomes of these measurements are a direct aid to the clinical reasoning of professionals and increase the engagement of patients in treatment. ${ }^{5}$

Questionnaires are frequently used to measure physical activity levels. A review about the measurement properties of 76 physical activity questionnaires stated that only a few had sufficient validity and reliability and none of the questionnaires could be recommended above others. ${ }^{6}$ Another review found that the reliability was acceptable for questionnaires (correlation ranging from $0.64-0.79$ ) but the validity of questionnaires was moderate at best (correlation coefficient ranging from 0.25 to 0.41 ) mainly due to patients' memory and the potential to induce social-desirability bias. ${ }^{7,8}$ A study showed that individuals experience difficulties estimating their physical activity; about half of the inactive participants in the study of Godino et al. overestimated their physical activity level and believed they were sufficiently active. ${ }^{9}$ Next to low-to-moderate clinimetric properties, questionnaires and diaries also have low feasibility because they are timeconsuming to use for both patients and healthcare professionals. ${ }^{7,8}$

Measuring physical activity with an activity tracker has advantages over the use of questionnaires or diaries. They can, for instance, objectively and continuously measure physical activity levels during daily life. Therefore, they provide insight and feedback into real-life physical activity levels, which can guide both patients and healthcare professionals in establishing and changing routine activity behavior. In addition, several systematic reviews have shown that activity trackers are effective to increase physical activity levels ${ }^{10-14}$ if they are used in combination with an intervention or counseling. Numerous studies have been performed on the measurement qualities of activity trackers, such as validity and reliability, ${ }^{15-20}$ feasibility, ${ }^{21-28}$ and effectiveness. ${ }^{10-14,22} \mathrm{~A}$ systematic review showed that there is attention to the possible added value of activity trackers in healthcare. ${ }^{29}$ These studies researched whether activity trackers were feasible in healthcare ${ }^{30}$ or can predict certain events (e.g., hospitalization, length of stay in hospital). ${ }^{31-33}$ However, in these studies, the activity trackers were only used as an outcome measure and not used in daily routine care. The research teams performed the measurements, data analyses, and conclusions, and the results were not used in 
therapy. Despite the attention to and the benefits of activity trackers, to our knowledge, no studies have been performed in which activity trackers were implemented in health care. The bottleneck of using eHealth in healthcare is often the transition from pilot phase to implementation. ${ }^{34,35}$ Several barriers and facilitators regarding the implementation of eHealth and activity tracker measurement tools in daily clinical practice are known from the literature, such as complexity of the tool, privacy/security, compatibility with existing systems, and digital health literacy. ${ }^{36-39}$

An action research design could help to transfer eHealth from the pilot phase to implementation. An action research design is not only used to develop knowledge and to understand the context but also to purposefully change this context and provide empowerment for the participants. ${ }^{40-43}$ By active participation, the participants can use the activity tracker and experience the use in daily practice. In this way, the participants gain knowledge about how and when they can use an activity tracker. This gained knowledge about activity trackers is context-specific and can directly be applied to their daily work.

The main aims of this study were to support healthcare professionals and patients with embedding an activity tracker in daily clinical practice to aid clinical reasoning and facilitate engagement of the patients in their treatment and to gain knowledge about the implementation process in clinical practice. Therefore, the following research questions were formulated: (1) How do healthcare professionals and patients use an activity tracker used in clinical practice? and (2) What are the experiences of healthcare professionals and patients with an activity tracker in clinical practice?

\section{Materials and methods}

By using an action research design, healthcare professionals and patients were given the opportunity to experience, reflect, and learn about how and when they can use an activity tracker. ${ }^{40}$ This design allows for collecting more genuine and in-depth knowledge about the participants' use and experiences. An action research design consists of four phases (Figure 6.1): (1) a plan, (2) an act and observe, (3) a reflect, and (4) a revised plan phase. 


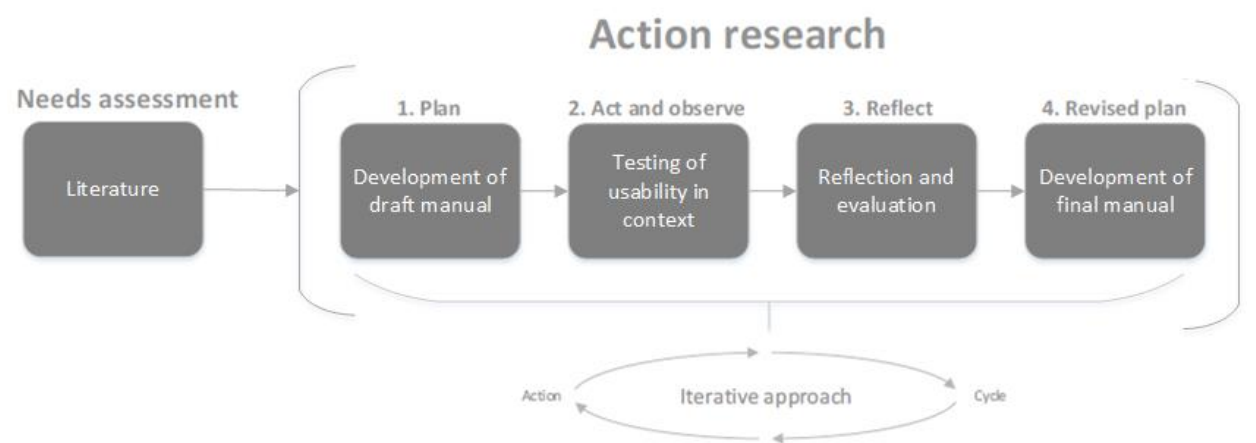

Figure 6.1 Guideline of the development process

To support the embedded use of an activity tracker in daily clinical practice, in the plan phase (1), a draft manual including a flowchart (Figure 6.2) was developed by the research team ( $D U, E B, S B, A B)$. The research team consisted of three professors and one Ph.D. student. All team members have backgrounds as physical therapists and one is still practicing as a physical therapist. The content of the manual and the flowchart was based on an earlier developed framework about the feasibility of activity trackers in healthcare and on literature about the needs of healthcare professionals and patients in using measurement tools and eHealth during treatment. ${ }^{28,36-39,45-51}$ Topics such as characteristics, correct functioning, goal, and use of the activity tracker were derived from the framework and supplemented with relevant topics (e.g., what, how, when) from literature. Following, in the act and observe phase (2), the activity tracker was used in daily clinical practice by the healthcare professionals. The act and observe phase (2) lasted for at least 4 weeks, in which part of the data collection took place. In the reflect phase (3), the rest of the data collection took place, and the draft manual was further developed by the healthcare professionals to their context and the needs of the therapists based on the gathered experiences of themselves and their patients. The act and observe phase (2), reflect phase (3), and revised plan phase (4) were iterative cycles and were planned to be repeated until there was no need for further development of the manual, i.e., when the therapists were able to embed the activity tracker in their daily clinical practice. This study was approved by the local ethics board Medical Ethical Committee METC Z (METCZ20190073). 


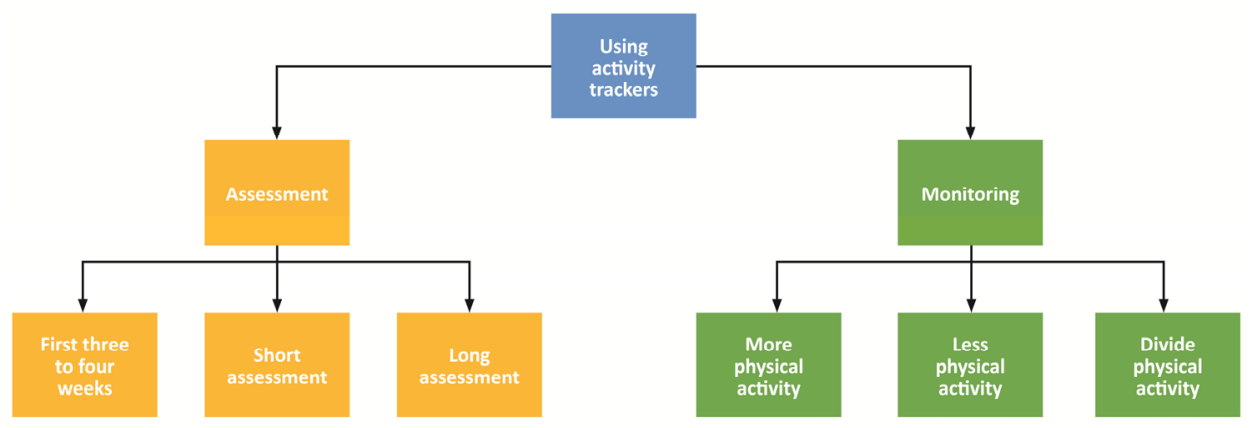

Figure 6.2 Flowchart on how to use activity trackers in daily clinical practice in the first draft manual. Blue, start using the activity tracker; yellow, assessment period; green, monitoring period. Assessment: period in which the physical activity level is assessed in the first three to four weeks for a short period ( $<2$ weeks) or a long period ( $>2$ weeks) after the initial assessment. Monitoring: period in which the patient is monitored whether they meet with their goals to be more or less physically active or to divide physical activity equally over the day.

\section{Setting and context}

The study was performed in a specialized mental healthcare center for people with chronic somatic symptom disorders specific to spinal pain (Het Rughuis Parkstad in the Netherlands). Therapy is generally provided three times a week by an interdisciplinary team over a period of six months. The team consisted of a physiotherapist, a cognitive behavioral therapist, and a psychosomatic therapist. The psychosomatic therapists were proposed to participate in this study by the specialized mental healthcare center itself since they need the most information about the physical activity level of their patients within the multidisciplinary team in order to use the pain functioning model (a theoretical framework which is used as a departure point for their approach ${ }^{52}$ ). This model describes how chronic pain can originate and can persist or worsen. Together with the patient, the consequences of their pain and influencing factors are explored. Both the bio-medical and the bio-psychological aspects are included in this model. ${ }^{52}$ This model provides insight into the pain belief, the willingness, and possibilities of the patient to reflect on their role within their pain management. With the use of this model, psychosomatic therapists divide patients into three pain-coping mechanism groups: (1) defeatism, (2) catastrophizing, and (3) non-accepting coping mechanism. The therapists use the following definitions: patients with a defeatism coping mechanism demonstrate expectation or acceptance of failure, patients with a catastrophizing coping mechanism view a situation as worse than it actually is, and patients with a non- 
accepting coping mechanism perform activities that are too burdensome for their capacity. Patients from all three groups were eligible to participate in this study.

\section{Activity tracker}

Since important barriers in implementing eHealth as a measurement tool into daily practice are the complexity, technical problems, and concerns about validity, ${ }^{36,37,39}$ it was decided to use the Measure It Super Simple activity $\operatorname{tracker}^{53}$ (in short, MISS Activity; developed by Maastricht Instruments, Maastricht, NL). The unique features of this tracker are that it measures activities of daily living more validly than other commercially available activity trackers ${ }^{19}$ and that it is user-friendly for an elderly population. ${ }^{27}$

The MISS Activity measures step count and active minutes. Active minutes are defined as time spent in dynamic behavior, with the possibility to include standing behavior as well. The data are presented as the number of steps and active minutes, including graphs showing progress to goal and the distribution of activity throughout the day (Figure 6.3). The activity tracker is clipped onto the trouser pocket.
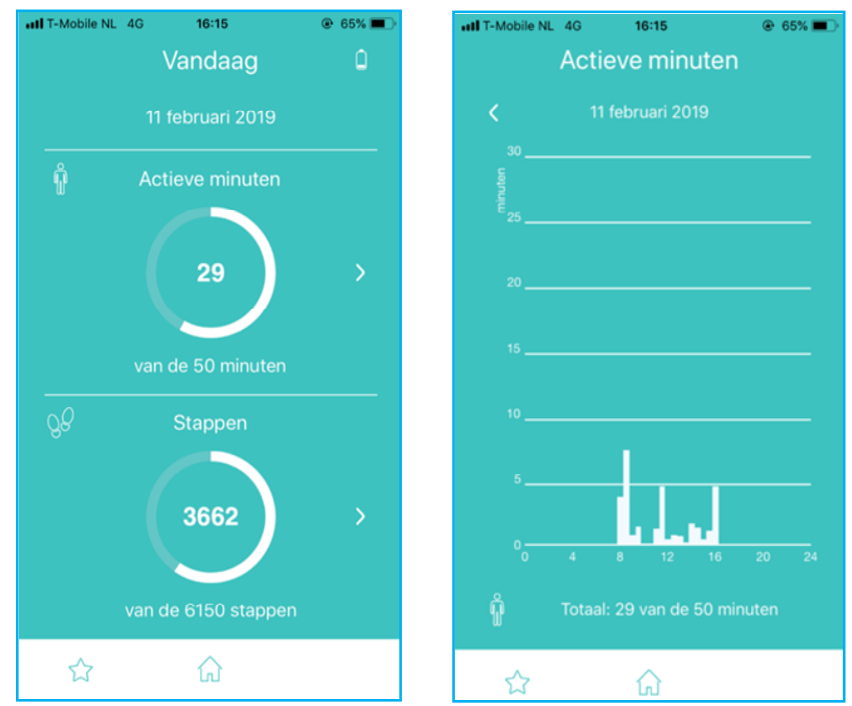

Figure 6.3 Overview of data presentation with (a) activity distribution throughout the day and (b) progress to goal. The figure shows the active minutes and number of steps per day (left) and the distribution of active minutes (or steps) over the day (right). 


\section{Participants}

Both healthcare professionals and patients were recruited in the specialized mental healthcare center. Healthcare professionals were selected via convenient sampling and were eligible if they worked as psychosomatic therapists, were motivated to use an activity tracker, and were able to participate during at least 12 months (estimated time of the entire research project). The psychosomatic therapists were recruited by the manager of the specialized mental healthcare center; no instructions were given to the manager except the inclusion criteria for the psychosomatic therapists. The psychosomatic therapists participated in all four phases of the study and recruited patients through convenient sampling. The recruited patients were individuals receiving treatment from the participating psychosomatic therapists. No instructions except from the inclusion criteria were given to the psychosomatic therapists. After giving information about the research, patients had the opportunity to consider participation for at least five working days and were included if they possessed a smartphone and provided written informed consent. Included patients participated in one cycle of the act and observe phase (2) and the reflect phase (3). New patients were included if a new act and observe phase (2) iteration was started.

\section{Data collection}

Data were collected between May 2019 and April 2020. A multi-method approach of data collection was used, consisting of audio recordings of conversations during therapy about the MISS Activity, reflection sessions with psychosomatic therapists, and semistructured individual interviews with the patients (Table 6.1). All data reflecting the use of and experiences with the MISS Activity in daily clinical practice were collected. Use is defined as how the MISS Activity is embedded in the routine of daily clinical practice (e.g., with what purpose is the activity tracker used?) and experiences are defined as how the use of the MISS Activity is experienced during daily clinical practice (e.g., do patients and therapists experience the activity tracker as meaningful?)

Table 6.1 Overview of used methods and data collection per research question.

\begin{tabular}{lcc}
\hline Data collection & Use & Experiences \\
\hline Conversations during therapy about measuring physical activity & $\mathrm{X}$ & \\
Reflection sessions with psychosomatic therapists & & $\mathrm{X}$ \\
\hline Semi-structured interview with patients & & \\
\hline
\end{tabular}




\section{Conversations during therapy about measuring physical activity}

To obtain insight into how the MISS Activity is used during therapy, each conversation in the consultation room between the patient and therapist involving the MISS Activity (15-30 min of the conversation) was recorded with an audio recorder.

\section{Reflection sessions with psychosomatic therapists}

After every act and observe phase (2), a reflection session was held with the participating therapists. The goal of these reflection sessions was to share and elaborate on how the MISS Activity was used and to share experiences regarding use in daily clinical practice. The research team (DU, AB) supported these reflection sessions by leading the session and ensuring that the draft manual and the process of clinical reasoning were discussed. As a supplementary support tool, the patient journey method $^{54}$ was used to create insight into how and when the therapists could use the MISS Activity. The patient journey method is a method to visualize the points over time when both the therapists and the patients come in contact with the MISS Activity. Participants drew a timeline and described when and how they came in contact with the MISS Activity.

Together with the therapist, the research team (DU, EB, SB, AB) improved the draft manual by adapting it to the specific context of the specialized mental healthcare center and the needs of the therapists based on the experiences of the therapists. These group sessions with the therapists and researchers took place at the specialized mental healthcare center, lasted 45-90 min, and were audio-recorded. Gender, age, years of work experience, and the number of years working at the specialized mental healthcare center were also noted during the first session.

\section{Semi-structured interviews with the patients}

After the act and observe phase (2), a semi-structured interview was conducted by DU with the involved patients to collect information on how they used the MISS Activity in their therapy and to share experiences. These results were used by the research team to improve the draft manual. The patients could choose a convenient location for the interview (e.g., home or specialized mental healthcare center). The topic list and interview guide for the interview were based on a previously developed framework which is based on expert meetings and literature. ${ }^{28}$ This framework was originally developed to assess the feasibility of activity trackers and was slightly adapted for this study. Additions were based on literature about the needs of healthcare professionals and patients regarding the use of measurement tools and eHealth as a measurement 
tool. ${ }^{28,36-39,45-51,55-63}$ The initial framework consisted of six categories: instruction, characteristics of the activity tracker, correct functioning, sharing data and privacy, goal, and use, with several subcategories (Appendix 6.1). These categories were also embedded in the draft manual. The interview lasted 15-30 min and was audio-recorded. Gender, age, and the number of treatment weeks were also noted.

\section{Data analyses}

For the data analyses, the audio recordings of the conversations during therapy, the reflection sessions, and the interviews were transcribed verbatim. Directed content analyses ${ }^{64}$ were used to analyze all data using NVivo (version 10). Deductive coding was based on the used framework (Appendix 6.1). When a text fragment was considered relevant for use or experiences with the MISS Activity but not matching with an existing code, inductive coding was used by using an "other" code. In this way, new categories or subcategories could potentially be identified and registered to the framework. The first interview and audio fragment and every fifth interview and audio fragment were coded by two researchers ( $D U$ and $\mathrm{LH}$ ), and an alignment session was held to fine-tune the coding process. Differences in interpretation were solved by dialogue to reach consensus; if needed, a third researcher was consulted. Descriptive statistics of the therapists and the patients were presented as medians (range). Data were organized in accordance with the analysis framework previously developed (Appendix 6.1).

\section{Results}

Three iteration cycles were performed within a total of 28 recorded conversations during therapy about measuring physical activity, 4 reflection sessions, and 11 semistructured interviews.

\section{Therapist' and patient' characteristics}

Three psychosomatic therapists participated in this research, of which one therapist (Therapist 3) only participated in the last reflection session. The three therapists were women $(29,33$, and 26 years old) and worked $4.5,1.5$, and 0.5 years, respectively, at the specialized mental healthcare center. In total, 11 patients were enrolled by the therapists for participation (Table 2). 
Table 6.2 Patients characteristics.

\begin{tabular}{lc}
\hline Characteristics & Participants $(n=11)$ \\
\hline Gender, $n$ male (\%) & $2(18 \%)$ \\
Age in years, (median, range) & $44(19-64)$ \\
Number of weeks in therapy, median (range) & $9(2-16)$ \\
\hline
\end{tabular}

Both the use of and the experiences with the MISS Activity during the iterations are described below. Two new categories were added: skills and beliefs (only regarding research question experiences) and goal of the activity tracker (both question use and experiences) and several subcategories were added to the category, use of the activity tracker. The results are described following the categories of the coding framework (Appendix 6.1): instruction, characteristics of the activity tracker, correct functioning, skills and beliefs, goal of the activity tracker, and use of the activity tracker. Since the categories goal of the activity tracker and use of the activity tracker were non-distinctive categories, they are described together. Following inductive analyses, the subcategory length of use was added. No third researcher was needed during the analyses.

In the use section, we reported the actual use of the activity tracker. During the course of this study, changes were made in how the activity tracker was used. The rationale behind these changes is described in the experiences section since these changes were based on the experiences of the therapists and the patients.

\section{Use of the MISS activity by healthcare professionals and patients}

In the first cycle, the therapist started using the activity tracker with the draft manual, including the summarizing flowchart (Figure 6.2). After three iterations, based on their use and experiences, several steps were added, and the flowchart was more structured according to their theoretical framework (the pain functioning model), clinical reasoning, and context (Figure 6.4). To achieve enough reflection and depth during the reflection sessions and to create this flowchart and final manual, therapists needed guidance from the research team. 


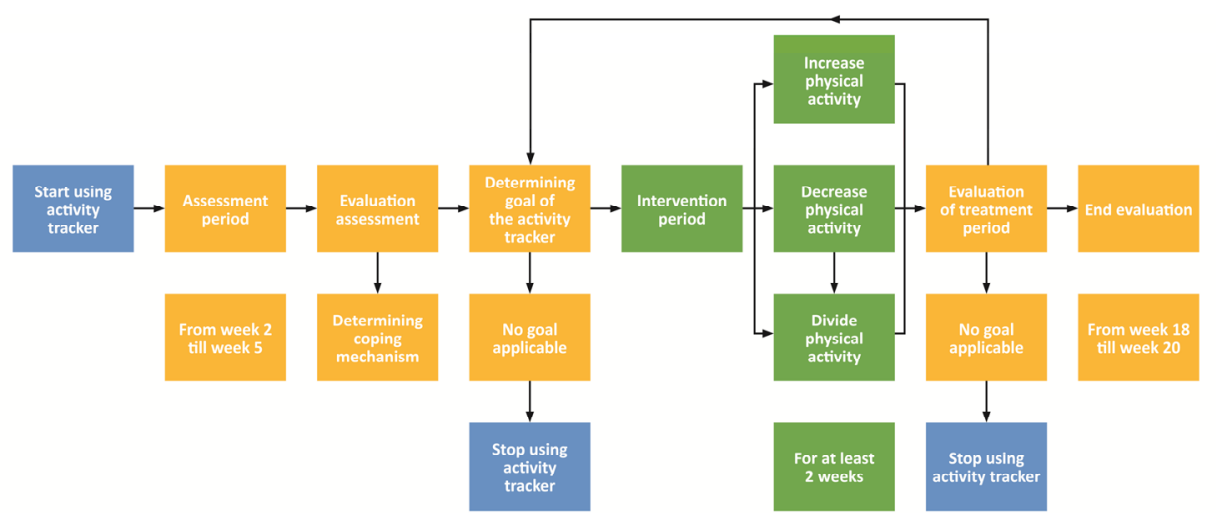

Figure 6.4 Flowchart on how to use activity trackers in daily clinical practice in the final version of the manual. Blue, starting or stopping point for using the activity tracker; yellow, assessment period; green, intervention period. Increase physical activity: to increase the daily step count or daily active minutes per day; decrease physical activity: to decrease the daily step count or daily active minutes per day; divide physical activity: to remain the same amount of steps or active minutes per day but divide the physical activity moments equally over the day.

\section{Instruction}

Therapists introduced the activity tracker to all participating patients and told them they wanted to assess their physical activity level. They explained why they thought an activity tracker could be beneficial and why they would prefer an objective measurement of the physical activity level. During the first reflection session, therapists decided to adapt the instruction; they explicitly told patients not to change their physical activity level and explained why the assessment period had value to therapists and patients as a baseline and for the intervention period. Furthermore, from the second iteration on, the therapists added more explicitly that after the assessment period, an intervention and an evaluation period would follow.

"With this activity tracker, we can objectively measure how active you are. The activity tracker will show us your actual physical activity level." -Explanation from therapist 2 to patient 3 during a therapy session (audiotaped conversation).

After the instruction, the therapists installed the activity tracker together with the patients. Therapists either performed the entire installation (e.g., downloading and synchronizing the app) or verbally explained step-by-step what patients needed to do based on the technical skills of the patient. After the application was installed, the therapist explained the user interfacee to the patients by showing them how it worked. They did not change this explanation during the iterations. 


\section{Characteristics of the activity tracker}

Throughout all iterations, therapists explained to their patients which variables the activity trackers measured, how to wear the activity tracker correctly, and the ease of use of the activity tracker.

"The only thing the activity tracker does is measure your steps and active minutes. You can charge the activity tracker at home; you just need an outlet. It is super simple." Explanation from therapist 2 to patient 11 during a therapy session (audiotaped conversation).

\section{Correct functioning}

Therapists explained to the patients that the MISS Activity is more valid and reliable than other activity trackers patients know. They did not change this explanation throughout the iterations.

"This [activity tracker] is much more reliable, it measures your steps from the couch to the kitchen, for example. Other apps and activity trackers don't measure that accurately." -Explanation from therapist 2 to patient 11 during a therapy session (audiotaped conversation).

\section{Goal of the activity tracker and use of the activity tracker}

In the first iteration, the activity tracker was only used as an assessment tool for two weeks. This was changed during the second reflection session to at least three weeks. During the first and second iteration, the standard physical activity goal of the activity tracker (5000 steps and 30 active minutes) was mostly used during the assessment period, according to the instructions in the draft manual. From the third iteration on, therapists decided to set the goal of the activity tracker during the assessment period to zero steps and zero active minutes. During the last reflection session, the therapists added that having an objective measurement of the physical activity level, along with the subjective experiences of the patient and themselves, can support them with diagnosing the coping mechanism of a patient.

"Our goal when using the MISS Activity is to gain insight into your physical activity behavior during these weeks." -Explanation from therapist 2 to patient 9 during a therapy session (audiotaped conversation).

Therapists added exclusion criteria during the second reflection session for the start of using the activity tracker because based on their clinical experiences and expertise, they considered an activity tracker not to be suitable for patients with the following characteristics: perfectionism, depression, trauma, severe physical impairment, and 
when other topics had more priority (e.g., mental health). These exclusion criteria were added to the manual.

"I have a client with heavy physiological problems and a client with traumas which I am assessing. There is no room for an activity tracker right now." -Therapist 2 (reflection session).

From the second iteration, therapists also started using the tracker as an intervention tool to support an increase or decrease in physical activity or to divide physical activity equally throughout the day. The goal to increase or decrease physical activity was chosen if the step count or active minutes per day was too high or too low in relation to the physical and mental complaints of the patient. The goal to divide physical activity equally throughout the day was chosen when the data of step count or active minutes showed several outliers in relation to the physical and mental complaints of the patient. Only three patients did not start an intervention period ( $n=1$ : due to absence of a goal related to physical activity; $n=1$ : due to the end of the study iteration cycle; $n=1$ : due to non-attendance). The physical activity goal was mostly decided by the therapist. The most frequently used physical activity goal was to divide physical activity equally throughout the day and was focused on walking a number of steps per day (function level). During the last reflection session, therapists expressed that they wanted to connect the physical activity goal of the activity tracker more explicitly to the overall participation goal of the patient. For example, to be able to walk with friends (participation goal), you have to be able to walk 6000 steps per day (physical activity goal). In the last reflection session, therapists decided that the intervention period should be at least three weeks. Moreover, therapists expressed they could also use the activity tracker during the intervention period to support treatment options such as graded activity. Therefore, both utilizations were added to the manual (intervention tool and support of an intervention).

"For a patient with a catastrophizing coping mechanism you could use graded activity or graded exposure and an activity tracker would certainly be of added value." -PS Therapist 3 (reflection session).

During the first iteration, the data of the tracker were seldom discussed by the therapist and patient. From the second iteration on, the data were discussed after the assessment period and once or twice per week during the intervention period. Therapists and patients talked about the number of steps and active minutes and whether the patient experienced the measurement period as a normal week. The app (data graphs over the past week) was used as a starting point for the conversation. In the minority of the patients, therapists and patients discussed how they experienced their symptoms (e.g., pain, fatigue) in relation to their physical activity. In only two cases, advice was given to the patient on how they could reach their physical activity goal. 
"If we look at your data, the step count is really high. 40,000 steps a day is quite a lot. Do you feel comfortable with that?" -Question from therapist 1 to patient 10 during a therapy session (audiotaped conversation).

\section{Experiences of professionals and patients with the MISS Activity}

\section{Instruction}

Throughout all iterations, therapists experienced that it was easy to explain the activity tracker to their patients. Patients expressed that the instruction of the therapists was clear enough and sufficient to start using the tracker. Both patients and therapists experienced sufficient time to give or receive instructions about the activity tracker and did not mind spending time on these instructions. Moreover, patients appreciated that the therapists downloaded and installed the app on their smartphones during the therapy session.

"We have a lot of sessions, so I have enough time to really explain the activity tracker. I notice that my clients are motivated and don't mind taking time for the instruction because they want to know how it works." -PS Therapist 1 (reflection session).

\section{Characteristics of the activity tracker}

Both patients and therapists expressed the ease of use of the activity tracker. They liked that the activity tracker was not complex and was comfortable to wear. The activity tracker measured sufficient variables, and the feedback on the activity tracker and app was clear for both therapists and patients.

'More than easy, you didn't have to explain much about it. You push that button and swipe and it appears. There is nothing hard about it.' Patient 5, female, 44 years (semistructured interview)

\section{Correct functioning}

Both the therapists and patients experienced the tracker as being valid and reliable and experienced no technical problems.

"The activity tracker really measures the number of steps. I counted my steps and looked on the app and it was the exact number!" -Patient 5, female, 44 years (semi-structured interview). 


\section{Skills and beliefs}

Most patients and all therapists found themselves skilled enough to use the activity tracker without any support. Already during the first reflection session, therapists indicated that they believed the activity tracker could be of added value for daily clinical practice. They thought that an activity tracker could be more useful than some questionnaires they used since the activity tracker provided them with objective data about their patients' physical activity level. However, during a later iteration session, therapists expressed that the combination of an activity tracker and questionnaire was particularly useful when diagnosing a coping mechanism of a patient.

"I had the opportunity to create insight, it is a nice measurement tool, clients like it in general, it can be motivating, and I like the app.' Therapist 2 (reflection session)

Patients found it convenient that the activity tracker measures all their activities since they mostly were not aware of every single activity they performed and therefore did not note them in their diary. Patients expressed the convenience of the visual results of the activity tracker (i.e., data graphs); without it, they would have found it difficult to explain the physical activity level to their therapists. Patients mainly valued the assessment period; they liked the activity tracker as a tool to gain insight into their physical activity, and it confronted them with their own behavior. Other patients believed that the activity tracker data made it easier to show their therapists their physical activity level. Moreover, they also thought it was fun to use the activity tracker. During the intervention period, some patients experienced a positive stimulation from the activity tracker while others thought that the data from the activity tracker resulted in negative pressure.

'I really valued that I could see how my physical activity is related to my pain and fatigue.' Patient 9, female, 25 years (semi-structured interview)

'I have to remember keeping my diary and, apparently, I am more active than I thought based on the activity tracker. I think I wouldn't write all the activities in my diary. For example, when I run out of toilet paper, I walk to my basement to get some new rolls. I wouldn't write that down as an activity.' Patient 1, female, 35 years (semi-structured interview)

\section{Goal of the activity tracker and use of the activity tracker}

Therapists were positive about the objective insight they got from an activity tracker during the assessment and intervention period. During the assessment period, they noticed that, in general, there was a mismatch between the experienced physical activity level and the actual physical activity level of their patient. During the last reflection session, they discussed how the use of an activity tracker can support them with 
diagnosing the coping mechanism of a patient and opt for treatment strategies such as graded activity. The diagnosis of the coping mechanism can be supported by the activity tracker, since part of the diagnosis is the agreement between the objective physical activity level (i.e., how physically active somebody actually is) and the subjective physical activity level (i.e., how physically active somebody thinks he/she is). They also noticed that the objective measurement provided insight for patients into their own coping mechanisms.

'It is important to objectively know how physically active they [patients] are. They tell you they are very active but, if they aren't active, that is non-accepting pain-coping. Therapist 3 (reflection session)

Therapists also observed that some patients did not want to talk about their physical activity level. Therapists suspected that this was because the objective measurement revealed the actual problem for the patient (i.e., coping mechanism). During the last reflection session, therapists indicated that they would like to guide the conversation more towards the activity tracker data even when patients do not want to talk about it.

'They don't want to talk about the activity tracker, because it is the core of their problem; they keep being too active and keep being chaotic. It really can be good to reflect on that' Therapist 1 (reflection session)

During the first and second reflection sessions, therapists expressed their difficulties in deciding on an appropriate physical activity goal (i.e., number of steps or active minutes) for their patients during the assessment period. During the second reflection session, therapists decided that the standard goal during the assessment period should be zero steps and zero active minutes for everybody so that patients would not feel the pressure of the standard physical activity goals during the assessment period.

'You never know how physically active somebody is, so you always have to guess a goal. For example, with patient two, I thought he wasn't active so I set his goal in the assessment period at 1,000 steps but he walked 9,000 steps.' PS Therapist 1 (reflection session)

Both therapists and patients explained that there was sufficient time to discuss the data of the activity tracker. Patients valued these conversations but would like more guidance on how to reach their physical activity goals. Patients indicated that it was important that the time interval between measuring their physical activity or goal setting and discussing the data was not too long ( $>1$ week) otherwise, they started to self-interpret the data. They felt the need for reassurance that their goal was sufficient.

During the last reflection session, therapists noted that patients did try to achieve the physical activity goals (number of steps) during the intervention period but often did not manage to do so and often changed their goals independently. Patients indicated that due to the experienced lack of guidance by their therapists during the intervention 
period, they set their own physical activity goals, often to 10,000 steps per day. They argued that this goal is often communicated in society as a healthy number of steps per day, but it was hard to reach and when they did not use the activity trackers, they relapsed into their old behavior. Reasons mentioned by the therapists why patients did not manage their physical activity goals or altered their goals were, among others, that patients were not ready for a behavioral change or the intervention period was too short. Therefore, they decided to expand the intervention period to a minimum of three weeks instead of the suggested one or two weeks in the draft manual and to tailor the physical activity goal of the activity tracker more to goals on participation level (e.g., increase step count to be able to walk with friends). This was altered in both the manual and flowchart.

'We lowered the goal but in some way it didn't feel right. I just couldn't do it, I couldn't manage to take some rest, being active is part of my lifestyle.' Patient 11, female, 35 years (semi-structured interview)

'People were very goal-oriented and kept walking to reach their goal, but they lost motivation because they got bored, but if they do something they liked it to easily reach 4,000 or 5,000 steps.' PS Therapist 2 (reflection session)

\section{Discussion}

This study aimed to support healthcare professionals and patients with embedding an activity tracker in the daily clinical practice of a specialized mental healthcare center. It also aimed to gain knowledge about the implementation process of an activity tracker in clinical practice. In order to do so, an action research design was used.

Both healthcare professionals and patients were positive about the use of activity trackers and experienced it as an added value in therapy. The action research approach with multiple iterations supported the learning and reflection process of the therapists on their own behavior and in learning from and with each other. In this way, they were able to discover the opportunities of the activity tracker within their context. In actuality, the support of the researchers during the reflection sessions was needed to achieve sufficient depth. The therapists were able to embed the MISS Activity in daily clinical practice using the pain functioning model as a theoretical framework. They formulated specific exclusion criteria for patients, adapted the flowchart on when to use the activity tracker and with which assessment and intervention goals, used the activity tracker to support identifying coping mechanism, and formulated guidance on how to discuss (the data of) the activity tracker. During the third reflection session, new insights were 
discussed. Unfortunately, due to the closing of the specialized mental healthcare center during the COVID-19 pandemic, it was not possible to add a fourth iteration.

\section{Comparison to other studies}

Our findings are comparable with another participatory action study that focused on the implementation of eHealth in specialist nursing teams who case-managed patients with chronic obstructive pulmonary disease and chronic heart failure and who were using telehealth to monitor patients' vital signs and symptoms. ${ }^{65}$ They formulated seven main areas of work in their implementation plan: (1) establishing a telehealth pathway, (2) improving patient assessment and review, (3) improving service delivery, (4) improving data sharing and access, (5) raising awareness of telehealth, (6) improving the evaluation of telehealth, and (7) securing financial investment for telehealth. Some areas are comparable to this study, and other areas were not within the scope of this study, such as securing financial investments. However, these topics are also important and could be further assessed in further research. An important difference between the study of Taylor et al. and our study is that healthcare professionals already had experience with the use of telehealth in their daily clinical practice. ${ }^{66}$ Other studies showed that if healthcare professionals are already experienced in using eHealth, they report fewer implementation barriers and experience more advantages (e.g., more positive attitude towards eHealth). ${ }^{66,67}$

Many of the facilitators and barriers for implementation are equal for eHealth measurement tools and other measurement tools, such as questionnaires. ${ }^{34,36-39,68}$ The review of Foster et al. emphasized the importance of involving the target population and allowing them to learn and reflect on the use of the measurement tool and guide them through the whole implementation process. ${ }^{68}$ In our study, we started with a draft manual, based on literature, on how to use activity trackers/eHealth in daily clinical practice, which was redeveloped by experience-based testing by the therapists, and guidance by the research team was given during the reflection sessions. The design and approach of this study could be used as an example for other implementation studies. The topics of security and compatibility with existing systems were not within the scope of this study, and the topics within our coding framework sharing data and privacy (e.g., safely sharing data and warrant of privacy) were not discussed by the therapists in this study but are also relevant factors for implementation. ${ }^{34,36-39}$

\section{Methodological quality}

This study had some limitations. First, there was a limited number of therapists and patients included, and the therapists had relatively short work experience. More 
experienced therapists might have integrated the activity trackers faster or differently. On the other hand, it might be possible that younger therapists are more open to working with eHealth. Second, by using convenience sampling, there might have occurred selection bias for the therapists. As mentioned above, the selected therapists might be already more open to working with eHealth in comparison to their colleagues. This is an advantage for participation in action research because active participation is required. In future studies, other therapists should be involved in using the developed manual. Convenience sampling was also applied for the recruitment of the patients; however, this could also be beneficial for the action research design since therapists were free in choosing the patients, based on their clinical expertise, who might benefit from the use of the activity tracker, a situation that is closely related to the situation in daily healthcare. Thereby, they had the opportunity to formulate exclusion criteria for patients based on their experiences during this study. But we cannot rule out selection bias. Third, due to the COVID-19 outbreak, the study had to be ended after the third iteration. In the third iteration, therapists expressed additional new methods to support their clinical reasoning with the use of the activity tracker. One more iteration would have allowed for the evaluation of these planned changes in their clinical reasoning and to facilitate the engagement of patients. Fourth, the therapists experienced that patients did not always show up at the therapy meetings, which potentially affected their own and patients' experiences with the activity tracker. It is known that missing therapy meetings happens regularly in long-term treatments, ${ }^{69}$ and thus the use of an activity tracker was not likely to be the reason for the current compliance of the patients in this study.

A strength of this study was the use of a draft manual based on earlier research and the use of the coding framework (see Appendix 6.1). The draft manual gave guidance during the implementation process and could be tailored during the reflection sessions to the specific context. The framework was based on an earlier framework developed to gain insight into the important concepts of experiences with an activity tracker. ${ }^{28}$ However, not all (sub)categories were used during this study because some did not fit within the scope of this study. Another strength of this study is the use of the MISS Activity that anticipated formerly mentioned important implementation barriers, such as complexity, technical problems, and concerns about validity. By eliminating those barriers, this study allows for a more in-depth study of the use of the activity tracker in daily clinical practice, and more genuine experiences could be collected.

To ensure the quality and trustworthiness of this study, credibility and transferability were checked in several ways. ${ }^{70}$ Method, investigator, and data triangulation were used to ensure credibility. Multiple methods of data collection were used (audio recordings of conversations, reflection sessions, and interviews); all authors reflected on the design, 
data collection, and analyses to ensure investigator triangulation; and different sources of the same information were used (multiple interviewees) to achieve data triangulation. By providing a thick description of our study population and study process, transferability was assured.

\section{Clinical relevance}

This study was performed in a specialized mental healthcare center for people with chronic somatic symptom disorders specific to spinal pain. However, even though this study was performed in this specific setting, the approach and results are still generalizable to a broader context where measuring physical activity is important. Measuring is an important aspect of almost all healthcare professionals' daily routines. The availability of eHealth tools, including activity trackers, is growing and its relevance has already been shown during the COVID-19 pandemic. Moreover, the relevance of using activity trackers is already recommended in guidelines for healthcare professionals such as physical therapists. ${ }^{71}$ In order to use eHealth and thus activity trackers in a meaningful way, healthcare professionals require new competencies, so-called eHealth competencies. ${ }^{72,73}$ Current healthcare professionals have not been sufficiently trained in these new competencies for optimal use in daily healthcare. It is important not only to focus on eHealth devices but, in addition, on how to embed them in processes of clinical reasoning and discussions with the patient and to support and train healthcare professionals to gain these competencies. An action research design could be beneficial to achieve this transition. Further research should focus on optimally embedding the activity tracker in healthcare, and our approach could be an example of how to implement eHealth in combination with healthcare professionals in their daily clinical practice. The draft manual and framework can be used completely or partially in other studies to assess the feasibility and facilitate the use of activity trackers in daily clinical practice. Consequently, a next step can be to evaluate the effectiveness of embedded activity trackers in daily clinical practice.

\section{Conclusion}

Therapists did identify opportunities to embed the use of the activity tracker into their clinical reasoning and engage patients in their treatment. Based on their expertise and experiences, therapists had clear ideas about for whom the use of activity trackers could be beneficial. They were able to formulate specific exclusion criteria accordingly (e.g., depression). An important part of this study was the adaptation of the flowchart. Each 
iteration was a source for improvement, and several times, fine-tuning of the flowchart took place. The flowchart included when to use the activity tracker and with which goals, which could either be assessment goals or intervention goals (more physical activity, less physical activity, or dividing physical activity over the day). Furthermore, therapists formulated how they could use the activity tracker as a support tool to identify the coping mechanism of a patient. Finally, the therapists were able to tailor the manual.

The action research approach with multi-iterations was needed to support professionals and embed the activity tracker in their daily clinical practice within a specialized mental healthcare center. For future studies and implementation processes, it is important to remember that healthcare professionals need time to learn how to use such innovation and reflect on this use in daily clinical practice. It is important that healthcare professionals can learn from and with each other and receive sufficient support and guidance during the implementation process and feedback from patients. The design of this study can be used as an example when implementing innovations in healthcare settings and parts of the results can be transferred to other healthcare settings (e.g., primary care settings).

\section{Acknowledgments}

We would like to thank all therapists and clients from Het Rughuis Parkstad who participated in this research. Furthermore, we would like to thank Laura Hochstenbach for her help during the analyses of the data. 


\section{References}

1. Het aandeel van de Nederlandse Bevolking van 4 Jaar en Ouder dat Voldoet aan de Beweegrichtlijnen. Available online: https://www.sportenbewegenincijfers.nl/kernindicatoren/beweegrichtlijnen (accessed on 2 October 2020).

2. Warburton DE, Bredin SS. Health benefits of physical activity: A systematic review of current systematic reviews. Curr Opin Cardiol. 2017;32:541-56.

3. Verbrugge LM, Jette AM. The disablement process. Soc Sci Med. 1994;38:1-14.

4. Raamwerk Klinimetrie Voor Evidence Based Products. Available online: https://www.kngf.nl/ binaries/content/assets/kngf/onbeveiligd/vak-en-kwaliteit/kwaliteit/klinimetrie/toepassing-raamwerkupdate-november-2016.pdf (accessed on 14 January 2020).

5. Beurskens A, Swinkels R, Wittink H, van Peppen R, Vermeulen H. Meten in de Praktijk, 1st ed.; Bohn Stafleu van Loghum: Houten, The Netherlands, 2020.

6. Van Poppel MNM, Chinapaw MJM, Mokkink LB, Van Mechelen W, Terwee CB. Physical activity questionnaires for adults: A systematic review of measurement properties. Sports Med. 2010;40: 565-600.

7. Helmerhorst HJF, Brage S, Warren J, Besson H, Ekelund U. A systematic review of reliability and objective criterion-related validity of physical activity questionnaires. Int J Behav Nutr Phys Act. 2012;9:103.

8. Ainsworth B, Cahalin L, Buman M, Ross R. The Current State of Physical Activity Assessment Tools. Prog Cardiovasc Dis. 2015;57:387-95.

9. Godino JG, Watkinson C, Corder K, Sutton S, Griffin SJ, Van Sluijs EMF. Awareness of physical activity in healthy middle-aged adults: A cross-sectional study of associations with sociodemographic, biological, behavioural, and psychological factors. BMC Public Health 2014;14:421.

10. Oliveira JS, Sherrington C, Zheng ERY, Franco MR, Tiedemann A. Effect of interventions using physical activity trackers on physical activity in people aged 60 years and over: A systematic review and metaanalysis. Br J Sports Med. 2020;54:1188-94.

11. Brickwood KJ, Watson G, O'Brien J, Williams AD. Consumer-Based Wearable Activity Trackers Increase Physical Activity Participation: Systematic Review and Meta-Analysis. JMIR mHealth uHealth 2019;7: e11819.

12. Lynch C, Bird S, Lythgo N, Selva-Raj I. Changing the Physical Activity Behavior of Adults With Fitness Trackers: A Systematic Review and Meta-Analysis. Am J Health Promot. 2019;34:418-30.

13. Franssen WMA, Franssen GHLM, Spaas J, Solmi F, Eijnde BO. Can consumer wearable activity trackerbased interventions improve physical activity and cardiometabolic health in patients with chronic diseases? A systematic review and meta-analysis of randomised controlled trials. Int J Behav Nutr Phys Act. 2020;17:1-20.

14. Cheatham SW, Stull KR, Fantigrassi M, Motel I. The efficacy of wearable activity tracking technology as part of a weight loss program: A systematic review. J Sports Med Phys Fit. 2017;58:534-48.

15. Straiton N, Alharbi M, Bauman A, Neubeck L, Gullick J, Bhindi R, Gallagher R. The validity and reliability of consumer-grade activity trackers in older, community-dwelling adults: A systematic review. Maturitas 2018;112:85-93.

16. Evenson KR, Goto MM, Furberg RD. Systematic review of the validity and reliability of consumer-wearable activity trackers. Int J Behav Nutr Phys Act. 2015;12:1-22.

17. O'Driscoll R, Turicchi J, Beaulieu K, Scott S, Matu J, Deighton K, Finlayson G, Stubbs J. How well do activity monitors estimate energy expenditure? A systematic review and meta-analysis of the validity of current technologies. Br J Sports Med. 2018;54:332-40.

18. Ummels D, Beekman E, Theunissen K, Braun S, Beurskens AJ. Counting Steps in Activities of Daily Living in People With a Chronic Disease Using Nine Commercially Available Fitness Trackers: Cross-Sectional Validity Study. JMIR mHealth uHealth 2018;6:e70.

19. Ummels D, Bijnens W, Aarts J, Meijer K, Beurskens AJ, Beekman E. The Validation of a Pocket Worn Activity Tracker for Step Count and Physical Behavior in Older Adults during Simulated Activities of Daily Living. Gerontol Geriatr Med. 2020;6:2333721420951732. 
20. Hartung V, Sarshar M, Karle V, Shammas L, Rashid A, Roullier P, Eilers C, Mäurer M, Flachenecker P, Pfeifer K, et al. Validity of Consumer Activity Monitors and an Algorithm Using Smartphone Data for Measuring Steps during Different Activity Types. Int J Environ Res Public Health 2020;17:9314.

21. Carrasco JJ, Pérez-Alenda S, Casaña J, Soria-Olivas E, Bonanad S, Querol F. Physical Activity Monitoring and Acceptance of a Commercial Activity Tracker in Adult Patients with Haemophilia. Int J Environ Res Public Health 2019;16:3851.

22. Ridgers ND, McNarry MA, Mackintosh KA. Feasibility and Effectiveness of Using Wearable Activity Trackers in Youth: A Systematic Review. JMIR mHealth uHealth 2016;4:e129.

23. Maher C, Ryan J, Ambrosi C, Edney S. Users' experiences of wearable activity trackers: A cross-sectional study. BMC Public Health 2017;17:880.

24. Karapanos E, Gouveia R, Hassenzahl M, Forlizzi J. Wellbeing in the Making: Peoples' Experiences with Wearable Activity Trackers. Psychol. Well-Being 2016;6:4.

25. Mercer K, Giangregorio L, Schneider E, Chilana P, Li M, Grindrod K. Acceptance of Commercially Available Wearable Activity Trackers Among Adults Aged Over 50 and With Chronic Illness: A Mixed-Methods Evaluation. JMIR mHealth uHealth 2016;4:e7.

26. Ehn M, Eriksson LC, Åkerberg N, Johansson AC. Activity Monitors as Support for Older Persons' Physical Activity in Daily Life: Qualitative Study of the Users' Experiences. JMIR mHealth uHealth 2018;6:e34.

27. Ummels D, Braun S, Stevens A, Beekman E, Beurskens A. Measure It Super Simple (MISS) activity tracker: (re)design of a user-friendly interface and evaluation of experiences in daily life. Disabil Rehabil Assist Technol. 2020;1-11.

28. Ummels D, Beekman E, Moser A, Braun SM, Beurskens AJ. Patients' experiences with commercially available activity trackers embedded in physiotherapy treatment: A qualitative study. Disabil Rehabil 2020;42:3284-92.

29. Shin G, Jarrahi MH, Fei Y, Karami A, Gafinowitz N, Byun A, Lu X. Wearable activity trackers, accuracy, adoption, acceptance and health impact: A systematic literature review. J Biomed Inform. 2019;93: 103153.

30. Chum J, Kim MS, Zielinski L, Bhatt M, Chung D, Yeung S, Litke K, McCabe K, Whattam J, Garrick L, et al. Acceptability of the Fitbit in behavioural activation therapy for depression: A qualitative study. Évid Based Ment Health 2017;20:128-33.

31. Sprint G, Cook D, Weeks D, Dahmen J, La Fleur A. Analyzing Sensor-Based Time Series Data to Track Changes in Physical Activity during Inpatient Rehabilitation. Sensors 2017;17:2219.

32. Lin Y, Chung Y, You C, Chuang Y, Liu B, Chang H, Huang M. Neither One is Enough: Exploring the Use of Wrist-worn Activity Trackers to Assist Acute Psychiatric Healthcare. In Proceedings of the ACM Conference, Hong Kong, China, 28 June-2 July 2018.

33. Gresham G, Hendifar AE, Spiegel B, Neeman E, Tuli R, Rimel BJ, Figlin RA, Meinert CL, Piantadosi S, Shinde AM. Wearable activity monitors to assess performance status and predict clinical outcomes in advanced cancer patients. NPJ Digit Med. 2018;5:27.

34. Wouters M, Huygens M, Voogdt H, Meurs M, de Groot J, de Bruin K, Brabers A, Hofstede C, Friele R, van Gennip L. E-Health Monitor: Samen aan Zet! Nictiz and Nivel, 2019. Available online: https://www.narcis.nl/publication/RecordID/publicat\%3A1003604 (accessed on 22 October 2019).

35. Actieplan Ehealth Implementatieonderzoek. Available online: https://www.zonmw.nl/fileadmin/ zonmw/documenten/LSH/Actieplan_eHealth/Actieplan_ehealth_implementatieonderzoek_april_2013.p df (accessed on 6 February 2021).

36. Schreiweis B, Pobiruchin M, Strotbaum V, Suleder J, Wiesner M, Bergh B. Barriers and Facilitators to the Implementation of eHealth Services: Systematic Literature Analysis. J Med Internet Res. 2019;21:e14197.

37. Ross J, Stevenson F, Lau R, Murray E. Factors that influence the implementation of e-health: A systematic review of systematic reviews (an update). Implement. Sci. 2016;11: 1-12.

38. Kruse CS, Karem P, Shifflett K, Vegi L, Ravi K, Brooks M. Evaluating barriers to adopting telemedicine worldwide: A systematic review. J Telemed Telecare 2018;24:4-12.

39. Granja C, Janssen W, Johansen MA. Factors Determining the Success and Failure of eHealth Interventions: Systematic Review of the Literature. J Med Internet Res. 2018;20:e10235.

40. Koshy E, Koshy V, Waterman H. Action Research in Healthcare, 1st ed.; SAGE: London, UK, 2011.

41. Karim K. Assessing the strengths and weaknesses of action research. Nurs Stand. 2001;15:33-5. 
42. Migchelbrink F. De kern van Participatief Actieonderzoek, 1st ed.; Uitgeverij SWP: Amsterdam, The Netherlands, 2016.

43. Casey M, Leary DO, Coghlan D. Unpacking action research and implementation science: Implications for nursing. J Adv Nurs. 2017;74:1051-8.

44. Van Dongen JJJ, Van Bokhoven MA, Goossens WNM, Daniëls R, Van Der Weijden T, Beurskens A. Development of a Customizable Programme for Improving Interprofessional Team Meetings: An Action Research Approach. Int J Integr Care 2018;18:8.

45. De Vries H, Kloek C, Bossen D, Veenhof C. Fysiotherapeutisch gebruik van een blended e-healthinterventie: Waarom wordt e-health (niet) gebruikt? Fysiopraxis 2016;3:35-7.

46. Andrieu N, Mannens L, Nowak K. Toepasbaarheid en Hanteerbaarheid van Activiteitenmeters Binnen de Ergotherapie bij COPD-Patiënten. Bachelor's Thesis, Zuyd University of Applied Science, Heerlen, The Netherlands, 2015.

47. Van Bommel M, Van Gisbergen T. De Eerste Stap zetten. De Hanteerbaarheid en Toepasbaarheid van Activiteitenmeters. Bachelor's Thesis, Zuyd University of Applied Science, Heerlen, The Netherlands, 2015.

48. Schins J, Fisscher J. Het Gebruik van de MISS Activity Door de Ogen van Patiënten Met COPD. Bachelor's Thesis, Zuyd University of Applied Sciences, Heerlen, The Netherlands, 2020.

49. Kusters A, Borrmann A. Meningen van Patiënten met Claudicatio Intermittens over het Gebruik van de Activiteitenmeter MISS Activity in de Fysiotherapie. Bachelor's Thesis, Zuyd University of Applied Sciences, Heerlen, The Netherlands, 2020.

50. Mancuso PJ, Thompson M, Tietze M, Kelk S, Roux G. Can Patient Use of Daily Activity Monitors Change Nurse Practitioner Practice? J Nurse Pract. 2014;10:787-93.e4.

51. Verwey R, van der Weegen $\mathrm{S}$, Tange,H, Spreeuwenberg M, van der Weijden $\mathrm{T}$, de Witte L. Get moving: The practice nurse is watching you! A case study of the user-centred design process and testing of a webbased coaching system to stimulate the physical activity of chronically ill patients in primary care. Inform Prim Care 2013;20:289-98.

52. Gijsen A, Huijnen I, Palmen A, Köke A, Geilen M. Toepassing in de fysiotherapiepraktijk Het pijngevolgenmodel. Fysiopraxis 2018;12:18-20

53. MISS Activity. Available online: https://www.accelerometry.eu/miss-activity/ (accessed on 22 October 2019).

54. Stinkdorn M. This Is Service Design Doing, 1st ed.; O'Reilly Media: Sebastopol, CA, USA, 2018.

55. Armağan K, Çiğdem E. Design for physical activity: Design aspects of wearable activity trackers. In Proceedings of the International Conference of Engineering Design, Milano, Italy, 27-30 June 2015.

56. Lazar A, Koehler C, Tanenbaum J, Nguyen DH. Why We Use and Abandon Smart Devices; Ubicomp: Osaka, Japan, 2015.

57. Rosenberg D, Kadokura EA, Bouldin ED, Miyawaki CE, Higano CS, Hartzler AL. Acceptability of Fitbit for physical activity tracking within clinical care among men with prostate cancer. In Proceedings of the AMIA Annual Symposium Proceedings, Washington, DC, USA, 4-8 November 2016.

58. Fausset CB, Mitzner TL, Price CE, Jones BD, Fain BW, Rogers WA. Older Adults' Use of and Attitudes toward Activity Monitoring Technologies. Proc Hum Factors Ergon Soc. 2013;57:1683-7.

59. O'Brien T, Troutman-Jordan M, Hathaway D, Armstrong S, Moore M. Acceptability of wristband activity trackers among community dwelling older adults. Geriatr Nurs. 2015;36 (Suppl. 2):S21-5,

60. Harrison D, Marshall P, Bianchi-Berthouze N, Bird J. Activity Tracking; Barriers, Workarounds and Customisation; UbiComp.: Osaka, Japan, 2015.

61. Shih PC, Han K, Poole ES, Rosson MB, Carroll JM. Use and Adoption Challenges of Wearable Activity Trackers. In Proceedings of the iConference Proceedings, Newport Beach, CA, USA, 24-27 March 2015.

62. Stevens A, Beurskens A, Köke A, Van Der Weijden T. The use of patient-specific measurement instruments in the process of goal-setting: A systematic review of available instruments and their feasibility. Clin Rehabil. 2013;27:1005-19.

63. Lyons EJ, Lewis ZH, Mayrsohn BG, Rowland JL. Behavior Change Techniques Implemented in Electronic Lifestyle Activity Monitors: A Systematic Content Analysis. J Med Internet Res. 2014;16:e192.

64. Hsieh HF, Shannon SE. Three Approaches to Qualitative Content Analysis. Qual Health Res. 2005;15: 1277-88. 
65. Taylor J, Coates E, Wessels B, Mountain G, Hawley MS. Implementing solutions to improve and expand telehealth adoption: Participatory action research in four community healthcare settings. BMC Health Serv Res. 2015;15:529.

66. Ariens LF, Schussler-Raymakers FM, Frima C, Flinterman A, Hamminga E, Arents BW, Bruijnzeel-Koomen CA, De Bruin-Weller MS, Van Os-Medendorp H. Barriers and Facilitators to eHealth Use in Daily Practice: Perspectives of Patients and Professionals in Dermatology. J Med Internet Res. 2017;19:e300.

67. Ålander T, Scandurra I. Experiences of Healthcare Professionals to the Introduction in Sweden of a Public eHealth Service: Patients' Online Access to their Electronic Health Records. Stud Health Technol Inform. 2015;216:153-7.

68. Foster A, Croot L, Brazier J, Harris J, O'Cathain A. The facilitators and barriers to implementing patient reported outcome measures in organisations delivering health related services: A systematic review of reviews. J Patient-Rep Outcomes 2018;2:1-16.

69. De Geest S, Sabaté E. Adherence to Long-Term Therapies: Evidence for Action. Eur J Cardiovasc Nurs. 2003;2:323.

70. Lincoln YS, Guba EG. Naturalistic Inquiry; SAGE Publications Ltd.: London, UK, 1985; ISBN 0803924313.

71. Vreeken HL, Beekman E, Van Doornmaal M, Post MHL, Meerhoff GA, Spruit MA. KNGF-Richtlijn COPD: KNGF; 2020. Available online: https://www.kngf2.nl/kennisplatform/richtlijnen/copd-2020 (accessed on 28 August 2021)

72. Barakat A, Woolrych RD, Sixsmith A, Kearns WD, Kort HS. eHealth Technology Competencies for Health Professionals Working in Home Care to Support Older Adults to Age in Place: Outcomes of a Two-Day Collaborative Workshop. Medicine 2.0 2013;2:e10.

73. Brunner M, McGregor D, Keep M, Janssen A, Spallek H, Quinn D, Jones A, Tseris E, Yeung W, Togher L, et al. An eHealth Capabilities Framework for Graduates and Health Professionals: Mixed-Methods Study. J Med Internet Res. 2018;20:e10229. 


\section{Appendix 6.1}

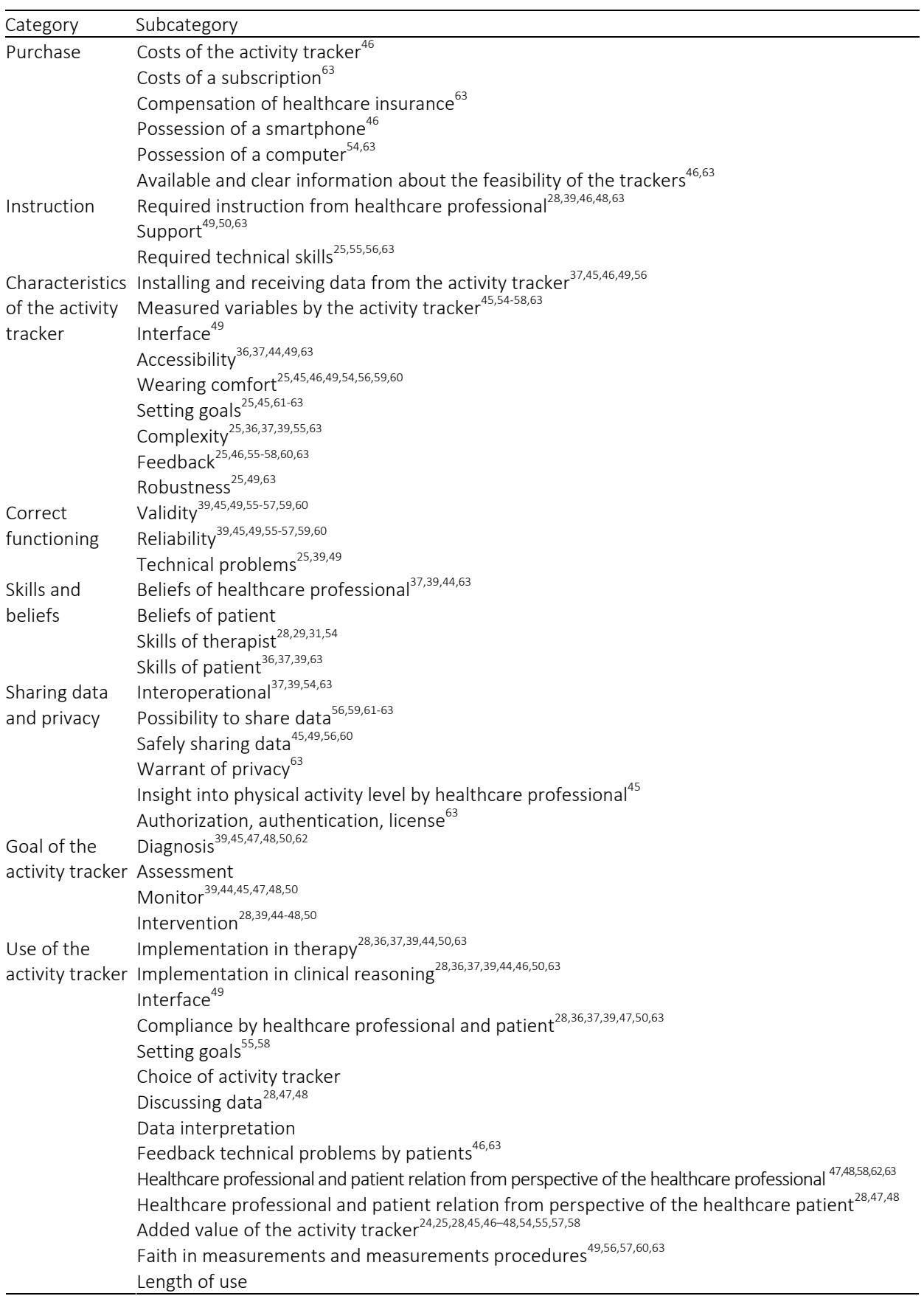




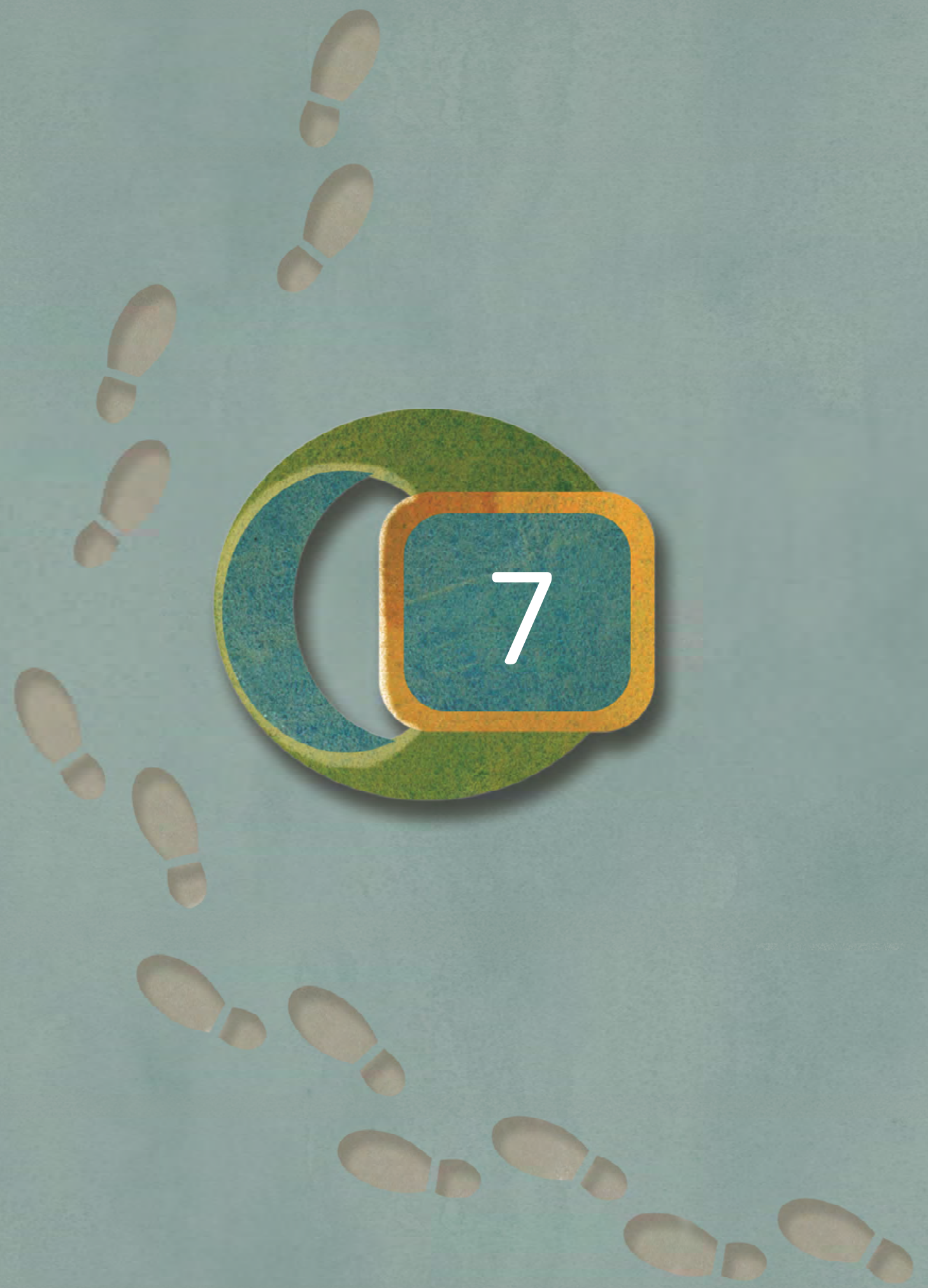


Chapter

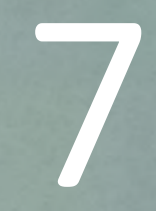

General discussion 


\section{General discussion}

The main aim of this dissertation is to increase the knowledge about the meaningful* use of activity trackers in healthcare for (older) adults with chronic diseases and for older adults with or without chronic diseases. Three phases can be distinguished within this dissertation (Figure 6.1).

Meaningful use of activity trackers in healthcare Towards a personalised use in people with a chronic disease and older adults

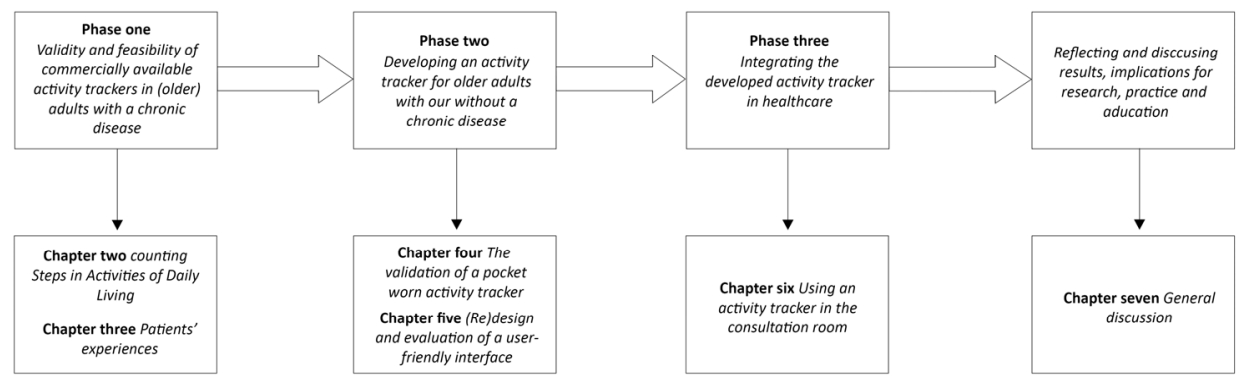

Figure 6.1 Outline of dissertation in three phases, in which five studies and seven chapters are incorporated.

Phase One focused on the validity and feasibility of commercially available activity trackers in (older) adults with a chronic disease who are generally seen in everyday physiotherapy practice. In chapters two and three, the validity and feasibility of commercially available activity trackers in (older) adults with a chronic disease are described. Using the results of phase one, an existing activity tracker was (re)designed in phase Two. The algorithm was adjusted to more validly capture activities of daily living in older adults with or without chronic diseases, and the user interface was designed in cocreation with older adults. This 'new' activity tracker was called the Measure It Super Simple (MISS) Activity. Chapter four describes the validity of the adjusted algorithm. Chapter five illustrates the co-creation process of the new user interface, as well as the feasibility of the MISS Activity tracker. In phase Three the MISS Activity was integrated into the daily clinical practice of healthcare professionals. The results of this action

\footnotetext{
* The word 'meaningful' refers to our intention that the use of an activity tracker should be personalised and incorporated into daily life and healthcare in a manner that the data support both the patient and healthcare professional. It should provide insight into the patient's activity levels, aid decisions, and support the formulation of recommendations for changing the patient's behavioural habits towards a healthier lifestyle..
} 
research study are reported in chapter six. Next, chapter seven presents an overview of the main findings of each phase, followed by general methodological considerations. Finally, the lessons learned and the study's implications for research, practice and education are given.

\section{Main findings}

The main findings are described within the three phases of the project.

\section{Phase One: Validity and feasibility of commercially available activity trackers in (older) adults with a chronic disease}

In chapter two, nine commercially available activity trackers were validated with a standardised free-living protocol in which (older) adults with a chronic disease performed activities of daily living. The step count from the activity tracker was compared with video recordings, which is the gold standard. ${ }^{1}$ The correlation between the number of steps counted by the activity trackers and the gold standard was low. All of the activity trackers showed significant systematic differences from the gold standard and both over- and underestimated step count, depending on the number of steps taken. The Fitbit One appeared to be the most valid activity tracker on a group level. On an individual level, the Fitbit One also showed large over- and underestimations of step count. A possible explanation for the low validity of these activity trackers is the generally low walking speed during activities of daily living, since studies showed that low walking speed reduces the validity of activity trackers. ${ }^{2-5}$

Next, in chapter three the feasibility of commercially available activity trackers during physiotherapy treatment in (older) adults with a chronic disease was assessed. Patients experienced the use of activity trackers as positive and motivational in terms of increasing their physical activity levels. However, they mentioned several barriers that compromised the feasibility and would have liked more guidance from their physiotherapists. The three most mentioned barriers for (older) adults with a chronic disease were as follows: the complexity of the activity tracker, 'pre-set' goals that were too high (e.g. 10.000 steps) and a lack of instructions. Furthermore, patients indicated that neither they nor their physiotherapists integrated the activity tracker into their physiotherapy sessions. Based on the results of this study, a framework was developed to examine the feasibility of activity trackers and other eHealth tools (found in the appendix to chapter three). 
The results of phase one showed that the validity and feasibility of commercially available activity trackers were insufficient for (older) adults with a chronic disease. In summary, there seemed to be a need for a feasible activity tracker that can validly measure activities of daily living in (older) adults with a chronic disease.

\section{Phase Two: Developing an activity tracker for older adults with or without chronic diseases}

In phase two, an existing activity tracker, the $\mathrm{MOX}^{6}$, was (re)designed in co-creation with older adults with or without chronic diseases. The MOX was selected as a departure point since both the algorithm and user interface were adaptable. The (re)design of the algorithm and user interface resulted in the MISS Activity. ${ }^{7}$ The algorithm was optimised with parameter settings for step count and physical behaviour of older adults with or without chronic diseases for a pocket worn activity tracker measuring activities of daily living. In chapter four, it was shown that the optimised algorithm was more valid during a non-standardised, free-living protocol with activities of daily living than generic, widely

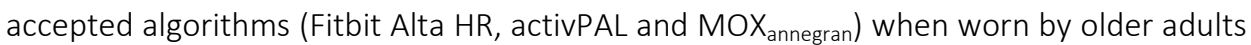
with or without chronic diseases. Next, chapter five describes the process of co-creation with older adults with or without chronic diseases and four experts in wearables (a professor in the field of smart devices, a physiotherapist, an advisor from the knowledge centre for sports and physical activity and a clinical operator of a human kinetics lab) to (re)design the user interface. This (re)design process resulted in the final design of the activity tracker (look and feel), a feedback system on the activity tracker and an app for additional feedback. Thereafter, the user interface using the optimised algorithm was tested by older adults with or without chronic diseases in their daily lives for two weeks. They experienced the MISS Activity as easy to use, thought that the additional material (e.g. manual) offered sufficient support and felt that they could therefore independently use the MISS Activity. In addition, older adults with or without chronic diseases reported that the app provided objective insight into their physical activity levels.

\section{Phase Three: Integrating the developed activity tracker into healthcare}

In phase three, the MISS Activity was integrated into daily clinical practice, together with psychosomatic therapists and patients of Het Rughuis, a specialised rehabilitation centre for people with chronic somatic symptom disorders specific to spinal pain. An action research design with several iterations was used to experience, reflect on and learn about how and when the MISS Activity could be used in a meaningful way in daily clinical practice. Therapists needed guidance from the research team to use the activity tracker in a meaningful way. This was done by composing a manual together and having several 
discussions about the meaningful use of activity trackers. During this study, the MISS Activity was mostly used to assess physical activity and to support changes in physical activity levels by setting physical activity goals. In addition, this information supported the healthcare professionals' clinical reasoning (e.g. diagnosing the coping mechanism). Prior to the recent COVID-19 regulations, therapists expressed an understanding of how to use the activity tracker in a meaningful way and planned changes to better integrate the activity tracker in their daily clinical practices. However, the proposed changes could not be fully applied due to the closing of the rehabilitation centre because of COVID-19 restrictions.

\section{Methodological considerations}

Within this section, certain overall methodological considerations are discussed: the structure of the performed phases, the selection of activity trackers, the target population, determining the validity of activity trackers and the umbrella concept 'feasibility'.

\section{The structure of the performed phases}

A strength of this dissertation is its structure; the performed phases in the different chapters form a natural sequential construct. Since little was known about the clinometric properties of commercially available activity trackers in the healthcare of (older) adults with a chronic disease and older adults with or without chronic diseases, this was assessed first. Based on the results of phase one, phase two was constructed, and the MISS Activity was developed. To use the knowledge acquired, in phase three, the MISS Activity was implemented in daily clinical practice. For each phase, the research designs were chosen based on the research question and stage, which led to a variety of research designs. A common feature of the designs was the active participation of all stakeholders during the studies. The active participation of relevant stakeholders enhances implementation in daily practice, as well as the relevance and generalisability of a product. 8,9 One limitation might be that this study did not examine the effectiveness of activity trackers in healthcare. However, the steps that were performed in this dissertation were a prerequisite to be able to study this effectiveness in future research.

\section{The selection of activity trackers}

The selection of the activity trackers in phase one was based on requirements set in collaboration with physiotherapists. After an extensive search of all commercially 
available activity trackers that were on the market in May 2015, a total of 72 trackers were found to be eligible. To ensure that the scope of different system requirements was covered, trackers were randomly selected based on the following criteria: variety of tracker location, variety of activity tracker types (e.g. accelerometer) and variety of tracked variables (e.g. steps and heart rate). By randomly selecting the activity trackers based on the above criteria, a representative sample of the range of activity trackers in $2015 / 2016$ was used to test validity and feasibility.

Since the beginning of this dissertation in 2016, the commercial market of activity trackers has exploded, with more brands, more diverse activity trackers, and trackers integrated into smartwatches and smartphones. The commercial market of activity trackers mainly targets healthy adults, athletes and people who exercise regularly. With this focus, more new variables and features were added to current activity trackers (e.g. guiding breathing sessions, playing music, enabling paying with one's activity tracker). In addition, some activity trackers that were used in this dissertation are no longer available. Therefore, one could argue that the specific results of phase one have become outdated. However, these results are still applicable because the underlying algorithms of most activity trackers have not changed or were optimised for an active population instead of (older) adults with a chronic disease or older adults with or without chronic diseases. The results reported in chapter four confirmed this statement when three more recent activity trackers were validated together with the MISS Activity. The latter proved to be more valid than the three recent activity trackers in older adults with or without chronic diseases during activities of daily living.

In contrast to these algorithms, the user interfaces of activity trackers have changed in the past five years. The first activity trackers were fairly simple to use, but adding more features to them and embedding them into smartwatches and smartphones made them more complex to use, especially for older adults. However, incorporating them into smartphones could also be useful, since it might be more appealing to start measuring physical activity with a smartphone one already owns than to buy a new activity tracker. It is to be expected that the experiences of (older) adults with chronic diseases and older adults with current, commercially available activity trackers will most likely be the same.

\section{The target population}

In this dissertation, both (older) adults with a chronic disease and older adults with or without chronic diseases participated in different phases. Within the performed studies, algorithms were validated in both target populations. There is a strong overlap between older adults and (older) adults with a chronic disease. In general, both groups have 
reduced physical activity levels and different walking patterns ${ }^{10-14}$ and rely on activities of daily living as their primary sources of physical activity. ${ }^{15}$

Within the validity studies in this dissertation, people using walking aids (e.g. a cane or walker) were exclued because they have differnt gait patterns compared to people who do not use walking aids. ${ }^{16}$ Therefore, the results from the validity studies are probably not transferable to people who use walking aids. The algorithm developed in phase two could potentially be adapted to people who use walking aids in the same way as was done for people with low walking speeds in general and/or during activities of daily living. This could be relevant for a large group of older adults and (older) adults with a chronic disease, but also for people who are recovering in hospitals or rehabilitation centres. Another target population might be 'white-collar' workers, since their occupations are generally sedentary, requiring low levels of physical activity. ${ }^{17}$

The user interface was designed according to the preferences and needs of older adults, but the main aim was to make a simple user interface. In principle, an uncomplicated user interface could be used by any target group with a preference for simplicity. Another consideration regarding the target group was that the group could have had a selection bias; participants in the studies could have been more interested in technology and possibly more skilled at using technology. This might have affected the results of the development study and feasibility studies. However, using technology is becoming more popular among older adults. In 2019, $76 \%$ of older adults were using social media, with $73 \%$ online on a daily basis and $62 \%$ using the internet to retrieve health information. ${ }^{18,19}$ Therefore, the results of the development study and feasibility study might be generalisable for a growing group of older adults, both now and for years to come.

\section{Determining the validity of activity trackers}

One strength of this dissertation is the setup of the validation study; it used not only activities of daily living, but also a widely accepted gold standard. In several other studies, activity trackers were validated in lab settings using a gold standard but not a free-living protocol with activities of daily living (e.g. using a treadmill). Alternatively, a free-living protocol was used, but validation took place without comparison to a gold standard. ${ }^{20-24}$ In the work for this dissertation, the research team simulated free living as far as possible, still using the gold standard to validate the activity trackers. The team also used other commercially available activity trackers as references, and by having several references, the validity of the activity trackers could be more accurately interpreted. One recent study proposed a protocol for determining the validity of the step count of consumer wearables and smartphones. ${ }^{1}$ Most of the criteria for this 
protocol were met in the validation studies performed for this dissertation, thus ensuring methodological and reporting consistency and facilitating comparisons between activity trackers. Three proposed activities were not included in our study: computer use, smartphone use and simulated video games. Furthermore, cadence and gait velocity were not reported.

As stated before, it is important to have insight into the validity of an activity tracker on both a group and individual level, depending on the goal of using the activity tracker. The validity on an individual level is important for the end-users (e.g. healthcare professionals or older adults), whereas validity on a group level is more important to policymakers and researchers. Two guidelines propose statistical tests when validating a measurement tool in order to create standardisation. ${ }^{25,26}$ In this dissertation both guidelines were applied to provide information on a group level as well as an individual level (state-of-the-art validation). One limitation might be that this study did not assess the reliability of the activity trackers, which is also an important clinometric property. However, due to the structure of the validation protocols used, it was not possible to determine the reliability.

\section{The umbrella concept 'feasibility'}

During the course of this dissertation, a framework was developed in which the feasibility of activity trackers and other eHealth tools can be examined (found in the appendix to chapter three). The framework is based on the literature on the feasibility of activity trackers, the findings of this team's own studies, and user and expert assessments. Feasibility is an important trait; if an activity tracker is not feasible, it will not be used persistently. However, feasibility is a broad term, referring to both patient and professional use as well as aspects of the measurement tool. It is possible that some aspects of the broad term 'feasibility' are not fully embedded in the current framework since it was evaluated within a relatively small group and a limited context. Thus, this framework is a first step to assess the feasibility of activity trackers in daily clinical practice and could be a 'dynamic framework', where aspects of feasibility in different contexts are added or adjusted as needed.

\section{Lessons learned}

The lessons that can be learned from this dissertation are described in relation to the following themes: 'involving stakeholders is the key to success', 'measuring with an 
activity tracker in healthcare is not vastly different from what healthcare professionals are used to' and 'implementation in daily practice is challenging but important'.

\section{Involving stakeholders is the key to success}

Involving stakeholders is important when creating an innovation. To illustrate this, when developing the app for the MISS Activity, it was assumed that swiping was too complicated for older adults and that pressing buttons would be an easier way for them to navigate through the app. During the initial pilot testing of the app, all older adults began swiping to navigate through the app. Despite the involvement of older adults in the look and feel of the app, this team did not ask their opinion on the navigation options of the app. This example illustrates the need to always involve stakeholders in every stage of the research. The active participation of relevant stakeholders enhances implementation in daily practice, including the relevance and generalisability of a product. $^{8,9}$

Innovative ways to retrieve information and include stakeholders are co-creation methods, of which several have been used in this dissertation. These co-creation methods are frequently used by designers but are also applicable in research. ${ }^{27}$ Beyond retrieving information, these methods are enjoyable to perform and provide an opportunity for participants to get to know each other's ideas and find common ground regarding terminology, which is essential for a successful interdisciplinary collaboration. $^{28}$

To identify all relevant stakeholders in this dissertation, 'stakeholder mapping' was used. ${ }^{29}$ Stakeholders can participate in all stages of the research and in different roles, which can vary at each stage. These roles can range from only informing stakeholders to giving them full control. ${ }^{30}$ To define which role suits which stakeholder best, the 'participation matrix' was used. ${ }^{30}$ Within this dissertation, the stakeholders participated to the full extent of the participation matrix. Other co-creation methods that were also used in this dissertation included the Moscow method, the business model canvas, prototyping and the six thinking hats. ${ }^{31-34}$

To choose and perform the right methods, researchers need adequate support and training. ${ }^{9,35}$ This PhD trajectory was part of the Brightlands Innovation Programme 'Limburg Meet' (LIME), a programme that facilitates smarter measurement methods and more efficient data collection for better care and health. ${ }^{36}$ Part of this programme was a co-creation team, with two co-creation experts, that supported the research teams. Moreover, a collaborating network of researchers, entrepreneurs, civilians and educators was developed within the LIME programme. This allowed us to collaborate with several different disciplines throughout the course of this dissertation. For example, 
a hundred students from the education communication and multimedia design study participated in this research during one of their course modules, in which they had to work on a real-life case in groups of four-five students. They developed paper prototypes of the user interfaces for the app for the MISS Activity in collaboration with older adults and researchers from Zuyd University of Applied Sciences and industrial designers from Maastricht University. After collaborating with ICT students and ICT experts from Maastricht University, the former students' designs were partly used in the final design of the MISS Activity.

\section{Measuring with activity trackers in healthcare is not vastly different from what healthcare professionals are used to}

There are numerous measurement tools in healthcare that can be used to assess aspects of health. Several guidelines have been developed to assist healthcare professionals in choosing and using a relevant measurement tool for their purposes. ${ }^{37-39}$ They all advise establishing 'why' and 'what' one wants to measure, what the clinometric properties of the chosen measurement tool are, and how to interpret and use the data from the measurement tool. The same conditions apply when using an activity tracker in daily clinical practice; that is, choosing and using an activity tracker does not differ from employing any other measurement tool. However, healthcare professionals require some new eHealth competencies in order to use eHealth. ${ }^{40,41}$ Barakat et al. formulated five competency themes: ICT attitudes and skills, interpretation and analysis of eHealth data, support and guidance, communication skills and privacy and confidentiality. ${ }^{40}$ Current healthcare professionals are not trained in all of these competencies and therefore struggle to perform these skills in their daily clinical practice. For example, patients expressed the need for more guidance from their physiotherapists to fully use the activity tracker (phase one, chapter three). The skill 'the healthcare professional educates end-users in the operation and functionality of the technology' is required to support patients when asked. In phase three, the healthcare professionals and the research team often discussed topics related to the skills 'effectively combine clinical knowledge with eHealth data in decision-making' and 'translate the data effectively within the context of the client'. These skills are also required when using 'regular' measurement tools. ${ }^{37}$ For both 'regular' measurement tools and activity trackers, the same process is employed in deciding, choosing and using the measurement tool. Healthcare professionals require support and training to connect their existing skills to the use of activity trackers and the new eHealth competencies required, such as ICT skills and privacy and confidentiality. Healthcare education, postgraduate continuing professional education and master courses should implement these competencies in 
their education programmes since the use of eHealth in healthcare is rising, and the subject is given limited attention in the current curricula for healthcare professionals. ${ }^{42-44}$ To implement more eHealth in these curricula, a first step might be to teach educators about the meaningful use of eHealth and the similarities with 'regular' measurement tools.

\section{Implementation in daily practice is challenging but important}

During this PhD trajectory, the research team learned that healthcare professionals need more support in implementing activity trackers than was foreseen. Several reports on the implementation of eHealth explain that the bottleneck is the transition from the pilot phase to implementation and upscaling in daily clinical practice. ${ }^{45,46}$ For an innovation to be successfully implemented, the eHealth tool should fit the daily clinical practice, but healthcare professionals should also be open to change. ${ }^{45}$ To achieve these goals, a systematic implementation approach is recommended, such as that of Grol et al., $^{47}$ the Consolidated Framework For Implementation Research (CFIR) ${ }^{48}$ and usercentred design. ${ }^{49}$ Important features of these approaches are the thorough problem analyses (Grol et al.) and the use of an iterative approach (user-centred design). However, there is a range of implementation methods and strategies. Therefore, it is important but difficult to choose a method that fits the implementation phase, context and stakeholders. ${ }^{50}$ Moreover, there are several barriers to and facilitators of implementation that must be considered, ${ }^{48}$ some specific to the implementation of eHealth. ${ }^{51,52}$ To embed all of these aspects in one implementation design is challenging, which was evident during this study's action research design. It is important to maintain a balance between support from the research team and time for healthcare professionals to experience the use of activity trackers. On the one hand, healthcare professionals can learn from good examples and studies showing that they need help to understand how to use eHealth and recognise its benefits. ${ }^{51,53,54}$ On the other hand, to sufficiently implement an activity tracker, healthcare professionals must experience what works and what does not work in their own daily clinical routines. This team learned that dialoguing with healthcare professionals about their theoretical frameworks, routines, and dilemmas, including examples, helps professionals understand how and when to use the activity trackers. By using an action research design and including both healthcare professionals and patients, the implementation of the activity tracker had a better chance of succeeding. ${ }^{55,56} \mathrm{~A}$ limitation within our action research design might be that we did not base the approach on existing theories such as behaviour change theories. 
The implementation of eHealth in healthcare might be more urgent now than in past years. Due to COVID-19 pandemic measures, a major part of healthcare has recently been remotely performed. Providing healthcare remotely also means measuring remotely, and the COVID-19 pandemic has proved to be a huge driver of the implementation of eHealth. ${ }^{57}$ To create sustained use, the focus should be on embedding eHealth in the process of daily care and clinical reasoning. Current healthcare systems face challenges in keeping healthcare sustainable. It is predicted that by 2040 there will be 9.8 million people with chronic diseases ${ }^{58}$ and 1.6 million people who are 80 years or older ${ }^{59}$ in the Netherlands. eHealth, including activity trackers, could be part of the solution to keeping healthcare sustainable. It has the potential to reduce workload, support self-management of patients, and monitor and analyse patients to proactively intervene. ${ }^{46,60,61}$

\section{Implications for research, education and practice}

The following section describes the implications of the results of this dissertation for research, education and practice to facilitate the meaningful use of activity trackers in healthcare.

\section{Implications for research}

For researchers in the field of activity trackers and health technology, it is recommended that they:

- Focus on further research on the implementation of activity trackers in daily clinical practice with the active involvement of all stakeholders to the full extent of the participation roles (informing, consultation, placation, partnership, delegation or citizen control) by using co-creation methods and participatory action research designs.

- Use the knowledge and expertise of healthcare professionals regarding daily routines and clinical reasoning when implementing innovations such as activity trackers in their daily clinical practice.

- Be aware of the limited validity and feasibility of current commercially available activity trackers when using them to measure activities of daily living in studies of older adults with or without chronic disease and (older) adults with a chronic disease. 
- Validate and test the feasibility of commercially available activity trackers in more target populations, such as people who use walking aids, patients in rehabilitation centres and hospitals and inactive adults.

- Use the developed feasibility framework to evaluate aspects of the feasibility of activity trackers in different contexts and consider using the results to further develop the feasibility framework.

- Perform effectiveness studies with activity trackers when activity trackers are fully implemented in daily clinical practice.

- Consider using the MISS Activity in research when measuring physical activity in populations in which low walking speed is evident generally or during activities of daily living, and/or in populations for which simple technical tools are desirable (e.g. in older adults with or without chronic disease and (older) adults with a chronic disease).

\section{Implications for education}

For developers and teachers of educational programmes for healthcare students and healthcare professionals, it is recommended that they:

- Teach healthcare students and professionals the required new eHealth competencies, allowing them to independently choose and use eHealth devices such as activity trackers in clinical practice.

- Teach healthcare students and professionals the similarities between eHealth measurement tools, such as activity trackers, and 'regular' measurement tools.

- Provide good examples to healthcare students and professionals to illustrate how to use activity trackers in their daily clinical practices as support tools for their clinical reasoning.

- Stimulate interdisciplinary collaboration, including co-creation sessions, to develop strong collaborations and insight into each other's competencies, with all students involved in the development and use of an eHealth tool.

- Invest in and develop training where healthcare professionals can experience, reflect and learn with each other about when and how to use activity trackers as support tools in daily clinical practice. This could be done through on-the-job coaching, in field labs or learning communities. 


\section{Implications for practice}

For healthcare professionals, it is recommended that they:

- Consider using an activity tracker in daily clinical practice to support clinical reasoning and, if applicable, combine the activity tracker with other relevant measurement tools.

- Follow a training programme to learn to use an activity tracker meaningfully in daily clinical practice.

- Be aware of the limited validity of commercially available activity trackers during activities of daily living.

- Use the feasibility framework to choose together with the patient an activity tracker that matches the patient's skills and requirements.

- Consider using the MISS Activity when matching with the healthcare professional's and patient's requirements and skills.

- Provide more support in terms of instructions and problem-solving for patients with limited technical skills and eHealth literacy when using an activity tracker in daily clinical practice.

- Use an activity tracker to assess and monitor the physical activity level of a patient and, if applicable, to change the physical activity level, using the following goals: provide more physical activity during the day, provide less physical activity during the day or divide physical activity during the day.

- Learn from and with colleagues and patients when implementing an activity tracker or other eHealth tools. 


\section{References}

1. Johnston W, Judice PB, Molina García P, et al. Recommendations for determining the validity of consumer wearable and smartphone step count: expert statement and checklist of the INTERLIVE network. British journal of sports medicine 2020. Online ahead of print.

2. Floegel TA, Florez-Pregonero A, Hekler EB, Buman MP. Validation of Consumer-Based Hip and Wrist Activity Monitors in Older Adults With Varied Ambulatory Abilities. J Gerontol A Biol Sci Med Sci. 2017;72(2):229-236.

3. Beevi FH, Miranda J, Pedersen CF, Wagner S. An Evaluation of Commercial Pedometers for Monitoring Slow Walking Speed Populations. Telemed J E Health 2016; 22(5): 441-9.

4. Martin JB, Krč KM, Mitchell EA, Eng JJ, Noble JW. Pedometer accuracy in slow walking older adults. Int J Ther Rehabil 2012; 19(7): 387-93.

5. Cyarto EV, Myers A, Tudor-Locke C. Pedometer accuracy in nursing home and community-dwelling older adults. Med Sci Sports Exerc 2004; 36(2): 205-9.

6. Maastricht Instruments BV. MOX2. URL: http://www.accelerometry.eu/mox2/ (accessed 2021-02-16).

7. Maastricht Instruments BV. MISS Activity. URL: https://www.accelerometry.eu/miss-activity/ (accessed 2021-02-16).

8. Gradinger F, Britten N, Wyatt K, et al. Values associated with public involvement in health and social care research: a narrative review. Health Expect 2015; 18(5): 661-75.

9. van Dijk-de Vries A, Stevens A, van der Weijden T, Beurskens A. How to support a co-creative research approach in order to foster impact. The development of a Co-creation Impact Compass for healthcare researchers. PloS one 2020; 15(10).

10. Lahousse L, Verlinden VJ, van der Geest JN, et al. Gait patterns in COPD: the Rotterdam Study. The European respiratory journal 2015; 46(1): 88-95.

11. Mueller MJ, Minor SD, Sahrmann SA, Schaaf JA, Strube MJ. Differences in the Gait Characteristics of Patients With Diabetes and Peripheral Neuropathy Compared With Age-Matched Controls. Physical Therapy 1994; 74(4).

12. Kaufman KR, Hughes C, Morrey BF, Morrey M, An KN. Gait characteristics of patients with knee osteoarthritis. J Biomech 2001; 34(7): 907-15.

13. Pirker W, Katzenschlager R. Gait disorders in adults and the elderly : A clinical guide. Wien Klin Wochenschr 2017; 129(3-4): 81-95.

14. Verlinden VJ, van der Geest JN, Hoogendam YY, Hofman A, Breteler MM, Ikram MA. Gait patterns in a community-dwelling population aged 50 years and older. Gait Posture 2013; 37(4): 500-5.

15. Hildebrandt VH, Bernaards CM, Hofstetter H. Trendrapport Bewegen en Gezondheid 2000/2014. $1^{\text {ste }}$ edition. Ridderkerk: TNO: Hollandridderkerk; 2014.

16. Mundt M, Batista JP, Markert B, Bollheimer C, Laurentius T. Walking with rollator: a systematic review of gait parameters in older persons. Eur Rev Aging Phys Act 2019; 16: 15.

17. Smith L, McCourt O, Sawyer A, et al. A review of occupational physical activity and sedentary behaviour correlates. Occup Med (Lond) 2016; 66(3): 185-92.

18. Centraal Bureau voor Statistiek. Internet; toegang, gebruik en faciliteiten. URL: https://opendata.cbs.nl/statline/\#/CBS/nl/dataset/83429NED/table?ts=1586173445506 (accessed 202102-16).

19. Centraal Bureau voor Statistiek. ICT-gebruik van huishoudens en personen. URL: https://longreads.cbs.nl/ict-kennis-en-economie-2019/ict-gebruik-van-huishoudens-en-personen/ (accessed 2021-02-16).

20. Evenson KR, Goto MM, Furberg RD. Systematic review of the validity and reliability of consumerwearable activity trackers. Int J Behav Nutr Phys Act 2015; 12: 159.

21. Straiton $\mathrm{N}$, Alharbi $\mathrm{M}$, Bauman $\mathrm{A}$, et al. The validity and reliability of consumer-grade activity trackers in older, community-dwelling adults: A systematic review. Maturitas 2018; 112: 85-93.

22. Fuller D, Colwell E, Low J, et al. Reliability and Validity of Commercially Available Wearable Devices for Measuring Steps, Energy Expenditure, and Heart Rate: Systematic Review. JMIR Mhealth Uhealth 2020; 8(9): e18694. 
23. Van Remoortel H, Giavedoni S, Raste $\mathrm{Y}$, et al. Validity of activity monitors in health and chronic disease: a systematic review. Int J Behav Nutr Phys Act 2012; 9: 84.

24. Gorzelitz J, Farber C, Gangnon R, Cadmus-Bertram L. Accuracy of Wearable Trackers for Measuring Moderate- to Vigorous-Intensity Physical Activity: A Systematic Review and Meta-Analysis. Journal for the Measurement of Physical Behaviour 2020; 3(4): 346-57.

25. Welk GJ, Bai Y, Lee JM, Godino J, Saint-Maurice PF, Carr L. Standardizing Analytic Methods and Reporting in Activity Monitor Validation Studies. Med Sci Sports Exerc 2019; 51(8): 1767-80.

26. Mokkink LB. COSMIN Risk of Bias checklist. URL: https://www.cosmin.nl/wp-content/uploads/COSMINRoB-checklist-V2-0-v17_rev3.pdf (accessed 2021-02-16).

27. Voorberg WH, Bekkers VJJM, Tummers LG. A Systematic Review of Co-Creation and Co-Production: Embarking on the social innovation journey. Public Management Review 2014; 17(9): 1333-57.

28. Nancarrow SA, Booth A, Ariss S, Smith T, Enderby P, Roots A. Ten principles of good interdisciplinary team work. Hum Resour Health 2013; 11: 19.

29. Stinkdorn M. This is service design doing. 1st edition. Sebastopol,USA: O'Reilly media; 2018. ISBN: 1491927186

30. Arnstein SR. A Ladder Of Citizen Participation. Journal of the American Institute of Planners 1969; 35(4): 216-24.

31. Blomkvist J, Holmlid S. Service Prototyping According to Service Design Practitioners. ExChanging Knowledge; 2010 1-3 Dec; Linköping; Sweden.

32. Osterwalder A. Business Model Generation. 1ste edition. New Yersey, USA: John Wiley \& Sons inc; 2010. ISBN: 9780470876411

33. Clegg D, Barker R. Case Method Fast-Track: A RAD Approach. 1t $^{\text {st }}$ edition. Boston: Addison-Wesley; 2004. ISBN: 978-0201624328

34. de Bono E. Six Thinking Hat. $1^{\text {st }}$ edition. London: Penguin Books Ltd; 2000. ISBN: 978-0140137842

35. de Wit M, Beurskens A, Piskur B, Stoffers E, Moser A. Preparing researchers for patient and public involvement in scientific research: Development of a hands-on learning approach through action research. Health Expect 2018; 21(4): 752-63.

36. Limburg Meet. Limburg Meet. URL: https://www.limeconnect.nl/. (accessed 2021-02-16)

37. Beurskens A, Swinkels R, Wittink H, van Peppen R, Vermeulen H. Meten in de praktijk. $1^{\text {st }}$ edition Houten; Bohn Stafleu van Loghum; 2020. ISBN: 9789036824583

38. van der Wees PJ, Verkerk EW, Verbiest MEA et al. Development of a framework with tools to support the selection and implementation of patient-reported outcome measures. Journal of Patient-Reported Outcomes. 2019; 30;3(1):75.

39. Aaronson N, Elliott T, Greenhalgh J, et al. User's Guide to Implementing Patient-Reported Outcomes Assessment in Clinical Practice. URL: https://www.isoqol.org/wp-content/uploads/2019/09/ 2015UsersGuide-Version2.pdf. (accessed 2021-02-16)

40. Barakat A, Woolrych RD, Sixsmith A, Kearns WD, Kort HS. eHealth Technology Competencies for Health Professionals Working in Home Care to Support Older Adults to Age in Place: Outcomes of a Two-Day Collaborative Workshop. Med 20 2013; 2(2): e10.

41. Brunner M, McGregor D, Keep M, et al. An eHealth Capabilities Framework for Graduates and Health Professionals: Mixed-Methods Study. J Med Internet Res 2018; 20(5): e10229.

42. dirippulige $\mathrm{S}$, Brooks $\mathrm{P}$, Carati $\mathrm{C}$, et al. It's important, but not important enough: eHealth as a curriculum priority in medical education in Australia. J Telemed Telecare 2018; 24(10): 697-702.

43. Wentink MM, Siemonsma PC, van Bodegom-Vos L, et al. Teachers' and students' perceptions on barriers and facilitators for eHealth education in the curriculum of functional exercise and physical therapy: a focus groups study. BMC Med Educ 2019; 19(1): 343.

44. Vader M. eHealth in Dutch physiotherapy practices: a national survey. Utrecht: Utrecht University; 2017.

45. Flim C. Actieplan ehealth implementatieonderzoek. URL: http://www.invoorzorg.nl/docs/ivz/ informatiecentrum/23505.pdf (accessed 2021-02-16)

46. Wouters M, Huygens M, Voogdt H, et al. E-health monitor: Samen aan zet!: Nictiz and Nivel, 2019.

47. Grol R, Wensing M, Eccles M, Davis D. Improving Patient Care. $2^{\text {nd }}$ edition. New Yersey, USA: WileyBlackwell, 2013. ISBN: 978-1-118-52597-5 
48. Damschroder LJ, Aron DC, Keith RE, Kirsh SR, Alexander JA, Lowery JC. Fostering implementation of health services research findings into practice: a consolidated framework for advancing implementation science. Implement Sci 2009; 4: 50.

49. Gulliksen J, Göransson B, Boivie I, Blomkvist S, Persson J, Cajander Å. Key principles for user-centred systems design. Behaviour \& Information Technology 2003; 22(6): 397-409.

50. Wensing M, Grol R. Knowledge translation in health: how implementation science could contribute more. BMC medicine 2019; 17(1): 88.

51. Schreiweis B, Pobiruchin M, Strotbaum V, Suleder J, Wiesner M, Bergh B. Barriers and Facilitators to the Implementation of eHealth Services: Systematic Literature Analysis. J Med Internet Res 2019; 21(11): e14197.

52. Gagnon MP, Ngangue P, Payne-Gagnon J, Desmartis M. m-Health adoption by healthcare professionals: a systematic review. J Am Med Inform Assoc 2016; 23(1): 212-20.

53. Ariens LF, Schussler-Raymakers FM, Frima C, et al. Barriers and Facilitators to eHealth Use in Daily Practice: Perspectives of Patients and Professionals in Dermatology. J Med Internet Res 2017; 19(9): e300.

54. Ross J, Stevenson F, Lau R, Murray E. Factors that influence the implementation of e-health: a systematic review of systematic reviews (an update). Implement Sci 2016; 11(1): 146.

55. Koshy E, Koshy V, Waterman H. Action Research in Healthcare. London: SAGE publications Ltd., 2011. ISBN: 978-1848601895

56. Pluut B. Actieonderzoek in vogelvlucht Resultaten van een verkenning voor ZonMw. URL: https://pluutpartners.nl/wp-content/uploads/2020/06/Actieonderzoek-in-vogelvlucht.pdf. _ (accessed 2021-02-16)

57. Raad voor volksgezondheid. Zorg op afstand dichterbij? Digitale zorg na de coronacrisis. 2020. URL: https://www.raadrvs.nl/documenten/publicaties/2020/08/27/zorg-op-afstand-dichterbij. _ (accessed 2021-02-16)

58. Menting J, van Schelven F, Grosscurt R, Spreeuwenberg P, Heijmans M. Zorgmonitor 2019. URL: https://www.nivel.nl/sites/default/files/bestanden/1003626.pdf (accessed 2021-02-16)

59. Centraal Bureau voor Statistiek. Prognose: 19 miljoen inwoners in 2039. URL: https://www.cbs.nl/nlnl/nieuws/2019/51/prognose-19-miljoen-inwoners-in-2039 (accessed 2021-02-16).

60. Rijksoverheid. Voordelen e-health. URL: https://www.rijksoverheid.nl/onderwerpen/e-health/voordelene-health (accessed 2021-02-16).

61. Barbabella F, Melchiorre MG, Quattrini S, Papa R, Lamura G. How can eHealth improve care for people with multimorbidity in Europe? European Observatory on Health Systems and Policies; 2017. 


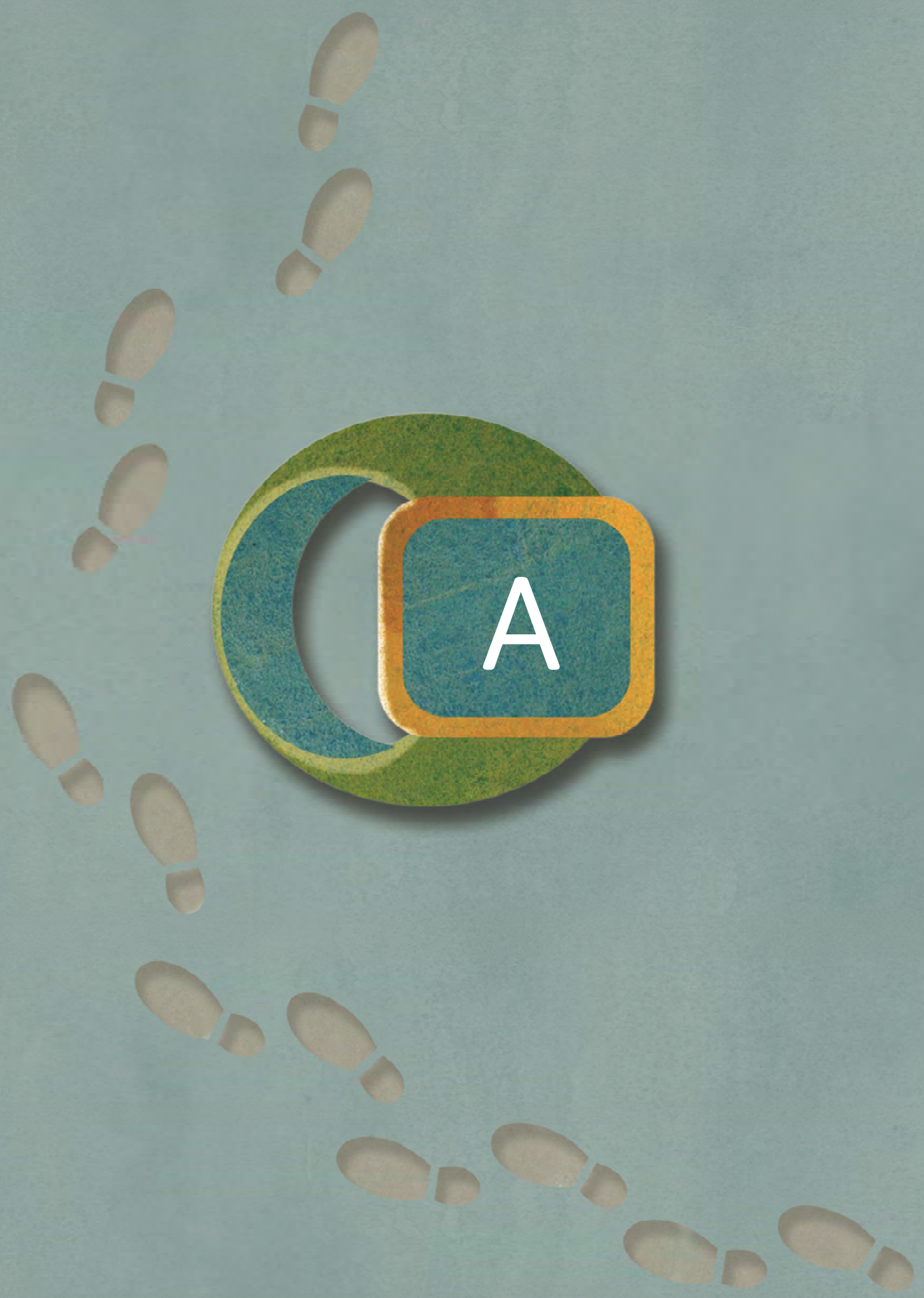




\section{English summary}

Samenvatting

Impact paragraph

Dankwoord

Curriculum Vitae 


\section{English summary}

Physical activity is key to preventing chronic disease and enabling participation in activities of daily living. Healthcare professionals, such as physiotherapists, occupational therapists, psychosomatic therapists, and nurse practitioners, often measure the physical activity levels of their patients, including older adults and people with chronic diseases. Currently, healthcare professionals use questionnaires and diaries to gain insight into their patients' physical activity levels. However, most questionnaires and diaries lack validity, reliability, and feasibility. A potential alternative method of measuring physical activity, both within and beyond the context of healthcare, is to use activity trackers, which are known to provide objective data and are feasible in use in a healthy population. However, there is a gap of evidence on both the validity and feasibility of activity trackers for those with slower walking speeds and activity levels, such as (older) adults with or without chronic diseases which are often seen in healthcare. Furthermore, no guidelines have been developed or studies performed to aid healthcare professionals in using activity trackers in their daily clinical practices. Therefore, the primary aim of this dissertation was to increase the knowledge about the meaningful* use of activity trackers in healthcare, particularly for (older) adults with chronic diseases and older adults with or without chronic diseases.

To achieve this objective, this dissertation was divided into three phases. In phase one, the validity and feasibility of commercially available activity trackers were assessed in (older) adults with chronic diseases. In phase two, an existing activity tracker was (re)designed for older adults with or without chronic diseases by adjusting the tracker's algorithm and upgrading its user interface. The 'new' activity tracker was called 'The Measure It Super Simple (MISS)' Activity. The feasibility of the MISS Activity was evaluated among older adults with or without a chronic disease. In phase three the MISS Activity was integrated into daily clinical practice.

Chapter One begins by stressing the importance of physical activity and physical capacity to one's possebilities to fully participate in daily life. The chapter then delineates how healthcare professionals can measure physical activity by using subjective measurement tools (questionnaires and diaries) or objective measurement tools (doubly labelled water or indirect calorimetry). Furthermore, the advantages and disadvantages of these

\footnotetext{
* The word 'meaningful' refers to our intention that the use of an activity tracker should be personalised and incorporated into daily life and healthcare in a manner that the data support both the patient and healthcare professional. It should provide insight into the patient's activity levels, aid decisions, and support the formulation of recommendations for changing the patient's behavioural habits towards a healthier lifestyle.
} 
measurement tools are discussed. Thereafter, the potential use of activity trackers in healthcare is discussed, as is the aforementioned validity, and feasibility. Finally, the lack of knowledge regarding the meaningful use of activity trackers in healthcare is outlined. At the end of the first chapter, the objective, research questions, and outline of this thesis are described.

Chapter Two describes the procedure and results of a validation study. This study aimed to investigate the validity of nine commercially available activity trackers for measuring step count during activities of daily living in people who have a chronic disease and are receiving physiotherapy. The selected activity trackers were the Accupedo (Corusen LLC), Activ8 (Remedy Distribution Ltd), Digi-Walker CW-700 (Yamax), Fitbit Flex (Fitbit inc), Lumoback (Lumo Bodytech), Moves (ProtoGeo Oy), Fitbit One (Fitbit inc), UP24 (Jawbone), and Walking Style X (Omron Healthcare Europe BV). In total, 130 (older) adults with chronic diseases performed a standardised activity protocol, based on activities of daily living. The protocol was recorded on camera, and their step count was analysed through video observations (gold standard). The validity of the trackers' step count was assessed by correlation coefficients, t-tests, scatterplots, and Bland-Altman plots. The correlations between the number of steps counted by the activity trackers and the gold standard were low (range: -0.02 to 0.33 ). For all activity trackers except the Fitbit One, a significant systematic difference with the gold standard was found for the step count. Plots showed a wide range in scores and an average underestimation was found for all activity trackers, except for the Activ8, for which an average overestimation was found. In conclusion, this study showed that the validity of nine commercially available activity trackers is low regarding their capacity to measure the step count of (older) adults who have chronic diseases, are receiving physiotherapy, and are engaged in activities of daily living.

Chapter Three illustrates the experience with commercially available activity trackers within the physiotherapeutic treatment of (older) adults with chronic diseases. The qualitative study in question included twenty-nine participants with a chronic disease. Each participant wore one of the following activity trackers: Accupedo (Corusen LLC), Activ8 (Remedy Distribution Ltd), Digi-Walker CW-700 (Yamax), Fitbit Flex (Fitbit inc), Lumoback (Lumo Bodytech), Moves (ProtoGeo Oy), Fitbit One (Fitbit inc), UP24 (Jawbone), and Walking Style X (Omron Healthcare Europe BV) throughout the day for two to eight weeks. Data were collected using twenty-three individual interviews as well as a focus group with six participants. A framework analysis with deductive and inductive content analyses was used to analyse the data. The framework analysis produced seven categories: purchase, instruction, characteristics, correct functioning, sharing data, 
privacy, use, and interest in feedback. Although the tracker motivated the participants to be more active, participants experienced the standard step count goal of the activity trackers as 'too high' and the tracker itself as too complex. They reported that they would have liked more guidance from their physiotherapists regarding the use of the trackers. Furthermore, the participants experienced some technical failures, were willing to share data with their physiotherapists, and wanted to spend an average of maximum fifty euros on an activity tracker. If activity trackers are to be embedded in physiotherapy, more attention should be paid to their integration into treatment and to helping (older) adults with a chronic disease use and interpret their data. The developed framework offered insight into each of the key concepts reported by patients with a chronic disease and can be used to guide further research and practices.

Chapter Four presents the results of a study, which had two purposes: to (re)design the user interface of an existing activity tracker (so-called MOX), considering the input of older adults with or without chronic diseases living independently, and to assess the daily use of and experiences with the adapted activity tracker the so-called 'Measure It Super Simple' (MISS) Activity tracker.

The double diamond method was used to (re)design the user interface. As a departure point, this study used a list of general design requirements that facilitate the development of technology for older adults. In co-creation with thirty-five older adults, the design, feedback system, and application were further developed into a user friendly interface: the 'Measure It Super Simple' (MISS) Activity.

Second, the use of and experiences with the adapted MISS Activity in daily life were assessed. Twenty-eight older adults used the MISS Activity for two weeks. Their user experiences were assessed through interviews. The participants reported that the MISS Activity was easy to use; they needed limited help when setting up the tracker and required limited assistance when using it throughout their daily lives. The participants also reported that they thought that anyone should be able to use it. This study offers a generic structured methodology and a list of design requirements, which can be used to adapt the interface of an existing activity tracker to suit the skills and needs of older adults with or without a chronic disease. The MISS activity seemed to be successfully (re)designed.

Chapter Five describes the validation of optimised algorithm parameter settings for step count and physical behaviour of a pocket-worn activity tracker used by older adults with our without chronic diseases during activities of daily living $\left(\mathrm{MOX}_{\text {MissActivity }}\right)$. For a more relevant interpretation of the results, the performance of the MOX $_{\text {Missactivity }}$ was compared to three reference applications: the $\mathrm{MOX}_{\text {Annegran, }}$ the activPAL, and the Fitbit 
Alta HR. In a cross-sectional validation study, twenty older adults performed an activity protocol based on activities of daily living. Their performance was video recorded and analysed for step count and dynamic, standing, and sedentary time. Validity was assessed by percentage error (PE), absolute percentage error (APE), Bland-Altman plots,

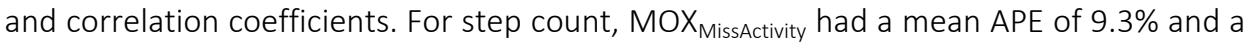
correlation coefficient of 0.88 . The mean APE values of dynamic, standing and, sedentary time were $15.9 \%, 19.9 \%$, and $9.6 \%$, respectively. The correlation coefficients were 0.55 ,

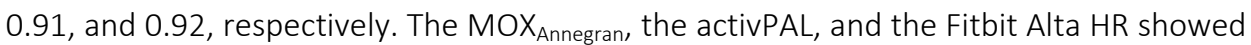
higher errors and lower correlations for all outcome variables. This study showed that the optimised algorithm parameter settings can more validly estimate step count and physical behaviour during activities of daily living of older adults with our without chronic diseases wearing an activity tracker in their trouser pocket, than reference applications can.

In Chapter Six, the MISS Activity was embedded in daily clinical practice to aid the clinical reasoning of healthcare professionals, to facilitate the engagement of patients in their treatment, and to enhance knowledge about the implementation process in clinical practice. The study was performed in a specialised rehabilitation centre for people with chronic somatic symptom disorders specific to spinal pain, and both healthcare professionals (psychosomatic therapists) and patients participated. An action research design was used so that psychosomatic therapists and patients could experience, reflect on, and learn about how and when they can use activity trackers. This process used as a departure point a draft manual, which the therapist adapted during the iterations to their specific context. Data collection was performed with audio recordings of conversations about the activity trackers during therapy, reflection sessions with the therapists with support from the research team, and semi-structured individual interviews with the patients. Analyses were performed by directed content analyses based on the coding framework of Chapter Three. In total, three therapists and eleven patients participated. Twenty-eight conversations during therapy about the measurement of physical activity, eleven semi-structured interviews, and four reflection sessions were recorded within three iteration sessions. Throughout the iterations, therapists continued to develop the manual in which their theoretical framework and clinical reasoning were integrated. To achieve adequate reflection and depth during the reflection sessions, therapists needed a considerable amount of guidance from the research team. They also required sufficient time and sufficient patients to build up a knowledge base upon which to act. The therapists formulated exclusion criteria for patients as well as a flowchart clarifying when the activity tracker should be used, how to define goals, and how to discuss the (data of) the activity tracker. Not only did this action 
research approach and its multiple iterations helped therapists learn and reflect together but also embed the activity trackers into their clinical practice. In addition to demonstrating how to use an activity tracker in a meaningful way in healthcare, this study can also serve as an illustration of how to implement an eHealth tool in daily clinical practice.

Chapter Seven describes the primary findings of each of the three phases of this dissertation. Subsequently, the following methodological considerations are discussed: 1. the strength of the dissertation structure and study design; 2 . the selection process and the relevance of the selected activity trackers; 3 . the limitations and possible biases of the target populations; 4 . the strengths and weaknesses of the methodology used to validate the activity trackers; 5 . the umbrella term 'feasibility,' including the developed feasibility framework.

Furthermore, the lessons learned during the dissertation are described, including the insight that the involvement of stakeholders can be considered as the key to success, and several methods of involving these stakeholders are outlined. Hence, it is recognised that the use of activity trackers in healthcare is not totally different from what healthcare professionals are used to, since healthcare professionals already possess several of the skills required to successfully use trackers in their practice, but they must develop additional eHealth competencies if they hope to fully realise this integration. The final section of the study discusses the implementation of trackers in daily clinical practice, acknowledges the ways in which it is both challenging and important, and outlines several methods of achieving implementation.

Finally, implications for future research, education, and practice are provided. Among such implications, it is argued that subsequent research should focus on enhancing the implementation of activity trackers in daily clinical practice and should actively engage the full participation of all stakeholders by using co-creation methods and participatory designs. Furthermore, education should focus on teaching healthcare students and professionals necessary eHealth competencies to enable the independent use and conscious selection of eHealth and mHealth tools such as activity trackers in their clinical practice. In conclusion, it is argued that healthcare professionals should consider using an activity tracker in their daily clinical practice to support their clinical reasoning and, if possible, supplement the activity tracker with other relevant measurement tools 


\section{Samenvatting}

Om te kunnen participeren in het dagelijks leven en om chronische ziekten te voorkomen, is voldoende fysieke activiteit essentieel. Zorgprofessionals zoals fysiotherapeuten, ergotherapeuten, psychologen en verpleegkundigen meten bij hun patiënten regelmatig de fysieke activiteit. Vooral bij ouderen of mensen met een of meerdere chronische aandoeningen is dit relevant. Meestal wordt aan de patiënten gevraagd om een vragenlijst in te vullen of in een dagboek bij te houden hoeveel ze bewegen en welke soort activiteiten ze uitvoeren. Uit onderzoeken blijkt dat deze manieren van meten niet goed genoeg zijn (onvoldoende valide en betrouwbaar) en ook niet praktisch in het gebruik (hanteerbaarheid). Een andere manier om fysieke activiteit te meten is met behulp van een activiteitenmeter.

Om activiteitenmeters op een betekenisvolle manier te gebruiken is het belangrijk om te weten hoe valide en hanteerbaar ze zijn voor de doelgroep en hoe ze het beste kunnen gebruikt kunnen worden in de zorg. Daarom was het hoofddoel van dit proefschrift het vergroten van de toegepaste kennis over het betekenisvol gebruiken van activiteitenmeters in de gezondheidszorg, met name bij ouderen en mensen met een chronische aandoening.

Om deze doelstelling te behalen is dit proefschrift opgedeeld in drie delen. Het eerste deel gaat over de validiteit en hanteerbaarheid van commercieel verkrijgbare activiteitenmeters bij mensen met een chronische aandoening. In het tweede deel is een bestaande activiteitenmeter (door)ontwikkeld met en voor ouderen met of zonder chronische aandoening. Hiervoor werden het algoritme en de lay-out van een bestaande activiteitenmeter aangepast. Deze nieuwe activiteitenmeter kreeg de naam: 'Meten Is Super Simpel' (MISS) Activity. De validiteit en hanteerbaarheid van de MISS Activity werd geëvalueerd met thuiswonende ouderen met of zonder chronische aandoening. In het derde deel is onderzoek gedaan naar de integratie van de MISS Activity in het klinisch handelen van zorgprofessionals.

Hoofdstuk één begint met het beschrijven van het belang van fysieke activiteit en fysieke capaciteit om te kunnen participeren in het dagelijks leven. Fysieke activiteit wordt gedefinieerd als 'elke krachtsinspanning van spieren resulterend in méér energiegebruik dan in rustende toestand'. Fysieke capaciteit wordt gedefinieerd als 'de mogelijkheid om fysieke activiteit uit te voeren' en bestaat uit cardiovasculaire capaciteit, musculaire

\footnotetext{
*Het woord 'betekenisvol' refereert naar onze intentie dat het gebruik van een activiteitenmeter altijd gepersonaliseerd en geïntegreerd moet zijn in het dagelijks leven en de zorg, op een manier waarop de data zowel de patiënt als de zorgprofessional ondersteunen. De data moeten inzicht geven in de fysieke activiteit van een patiënt, ondersteuning bieden bij het maken van keuzes en ondersteunen bij gedragsverandering.
} 
capaciteit en balans. Vervolgens wordt beschreven dat zorgprofessionals voornamelijk bij ouderen en mensen met een chronische aandoening de fysieke activiteiten meten. Fysieke activiteit kan worden gemeten door het gebruik van subjectieve meetinstrumenten (vragenlijsten en dagboeken) of objectieve meetinstrumenten (dubbel gelabeld water of indirecte calorimetrie). De voor- en nadelen van deze meetinstrumenten worden verder besproken. Voortvloeiend uit de voor- en nadelen van deze meetinstrumenten wordt beschreven dat activiteitenmeters een goed alternatief zijn om te gebruiken in de zorg. Activiteitenmeters worden al veel gebruikt buiten de zorg, door actieve mensen om bijvoorbeeld stappen, hartslag en calorieën te meten. Voordelen van activiteitenmeters zijn dat ze objectief en continue meten en ook gemakkelijk in gebruik kunnen zijn. Er zijn echter nog weinig studies gedaan naar de validiteit en hanteerbaarheid van activiteitenmeters bij mensen met een langzame loopsnelheid en/of afwijkend looppatroon en verminderde fysieke activiteit zoals bij ouderen of mensen met een chronische aandoening. Het hoofdstuk sluit af met de constatering activiteitenmeters nog nauwelijks worden gebruikt in de zorg. Daarnaast zijn er ook nog geen handreikingen of richtlijnen voor zorgprofessionals hoe ze activiteitenmeters kunnen gebruiken in hun dagelijkse zorg. Vanuit deze constatering worden het doel, de vraagstelling en de structuur van dit proefschrift beschreven.

Hoofdstuk twee beschrijft een validatiestudie. Deze studie onderzocht de validiteit van negen (in 2016) commercieel verkrijgbare activiteitenmeters bij 130 patiënten met een chronische aandoening die fysiotherapie ontvangen. De geselecteerde activiteitenmeters waren de Accupedo (Corusen LLC), Activ8 (Remedy Distribution Ltd), Digi-Walker CW-700 (Yamax), Fitbit Flex (Fitbit inc), Lumoback (Lumo Bodytech), Moves (ProtoGeo Oy), Fitbit One (Fitbit inc), UP24 (Jawbone), and Walking Style X (Omron Healthcare Europe BV). De deelnemers droegen steeds minimaal twee activiteitenmeters terwijl ze een gestandaardiseerd activiteiten protocol met diverse algemene dagelijkse levensverrichtingen (ADL) uitvoerden. De activiteiten bestonden bijvoorbeeld uit stofzuigen of lopen met een boodschappentas. De uitvoering van het protocol is opgenomen met een videocamera en het aantal stappen op de video-opnames werd geteld door de onderzoekers (gouden standaard). Het aantal stappen gemeten met de activiteitenmeters werd vergeleken met het werkelijk aantal uitgevoerde stappen. De validiteit van de activiteitenmeters werd geanalyseerd door gebruik te maken van correlatiecoëfficiënten, t-testen, scatterplots en Bland-Altman plots. Het bleek dat bij alle activiteitenmeters de samenhang (correlatie) tussen het aantal stappen van de activiteitenmeters en het aantal stappen op de video opnames laag was (tussen de -0.02 en 0.33). Alle activiteitenmeters behalve de Fitbit One, hadden een significante systematische afwijking van de gouden standaard. Dat houdt in dat alle 
activiteitenmeters tijdens ADL activiteiten altijd anders (meer of minder) meten dat het werkelijk aantal stappen. De scatter- en Bland-Altman plots lieten een grote spreiding in scores zien met een gemiddelde onderschatting van het aantal stappen voor alle activiteitenmeters behalve de Activ8, deze had een gemiddelde overschatting van het aantal stappen. Deze studie laat zien dat de validiteit van negen commercieel verkrijgbare activiteitenmeters laag is bij het meten van stappen tijdens ADL activiteiten bij patiënten met een chronische aandoening die fysiotherapie ontvangen.

Hoofdstuk drie beschrijft de ervaringen van ouderen met een chronische aandoening die in behandeling waren bij een fysiotherapeut met het gebruik van commercieel verkrijgbare activiteitenmeters. De geselecteerde activiteitenmeters waren dezelfde als in hoofdstuk 2. Aan deze kwalitatieve studie deden negenentwintig ouderen met een chronische aandoening mee. Elke deelnemer droeg minstens één activiteitenmeter gedurende twee tot acht weken. In individuele interviews en een focusgroep interview is gevraagd naar hun ervaringen met de activiteitenmeters. Aan de hand van een framework werden de ervaringen geanalyseerd (deductief en inductief). Het framework was opgesteld, op basis van de literatuur en een expertpanel. Het framework bestond uit acht categorieën: aankoop, instructie, eigenschappen, correct functioneren, data delen, privacy, gebruik en interesse in feedback. De deelnemers gaven aan dat de activiteitenmeter(s) hen motiveerde om actiever te zijn, maar ervaarden het standaard stappendoel (10.000) als te hoog en de activiteitenmeter als te complex. Deelnemers gaven aan dat ze meer begeleiding van hun fysiotherapeut nodig hebben om de activiteitenmeters optimaal te kunnen gebruiken zowel tijdens de behandelingen als daarbuiten. Daarnaast ervaarden de deelnemers soms technische problemen met de activiteitenmeters. Ze waren bereid de data delen met de fysiotherapeut en om maximaal vijftig euro uit te geven aan een activiteitenmeter. Deze studie laat dus zien dat als activiteitenmeters ingebed worden in de fysiotherapie er meer aandacht moet zijn voor integratie van de activiteitenmeter in de behandeling, voor interpretatie van de data en voor het instellen van de activiteitenmeter voor en met de patiënt. Daarnaast gaf de studie inzicht in belangrijke concepten over hanteerbaarheid voor mensen met een chronische aandoening bij gebruik van activiteitenmeters.

Hoofdstuk vier presenteert de resultaten van een studie met twee doelen: 1) het (door)ontwikkelen van een userinterface van een bestaande activiteitenmeter (de MOX) samen met thuiswonende ouderen met of zonder chronische aandoening en 2) het inventariseren van het gebruik en de ervaringen van thuiswonende ouderen met of zonder chronische aandoening met de doorontwikkelde activiteitenmeter de Meten Is Super Simple (MISS) activity. Om de userinterface te ontwikkelen werd de double 
diamond methode gebruikt. De double diamond methode bestaat uit vier fases, eerst de 'discover' fase waarin men zich verdiept in de context van het probleem, daarna volgt de 'define' fase waarin de informatie die is opgedaan in de eerste fase bij elkaar wordt gebracht. Dit samen vormt de eerste 'diamand'. Vervolgens volgt de 'develop' fase waarin ontwerpen worden bedacht en ontwikkeld op basis van de opgedane informatie uit de eerste diamant. Als laatste volgt de 'deliver' fase waarin het ontwerp wordt getest en doorontwikkeld. Deze twee fases vormen de tweede 'diamand'. Als startpunt in de discover en define fase is een aantal algemene design-vereisten gebruikt die het gebruik van technologie voor ouderen faciliteren. Door middel van co-creatie met vijfendertig ouderen in de develop en deliver fase zijn het design, het feedback system en de app verder ontwikkeld naar een gebruiksvriendelijke userinterface voor deze doelgroep. Daarna zijn het gebruik en de ervaringen met de MISS Activity onderzocht. Hiervoor hebben achtentwintig ouderen de MISS Activity twee weken gebruikt. Hun ervaringen zijn verzameld door middel van individuele interviews. De deelnemers gaven aan dat de MISS Activity gemakkelijk te gebruiken was; ze hadden minimale hulp nodig bij het installeren en het gebruik. Deelnemers gaven daarnaast ook aan dat ze dachten dat in principe iedereen de MISS Activity zou kunnen gebruiken. De MISS Activity lijkt succesvol te zijn doorontwikkeld. Naast het ontwikkelen van een activiteitenmeter, kan deze studie ook een voorbeeld zijn hoe op een gestructureerde manier een userinterface van bestaande activiteitenmeters aangepast kan worden naar de vaardigheden en behoeften van een doelgroep.

Hoofdstuk vijf beschrijft een validatiestudie van het (door)ontwikkelde algoritme van de MISS activity (parameter optimalisatie voor draaglocatie, activiteiten en loopsnelheid). Dit algoritme ( MOX $\left._{\text {MissActivity }}\right)$ meet het aantal stappen en fysieke activiteit bij ouderen met of zonder chronische aandoening tijdens ADL activiteiten. Twintig ouderen hebben een activiteitenprotocol gebaseerd op ADL activiteiten (bijvoorbeeld stofzuigen) uitgevoerd. Het protocol werd gefilmd en het aantal stappen werd geteld (gouden standaard) en de tijd die dynamisch (lopend), staand en sedentair (zittend of liggend) werd doorgebracht werd gemeten. Om de resultaten beter te kunnen interpreteren zijn de data van de MOX $_{\text {Missactivity }}$ vergeleken met de resultaten van drie andere activiteitenmeters die verschillende onderliggende algoritmes gebruiken: de $\mathrm{MOX}_{\text {Annegran, }}$ de activPAL en de Fitbit Alta HR. De validiteit werd beoordeeld en in kaart gebracht door het fout percentage, het absolute fout percentage, Bland-Altman plots en correlatie coëfficiënten te berekenen. Voor het aantal stappen had de MOX $_{\text {Missactivity }}$ een gemiddelde absolute fout percentage van $9,3 \%$ en een correlatie coëfficiënt van 0,88 in vergelijking met de gouden standaard. De gemiddelde absolute fout percentage van dynamisch, staan en sedentaire tijd waren respectievelijk 15,9\%, 19,9\% en 9,6\%. De 
correlatiecoëfficiënten waren respectievelijk 0,55, 0,91 en 0,92 in vergelijking met de

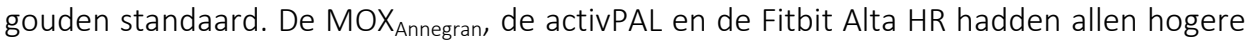
absolute fout percentages en lagere correlaties voor alle variabelen. De geoptimaliseerde parameter instellingen van het algoritme van de MISS activity kan het aantal stappen en fysieke activiteit beter (meer valide) meten gedurende ADL activiteiten bij ouderen met of zonder chronische aandoening in vergelijking met de drie vergelijkende algoritmes.

In hoofdstuk zes werd de MISS Activity geïntegreerd in de dagelijkse zorg. Dit onderzoek had twee doelen 1) het ondersteunen van zorgprofessionals en patiënten bij het implementeren van een activiteitenmeter in hun dagelijkse handelen, waarbij het klinisch redeneren klinisch redeneren van zorgprofessionals en de patiëntbetrokkenheid worden ondersteund, 2) kennis opdoen rondom het implementatie proces van activiteitenmeters in de zorg. Deze studie werd uitgevoerd in een gespecialiseerde GGZinstelling voor mensen met ernstige somatisch symptoomstoornis (wervelkolomgericht met pijn). Zorgprofessionals (psychosomatisch therapeuten) en de patiënten participeerden in een actieonderzoek. Dit actieonderzoek werd gebruikt zodat de psychosomatisch therapeuten en de patiënten tijd hadden om de activiteitenmeter uit te proberen in de dagelijkse zorg. Daarna konden ze hun ervaringen delen en op basis van deze ervaringen na gaan hoe en wanneer ze de activiteitenmeter het beste kunnen inzetten en dit vervolgens weer kunnen uitproberen en evalueren. Als startpunt is een concept handleiding ontwikkeld die de therapeuten gedurende het onderzoek op basis van hun ervaringen aangepast hebben. De data collectie is uitgevoerd door middel van geluidsopnamen van de gesprekken over fysieke activiteit in combinatie met de activiteitenmeter tijdens de therapiesessies, reflectiesessies met de therapeuten en semigestructureerde individuele interviews met de patiënten. De geluidsopnamen werden geanalyseerd door middel van een directed content analyse, gebaseerd op het framework van hoofdstuk drie. In totaal hebben drie therapeuten en elf patiënten deelgenomen. Achtentwintig gesprekken gedurende de therapiesessies, vier reflectie sessies en elf semigestructureerde interviews zijn opgenomen in drie iteratie cycli. Gedurende de iteraties hebben de therapeuten de handleiding verder aangepast en daarbij gebruik gemaakt van hun theoretisch denkkader (het Pijngevolgen model) en hun klinisch redeneren. In de handleiding en het bijbehorend stroomdiagram staat beschreven voor welke patiënten het gebruik van activiteitenmeters geschikt en minder geschikt is, welke er doelen er gesteld kunnen worden met een activiteitenmeter, hoe de doelen kunnen worden toegepast op de patiënt en hoe de data besproken kan worden. Om tot deze resultaten te komen en voldoende reflectie en diepgang te bereiken hadden de therapeuten aanzienlijke ondersteuning nodig van het 
onderzoeksteam. Door actieonderzoek als onderzoeksmethode te gebruiken hadden therapeuten de mogelijkheid om samen te leren, te reflecteren en om de activiteitenmeters daadwerkelijk te integreren in de dagelijkse zorg. Deze studie geeft enerzijds een voorbeeld hoe activiteitenmeters praktisch in de zorg kunnen worden ingezet en anderzijds als voorbeeld dienen hoe het implementatieproces van eHealth zou kunnen verlopen.

Hoofdstuk zeven, beschrijft de belangrijkste resultaten van de drie fasen van dit proefschrift. Daarna worden de methodologische overwegingen bediscussieerd. Ten eerste wordt de kracht van de structuur van de proefschrift en de onderzoekdesigns besproken. In fase één werd er onderzoek gedaan naar de klinimetrische eigenschappen van commercieel verkrijgbare activiteitenmeters. Gebaseerd op die resultaten is in fase twee een nieuwe activiteitenmeter ontwikkeld; de MISS Activity. Om de opgehaalde kennis terug te brengen naar de praktijk, is de MISS Activity geïmplementeerd in de zorg. Ten tweede wordt het selectieproces en de relevantie van de gebruikte activiteitenmeters bediscussieerd. De gebruikte activiteitenmeters zijn gekozen op basis van een aantal eigenschappen welke bepaald waren in overleg met fysiotherapeuten. Er zijn willekeurig negen activiteitenmeters gekozen die verschillende eigenschappen hadden (draaglocatie, type activiteitenmeter en gemeten variabelen). Hierdoor is er een representatieve steekproef genomen van de in 2015/2016 beschikbare activiteitenmeters. Ten derde wordt de mogelijke bias van de doelgroep besproken. Het zou mogelijk kunnen zijn dat de deelnemers in de studies meer geïnteresseerd waren in technologie en ook meer technische vaardigheden hadden ten opzichte van hun leeftijdsgenoten. $\mathrm{Er}$ is echter een toename in het gebruik van technologie te zien bij ouderen, waardoor de resultaten van de studies waarschijnlijk te generaliseren zijn voor een steeds groter wordende groep ouderen. Als vierde worden de kracht en de beperkingen van de gebruikte methodologie om activiteitenmeters te valideren besproken. In de validatie studies van dit proefschrift is zo veel mogelijk het dagelijks leven nagebootst én gebruikt gemaakt van de gouden standaard (video opnames). Er zijn echter nog geen eenduidig protocollen en analyses om activiteitenmeters te valideren, maar er zijn wel een aantal studies die een voorstel doen. De studies in dit proefschrift voldoen aan alle voorgestelde protocollen en analyses. Hierdoor wordt er bijgedragen aan een consequente rapportages over validiteitsstudies waardoor vergelijking tussen verschillende studies makkelijker wordt. Ten vijfde en laatste wordt het overkoepelende concept hanteerbaarheid besproken. Hanteerbaarheid is een brede term waardoor het zou kunnen dat niet alle aspecten ingebed zijn in het ontwikkelde framework in hoofdstuk drie. Dit framework is daarom een dynamisch framework en kan wellicht 
worden aangevuld met nieuwe inzichten uit nieuwe contexten zoals bijvoorbeeld een ziekenhuis.

Vervolgens zijn de geleerde lessen gedurende dit proefschrift beschreven, zoals het inzicht dat stakeholders een belangrijke rol hebben in succesvol onderzoek en dat er verschillende methoden zijn om deze stakeholders optimaal te betrekken. De MoSCoW methode, het businessmodel canvas, prototyping en de zes denkhoeden zijn hier voorbeelden van die in dit proefschrift zijn gebruikt. Daarna is beschreven dat het gebruik van activiteitenmeters geen compleet andere competenties vraagt dan die welke zorgprofessionals al hebben; zij beschikken al over verschillende vaardigheden om activiteitenmeters succesvol te kunnen gebruiken. Echter moeten ze nog extra eHealth competenties ontwikkelen om de volledige integratie te kunnen bewerkstellingen. Het laatste deel beschrijft de uitdagingen en noodzaak van implementatie van activiteitenmeters in de dagelijkse zorg en beschrijft verschillende methodes om deze implementatie te bereiken. Belangrijke aspecten om innovaties te implementeren zijn onder andere inzicht in factoren die het gebruik belemmeren en bevorderen en verschillende rondes (iteraties) om te leren de innovatie in de praktijk toe te passen.

Als laatste zijn de implicaties voor toekomstig onderzoek, onderwijs en de zorg beschreven. Onder andere is beschreven dat vervolgonderzoek zou kunnen focussen op het implementeren van activiteitenmeters in de zorg waarbij de stakeholders worden betrokken door middel van co-creatie methodieken en participatieve onderzoekdesigns. Daarnaast moet het onderwijs meer focussen op de benodigde eHealth competenties van studenten en professionals zodat zij zelfstandig eHealth kunnen gaan gebruiken in hun dagelijkse zorg. 



\section{Impact paragraph}

This chapter describes how the findings of this dissertation are relevant to society. First, the aim and main results are presented, followed by the relevance of this dissertation. Then, the relevance of the findings for stakeholders are reported. Finally, the dissemination activities are described.

\section{Aim and main results}

The main aim of this dissertation was to increase knowledge about the meaningful* use of activity trackers in healthcare and (older) adults with chronic diseases and for older adults with or without chronic diseases. This dissertation had three phases in which five studies took place. Phase one assessed the validity and feasibility of commercially available activity trackers. Both appeared to be insufficient for (older) adults with a chronic disease with a chronic disease. In phase two, an existing activity tracker was (re)designed for older adults with or without a chronic disease by adjusting the algorithm and upgrading the user interface. The new activity tracker was called the 'Measure It Super Simple (MISS)' Activity. Older adults evaluated the MISS Activity and found the tracker feasible and easy to use. The optimised algorithm was more valid during activities of daily living in older adults with or without a chronic disease compared to the gold standard and three selected commercially available activity trackers. In phase three, the MISS Activity was integrated into daily clinical practice. Several iterations were needed for healthcare professionals to use the MISS Activity in a way that supported their clinical reasoning and patient engagement. The activity tracker was used both as an assessment tool and an intervention tool.

\section{Relevance of this dissertation}

The current Dutch healthcare system faces challenges to keep healthcare sustainable. By 2040, the Netherlands are predicted to have 9.8 million people with a chronic disease there are expected to be 1.6 million people 80 years or older. ${ }^{1,2}$ To keep healthcare sustainable, eHealth, including activity trackers, could be beneficial. ${ }^{3,4}$ Recently, the number of commercially available activity trackers has grown explosively. Activity trackers provide objective insight into physical activity levels and can improve physical activity level if combined with regular exercise or lifestyle guidance. ${ }^{5-8}$ Healthcare professionals such as physiotherapists ${ }^{9}$, nurses $^{10}$, and nutritionists ${ }^{11}$ are trained to 
provide life-style guidance and require insight into a patient's physical activity level for their clinical reasoning. For example the new guideline for physiotherapist for patients with chronic obstructive pulmonary disease (COPD), recommends to us an activity tracker to indicate whether a patient is sufficient active and describes that physiotherapists should educate patients about the importance of sufficient physical activity in relation with a healthy lifestyle ${ }^{12}$. Studies show that activity trackers also

enhance a patient's self-management and self-efficacy regarding physical activity. ${ }^{13,14}$ Self-monitoring could lead to improved coping, realistic goal setting, and improved quality of life for patients. ${ }^{15}$ However, despite their advantages and potential, activity trackers are only sporadically used in healthcare. This dissertation contributes insight and tools to use activity trackers in daily clinical healthcare practice in a meaningful way.

This dissertation was part of the Brightlands Innovation Programme Limburg Meet $(\text { LIME) })^{17}$, a program that facilitates smarter measurement methods and more efficient data collection for better care and health. Within this programme, this project was part of the theme of 'personalised wearables' along with another doctoral project (the Psymate). Both projects focused on implementing health technology tools in daily clinical practice. Because these projects overlapped, a collaboration was established. The results of both projects will be used for further development of the LIME programme. Further, a network between researchers, entrepreneurs, civilians and educators developed within LIME. This allowed us to collaborate, share and gain knowledge with several different disciplines throughout the course of this dissertation and continues to do so in further research.

\section{Target population and other stakeholders}

The results of this dissertation are relevant to several stakeholders: (older) Adults with or without a chronic disease, healthcare professionals, educators and students, researchers, and technology manufacturers.

\section{(Older) Adults with or without a chronic disease}

The knowledge obtained from this dissertation can benefit (older) adults with or without a chronic disease. The MISS Activity was designed specifically with and for older adults with or without a chronic disease. Results indicate that the algorithm developed for the MISS Activity can more validly measure step count and physical activity during activities of daily living compared to the three selected commercially available activity trackers. Older adults with or without a chronic disease experienced the user interface of the MISS Activity as feasible and could use it without support from third parties (e.g., 
healthcare professionals). This creates opportunities to measure their physical activity level in a valid and feasible manner for (older) adults with or without a chronic disease. Although the MISS Activity is not available for purchase through websites or stores, it is available from the manufacturer. Healthcare professionals' meaningful use of an activity tracker in their daily clinical practice may improve the quality of care for (older) adults with or without a chronic disease.

\section{Healthcare professionals}

Although only physiotherapists and psychosomatic therapists participated in the studies within this dissertation, the findings are potentially relevant to several healthcare professionals such as occupational therapists, nurses, and nutritionists. For instance, the developed feasibility framework (found in the appendix to chapter three) can be used directly in daily clinical practice. Healthcare professionals also can benefit from the availability of the MISS Activity. By using a more valid activity tracker for the measurement of physical activity in (older) adults with or without a chronic disease, healthcare professionals obtain better insight into their physical activity level.

Furthermore, healthcare professionals can benefit from the easy user interface, since it takes little time to get familiar and explain the activity tracker their patients. Healthcare professionals can benefit from the lessons learned from our action research study, through the manual and course for healthcare professionals in which these lessons were integrated.

\section{Educators and students}

The findings of this dissertation can be used in courses for healthcare students and professionals that discuss the development and use of activity trackers and other digital health technology. Although measurement tools are incorporated into healthcare course curricula, the use of eHealth is typically addressed in a limited way or not at all.16 As the use of eHealth is a method to ensure a sustainable healthcare system, one might advocate that the application of eHealth in daily clinical practice should be more addressed. Competencies in eHealth are a prerequisite for healthcare professionals to work with all sorts of health technology. In order to incorporate more eHealth into the curricula, lecturers should receive training focused on the opportunities and use of eHealth in healthcare. For example, Zuyd University of Applied Sciences pilots a program to help educators make decisions on implementing eHealth, with specific attention to eHealth measurement technologies into the curricula of seven health educations (physiotherapy, occupational therapy, speech therapy, nursing, midwifery, social work 
and arts therapies) and to gain insight into the support needs of educators during the implementation.

The developed manual can be used as an example of how to integrate activity trackers and other health technologies and to teach students how to use these tools in a meaningful way. Students can benefit from the feasibility framework and the important variables listed when choosing and/or developing an activity tracker for (older) adults with or without a chronic disease.

\section{Researchers}

This dissertation is a first step toward integrating the use of activity trackers in healthcare. Researchers can use the findings of this dissertation regarding the validity, feasibility, and implementation of activity trackers in (older) adults with or without a chronic disease. . The developed feasibility framework also can be used when choosing an activity tracker to incoporate in their research or to test the feasibility and use of other activity trackers and other possible eHealth tools. The MISS Activity can be used in future research; in two upcoming studies the MISS Activity will be used as a measurement tool for physical activity in older adults and patients with COPD. Additionally, researchers could learn from the phases of the studies performed in this dissertation and the designs and methods (e.g., action research and co-creation methods). For future research focusing on the use of activity trackers in other healthcare settings, the design and results of our action research study (chapter 6) might be a suitable starting point. Researchers also could study the effect of activity trackers on physical activity in healthcare in (older) adults with or without a chronic disease.

\section{Technology manufacturers}

The MISS Activity is available for purchase via the manufacturer for both individual users and researchers or institutes (e.g., hospitals, research centres). Technology manufacturers also might benefit from the developed feasibility framework and the derived list of (older) adults with or without a chronic disease when choosing an activity tracker. Furthermore, this dissertation might encourage technology manufacturers and healthcare professionals to collaborate more frequently when developing new eHealth tools.

Table 1 describes the dissemination activities performed during this dissertation. 
Table 1. Knowledge transfer to healthcare, research, and education.

\begin{tabular}{|c|c|}
\hline \multicolumn{2}{|c|}{ Knowledge transfer to healthcare, research, and education } \\
\hline Presentations & 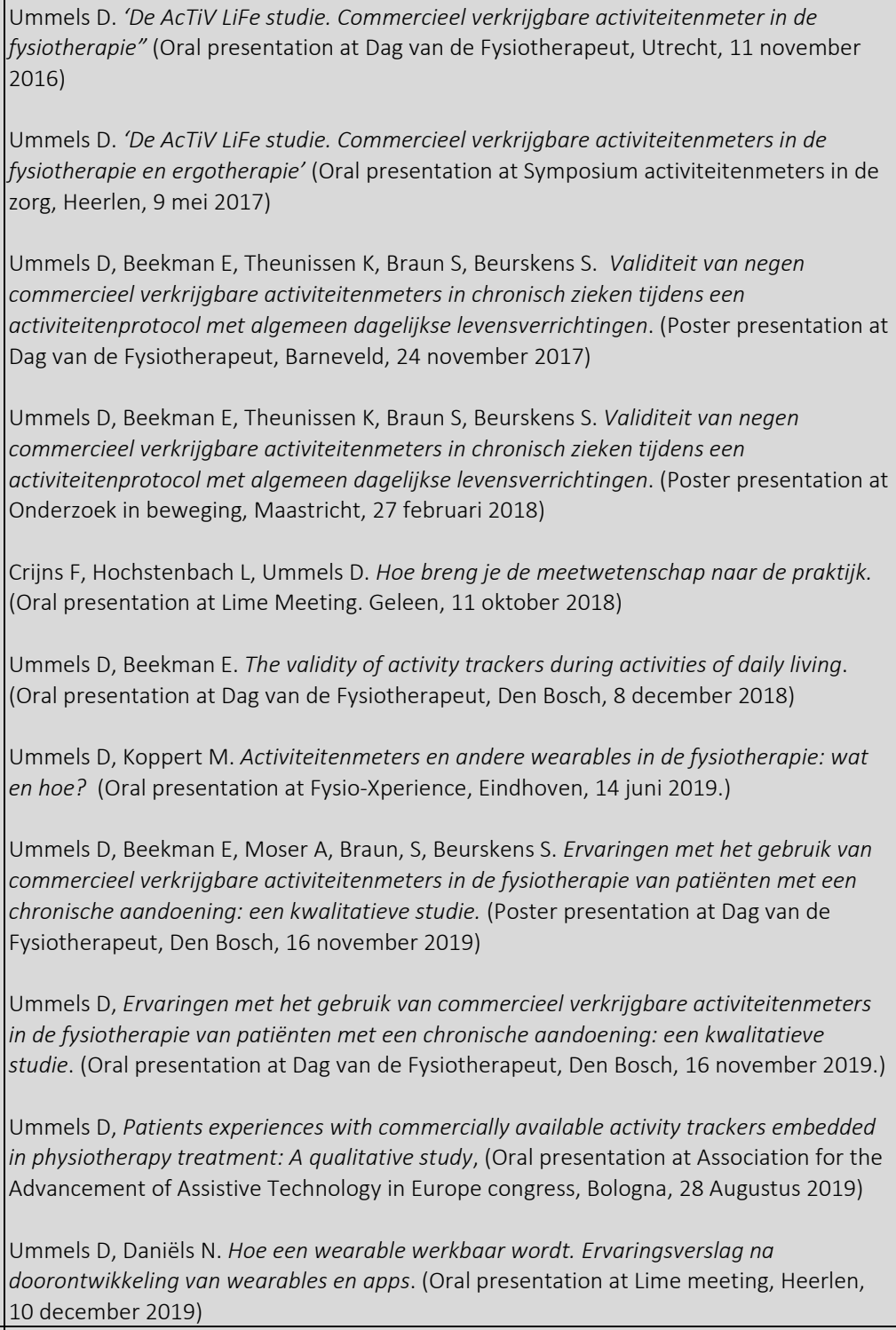 \\
\hline $\begin{array}{l}\text { Publications in } \\
\text { national } \\
\text { professional } \\
\text { journals }\end{array}$ & $\begin{array}{l}\text { Ummels D., Beekman E., Braun S., Theunissen K., Moser A., Beurskens S. Validiteit en } \\
\text { ervaringen in de dagelijkse praktijk. Commercieel verkrijgbare activiteitenmeters. } \\
\text { Fysiopraxis. 2019. Jaargang } 28 \text { december 2019/januari } 2020 . \\
\text { Ummels D. Beekman E., Moser A., Braun S., Beurskens A. Ervaring van patiënten met een } \\
\text { chronische aandoening met het gebruik van commercieel verkrijgbare activiteitenmeters } \\
\text { in de fysiotherapie. Physios. } 2020 \text { Jaargang 12. Nummer } 1 .\end{array}$ \\
\hline
\end{tabular}




\begin{tabular}{|c|c|}
\hline Multimedia & $\begin{array}{l}\text { Several online publications in layman's terms about the studies of this dissertation. } \\
\text { Zuyd University of Applied Sciences: } \\
\text { https://www.zuyd.nl/onderzoek/lectoraten/projecten-autonomie-en- } \\
\text { participatie/promotie/activiteitenmeters-in-de-gezondheidszorg } \\
\text { https://www.zuyd.nl/over-zuyd/nieuws/2020/11/lime } \\
\text { Limburg Meet: } \\
\text { https://www.limeconnect.nl/nieuws/meten-bij-ouderen/ } \\
\text { https://www.limeconnect.nl/nieuws/commercieel-verkrijgbare-activiteitenmeters/ } \\
\text { https://www.limeconnect.nl/nieuws/commercieel-verkrijgbare-activiteitenmeters-vaak- } \\
\text { ingewikkeld/ } \\
\text { https://www.limeconnect.nl/nieuws/commerciele-fitness-trackers-schieten-te-kort/ } \\
\text { Smart Health: } \\
\text { https://www.smarthealth.nl/2018/04/19/onderzoek-commerciele-fitness-trackers- } \\
\text { schieten-te-kort-voor-patienten-met-chronische-aandoening/ } \\
\text { https://www.smarthealth.nl/2019/04/30/fysiotherapeuten-en-hun-patienten-nog-niet- } \\
\text { gewend-aan-inzet-stappenteller-of-app/ } \\
\text { FMT Gezondheidszorg: } \\
\text { https://fmtgezondheidszorg.nl/commercieel-verkrijgbare-activiteitenmeters-vaak-te- } \\
\text { ingewikkeld-voor-mensen-met-een-chronische-ziekte/ } \\
\text { Nieuws voor leefstijlcoaches: } \\
\text { https://www.nieuwsvoordietisten.nl/activiteitenmeters-zijn-vaak-te-ingewikkeld/ } \\
\text { ICT\& Health: } \\
\text { https://www.icthealth.nl/nieuws/wearable-vaak-nog-te-ingewikkeld-voor-gebruiker/ } \\
\text { https://www.accelerometry.eu/consumer-activity-monitors-often-too-complicated-for- } \\
\text { people-with-chronic-diseases/ } \\
\text { https://www.accelerometry.eu/miss-activity-valid-and-user-friendly-measuring/ } \\
\text { https://www.accelerometry.eu/commercial-acitvity-trackers-fall-short-for-patients-with- } \\
\text { chronic-illness/ }\end{array}$ \\
\hline Video clip & $\begin{array}{l}\text { One video about the use of activity trackers in physiotherapy has been developed } \\
\text { belonging to the publication in Physios. } \\
\text { https://www.physios.nl/tijdschrift/editie/artikel/t/ervaringen-van-patienten-met-een- } \\
\text { chronische-aandoening-met-het-gebruik-van-commercieel-verkrijgbare- } \\
\text { activiteitenmeters-in-de-fysiotherapie }\end{array}$ \\
\hline Healthcare course & $\begin{array}{l}\text { Course for healthcare professionals on how to use activity trackers in daily clinical } \\
\text { practice taught at Zuyd University of Applied Sciences. }\end{array}$ \\
\hline Manual & $\begin{array}{l}\text { Manual 'how to use activity trackers in daily clinical practice' which can be used in } \\
\text { healthcare, education, and research }\end{array}$ \\
\hline Framework & $\begin{array}{l}\text { Development of the feasibility framework which can be used in healthcare, education, } \\
\text { and research (appendix of chapter three). }\end{array}$ \\
\hline Symposium & $\begin{array}{l}\text { Organised Symposium activiteitenmeters in de zorg (Zuyd Hogeschool, Heerlen, } 9 \text { mei } \\
\text { 2017) }\end{array}$ \\
\hline Lectures & $\begin{array}{l}\text { Commercieel verkrijgbare activiteitenmeter in de fysiotherapie en ergotherapie. Zuyd } \\
\text { University of applied science, bachelor track physiotherapy. } 2016 \text {. }\end{array}$ \\
\hline
\end{tabular}




\begin{tabular}{|c|c|}
\hline $\begin{array}{l}\text { Inclusion of } \\
\text { students }\end{array}$ & $\begin{array}{l}129 \text { students from different disciplines of Zuyd University of Applied Sciences: } \\
\text { physiotherapy, communication and multimedia design, ICT, health } \\
\text { technology/healthcare biometrics, facility management, pre-university education, and } \\
\text { international business. (https://www.zuyd.nl/over-zuyd/nieuws/2020/11/lime) }\end{array}$ \\
\hline Publications & $\begin{array}{l}\text { All articles in this dissertation are published in international peer-reviewed journals. } \\
\text { Counting Steps in Activities of Daily Living in People With a Chronic Disease Using Nine } \\
\text { Commercially Available Fitness Trackers: Cross-Sectional Validity Study. Ummels D, } \\
\text { Beekman E, Theunissen K, Braun S, Beurskens A. JMIR Mhealth Uhealth, 2018; 2(6);e70 } \\
\text { Patients' experiences with commercially available activity trackers embedded in } \\
\text { physiotherapy treatment: a qualitative study. Ummels D, Beekman E, Moser A, Braun S, } \\
\text { Beurskens A. Disability and Rehabilitation, 2020; 42(23), 3284-3292 } \\
\text { Measure It Super Simple (MISS) activity tracker: (re)design of a user-friendly interface } \\
\text { and evaluation of experiences in daily life. Ummels D, Braun S, Stevens A, Beekman E, } \\
\text { Beurskens A. Disability and Rehabilitation: Assistive technology, 2020; 24;1-11 online } \\
\text { ahead of print. } \\
\text { The Validation of a Pocket Worn Activity Tracker for Step Count and Physical Behavior in } \\
\text { Older Adults during Simulated Activities of Daily Living. } \\
\text { Ummels D*, Bijnens W*, Aarts J, Meijer K, Beurskens A, Beekman E. Gerontology and } \\
\text { Geriatric medicine, 2020; 30-6;2333721420951732. } \\
\text { Using an Activity Tracker in Healthcare: Experiences of Healthcare Professionals and } \\
\text { Patients. Ummels D., Beeman E., Braun S., Beurskens A. International Journal of } \\
\text { Environmental Research and Public Health. 2021, 18(10), 5147 } \\
\text { One article describing the study protocol of phase one is published in a international } \\
\text { peer-reviewed journal. } \\
\text { Beekman E, Braun S, Ummels D, van Vijven K, Moser A, Beurskens A. Validity, reliability } \\
\text { and feasibility of commercially available activity trackers in physical therapy for people } \\
\text { with a chronic disease: a study protocol of a mixed methods research. Pilot Feasibility } \\
\text { Stud. 2017; Nov 23;3:64. }\end{array}$ \\
\hline Follow-up grants & $\begin{array}{l}\text { One follow-up grant has been granted: SIA KIEM-HBO. De verkenning van de } \\
\text { ontwikkeling en evaluatie van een stappenplan voor geintegreerd gebruik van } \\
\text { activiteitenmeters binnen de zorg voor mensen met chronische pijn. } \\
\text { One follow-up grant has been submitted: Meten op maat met eHealth, Methodisch } \\
\text { ondersteunen bij het duurzaam gebruik in de eerstelijns zorgpraktijk. }\end{array}$ \\
\hline Spin-off projects & $\begin{array}{l}\text { One internal Zuyd University project within the transitation theme 'Gezonde } \\
\text { Samenleving' where educators are supported in make decisions on implementing } \\
\text { eHealth, with specific attention to eHealth measurement technologies into the curricula } \\
\text { of seven health educations (physiotherapy, occupational therapy, speech therapy, } \\
\text { nursing, midwifery, social work and arts therapies) and to gain insight into the support } \\
\text { needs of educators during the implementation. } \\
\text { The MISS Activity can be used in future research, two studies are already planning on } \\
\text { using the MISS Activity as a measurement tool for physical activity in older adults } \\
\text { (project: H2O2O Pharaon) and patients with COPD (project: COPD subtypes based on } \\
\text { Western and Chinese diagnostics). }\end{array}$ \\
\hline
\end{tabular}




\section{References}

1. Menting J, van Schelven F, Grosscurt R, Spreeuwenberg P, Heijmans M. Zorgmonitor 2019 Ontwikkelingen in de zorg voor mensen met een chronische ziekte: 2005-2018. $1^{\text {ste }}$ Edition. Utrecht: NIVEL; 2019.

2. Centraal Bureau voor Statistiek. Prognose: 19 miljoen inwoners in 2039. URL: https://www.cbs.nl/nl$\mathrm{nl} /$ nieuws/2019/51/prognose-19-miljoen-inwoners-in-2039. [accessed 2021-01-30].

3. Hoy MB. Personal Activity Trackers and the Quantified Self. Med Ref Serv Q 2016; 35(1): 94-100. PMID: 26794199.

4. Bassett DR, Freedson PS, John D. Wearable Activity Trackers in Clinical Research and Practice. Kinesiology Review 2019; 8(1): 11-5.

5. Funk M, Taylor EL. Pedometer-based walking interventions for free-living adults with type 2 diabetes: a systematic review. Current diabetes reviews 2013; 9(6): 462-71. PMID: 24160358.

6. Kawagoshi A, Kiyokawa N, Sugawara K, et al. Effects of low-intensity exercise and home-based pulmonary rehabilitation with pedometer feedback on physical activity in elderly patients with chronic obstructive pulmonary disease. Respiratory medicine 2015; 109(3): 364-71. PMID: 25682543.

7. Mendoza L, Horta P, Espinoza J, et al. Pedometers to enhance physical activity in COPD: a randomised controlled trial. The European respiratory journal 2015; 45(2): 347-54. PMID: 25261324.

8. Kononova A, Li L, Kamp K, et al. The Use of Wearable Activity Trackers Among Older Adults: Focus Group Study of Tracker Perceptions, Motivators, and Barriers in the Maintenance Stage of Behavior Change. JMIR Mhealth Uhealth 2019; 7(4): e9832. PMID: 30950807.

9. Crajé MC, Hodselmans AP, van Ittersum MW, van Heeringen-de Groot D, Verhoef J, van der Schans CP. Introduction Royal Dutch Society for Physical Therapy physical activity intervention. URL: https://www.fysionet-evidencebased.nl/images/pdfs/beweeginterventies/inleiding_bij_de_kngfstandaarden_beweeginterventies.pdf (accessed 2021-01-30).

10. Schuurmans M, Lambregts J, Grotendorst A. Beroepsprofiel verpleegkundige. URL: http://www.invoorzorg.nl/docs/ivz/informatiecentrum/professionals/23203\%20Beroepsprofiel\%20verple egkundige.pdf (accessed 2021-01-30).

11. Nederlandse Vereniging van Dietisten. Beroepsprofiel diëtist 2013. URL: https://www.nvdietist.nl/ images/Wetgeving/Beroepsprofiel_2013.pdf (accessed 2021-01-30).

12. Vreecken, HL, Beekman E, van Doornmaal MCM, Post MHT, Meerhoff GA, Spruit MA. KNGF Richtlijn COPD. URL: https://www.kngf2.nl/binaries/content/documents/kngf-kennisplatform/producten/ richtlijnen/copd-2020/copd-2020/kngfextranet\%3Adownload (accessed 2021-02-02).

13. Chiauzzi E, Rodarte C, DasMahapatra P. Patient-centered activity monitoring in the self-management of chronic health conditions. BMC medicine 2015; 13: 77. PMID: 25889598.

14. Karapanos E, Gouveia R, Hassenzahl M, Forlizzi J. Wellbeing in the Making: Peoples' Experiences with Wearable Activity Trackers. Psychology of well-being 2016; 6: 4. PMID: 27376017.

15. Richard AA, Shea K. Delineation of self-care and associated concepts. J Nurs Scholarsh 2011; 43(3): 25564. PMID: 21884371.

16. Wentink MM, Siemonsma PC, van Bodegom-Vos L et al. Teachers' and students' perceptions on barriers and facilitators for eHealth education in the curriculum of functional exercise and physical therapy: a focus groups study. BMC Med Educ.2019 Sep 6;19(1):343. PMID: 31492129.

17. Brightlands Innovation Programme Limburg Meet. URL:https://www.limeconnect.nl/de-themas/ \#!/thema3 (accessed 2021-30-01). 


\section{Dankwoord}

Wie had gedacht, dat ik tijdens mijn masterscriptie ook al problemen zou ervaren bij het gebruiken van activiteitenmeters in de zorg? Verpleegkundigen die een activiteitenmeter weggooiden, patiënten die de meter niet fijn vonden zitten en meters waarvan je na een week dragen erachter komt dat ze niks hebben gemeten! Wat ik toen nog niet wist is dat deze problemen mij de komende vier jaren bezig zouden houden.

Als je gaat promoveren weet je, ondanks alle goed bedoelde adviezen, niet wat je te wachten staat en zonder een aantal mensen was ik nooit zo ver gekomen.

Als eerste mijn promotieteam: Sandra, Susy en Emmylou. Zonder alle sturing, feedback en motiverende woorden van jullie had dit proefschrift er niet nu niet gelegen. De discussies tijdens de promotie overleggen hebben mij altijd uitgedaagd om het meeste uit mijzelf te halen, zodat ik uit mijn comfortzone stapte. Jullie hebben mij alle drie gedurende dit promotietraject laten groeien als persoon.

Sandra, bedankt voor de eindeloze energie en passie die je hebt voor het praktijkgericht onderzoek. Je maakte altijd tijd om feedback te geven, om mijn vragen te beantwoorden of even te overleggen als ik ergens niet zeker over was. Op momenten dat ik overweldigd was door de hoeveelheid werk, de nieuwe informatie of, wanneer mijn hoofd helemaal vol zat na een promotie overleg wist je alles te relativeren. Dat heeft mij heel veel rust gegeven om te kunnen focussen op de zaken die echt belangrijk waren.

Susy, bedankt voor alle opbeurende en motiverende woorden. De kleine succesjes werden benoemd en geprezen, wat mij weer de energie gaf om door te gaan. Ongelooflijk hoe snel jij feedback en je ideeën op stukken gaf en mij uiteindelijk (soms moeilijke) keuzes liet maken zodat ik als onderzoeker kon groeien. Door het 'out of the box' denken heb ik veel nieuwe ideeën gekregen, nieuwe mensen ontmoet en met disciplines kennis gemaakt die allemaal ook een bijdragen hebben geleverd aan dit proefschrift.

Emmylou, tijdens mijn bachelor scriptie, waarin ik een heel ander onderzoek deed, was jij al mijn afstudeerbegeleider. Ik ben blij dat ik de afgelopen vier jaar jou weer als 'afstudeerbegeleider' mocht hebben. Je had altijd nieuwe ideeën en oplossingen op het moment dat het even niet liep zoals wij zouden willen. Je zag foutjes die anderen niet zagen en gaf feedback tot op de spatie, punt en komma. Mede door die kritische blik is dit proefschrift geworden wat het nu is. 
Leden van de beoordelingscommissie, Prof. Dr. Rik Crutzen, Prof. Dr. Ton Lenssen, Prof. Dr. Catherine Bolman, Dr. Ir. Marike Hettinga en Dr. Brenda Berendsen hartelijk dank voor het lezen en beoordelen van mijn proefschrift.

Daarna wil ik alle deelnemers van de onderzoeken in dit proefschrift bedanken, zonder hen had ik dit proefschrift nooit kunnen schrijven.

Ook wil ik alle paramedici en medewerkers die hebben meegewerkt van de deelnemende instellingen bedanken; ParaMedisch Centrum Zuid, Adelante Zorggroep, Ergotherapiepraktijk Zuid-Limburg, Fysiotherapie Geraets, Blokland \& Vrouenraets, Ziekenhuis Nij Smellinghe, Fontys Hogeschool, Hanzehogeschool Groningen, KNGF, Ergotherapie Nederland, het Rughuis en Yunify.

Maastricht Sport en Buurtnetwerk Wittevrouweveld, bedankt dat ik bij jullie langs mocht komen om deelnemers te werven en te vertellen over mijn onderzoek.

Een woord van dank voor Burgerkracht Limburg en in het speciaal Henk Marell en Harry Bakkels. Bedankt voor de waardevolle input die jullie hebben geleverd door mee te denken vanuit het patiënten perspectief.

Het 'MISS Activity groepje' kan natuurlijk niet ontbreken. Jos, bedankt dat je mij mee hebt genomen in de wereld van het ontwikkelen van wearables. An, zonder jouw kritische vragen en oplossingen was de MISS Activity niet geworden wat het nu is. Freek, bedankt dat je ons ook de commerciële kant van activiteitenmeters hebt laten zien. Dennis, bedankt voor het eindeloos aanpassen van de app, iedere keer wanneer ik hier om vroeg. Jolien, ik ben blij dat we zo'n creatief iemand in het team hadden; zonder jou had de app en de meter er nooit zo mooi uitgezien. Kenneth, bedankt voor het meedenken bij de onderzoekdesigns en de feedback op de geschreven artikelen. Wouter, door jou begrijp ik nu meer van algoritmes en wat zich afspeelt in die 'black box'. Bedankt voor de fijne samenwerking!

Een hoop studenten hebben deelgenomen aan dit proefschrift: Joey Gerritsen, Nursize Bilen, Wesley Maurmair, Jordi Hutjens, Renee Schroijen, Jorrit Geerlings, Job Hollands, Cederic Houben, Robin Jongen, Daniël Wauben, Loek Titulaer, Madeleine van Es, Svenja van Hove, Joëll Delaure, Lizzy Deneer, Lara Hermans, Laura Frissen, Erik Sauvé, Frank Helgers, Annika Borrmann, Aniek Kusters, Justin Fisscher, Jessey Schins en alle eerstejaars studenten (leerjaar 2017) Communication and Multimedia Design. 
Gaston Jamin, bedankt voor de fantastische begeleiding van al die eerstejaars CMD studenten én voor het maken van een aantal mooie plaatjes in een van mijn artikelen.

Collega's van het lectoraat autonomie en participatie: Marsha, Esther, Stephanie vH., Ruth, Eveline, Jolanda, Stephanie C. , Asiong, Sarah, Albine, Linda, Barbara, Hester, Steffy, Anita en Joyce bedankt dat jullie altijd interesse hadden in mijn onderzoek. We hebben een team met veel verschillende expertises waardoor er altijd wel iemand was die een antwoord op mijn vragen had.

Albine, Bedankt voor alle ondersteuning in mijn vragen over kwalitatief onderzoek. Ik heb heel veel van jou kunnen leren.

Laura, bedankt voor je ondersteuning en samenwerking bij de data-analyse van het laatste artikel.

Een speciaal woord van dank aan Stephanie, Prisca en Joyce, de talloze vragen die ik had hebben jullie telkens met evenveel geduld beantwoord. Er was altijd ruimte voor een praatje en ik kwam dan ook regelmatig langs, al was het maar voor de dropjes!

Dan de promovendi kamer: Jerome, Kyra, Li-Juan, Linda, Steffy, Stephie, Jolanda, Ruth, Renee en Hester. Helaas bestaat onze kamer niet meer, maar bedankt voor de leuke jaren die we daar hadden. Dit was de plek waar 'domme' vragen niet bestonden en eens goed te kunnen lachen om de perikelen die onderzoek doen met zich mee brengt. Speciaal woord van dank aan Kyra, die mij fantastisch geholpen heeft bij de eerste twee artikelen.

Dan al mijn collega's van de academie fysiotherapie, bedankt voor alle interesse in mijn proefschrift. Alle bemoedigende woorden deden mij deugd! Monique en Claudy, bedankt voor de kans die ik kreeg om ook mee te draaien in het onderwijs. Ik ben erg blij dat ik deze taken kan voorzetten en mij zelf hier verder in kan ontwikkelen. Speciaal woord van dank voor Yolande, waar ik mocht starten als afstudeerbegeleider bij de academie fysiotherapie.

Lieve PMC Collega's, ook jullie moet ik van harte bedanken. Ik ben heel blij dat ik nog een dag in de week als fysiotherapeut bij jullie mag werken. Het enthousiasme en de passie voor het vak leeft echt in deze praktijk wat maakt dat ik er ook erg graag werk. Met name Elke, Maartje, Mariëlle en Inez wil ik nog extra bedanken. Het is superfijn om jullie als directe collega's te hebben. De keren dat ik voor mijn onderzoek weer moest 
schuiven met patiënten en weer een beroep op jullie moest doen zijn ondertussen niet meer te tellen.

De nodige ontspanning tijdens het schrijven van mijn proefschrift vond ik met name in het sporten. En dit zou een stuk minder leuk zijn zonder mijn sportbuurtjes: Caren, llona en Natascha! Dank jullie wel daarvoor. Robin en Loes, bedankt voor jullie enthousiasme en leuke sportlessen!

Dewi, Pim, Robrecht, Paola, Stella, Leon, Zoë, Mathijs, Bas, Amelie, Maud, William, Bas, Lisa, Beaudille en Jessy, bedankt voor alle oprechte interesse in mijn proefschrift. Maar vooral bedankt voor alle afleiding die jullie boden, ik heb genoten van alle uitjes, feestjes en borrels de afgelopen jaren.

Ruth, lief en leed over het leed dat promoveren heet hebben wij de afgelopen jaren gedeeld. Urenlange telefoongesprekken zijn er gepleegd. Soms om te klagen en soms om de successen te vieren. Wij houden elkaar goed in balans: hard werken, maar ook genoeg ontspannen. Dit ging ook gepaard met genoeg speciaal biertjes, hapjes en barbecues. Ook Steven moet ik bedanken, want die heeft telkens maar het onderwerp 'promoveren' geduldig aangehoord. Ruth, ik vind het dan ook fanatisch dat jij mijn paranimf wilt zijn en kan niet wachten totdat jij mag gaan promoveren!

Dan mijn lieve 'middelbare school vriendinnetjes': Lonneke, Danique, Milou, Fabiënne en Emma. Wij kennen elkaar ondertussen al sinds de brugklas. Bedankt voor alle leuke uitjes, weekendjes weg, verjaardagen en sinterkerstennieuw avondjes. Met jullie duurt het nooit lang voordat een van ons de slappe lach heeft, maar is er ook ruimte voor een luisterend oor. Ik hoop dat wij nog heel lang onvergetelijke avonturen zullen meemaken.

Nympha, Henk, Paul, Isolde, Aliene, Huub, Chris, Jeffrey en Rick. Bedankt voor alle steun de afgelopen jaren en de eindeloze vragen hoe het met mijn onderzoek gaat. Al die interesse werd echt enorm gewaardeerd, en ik voel me dan ook helemaal thuis bij jullie!

Kirsten, Mike, Liam, Eloy, Maurice, Liliane, Youri en Mauro, bedankt voor alle steun en de afleiding door de familiefeestjes!

Oma Martha, Opa Jo, Oma Gerry en Opa Rein*, bedankt voor de interesse in mijn 'scriptie'. Maar ook voor alles wat jullie al mijn hele leven voor mij hebben gedaan. Hopelijk kunnen we nog lang van alles samen ondernemen. Oma Gerry, zoals jij altijd zegt: "Opa Rein kijkt vanaf zijn sterretje mee!" 
Kyra, Kiwi, sommige mensen zeggen dat wij heel veel op elkaar lijken, anderen zeggen juist van niet. Ik weet in ieder geval dat we elkaar goed aanvullen. Je hebt heel wat verhalen en discussies thuis moeten aanhoren over 'promoveren' en 'onderzoek doen'. Dat is misschien niet helemaal jouw ding, maar ik ben super blij dat je mijn paranimf wilt zijn! Alles wat bij het paranimf zijn hoort kan ik volledig aan je overlaten, want als je iets doet waar je goed in bent is het tot in de puntjes geregeld. Ga zo door waar je mee bezig bent, want je hebt heel veel in je mars.

Papa en Mama, door alles wat jullie mij geleerd en meegegeven hebben, ben ik gekomen waar ik nu ben. Jullie hebben mij altijd mijn eigen weg laten kiezen en mij daarin gesteund. Uiteindelijk is het een mix geworden tussen het onderzoek (mama) en fysiotherapie (papa). Mede daardoor hebben jullie mij heel veel kunnen helpen met de zaken waar ik tegen aan liep tijdens het onderzoek doen. Lieve papa en mama, bedankt voor alles!

Lieve Mike, jij hebt mijn promoveren van het meest dichtbij meegemaakt. Maar juist jij zorgde ervoor dat ik ook vaak genoeg afleiding had. Soms was het leuke afleiding zoals de vele reizen en uitstapjes die we maakten en soms was het afleiding in de vorm van 10 $\mathrm{kg}$ tomaten uit de moestuin waar ik saus van moest maken. Toen dit proefschrift eindelijk af was, waren we allebei blij dat we meer tijd voor elkaar kregen. Echter besloot jij dat je het ook een goed idee vond om te gaan promoveren. De rollen worden nu dus omgedraaid, maar dan heb ik over vier jaar wel een 'echte' grottendokter in huis. In de tussentijd ontferm ik me met alle liefde over je kilo's groenten uit de moestuin en ga ik mijn best doen jou net zo goed te ondersteunen als dat jij dat voor mij hebt gedaan! 



\section{About the author}

Darcy Ummels was born on August 28, 1994 in Ulestraten, the Netherlands. In 2011 she completed secondary school at Stella Maris College Meerssen. She studied physiotherapy at Zuyd University of Applied Sciences and graduated in 2015. Thereafter, she studied Human Movement Science at Maastricht University. Darcy graduated as a Master of Science in 2016 with a thesis about the effect of muscle strength and aerobic capacity on post-operative physical activity during hospitalisation in oncology patients.

In 2016 Darcy started working at ParaMedisch Centrum Zuid as physiotherapist. Alongside with her work as physiotherapists she started her PhD project at Zuyd

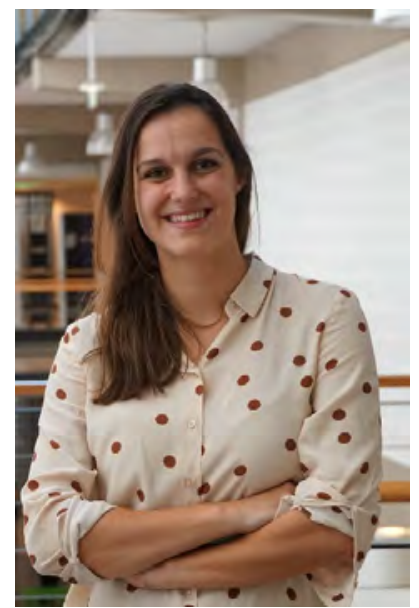

Photo by Franc Coenen University of Applied Sciences in 2016 that resulted in this dissertation. This was a collaborative project of the Research Centre for Autonomy and Participation of Persons with a Chronic Illness (Zuyd University), the Department of Family Medicine (Maastricht University) and CAPHRI School (Maastricht University).

This dissertation was part of the Brightlands Innovation Programme Limburg Meet (LIME), a programme that facilitates the development of smarter measurement methods and more efficient data collection for better care and health.

Currently, her work involves a combination of her work as physiotherapist at ParaMedisch Centrum Zuid, research at the Research Centre for Autonomy and Participation of Persons with a Chronic Illness and lecturing for the academy of physiotherapy of Zuyd University of Applied Sciences. The focus of her work is on the dissemination of the findings of this dissertation. The topic she is interested in include activity trackers, eHealth, implementation of eHealth and measuring in daily clinical practice. 



\section{List of publications}

\section{International peer-reviewed publications}

Ummels D., Beeman E., Braun S., Beurskens A. Using an Activity Tracker in Healthcare: Experiences of Healthcare Professionals and Patients. International Journal of Environmental Research and Public Health. 2021, 18(10), 5147

Ummels D., Braun S., Stevens A., Beekman E., Beurskens A. Measure It Super Simple (MISS) activity tracker: (re)design of a user-friendly interface and evaluation of experiences in daily life. Disability and Rehabilitation: Assistive Technology. 2020. Sep 24;1-11. Online ahead of print.

Ummels D., Bijnens W., Aarts J., Meijer K, Beurskens A, Beekman E. The validation of a pocket worn activity tracker for step count and physical behaviour in older adults during simulated activities of daily living. Gerontology and Geriatric Medicine. Sep 30;6:2333721420951732.

Bijnens W., Aarts J., Stevens A., Ummels D., Meijer K. Optimization and Validation of an Adjustable Activity Classification Algorithm for Assessment of Physical Behavior in Elderly. Sensors. 2019;19;5344.

Ummels D., Beekman E., Moser A., Braun S., Beurskens A. Patients' Experiences with Commercially Available Activity Trackers embedded in Physiotherapy Treatment: A Qualitative Study. Disability and Rehabilitation. 2020 Nov;42(23):3284-3292.

Ummels D., Beekman E., Theunissen K., Braun S., Beurskens A.. Counting Steps in Activities of Daily Living in People With a Chronic Disease Using Nine Commercially Available Fitness Trackers: Cross-Sectional Validity Study. JMIR Mhealth Uhealth 2018;6(4):e70.

Beekman E, Braun S.M., Ummels D., van Vijven K., Moser A., Beurskens A.J. Validity, reliability and feasibility of commercially available activity trackers in physical therapy for people with a chronic disease: a study protocol of a mixed methods research. Pilot and Feasibility Studies. 2017;23(3):e64. 


\section{National publications}

Ummels D., Beekman E., Braun S., Theunissen K., Moser A., Beurskens S. Validiteit en ervaringen in de dagelijkse praktijk. Commercieel verkrijgbare activiteitenmeters. Fysiopraxis. 2019. Jaargang 28 december 2019/januari 2020.

Ummels D. Beekman E., Moser A., Braun S., Beurskens A. Ervaring van patiënten met een chronische aandoening met het gebruik van commercieel verkrijgbare activiteitenmeters in de fysiotherapie. Physios. 2020 Jaargang 12. Nummer 1.

\section{Oral presentations}

Ummels D, Daniëls N. Hoe een wearable werkbaar wordt. Ervaringsverslag na doorontwikkeling van wearables en apps. Lime meeting, Heerlen, 10 december 2019.

Ummels D, Ervaringen met het gebruik van commercieel verkrijgbare activiteitenmeters in de fysiotherapie van patiënten met een chronische aandoening: een kwalitatieve studie. Dag van de Fysiotherapeut, Den Bosch, 16 november 2019.

Ummels D, Patients experiences with commercially available activity trackers embedded in physiotherapy treatment: A qualitative study, Association for the Advancement of Assistive Technology in Europe congres, Bologna (Italië), 28 augustus 2019.

Ummels D, Koppert M. Activiteitenmeters en andere wearables in de fysiotherapie: wat en hoe? Fysio-Xperience, Eindhoven, 14 juni 2019.

Ummels D, Beekman E. The validity of activity trackers during activities of daily living. Dag van de Fysiotherapeut, Den Bosch, 8 december 2018.

Ummels D. Samen slimmer meten voor een betere gezondheid en zorg. Parkstad Zorgbeurs. Kerkrade, 3 november 2018.

Crijns F, Hochstenbach L, Ummels D. Hoe breng je de meetwetenschap naar de praktijk. Lime Meeting. Geleen, 11 oktober 2018.

Ummels D. 'De AcTiV LiFe studie. Commercieel verkrijgbare activiteitenmeters in de fysiotherapie en ergotherapie' Symposium activiteitenmeters in de zorg, Heerlen, 9 mei 2017. 
Ummels D. 'De AcTiV LiFe studie. Commercieel verkrijgbare activiteitenmeter in de fysiotherapie" Dag van de Fysiotherapeut, Utrecht, 11 november 2016.

\section{Poster presentations}

Ummels D., Beekman E., Moser A., Braun, S., Beurskens S. Patients experiences with commercially available activity trackers embedded in physiotherapy treatment: A qualitative study. Eindsymposium i2-CoRT, Hoensbroek, 20 april 2021.

Ummels D., Beekman E., Moser A., Braun, S., Beurskens S. Ervaringen met het gebruik van commercieel verkrijgbare activiteitenmeters in de fysiotherapie van patiënten met een chronische aandoening: een kwalitatieve studie. Dag van de Fysiotherapeut, Den Bosch, 16 november 2019.

Ummels D., Beekman E., Moser A., Braun, S., Beurskens S. Patients experiences with commercially available activity trackers embedded in physiotherapy treatment: A qualitative study. Caphri Research Day, Maastricht, Nederland, 6 november 2018.

Ummels D., Beekman E., Theunissen K., Braun S., Beurskens S. Validiteit van negen commercieel verkrijgbare activiteitenmeters in chronisch zieken tijdens een activiteitenprotocol met algemeen dagelijkse levensverrichtingen. Onderzoek in beweging, Maastricht, 27 februari 2018.

Ummels D., Beekman E., Theunissen K., Braun S., Beurskens S. Validiteit van negen commercieel verkrijgbare activiteitenmeters in chronisch zieken tijdens een activiteitenprotocol met algemeen dagelijkse levensverrichtingen. Dag van de Fysiotherapeut, Barneveld, 24 november 2017.

Ummels D., Beekman E., Theunissen K., Braun S., Beurskens S. Validity of activity trackers in people with a chronic disease in a real-life activity protocol. Caphri Research Day, Maastricht, 9 november 2017.

Ummels D., Thijssen D., Rewald S., Beekman E. Aquacycling een dragende kracht. Onderzoek in beweging, Maastricht, 2015. 
